(W)

Check for

updates

Cite as

Nano-Micro Lett.

(2020) 12:167

Received: 21 May 2020

Accepted: 12 July 2020

Published online: 15 August 2020

(C) The Author(s) 2020

\section{Recent Progress, Challenges, and Prospects in Two-Dimensional Photo-Catalyst Materials and Environmental Remediation}

\author{
Karim Khan ${ }^{1,2}$, Ayesha Khan Tareen ${ }^{2,3}$, Muhammad Aslam ${ }^{2,4}$,
} Rizwan Ur Rehman Sagar ${ }^{5}$, Bin Zhang ${ }^{2}$, Weichun Huang ${ }^{2}$, Asif Mahmood ${ }^{6}$, Nasir Mahmood ${ }^{7}$, Kishwar Khan $^{8}$, Han Zhang ${ }^{2}$, Zhongyi Guo ${ }^{1 凶}$

Karim Khan, Ayesha Khan Tareen, and Muhammad Aslam have contributed equally to this work.

$\triangle$ Karim Khan, karim_khan_niazi@yahoo.com; Han Zhang, hzhang@szu.edu.cn; Zhongyi Guo, guozhongyi@hfut.edu.cn

1 School of Electrical Engineering and Intelligentization, Dongguan University of Technology (DGUT), Dongguan 523808, Guangdong, People's Republic of China

2 Institute of Microscale Optoelectronics, Collaborative Innovation Centre for Optoelectronic Science and Technology, Key Laboratory of Optoelectronic Devices and Systems of Ministry of Education and Guangdong Province, College of Physics and Optoelectronic Engineering, Shenzhen Key Laboratory of Micro-Nano Photonic Information Technology, Guangdong Laboratory of Artificial Intelligence and Digital Economy (SZ), Shenzhen University, Shenzhen 518060, People's Republic of China

3 College of Materials Science and Engineering, Shenzhen University, Shenzhen 518060, People's Republic of China

4 Government Degree College Paharpur, Gomel University, Dera Ismail Khan, K.P.K, Islamic Republic of Pakistan

5 School of Materials Science and Engineering, Jiangxi University of Science and Technology, Jiangxi 341000, People's Republic of China

6 School of Chemical and Bio-Molecular Engineering, The University of Sydney, Sydney, NSW 2006, Australia

7 School of Engineering, The Royal Melbourne Institute of Technology (RMIT) University, Melbourne, VIC, Australia

8 Research Laboratory of Electronics (RLE), Massachusetts Institute of Technology (MIT), Cambridge, MA, USA

\title{
HIGHLIGHTS
}

- Current progress in preparations, structures, and physicochemical properties of two-dimensional photo-catalyst materials and environmental remediation.

- Propose approaches of diverse of two-dimensional photo-catalyst materials-based nanoplatforms, optimization strategies to enhance activity, and their diverse applications.

- Current challenges and potential advancement of the emerging of two-dimensional photo-catalyst materials. 
ABSTRACT The successful photo-catalyst library gives significant information on feature that affects photo-catalytic performance and proposes new materials. Competency is considerably significant to form multi-functional photo-catalysts with flexible characteristics. Since recently, two-dimensional materials (2DMs) gained much attention from researchers, due to their unique thickness-dependent uses, mainly for photo-catalytic, outstanding chemical and physical properties. Photo-catalytic water splitting and hydrogen $\left(\mathrm{H}_{2}\right)$ evolution by plentiful compounds as electron $\left(\mathrm{e}^{-}\right)$donors is estimated to participate in constructing clean method for solar $\mathrm{H}_{2}$-formation. Heterogeneous photocatalysis received much research attention caused by their applications to tackle numerous energy and environmental issues. This broad review explains progress regarding 2DMs, significance in structure, and catalytic results. We will discuss in detail current progresses of approaches for adjusting 2DMs-based photo-catalysts to assess their photo-activity including doping, hetero-structure

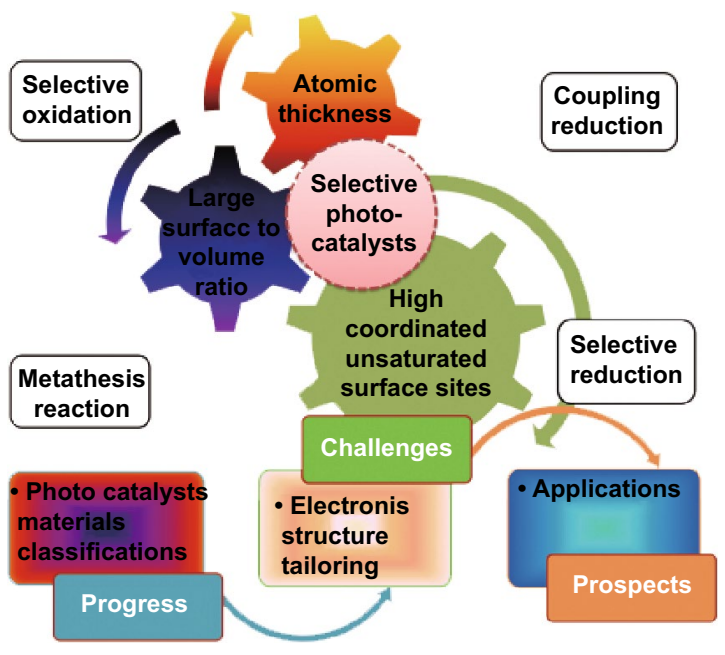
scheme, and functional formation assembly. Suggested plans, e.g., doping and sensitization of semiconducting 2DMs, increasing electrical conductance, improving catalytic active sites, strengthening interface coupling in semiconductors (SCs) 2DMs, forming nano-structures, building multi-junction nano-composites, increasing photo-stability of SCs, and using combined results of adapted approaches, are summed up. Hence, to further improve 2DMs photo-catalyst properties, hetero-structure design-based 2DMs' photo-catalyst basic mechanism is also reviewed.

KEYWORDS Two-dimensional materials; Photo-catalysts; $\mathrm{H}_{2} \mathrm{O}_{2} / \mathrm{H}_{2}$-production; Pollutant degradation; $\mathrm{CO}_{2}$ reduction

\section{Introduction}

The sustainable energy and chemical supplies demands are very essential for modern society that is necessary for our transportation, prosperity, and daily simplicity. The world's almost $85 \%$ energy demands are fulfilled by fossil fuelsbased energy production. Therefore, an increasing progress in modern society for pollution-free energy production gains attention of the researchers in all fields. In the past, larger part of worldwide energy formation was based on fossil fuel, which increases environment pollution and hence causes global warming [1-14]. In this modern society, development in different industries causes rapid population growth, which is further estimated to increase by two factors: the current energy required by 2050 to run industries around globe and their household uses [2-4, 15-26]. Presently, world's energy supplies are mainly reliant on fossil fuels, for example, coal, petroleum, and natural gases, which are quickly being spent. Utilization of fossil fuels will certainly cause particular gases' emission which are very injurious to the environment. Consequently, innovative findings in science and engineering are proceeded to address the barriers for efficient energy formation and environmental safety. Hence, production of the sustainable/renewable energy is a solution to meet up the rising worldwide energy demand and especially to solve environmental pollution issues [2-4, 27]. Conversely, fossil fuel-based energy is also widely used in chemical production on industrial level via inorganic/organic transformations by applying high-temperature/pressure circumstances. Although fossil fuel supplies are possibly sufficient for some next generations, durable cost of fossil fuels is undesirable because of un-sustainability of fossil fuels recognized as partial assets and rising greenhouse gases certified to enormous release of $\mathrm{CO}_{2}$-like hazard gases. The technical challenges to increase an industrially talented chemical process to protect a clean, renewable energy and to reduce harmful ecological impact are connected with the use of fossil fuels $[2,3,19,22]$. In this regard, renewable sustainable energy production is one of the significant solutions, especially hydrogen $\left(\mathrm{H}_{2}\right)$-based energy creation by photo-catalysts as well as electrocatalysts [2-4, 18, 19, 21, 25]. Here, we will mainly concern on photo-catalysts. The $\mathrm{H}_{2}$ has the maximum energy contented per weight in combustion fuels and manufactures simply water $\left(\mathrm{H}_{2} \mathrm{O}\right)$ as by-product $[3,4,19]$. Thus 
far, straightforward transfer of solar energy to fuel energy $\left(\mathrm{H}_{2}\right)$ and chemical energy was viewed as one of the green renewable ways to deal with energy and environmental pollution issues in the future [2-4, 17, 18, 21, 23-25]. Hence, $\mathrm{H}_{2}$ is considered as an ultra-clean, powerful, environment friendly, and hopeful another choice for meeting the future fuel necessities with environmental safety by less release of greenhouse gasses [2-4, 17-19, 21, 23, 24].

\section{Basic Properties of Photo-catalysis}

\subsection{Merits of Photo-catalysis}

The considerable reliance of worldwide economy on nonrenewable and geopolitical susceptible fossil fuel energies has led to necessity in advance technologies to protect alternative clean and renewable energy supplies. In between different renewable energy sources (i.e., wind, tidal, hydroelectric, ocean currents, biomass, geothermal, and solar), solar energy is by far the most abundant, low cost, pollution free, and sustainable. Even though the total solar energy the earth receives for one hour is greater compared to annual global energy expenditure, the most serious challenge remains collection and storage of this very diffuse form of energy to facilitate real-world application and non-interrupted fuel supply. Photo-catalysis can be basically explained as a method wherein photo-generated electrons $\left(\mathrm{e}^{-} \mathrm{s}\right)$ and holes $\left(\mathrm{h}^{+} \mathrm{s}\right)$ induce targeted redox reactions on light absorbers and/or co-catalysts loaded on it. A range of other invented renewable energy schemes, semiconductor (SC)-based photo-catalysis, in which infinite and clean solar energy can be acquired as a possible technology [28] achieved great interdisciplinary concentration for their various probabilities in energy and environmental uses. Efficient transformation of solar energy to solar fuel using photo-catalytic method was measured as very eventual enduring maneuver to resolve global energy and environmental concerns [29]. Naturally abundant sunlight and $\mathrm{H}_{2} \mathrm{O}$ splitting-based production of $\mathrm{H}_{2}$ by using sunlight were verified as regenerative, environment friendly, and vast techniques to resolve energy disaster and environmental pollution. In photo-catalysis method, a steady and capable photo-catalyst is an important aspect to attain a high efficiency of $\mathrm{H}_{2}$. For energy crises as well as environmental issues, SC-based photo-catalysis has enormous ability to guarantee long-lasting and sustainable development, because of direct consumption of green solar energy for formation of important $\mathrm{H}_{2}$ fuels and degradation of organic pollutants. Generally, four steps take place in the photo-catalytic process:

1. Light absorption

2. Creation of photo-generated $\left(\mathrm{e}^{-}-\mathrm{h}^{+}\right)$-pairs

3. Movement and recombination of photo-generated $\left(\mathrm{e}^{-}-\right.$ $\mathrm{h}^{+}$)

4. Redox reactions at photo-catalysts surface

How to understand it proficiently is very demanding, both kinetically and thermodynamically. The complexities lie in the subsequent features:

1. Maximum yielding of solar energy (mainly visible (vis) light) to produce enough energetic $\mathrm{e}^{-} \mathrm{s} / \mathrm{h}^{+} \mathrm{s}$

2. High mobility and long dispersion length of photo-generated $\mathrm{e}^{-} \mathrm{s} / \mathrm{h}^{+} \mathrm{s}$ to suppress bulk recombination

3. Sufficiently strong reduction power of photo-generated $\mathrm{e}^{-} \mathrm{s}$ and $\mathrm{h}^{+} \mathrm{s}$ to persuade reactions, specially $\mathrm{H}_{2} \mathrm{O}$ oxidation that demands four $\mathrm{e}^{-} \mathrm{s}$

4. Plentiful surface locations for forward target reactions as an alternative of back reactions (e.g., $\mathrm{H}_{2}$ and $\mathrm{O}_{2}$ reaction to fabricate $\mathrm{H}_{2} \mathrm{O}$ )

Such four subjects represent a significant research pathway. Moreover, three steps span a huge timescale from $10^{-15}$ to $10^{-1} \mathrm{~s}$. What's more demanding are intrinsic conflicts between necessities for three key steps. Minimum three factors are considered here:

1. Increasing light absorption range (reduced bandgaps) generally leads to small reduction capability of photogenerated e-s and/or a lower oxidation capability of photo-generated $\mathrm{h}^{+} \mathrm{s}$;

2. The very low mobility of $\mathrm{h}^{+} \mathrm{s}$ compared to e-s in most SCs does not support rate-determining $\mathrm{H}_{2} \mathrm{O}$ oxidation reactions,

3. The difference in random distributions of oxidation and reduction reaction sites and required migration of $\mathrm{e}^{-} \mathrm{s} /$ $\mathrm{h}^{+} \mathrm{s}$ in diverse directions.

The strong underlying conflicts connected with photophysical process, electronic properties, and catalysis principles build recognition of highly efficient photo-catalysis as a very challenging process. To solve these challenges, it is significantly important to accurately control every fundamental step depending on a comprehensive consideration of 
photo-catalysis and structure property interactions. Therefore, first we are going to explain SC materials' suitability for photo-catalytic nature.

\subsection{General Selection of SC Photo-catalytic Materials}

Generally, the photo-catalysis is an accelerated photoreaction method in existence of SC photo-catalyst, in which photons with energy $h v \geq E_{\mathrm{g}}$ ( $E_{\mathrm{g}}=$ band gap (BG) energy) of photo-catalyst are adsorbed to photoexcite free electrons $\left(\mathrm{e}^{-} \mathrm{s}\right)$ to conduction band $(\mathrm{CB})$, creating holes $\left(\mathrm{h}^{+} \mathrm{s}\right)$ in valence band (VB). Photo-generated $\left(\mathrm{e}^{-}-\mathrm{h}^{+}\right)$-pairs participate in an important part for solar energy transfer method, for example, solar $\mathrm{H}_{2} \mathrm{O}$ splitting, $\mathrm{CO}_{2}$ reduction, and photocatalytic pollutant degradation. Although photo-generated carriers in excited states are less stable, they recombine easily, which results in low conversion effectiveness of photocatalysis [30]. By the way, since discovery of photo-catalytic $\mathrm{H}_{2} \mathrm{O}$ splitting with $\mathrm{TiO}_{2}$ in 1972, great effort was applied in progress of photo-catalysts for an efficient photo-catalytic method [31]. The SC-based photo-catalysis concerned huge research attention, [32] since it was considered very novel solution to manage energy deficiency and environmental pollution problems [33]. The sunlight as an exterior driving force can split $\mathrm{H}_{2} \mathrm{O}$ into $\mathrm{H}_{2}$ and $\mathrm{O}_{2}$, reduce $\mathrm{CO}_{2}$ to chemical and valuable fuel, as well as terminate pollutants entirely [34]. Normally, the main significant efforts in the photocatalytic development are categorized as light absorption, charge separation, transfer, and surface redox responses. By irradiation of photo-catalysts, it absorbed sunlight, which excites to produce $\left(\mathrm{e}^{-}-\mathrm{h}^{+}\right)$-pairs, when $h v \geq E_{\mathrm{g}}$, leaving e-s in $\mathrm{CB}$ and $\mathrm{h}^{+} \mathrm{s}$ in $\mathrm{VB}$, respectively. After that, photo-generated $\mathrm{e}^{-} \mathrm{s}$ and $\mathrm{h}^{+} \mathrm{s}$ are diffused to material surface and also transferred to surface active sites, prior to connection with surface reactions. Sometimes, charge carrier's recombination occurs and crystal structure, particle size, crystallinity, surface morphology, etc., strongly influenced separation efficiency. At last, target molecules are adsorbed on the surface of materials and experience charge addition development and desorption to make final results [35].

In between $\left(\mathrm{e}^{-}-\mathrm{h}^{+}\right)$-recombination process, unnecessary heat is created, which causes a negative role in photo-catalytic production. In the photo-catalysis method, a stable and efficient photo-catalyst is an imperative feature to get high efficiency of $\mathrm{H}_{2}$. Additionally, the driving force of solar light photo-catalysis demands suitable SCs to perform various photo-catalytic responses, for example, $\mathrm{H}_{2} \mathrm{O}$ splitting to manufacture $\mathrm{O}_{2}$ and $\mathrm{H}_{2}, \mathrm{CO}_{2}$ reduction to hydrocarbon fuels, degradation of organic pollutants, disinfection of bacteria, and selective formation of organic compounds [36]. The milestone occurrence of photo-catalytic $\mathrm{H}_{2} \mathrm{O}$ splitting, by $\mathrm{TiO}_{2}$-based electrodes in an ultraviolet (UV) light, was started from revolutionary research co-authored by Fujishima and Honda [37]. In 1976, photo-catalytic degradation of organic contaminants was studied by Carey et al. [38] using $\mathrm{TiO}_{2}$ in aqueous suspension. In 1979, Inoue and co-authors examined photo-catalytic reduction of $\mathrm{CO}_{2}$ to a range of organic compounds by $\mathrm{SC}$ materials, for example, $\mathrm{TiO}_{2}, \mathrm{SiC}, \mathrm{ZnO}, \mathrm{CdS}$, and $\mathrm{GaP}$, in aqueous solution. After that, various considerable progresses were made in the formation of very proficient SC-based photo-catalysts. Up to now, several SC photo-catalysts were exploited and utilized in $\mathrm{H}_{2} \mathrm{O}$ splitting. Based on composition, photo-catalysts are usually classified into three kinds:

1. Metal oxides (MOs)

2. Metal chalcogenides

3. Metal-free photo-catalysts

So far, hundreds of SC materials are discovered for different photo-catalytic uses by tuning a range from composition, electronic, and crystal structure. While important accomplishment was achieved in optimizing photo-catalytic performance, most photo-catalysts still suffer from relatively low photo-catalytic efficiencies that are much lower compared to the necessities for probable realistic uses. Based on previous research investigation, probable UV-Vis-active and vis-light-active photo-catalysts included $\mathrm{TiO}_{2}, \mathrm{ZnO}, \mathrm{Fe}_{2} \mathrm{O}_{3}, \mathrm{CdS}, \mathrm{Bi}_{2} \mathrm{WO}_{6}, \mathrm{BiVO}_{4}, \mathrm{Ta}_{2} \mathrm{O}_{5}$, $\mathrm{Ta}_{3} \mathrm{~N}_{5}, \mathrm{TaON}, \mathrm{C}_{3} \mathrm{~N}_{4}$, and so on [39]. To date, emerging high-quality SC photo-catalyst for surmount recovery of energy deficiency and environmental hazards is a great research field [36]. Despite quick progress of conventional photo-catalysts, they are still facing numerous major challenges:

1. Many SCs, particularly MOs, can absorb UV light because of their wide BG [40]

2. A few SCs are not appropriate for entire $\mathrm{H}_{2} \mathrm{O}$ splitting, due to their inappropriate band location and because they only show either $\mathrm{H}_{2} \mathrm{O}$ oxidation or reduction activity [41] 
3. In relocation of photo-generated charge carriers to surface reactive sites, charge recombination happens simply for bulk and on photo-catalysts' surface [42]

4. The majority of bulk SC reaction active sites cannot be exposed to surface and are utilized in the photo-catalytic process [29]

Therefore, key issues to attain an excellent photocatalytic performance depend on normal mean of highefficiency photo-catalysts. In recently discovered new potential photo-active materials, 2DMs got much consideration. Bearing in mind various characteristics and advantages, the promising 2DMs with suitable energy band configuration can stimulate new visions [14, 43, 44]. Recent research in 2DMs has advanced the modernized attention in $\mathrm{p}-\mathrm{n}$ junction; the oldest electrical mechanism was employed in electronics and optoelectronics devices research. The 2DMs offer an amazing flexibility to propose a novel (p-n)-junction device configuration, not workable through usual 3D bulk SCs. The 2DMs signify a promising category of materials that have NSs-like configuration with thickness of just one or few atoms [45]. Attempts were ignited through innovation of graphene $(\mathrm{G})$ in 2004, a single-layer (SL) carbon material along outstanding thermal, mechanical, and electrical characteristics [46]. Ever since, a range of G-like 2D photo-catalysts were become a relevant topic in photo-catalysis field. The 2D photo-catalysts showed special chemical and physical properties in contrast to their bulk counterparts. Emerging 2DMs with unique structural and electronic properties and appropriate band structure have showed huge potential of achieving the desired photo-catalytic efficiency. There are numerous features which influence the photocatalytic efficiency of photo-catalysts, e.g., composition, BG, crystallinity, surface state, and morphology of SC materials, and interfacial properties of components for composite photo-catalysts.

In view of necessities for competent light adsorption and photo-generated carrier separation and transport, if possible, a photo-catalyst must contain an elevated specific surface area (SSA), good crystalline structure, stability, and an appropriate band structure [29]. The 2DMs arrangement can supply huge SSA and a large fraction of low coordinated surface atoms to produce further UV light, whereas photon absorption in bulk materials or nanoparticles (NPs) is frequently inadequate through transmittance of light and reflection at grain boundaries [47]. Furthermore, as a result atomic size thickness significantly decreases the migration distance; charge carriers produced in 2DMs interior will be quicker to transfer on surface compared to bulk materials (Fig. 1a). It will significantly decrease recombination chance of photo-generated carriers and support photo-catalytic method. Finally, regarding surface redox reactions, distinctive 2DMs configuration along elevated ratio of surface atoms to whole atoms can cause new SAS to speed up the reaction development. Additionally, atomic breakdown energy develops into comparatively minute when thickness decreased to atomic level and so additional surface defects will come into view. These surface defects will promote and improve target molecule adsorption to make strong interaction, easy charge transfer, and better activation procedure. Photo-catalysts with such characteristics and 2DMs configuration receive high interest, and a great number of related studies were performed [32].

To concentrate on these challenges, formations of new and more competent photo-catalysts are required to energetically investigate in this field [49]. The 2D structures along with foreign electronic properties and a high SSA are formed from layered [50] and non-layered [48] materials. The layered materials are identified through strong in-plane bonds and weak van der Waals (vdWs) force in layers. On the other hand, Ajayan and co-workers [48] recently discovered an efficient formation approach via the progressive conversion of non-vdW solids to $2 \mathrm{D}$ vdWs transition metal chalcogenide (TMDCs) layers with recognized $2 \mathrm{H}$ (trigonal prismatic)/1T (octahedral) segments (Fig. 1b, c). Conversions, obtained after exposing non-vdWs solids to chalcogen vapors, were controlled utilizing enthalpies and reaction products vapor pressures. Heteroatoms-substituted (e.g., phosphorus and yttrium) TMDCs were also formed by the same scheme, so a general formation scheme is allowed to form phase-selected TMDCs' 2D configurations with excellent stability at elevated temperatures (about $1373 \mathrm{~K}$ ) and obtain scalable manufacture of SLs. These 2D TMDCs have wide uses in catalysis, electronics, and energy storage applications. On account of remarkable structure-based, chemical and physical, properties of $2 \mathrm{DMs}$, the construction of few-layer (FL) or single-layer (SL) 2DMs provokes broad attention as talented photo-catalysts with numerous benefits: 

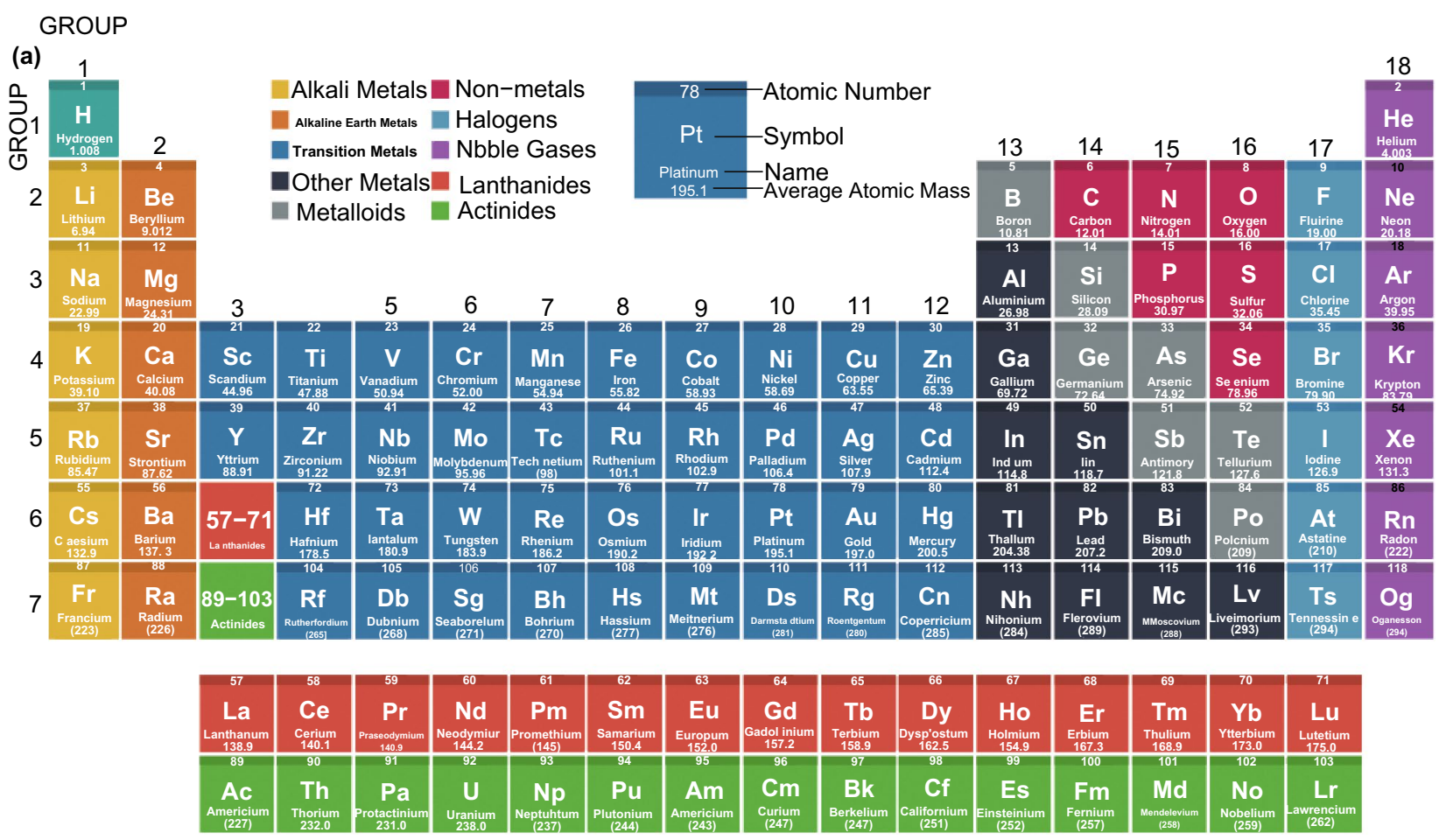

(b)

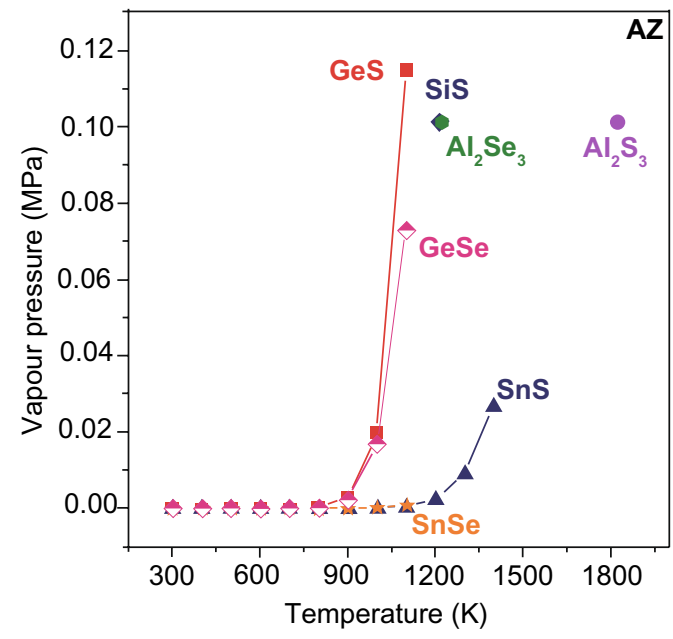

(c)

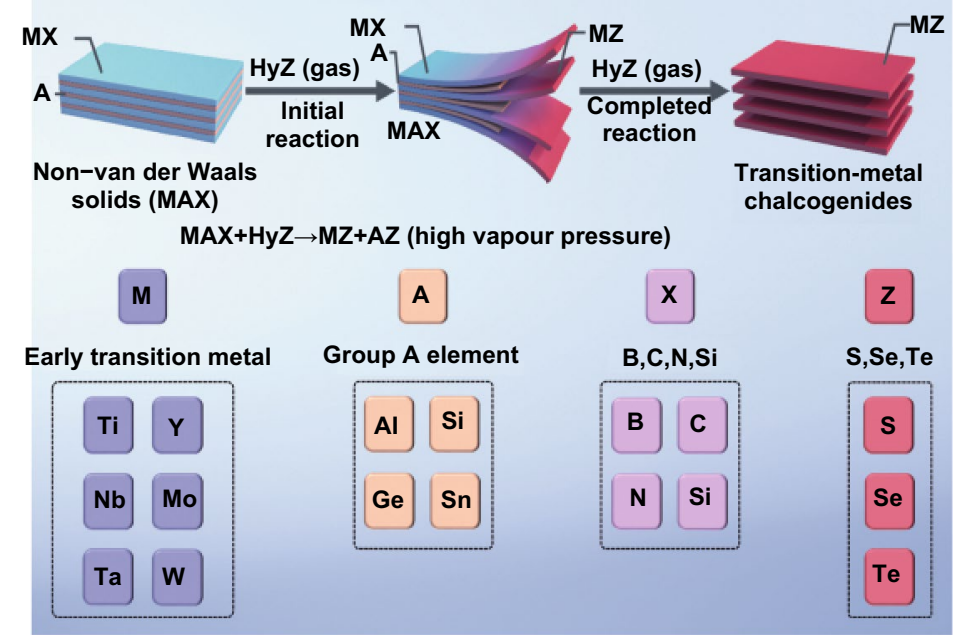

Fig. 1 a Periodic table shows discovered 2DMs. b Temperature and vapor pressure relations for a variety of AZ substances. c Scheme for conversion of non-vdWs solids to 2D vdWs TMDCs, where non-vdWs solids like MAX phases are gradually transferred to 2D TMDCs through a topological conversion response (MAX $+\mathrm{HyZ}$ (gas) $\rightarrow \mathrm{AZ}+\mathrm{MZ}$ ), related to volatile AZ products. Adapted with permission from Ref. [48]

1. The BG and light absorption of 2D-SC can adjust via altering layers number [51].

2. The $\left(\mathrm{e}^{-}-\mathrm{h}^{+}\right)$-recombination in case of bulk can decrease because of atomic size of 2DMs [52].

3. The SSA of the SCs is significantly enhanced, and most of the SASs can be exposed at surface and included in photo-catalytic reaction [47].
Along with the different 2D photo-catalysts with only FLs or SL structure, G-based photo-catalysts, 2D oxides, $2 \mathrm{D}$ chalcogenides, $2 \mathrm{D}$ graphitic carbon nitride $\left(\mathrm{g}_{-} \mathrm{C}_{3} \mathrm{~N}_{4}\right)$, and other 2D-SCs started gaining huge attention in photo-catalysis [49]. Although 2D photo-catalysts are viewed as talented materials to exchange solar energy into chemical energy as 
$\mathrm{H}_{2}$-formation, there are a number of hurdles, which limit their uses [49], as follows:

1. The exciton binding energy in 2DMs-based photo-catalysts was significantly enhanced because of smaller unfavorable $\mathrm{e}^{-}$-screening than bulk material [53].

2. Some 2DMs-based SCs are not stable in aqueous solution or air; thin-layer 2D SC can be assembled collectively or oxidized through photo-generated $\mathrm{h}^{+} \mathrm{s}$ during reaction, which leads to deterioration in photo-catalytic activity [54].

3. While $\left(\mathrm{e}^{-}-\mathrm{h}^{+}\right)$-pairs recombination is less compared to bulk SCs, it still resides in 2D photo-catalysts [55].

4. Reduction potential and oxidation potential of few 2D-SCs are not enough in overall $\mathrm{H}_{2} \mathrm{O}$ splitting [55].

To address such issues, a range of approaches were designed to increase photo-catalytic activity of 2DMsbased photo-catalysts, for example, doping with a metal or nonmetal elements, inducing defects, and coupling with metal or SCs, which will be discussed in detail in the upcoming sections [56]. In fact, photo-activity of photo-catalysts relies on their properties, for instance, electron affinity, crystal structure, BG, and interface in photo-catalyst as well as co-catalyst [57]. Consequently, for well-organized transfer of $\mathrm{H}_{2} \mathrm{O}$ to $\mathrm{H}_{2}$, the mixing of photo-catalyst and co-catalyst required novel interface structure. This kind of interface can optimize absorption of light for photo-catalysts and support $\mathrm{e}^{-} / \mathrm{h}^{+}$separation. Normally, bigger contact area at interface can offer enough charge transfer and trapping channels for parting $\left(\mathrm{e}^{-}-\mathrm{h}^{+}\right)$-pairs generated by incident light [49]. The abovementioned problem has one another solution, which is the hetero-structure formation of 2DMs. In contrast to 0D-1D, 1D-1D, 0D-2D, and 1D-2D interfaces, 2D-2D coupled hetero-structure-based interfaces concerned broad concentration in photo-catalysis due to their particular advantages, as follows [49]:

1. The creation of intimate interface in two SCs is in support of exciton dissociation, which enhances the photocatalytic quantum efficiency [58].

2. It is simplistic and proficient to structure the intimate interface in 2DMs-SCs, even if they have some mismatch of lattices [59].

3. Large lateral size along with high SSA leads to huge contact area in 2D/2D photo-catalysts that advance $\left(\mathrm{e}^{-}\right.$ $\mathrm{h}^{+}$)-pairs' separation and transfer [60].
4. The band potential coordinated to overall $\mathrm{H}_{2} \mathrm{O}$ splitting by integrating $\mathrm{H}_{2} / \mathrm{O}_{2}$-evolution photo-catalyst. Therefore, oxidation and reduction influence of SCs is balanced for $\mathrm{H}_{2} \mathrm{O}$ splitting [61].

5. Creation of $2 \mathrm{D} / 2 \mathrm{D}$ hetero-structure is advantageous to develop stability of photo-catalyst because of increase in photo-corrosion and agglomeration [62].

As a sustainable technology, the SC photo-catalysis has gained significant attention in the recent decades due to possible ease/resolve energy and environmental pollution concerns. Therefore, due to 2D/2D interface advantages, many 2D/2D structures are formed recently to improve photocatalytic performance of photo-catalysts [63, 64]. Based on these advantages, we are going to summarize most of the related topics, which can further improve the photo-catalytic phenomenon for $\mathrm{H}_{2} \mathrm{O}$ splitting $\left(\mathrm{H}_{2} \mathrm{O}\right.$ oxidation and $\mathrm{H}_{2}$ evolution), $\mathrm{CO}_{2}$ reduction, $\mathrm{N}_{2}$ fixation, organic production, removal of pollutants based on G-based photo-catalysts, 2D oxides, 2D-chalcogenides, $2 \mathrm{D} \mathrm{g}-\mathrm{C}_{3} \mathrm{~N}_{4}$, and some other 2D-SCs.

The 2DMs reviewed here are considered as low-dimensional materials with thickness ranging from SL to few nanometers $(\mathrm{nm})$ by means of basal plane controlling total surface area, and 2DMS-based SCs photo-catalysis principles, synthesis, and stability will be briefly reviewed. Here, up-to-date development of 2DMs-based photo-catalysts is summarized, and significant evaluations of categorizing and convenient production method of 2DMs-based photocatalysts are presented. To further boost these results, different policies to engineer electronic structure of 2DMs-based photo-catalysts are summed up, such as component tuning, thickness tuning, defect, and doping engineering. Hybridization with insertion of outside components and keeping 2D structure is explained to improve photo-catalytic efficiency, for example, quantum dots (QDs)/2DMs, single atoms/2DMs, molecular/2DMs, and 2D-2D stacking materials. Therefore, we will give a concise explanation of recently developed 2DMs, their applications in photo-catalysis, and the promising approaches for the photo-activity progress from the perspective of chemical doping, hetero-structure layout as well as functional structural design assembly. More importantly, attention will be paid to advancement of versatile photo-catalytic applications of 2DMs-based photocatalysts in $\mathrm{H}_{2} \mathrm{O}$ oxidation, $\mathrm{H}_{2}$ evolution, $\mathrm{CO}_{2}$ reduction, $\mathrm{N}_{2}$ fixation, organic synthesis, and elimination of pollutants. 
Besides, manufacture approaches and characterization methods of 2D/2D photo-catalysts are also reviewed. Finally, ongoing opportunities and challenges for upcoming progress of 2DMs-based photo-catalysts in this exhilarating yet still upcoming area of research will be projected [29, $32,49]$ and a short summary of present research position and challenges, with respect to 2DM-based photo-catalysts for photo-catalysis applications, will also be explained [29]. It is extremely important and insistent to present a timely updated widespread review on this matter to endorse further progress in the upcoming direction [32].

\subsection{Benchmark Photo-catalysts}

As it appears that too long step is in our conviction also a little that considered in future investigations that are association derived from present price of photo-catalytic materials. This feature looks alienated to laboratory-level work, deals with elementary information, and consequently accords with the rule not to be hampered by funds restrictions. On the other hand, we must admit that exploration is increasingly related to industry, and funding is governed through financial analysis of project, so this feature can no longer be ignored. In recent publications, it is expected to go forward in claims on importance of reported materials due to the absence of precious metals that were still considered as co-catalysts [65]. It is a too common assumption that does not inform the features of cheaper material; consequently, not including a precious metal (frequently applies in small quantity) is not essentially an economic choice, because material still depends on expensive starting materials, or tiresome reaction conditions (purification, temperature, solvent, etc.) that finally end up in inflating the cost of the proposed catalyst. It may take time to endeavor a little quantitative cost estimation to propose photo-catalyst, not only because the time is a necessary feature, but also getting support for optimizations in this field from other researchers [66]. Coming to the point, the following three key components are proposed to be explored to compare the built-in photonic effectiveness of a variety of photo-catalyst materials in laboratories.

1. Incident photon flux (photon numbers with respect to wavelength per time).

2. "Optimum rate" (achieve the highest photo-catalytic rate through changing photo-catalyst quantity in a specified reactor).
3. Rate of reactant expenditure or product evolution (at optimum rate under diverse reaction conditions).

The increase in heterogeneous photo-catalysis interest and other solar fuel conversion schemes will unavoidably lead to more research in this area. Unfortunately, many research works enclose imprecision while studying photo-catalytic measurements, particularly while reporting gas evolution [67]. It is taken toward benchmark materials' selection difficulties as there is non-reliability in efficiency measurements. In the literature, some common errors are observed in expectancy of increasing overall quality this direction. It is recommended that classification for exploring photocatalytic rate is given as follows:

1. Reactant conversion kinetic or product formation rates.

2. Incident photon flux with respect to wavelength.

3. Activities or partial pressures of reactants and sacrificial reagents.

4. Solution type, electrolyte concentration, and $\mathrm{pH}$.

5. Quantity of photo-catalyst, co-catalyst, and solution.

6. Flow rate of reactor and volume/dimension of reactor.

Following combined experiments carried out to assess photo-catalytic performance in likely comprehensive approach, the next step is to comprehend how recently studied photo-catalyst ranks in between present photo-catalysts. Benchmark cannot recommend from all considerations mentioned previously. A lot of investigations have been inadequately performed on this significant part of work, and only contrast materials are benchmark reference catalysts, for instance $\mathrm{TiO}_{2}$ Degussa P25. It certainly offers an early essence of photo-catalysts, but all the time comparison cannot provide a good judgment and is not enough to validate the published results. It might appear understandable, but arises a first theoretical question: Is model reported activity vs. Degussa P25 still possessed nowadays? We are bombarded with a variety of novel guidelines of editors, industries, grant agencies, and so on, that it is vital that upcoming photo-catalytic studies must concentrate on utilization of vis-light irradiation. It is a logical insight that provided strong relationship of photo-catalysis with sustainability, future realistic growth should take toward green energy, and process must hinge on utilization of sun light. A lot of energy is irradiated through solar spectrum in the range of vis spectrum (43\%), but still more is in fact irradiated through IR rays (52\%), yet at this moment it is complicated 
to utilize it for SC photo-catalysis. It is confined energy to produce requisite charge separation in the majority of SCsbased photo-catalysts. However, it is valuable to note that only some ground-breaking works on exploitation of IR radiation have come forward [68-71], that hold interesting promises for future research development. Indeed, UV region (5\%) is far too little, and so there is commonsense that wide BG $(\geq 3.0 \mathrm{eV}) \mathrm{SCs}$ by themselves can no longer participate in leading role and become outdated unless investigated for development of strategies built around multicomponent structural arrangements, for example Z-schemes and $\mathrm{p}-\mathrm{n}$ junctions. Therefore, it looks conflict that activity must be indefinitely benchmarked against a UV-active SC, i.e., Degussa P25.

In these days, state-of-the-art catalysts' table of comparisons are emerging more frequently in research work. These are more helpful, if selected with care. Tables should not evaluate one but maximum possible activity potential factors. A comparison between QY does not reveal a lot about catalyst selectivity and stability, which are the two equal significant conditions of comparison. The experiment duration choice for calculating QY is arbitrary and thus can be simply turned to researchers' expediency, losing objective, particularly while kinetics of product formation are not steady. Furthermore, a photo-catalyst with superior AQY or QY could be simply synthetically better, as exclude a donation to evolve product through other mechanisms working in dark. One more significant feature is that AQY experiments are generally performed with monochromatic light source, and as declared already, QY differs along excitation wavelengths. Tables of comparison completely conversed on QY preferably demand of comparison depend on polychromatic lights sources or as a minimum between photo-catalysts mainly absorbing in same narrow wavelengths range (a situation hard to attain). It is obvious to compare standard catalyst and synthetic catalysts discovered under similar catalytic conditions. Terminology is an additional feature not to be underestimated, as it can be the source of perplexity. As distinct earlier QY and AQY refer to quantity of consumed reactant (or product formation), other International Union of Pure and Applied Chemistry definitions more frequently utilized in heterogeneous photo-catalysis regarded as photonic efficiency (PE) and quantum efficiency (QE):

$\mathrm{QE}=$ photochemical events/absorbed photon flux

$\mathrm{PE}=$ photo-reaction rate/rate of incident photons [72]
Researchers need confirmation whether they are comparing the same factors. In general, we discourage a benchmark prepared completely for QY, $\mathrm{PE}$, or QE, as in our estimation it is imperfect and deceptive [73]. Other activity data can propose extra basic information: Reporting product formation rates over unlimited time offers evaluation of catalyst stability and not very precise suggestion of probable diverse system, as well as a comparison on such terms is necessary for designing catalysts to be formed at commercial level. Compared rates should be studied for catalyst per both surface area and mass, for cause elucidated above.

\section{Classification of 2DMs for Photo-catalysts}

Advancement in material and engineering science over the past years has allowed huge development in catalysis, sustainable energy production, sensor, and electronics. Novel spectroscopy and nano-fabrication techniques offered tools to comprehend primary materials' properties and to materialize their functionalities by adjusting their configuration and composition. It leads to enormous advancement in multi-component industrial catalysts [73] (e.g., become weak after treatment [74]), excellent chemical production, electrocatalysis (e.g., fuel cell catalysts [75]), and photocatalysis [76]. This growth was not only governed through turnover and market demands but also through elevating community understanding, rules for environmental safety, and sustainable growth. Currently rising sustainable development and technologies create utilization of a broad range of components, of which some are rare and unequally spread on earth and therefore have economic viability and at probable risk supply. In some way ironically, sustainability and risks associated with material are frequently ignored in academic-level investigation. It is due to functionality and performances during working conditions generally prevailed over synthesis and takes apart costs; hence, evaluation of material criticality and its viability is very intricate and basically goes ahead [77]. However, a basic point of view is materials' sustainability for final target in renewable energy synthesis. Certainly, as clean energy is almost limitless (e.g., solar, wind), materials and chemicals utilized to transfer it to real electrical energy are obtain rarely. Notably, precious metals group (i.e., platinum), rare-earth elements, gallium, aluminum cobalt, and many others [78] are indispensable components of immensely utilized commercial catalysts. If 
dependence can be decreased via replacement, such materials would be recycled more competently in the future to circumvent economic disturbances and increasing reserved competition [79]. These materials should have the following properties:

1. Catalyst durability upgrading through material design (post-modifications, confinement)

2. Lowering noble/rare metal loadings, whereas upholding high activity, through maximizing active surface area (atomic-level thickness in low-dimensional materials)

3. Substituting significant components with cost-efficient and abundantly accessible ones (base metals, carbocatalysis)

4. Enhancement of durability in catalysts' synthesis and removal (green chemistry-based catalyst recycle)

5. Evaluation of toxicity and environmental effect of catalyst materials

The catalysts' nano-structure is another supreme feature to be considered as it can be used to analytically study and compare diverse catalysts to realize tendency in activity. Generally, the size of photo-catalyst materials also affects their electronic arrangement originated through quantum confinement effects (less than $10 \mathrm{~nm}$ ) and degree of interface with support, as smaller sized photo-catalyst materials have a larger portion of atoms at metal support edge [57]. For example, Taejong Paik et al. [80] defined the optical BG increased in tungsten oxide $\left(\mathrm{WO}_{x}\right) \mathrm{NWs}$ compared to stoichiometric $\mathrm{WO}_{3}$ bulk counterpart, because of Burstein-Moss shift. This increment confirmed direct photo-catalytic $\mathrm{H}_{2}$ evolution from $\mathrm{WO}_{x} \mathrm{NWs}$ via alcohol photo-reform. The stable $\mathrm{H}_{2}$ production on platinized $\mathrm{WO}_{x} \mathrm{NWs}$ is pragmatic under conditions where platinized bulk $\mathrm{WO}_{3}$ and bulk $\mathrm{WO}_{2.9}$ powders either do not show activity or show very low rates, proposing that enhanced surface area is the answer for enhanced activity. As a result, controlled size and composition can cause unanticipated and important alterations in SC photocatalytic materials properties [81]. As an ideal candidate for photo-catalysis, the mainly studied 2DMs-based photocatalysts can be divided into different types: counting, MOs, metal composite oxides, MHOs, bismuth-based materials, metal chalcogenides, and metal-free photo-catalysts. Based on photo-catalyst compositions, the 2DMs used in photocatalysts can be mainly categorized as illustrated in Fig. 2. The 2DMs can be synthesized either through exfoliation of

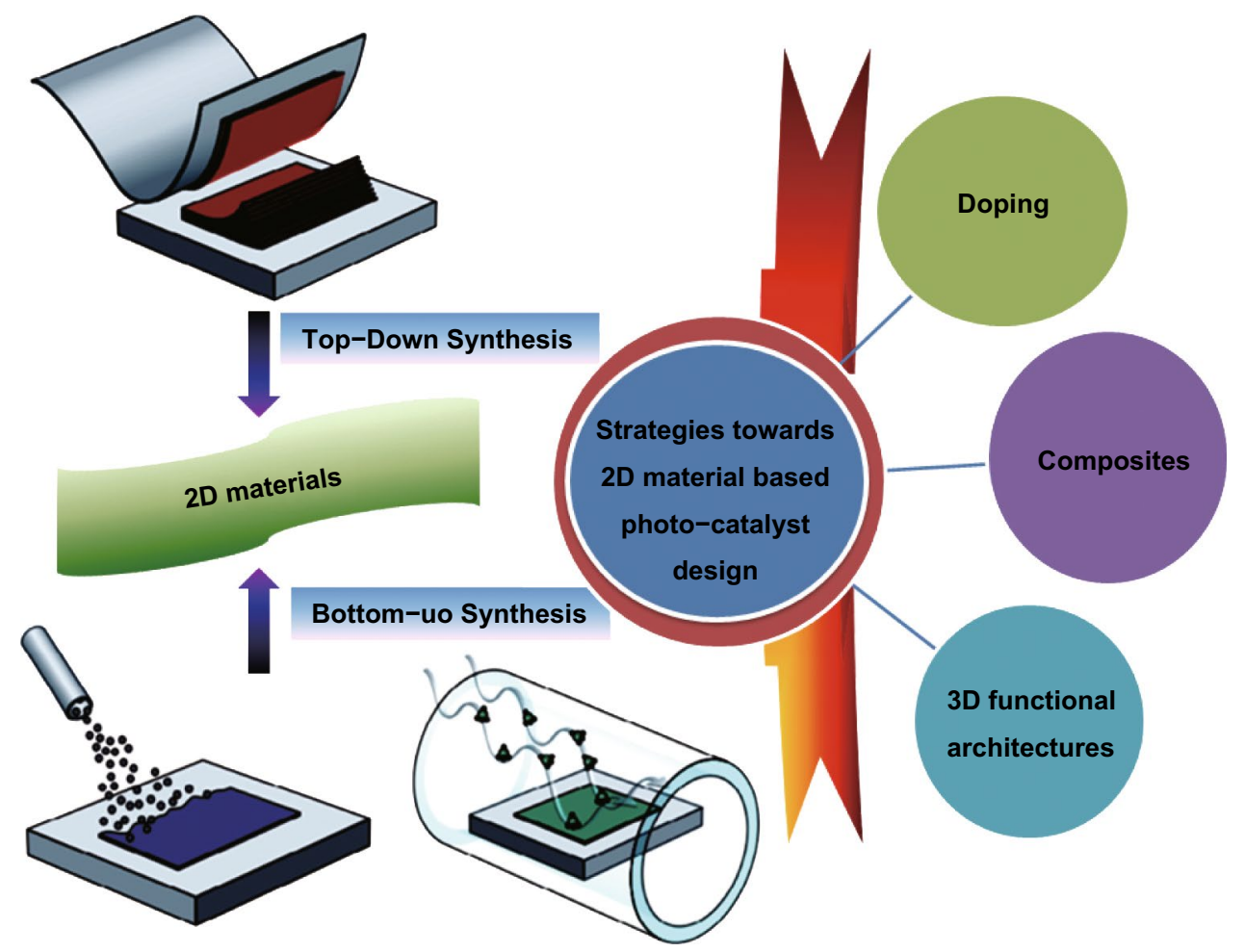

Fig. 2 Scheme of 2DMs synthesis and strategies toward 2DM-based photo-catalyst design 
parent layer material through top-down method or formed from small molecules using bottom-up self-assembly technique. Synthesis of 2DMs with tunable layer number, edge morphology, and degree of crystallinity is vital for utilizing these materials for elevated activity catalytic applications and is also discussed in our recently published reviews $[3,4,19]$. Therefore, in this part, we will only provide a concise introduction for basic properties with small explanations about the synthesis strategies of such three types of 2DMs, which are utilized for photo-catalysis applications.

\subsection{D metal Oxides (MOs) NSs}

The MOs are broadly studied for photo-catalysts. Certainly, fabricating 2DMs-based MOs is considered as an efficient way to maximize SSA and charge migration and hence gets a competent photo-catalytic performance [32]. So far, numerous MOs with 2DMs structures have been formed and used in photo-catalysis applications, for example $\mathrm{TiO}_{2}, \mathrm{Fe}_{2} \mathrm{O}_{3}$, $\mathrm{Cu}_{2} \mathrm{O}, \mathrm{ZnO}, \mathrm{WO}_{3}, \mathrm{SnO}, \mathrm{In}_{2} \mathrm{O}_{3}, \mathrm{CeO}_{2}, \mathrm{HNb}_{3} \mathrm{O}_{8}$, etc. [82]. Due to the basic non-layered structure feature, some 2D-MOs were complex to be formed by facile ultrasonic exfoliation technique from their bulk counterparts. So, numerous other means were applied for controlled formation of 2D-MOs. For example, a lamellar inorganic-organic hybrid intermediate policy was planned to form ultra-thin $\mathrm{TiO}_{2} \mathrm{NSs}$ [82]. Utilizing Ti-isopropoxide as a Ti source, octylamine as a capping reagent, 2-phenyl ethanol as solvent, lamellar $\mathrm{TiO}_{2}$-octylamine hybrid precursors were obtained via solvothermal process (Fig. 3) [32]. The ultrasound-based exfoliation-resulted powder was washed to eliminate octylamine and get clean, ultra-thin $\mathrm{TiO}_{2}$ NSs. The AFM result showed (a)

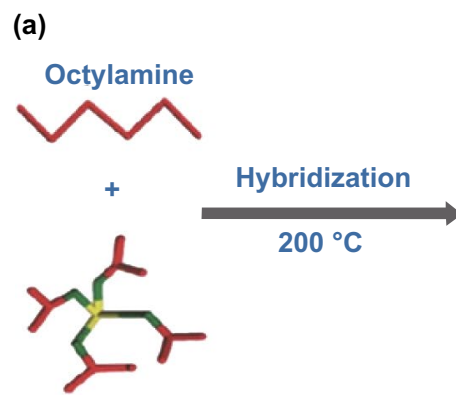

Ti-isopropoxide

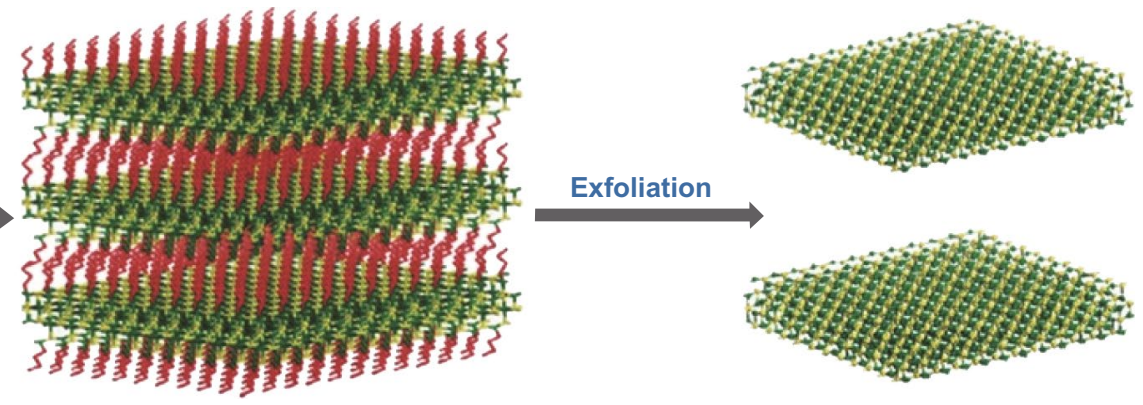

Lamellar $\mathrm{TiO}_{2}$-octylamine hybrid
Ultrathin $\mathrm{TiO}_{2}$ flakes
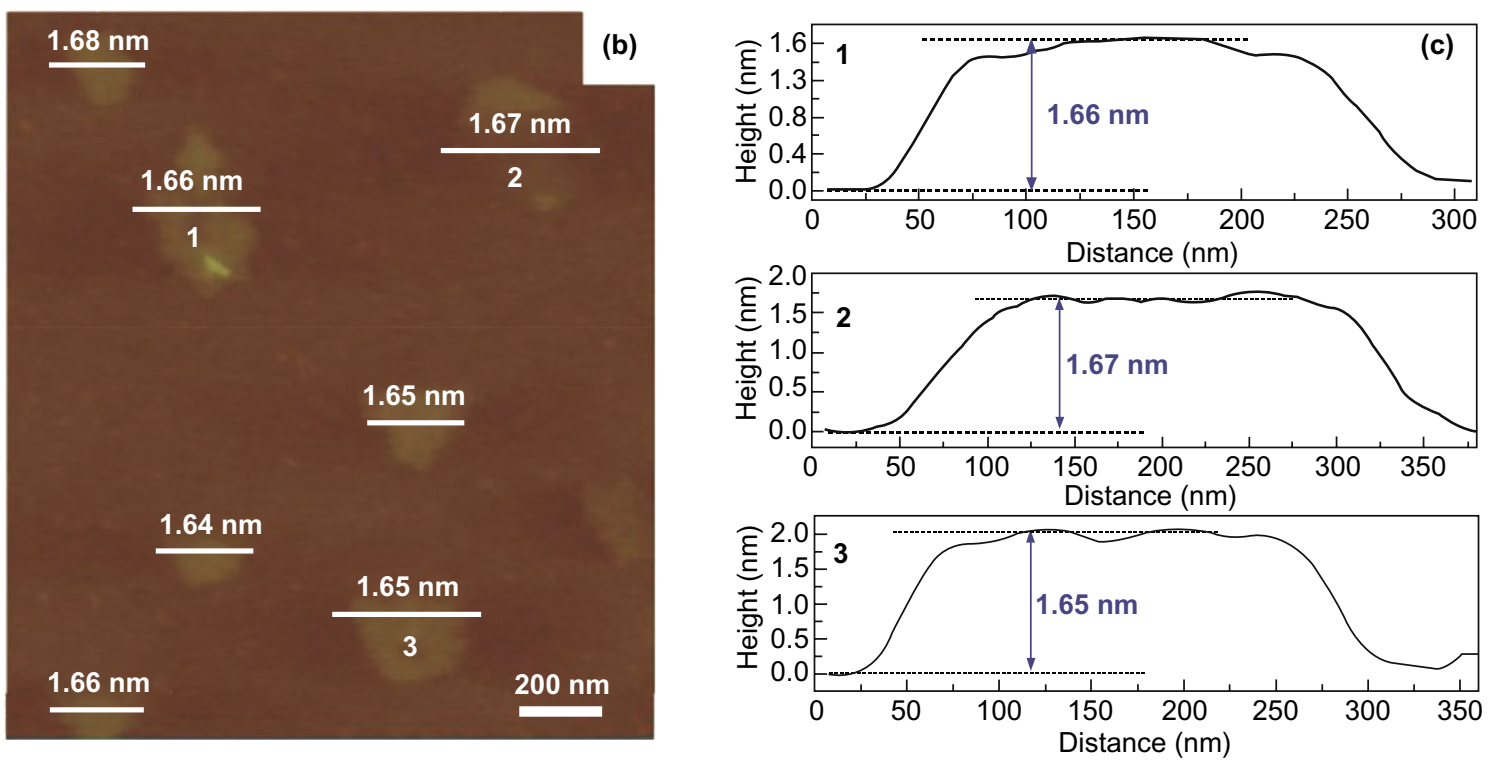

Fig. 3 The ultra-thin $\mathrm{TiO}_{2}$ flakes. a Synthesis scheme. Adapted with permission from Ref. [82]; b AFM image, $\mathbf{c}$ height profiles correspond to AFM image in b. Adapted with permission from Ref. [32] 
that $\mathrm{TiO}_{2} \mathrm{NSs}^{\prime}$ average thickness was about $1.66 \mathrm{~nm}$. A lot of other types of MOs-NSs, such as $\mathrm{Cu}_{2} \mathrm{O}$ [83] and $\mathrm{In}_{2} \mathrm{O}_{3}$ [84], were also formed via a similar technique. Taking advantage of ultra-thin size, the enhanced density of states (DOSs) by Fermi level (FL) and enhanced charge density on $\mathrm{TiO}_{2} \mathrm{NSs}$ surface were obtained. For this reason, $\mathrm{TiO}_{2} \mathrm{NSs}$ displayed quick transport of carriers and therefore achieved 450 times improved photo-catalytic activity as compared to bulk $\mathrm{TiO}_{2}$ for $\mathrm{CO}_{2}$-reduction for formate fabrication. Additionally, exfoliated single-crystalline $\mathrm{WO}_{3} \mathrm{NSs}$ were formed by $\mathrm{Bi}_{2} \mathrm{~W}_{2} \mathrm{O}_{9}$. On account of layered $\mathrm{Bi}_{2} \mathrm{~W}_{2} \mathrm{O}_{9}$ structure that is composed of $\left[\mathrm{W}_{2} \mathrm{O}_{7}\right]^{2-}$ and $\left[\mathrm{Bi}_{2} \mathrm{O}_{2}\right]^{2+}$ layers, the $\mathrm{WO}_{3}$ layers were attained through careful etching of $\left[\mathrm{Bi}_{2} \mathrm{O}_{2}\right]^{2+}$ layers via processing of acids like $\mathrm{HCL}$ and the stabilized $\mathrm{WO}_{3}$ layers can be obtained through the tetrabutylammonium hydroxide surfactant. These exfoliated $\mathrm{WO}_{3} \mathrm{NSs}$ showed an improved BG as compared with bulk- $\mathrm{WO}_{3}$, caused by quantum confinement effect. With exception of an exfoliation method, the direct preparation development of MOs-NSs was obtained by wet chemical technique. Utilizing surfactant' self-assembly approach through polyethylene oxide-polypropylene oxide-polyethylene oxide and ethylene glycol as co-surfactant, various MOs with ultra-thin thickness were formed, for instance $\mathrm{TiO}_{2}$, $\mathrm{Fe}_{3} \mathrm{O}_{4}, \mathrm{Co}_{3} \mathrm{O}_{4}, \mathrm{ZnO}, \mathrm{MnO}_{2}$, and $\mathrm{WO}_{3}$ [32].

In the last four decades, various MOs, e.g., $\mathrm{TiO}_{2}$, $\mathrm{ZnO}, \mathrm{SnO}_{2}, \mathrm{WO}_{3}$, and $\mathrm{Fe}_{2} \mathrm{O}_{3}$, were broadly examined as photo-catalysts $[42,85]$. Among them, $\mathrm{TiO}_{2}$ was the most explored one due to its good stability, biocompatibility, and favorable electronic structure as well as light absorption nature [34]. The $2 \mathrm{D}-\mathrm{TiO}_{2} \mathrm{NSs}$ obtained from the exfoliation of layered titanate have drawn attention in utilizing them as photo-catalysts [86]. The $2 \mathrm{D}-\mathrm{TiO}_{2} \mathrm{NSs}$ showed SC nature similar to their bulk cousins and include rutile and anatase form of $\mathrm{TiO}_{2}$, but with somehow superior BG because of the size quantization. For instance, $\mathrm{Ti}_{0.91} \mathrm{O}_{20.36}$-NSs exhibited a BG of $\sim 3.8 \mathrm{eV}$ that was higher than that for anatase $\mathrm{TiO}_{2}(3.2 \mathrm{eV})$ [87]. Top-down multi-step access found on intercalation and exfoliation of layered MOs was well recognized to form MO-NSs [88]. For example, for $\mathrm{TiO}_{2}-\mathrm{NSs}$, layered titanates were initially formed by high temperature, conventional solid-state reaction of $\mathrm{TiO}_{2}$, and mixture of alkali metal carbonates (Fig. 4).

After that, it was developed with an acid solution to generate protonated intermediate by ion-exchange route. The interlayers of protonated titanate were more extended by changing protons with a definite quantity of bulky organic ions, such as tetrabutylammonium cations $\left(\mathrm{TBA}^{+}\right)$. In suitable condition, layered configuration was exfoliated induced via weak shear force, for instance, mechanical shaking in aqueous solution. Different kinds of layered MOsNSs, such as $\mathrm{WO}_{3}$, titanoniobate [89] $\left(\mathrm{TiNbO}_{5}, \mathrm{Ti}_{2} \mathrm{NbO}_{7}\right.$, and $\left.\mathrm{Ti}_{5} \mathrm{NbO}_{14}\right)$, perovskite oxides [90] $\left(\mathrm{K}_{2} \mathrm{Ln}_{2} \mathrm{Ti}_{3} \mathrm{O}_{10}\right.$,

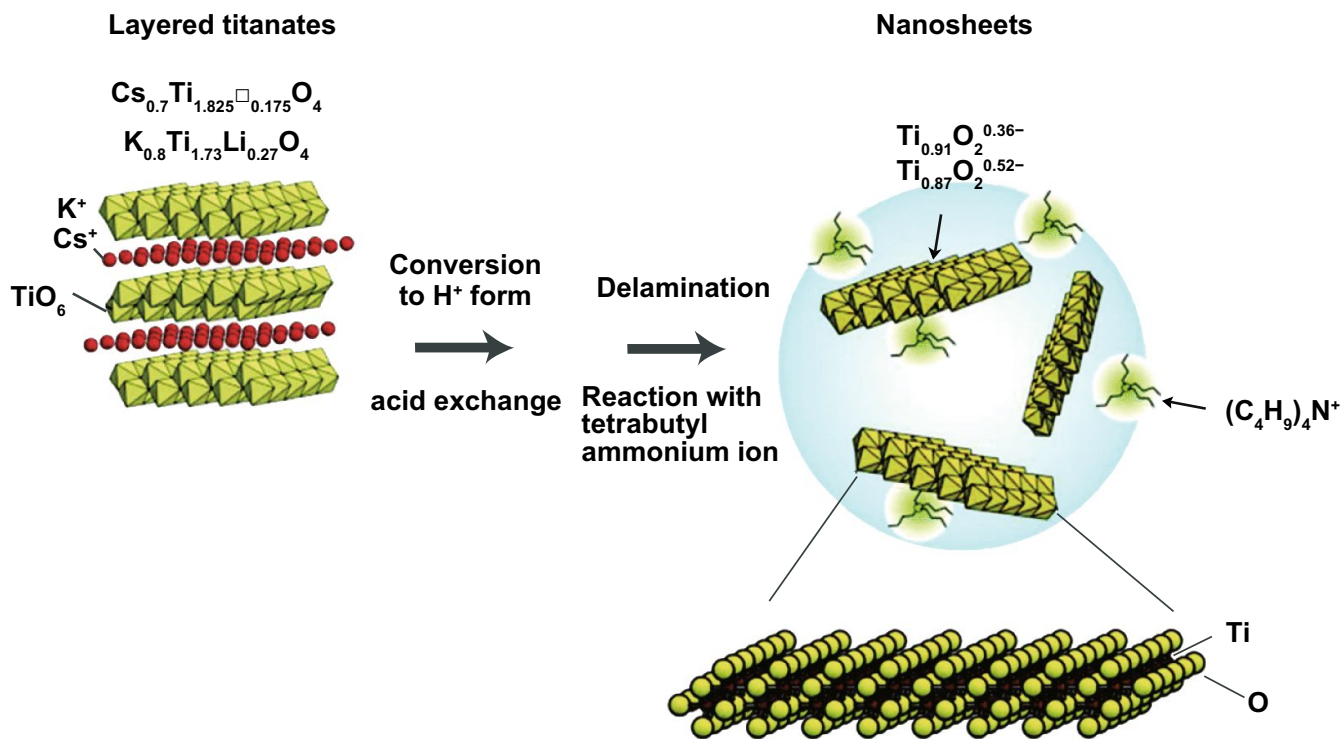

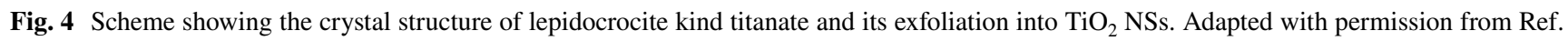
[29] 
together with $\mathrm{KLnNb}_{2} \mathrm{O}_{7}$ and $\mathrm{RbL}_{\mathrm{n}} \mathrm{Ta}_{2} \mathrm{O}_{7}$ (here $\mathrm{L}_{\mathrm{n}}$ stands for lanthanide ions)), $\mathrm{HNb}_{3} \mathrm{O}_{8}, \mathrm{HCa}_{2-x} \mathrm{SrxNb}_{3} \mathrm{O}_{10}$, and $\mathrm{HCa}_{2} \mathrm{Nb}_{3-y} \mathrm{TayO}_{10}$ [91], were formed through analogous solid-state reactions method and wet-chemical exfoliation methods. For instance, titanoniobate NSs have exhibited enhanced photo-catalytic performance in organic pollutant removal [92]. Recently, Tae et al. studied the formation of numerous diamond-shaped titanate NSs with a normal lateral size $<30 \mathrm{~nm}$, by applying a straightforward wet-chemical technique [93]. In very recent times, Zhou et al. formed freestanding, $\mathrm{SL} \mathrm{Bi}_{2} \mathrm{WO}_{6} \mathrm{MSs}$ by wet-chemical technique via using cetyltrimethylammonium bromide. Bi-atoms on SL were not saturated; hence, introduced numerous active sites on surfaces, which generated $\mathrm{h}^{+} \mathrm{s}$ directly under light irradiation. An excellent photo-catalytic performance of SL $\mathrm{Bi}_{2} \mathrm{WO}_{6}$ for photo-degradation of $\mathrm{RhB}$ was recognized by fast charge carrier separation at highly photo-active surface [94].

\subsection{Metal Composite Oxides}

Compared to MOs, metal composite oxides also showed advantages to photo-catalysis, and numerous metal composite oxides were formed with ultra-thin thickness [91]. Consistent with acid/base effect and ion intercalation supported exfoliation method, $\mathrm{HNbWO}_{6} \mathrm{NSs}$ were obtained by dispersing the layer $\mathrm{HNbWO}_{6 \cdot 1} \cdot 5 \mathrm{H}_{2} \mathrm{O}$ into tri-ethanolamine aqueous solution [95]. The results based on AFM calculations showed that $\mathrm{HNbWO}_{6} \mathrm{NSs}$ thickness was about 1.8 and $2.0 \mathrm{~nm}$, which are in agreement with SL significance. As-synthesized $\mathrm{HNbWO}_{6} \mathrm{NSs}$ suspensions displayed a proficient activity for photo-catalytic $\mathrm{H}_{2}$-evolution with a moderate rate of $158.9 \mu \mathrm{mol} \mathrm{h}^{-1}$. Furthermore, ion-exchange approaches through utilizing ultra-thin precursor were used for synthesis of metal composite oxides. For instance, $\mathrm{SnNb}_{2} \mathrm{O}_{6} \mathrm{NSs}$ were obtained through $\mathrm{K}_{4} \mathrm{Nb}_{6} \mathrm{O}_{17} \mathrm{NSs}$ and $\mathrm{SnCl}_{2}$ as precursors [96]. Through $\mathrm{K}_{4} \mathrm{Nb}_{6} \mathrm{O}_{17}$ ultra-thin thickness, it was preserved in $\mathrm{SnNb}_{2} \mathrm{O}_{6}$ with $\sim 3 \mathrm{~nm}$ thickness, as confirmed through AFM study. In comparison with bulk $\mathrm{SnNb}_{2} \mathrm{O}_{6}$, the $\mathrm{SnNb}_{2} \mathrm{O}_{6}$ NSs were having improved BG and more negative $\mathrm{CB}$ potential, denoting good reduction capability for photo-catalytic-based $\mathrm{H}_{2}$-evolution. Furthermore, charge transfer effectiveness in $\mathrm{SnNb}_{2} \mathrm{O}_{6}$ NSs was also enhanced because of ultra-thin thickness. Additional research showed that the outstanding vis-light $\mathrm{H}_{2}$-evolution activity was acquired over $\mathrm{SnNb}_{2} \mathrm{O}_{6} \mathrm{NSs}$, approximately 14 times superior to bulk $\mathrm{SnNb}_{2} \mathrm{O}_{6}$.

\subsection{Metal Hydroxides (MHOs)}

Ultra-thin MHOs were increasingly considered as significant class in 2DMs, which showed an exciting view in numerous sectors, for example catalysis, energy storage, and conversion. On account of simplicity of guideline for cations, the preferred BG was formed in MHOs by incorporating particular photo-active metal cations. So, the ultra-thin 2D-MHOs structure showed a great potential toward photo-catalytic uses. For example, ZnAl-layered double hydroxide (LDH) 2D-NSs were formed via a reverse micelle technique and used as photo-catalyst for converting $\mathrm{CO}_{2}$ to $\mathrm{CO}$ [97]. By means of sodium dodecyl sulfate as surfactant, 1-butanol as co-surfactant, translucent and stable reverse emulsion structure was created in an iso-octane $/ \mathrm{H}_{2} \mathrm{O}$ mixed solution. Following $\mathrm{Al}$ and $\mathrm{Zn}$ sources addition to mix solution, urea was used to generate alkaline condition and formed $\mathrm{ZnAl}-\mathrm{LDH}$ with ultra-thin configuration. Thickness was about $2.7 \mathrm{~nm}$ in standing NSs in TEM image and is equivalent to the thickness of 2D-LDHs' layers. Due to the ultra-thin thickness, $\mathrm{O}_{2}$ vacancies $\left(\mathrm{V}_{\mathrm{o}}\right)$ were formed in ultra-thin $\mathrm{ZnAl}-\mathrm{LDH}$ NSs, resulting in the creation of $\mathrm{Zn}^{+}-\mathrm{V}_{\mathrm{o}}$ complexes. The DFTbased study showed a novel defect-level hybridization with both occupied $\mathrm{Zn} 4 s$ orbitals and $\mathrm{O}_{2 p}$ orbitals emerging in BG of ultra-thin ZnAl-LDH NSs compared with bulk ZnAl$\mathrm{LDH}$. The $\mathrm{Zn}^{+}-\mathrm{V}_{\mathrm{o}}$ complexes can provide $\mathrm{e}^{-}$trap sites for $\mathrm{CO}_{2}$ photo-reduction. Consequently, an appreciably amplified photo-catalytic activity for $\mathrm{CO}_{2}$-reduction was obtained for ultra-thin ZnAl-LDH NSs compared with bulk ZnAlLDH. Except ZnAl-LDH, several new MHOs with ultra-thin thickness, for example $\mathrm{CoOOH}$ [98], NiTi-LDH, and ZnTi LDH [99], also showed wonderful performance for diverse photo-catalytic uses.

\subsection{Metal Chalcogenides}

The TMDs have gained much interest because their mechanical, optical, and electrical characteristics were explored for a wide range of applications, for instance biosensors, catalysis, lithium battery cathodes, transistors, memory devices, photovoltaics, photodetectors, photo-catalytic solid lubricants, and PEC conversions. The TMDs (e.g., $\mathrm{MoS}_{2}, \mathrm{WS}_{2}$, and 
(a)

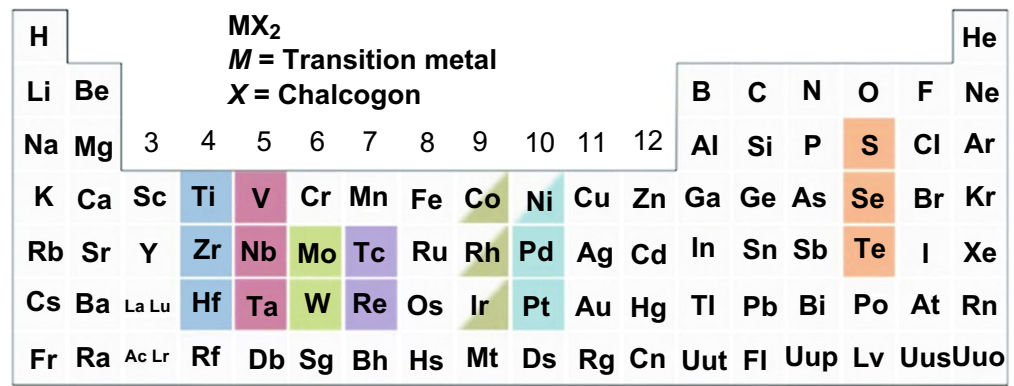

(b)

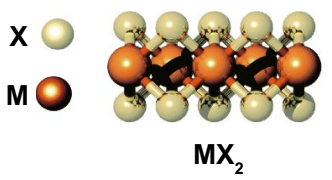

(c)

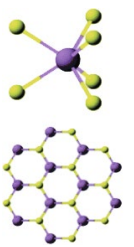

$2 \mathrm{H}$

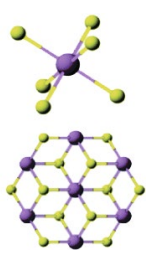

$1 \mathrm{~T}$

Fig. 5 a Transition metals and three chalcogen elements (yellow color) which are composed of TMD layer structures. b TMD structures. c $\mathrm{MoS}_{2}$ two-phase (1T, 2H) structures. Adapted with permission from Ref. [100]

$\mathrm{TiSe}_{2}$ ) are a large group of layered materials with common representation as " $\mathrm{MX}_{2}$," where $\mathrm{M}$ is a transition metal element of group 4-10 ((Ti, Hf, Zr), (Ta, V, Nb), and (W, Mo)) and $X$ is the chalcogen atom ( $\mathrm{S}, \mathrm{Se}, \mathrm{Te})$. The TMD NSs have different functions in PC and PEC applications. They behave as a photo-sensitizer via increasing light harvest ability in vis region of sun irradiation, a charge separator throughout appropriate energy band arrangement, and a charge carrier. Correct function of 2D nanosheets (NSs) depends on utilization of reaction scheme. In consequence of special electronic configuration, in general metal chalcogenides showed a comparatively broad light absorption area (Fig. 5), which were measured to be a group of talented materials having photocatalytic uses. Normally, the stoichiometry of TMDs can be expressed through formula $\mathrm{MX}_{2}$, in which $\mathrm{M}$ and $\mathrm{X}$ signify a chalcogen and a transition metal, respectively. The single layer of a TMD involves three atoms, where $\mathrm{M}$ is situated in two $\mathrm{X}$ (Fig. 5a, b). The configurations of $1 \mathrm{~T}$ and $2 \mathrm{H}$ phases of $\mathrm{MoS}_{2}$ are shown in Fig. 5c.

Recently, many 2D metal chalcogenides have been formed and showed an excellent photo-catalytic performance, e.g., CdS, $\mathrm{MoS}_{2}, \mathrm{SnS}_{2}, \mathrm{SnS}_{2} \mathrm{In}_{2} \mathrm{~S}_{3}, \mathrm{ZnIn}_{2} \mathrm{~S}_{4}, \mathrm{ZnSe}$, and $\mathrm{SnSe}$ [101]. The synthetic techniques of such 2D metal chalcogenides generally concentrate on exfoliation, because of essential layer structures. Taking hexagonal $\mathrm{SnS}_{2}\left(\mathrm{~h}-\mathrm{SnS}_{2}\right)$, such as h-SnS 2 SLs, can be attained through refluxing bulk-SnS $\mathrm{S}_{2}$ in formamide to rupture interlayer
vdWs' interactions [102]. Almost transparent aspect of $\mathrm{SnS}_{2}$ NSs in TEM image exposes ultra-thin thickness, and it was described to be $0.61 \mathrm{~nm}$ via AFM, well matched with SL-SnS ${ }_{2}$ slab along [001] direction. As a result of SL configuration, electronic structure of $\mathrm{SLs}_{-} \mathrm{SnS}_{2}$ experienced discrete changes, with increased BG, higher DOSs at VB edge, and faster interfacial charge transfer. Therefore, SLs$\mathrm{SnS}_{2}$ delivers a surprisingly improved photo-catalytic $\mathrm{H}_{2} \mathrm{O}$ splitting activity (70 times development) compared to bulk$\mathrm{SnS}_{2}$, under vis-light. The 2D metal chalcogenide NSs, for example $\mathrm{MoS}_{2}$ [103], $\mathrm{SnS}_{2}$ [104], $\mathrm{TiS}_{2}$ [105], $\mathrm{WS}_{2}$ [106], $\mathrm{MoSe}_{2}$ [107], $\mathrm{WSe}_{2}$ [103], etc., are rising as a new significant class of 2DMs in the photo-catalysis applications because of their good electronic properties [108]. Taking $\mathrm{MoS}_{2}$ as an example, bulk-MoS${ }_{2}$ materials have indirect $\mathrm{BG}$ of $1.2 \mathrm{eV}$ that is not suitable for photo-catalytic reactions caused by the lack of oxidation or reduction potential $\left(E_{0}\right)$ for activating photo-catalytic method. However, $\mathrm{MoS}_{2}$-NSs had been established with having a direct BG of $\sim 1.96 \mathrm{eV}$ because of quantum confinement effect that provides $\mathrm{MoS}_{2} /$-NSs along appropriate band positions and capability for vis-light absorption. In contrast to most of the layer MOs, vdWs bonding of metal chalcogenide interlayers creates exfoliation of these layers easily. Until now, a lot of top-down approaches are described for the formation of SL or FL metal chalcogenide NSs, for example lithium intercalation-exfoliation, mechanical exfoliation, 
and liquid phase ultrasonic exfoliation [109]. Furthermore, bottom-up chemical production and chemical vapor deposition (CVD) techniques suggested potential influential alternatives such as exfoliation techniques for fabricating metal chalcogenide NSs. For example, Cheon et al. introduced disk-shaped $\mathrm{ZrS}_{2} \mathrm{NSs}$ with $<2 \mathrm{~nm}$ thicknesses and lateral size ranging from 20 to $60 \mathrm{~nm}$ via reacting $\mathrm{ZrCl}_{4}$ and $\mathrm{CS}_{2}$ in oleylamine [110]. This method was, soon after that, extended for other transition metal selenide and sulfide NSs. The $\mathrm{MoS}_{2}$-NSs were formed via solvothermal techniques using $\left(\mathrm{NH}_{4}\right)_{6} \mathrm{Mo}_{7} \mathrm{O}_{24} \cdot 4 \mathrm{H}_{2} \mathrm{O}$ and thiourea as precursors [111].

\subsection{Bismuth-Based Materials}

Recently, bismuth (Bi)-based materials have been broadly investigated and studied for their photo-catalysis applications because $\mathrm{Bi} 6 \mathrm{~s}$ in $\mathrm{Bi}(\mathrm{III})$ can hybridize with $\mathrm{O}_{2 p}$ orbitals to generate novel favorable hybridized VB and BG of Bi-based materials which are narrowed for absorption of (a)
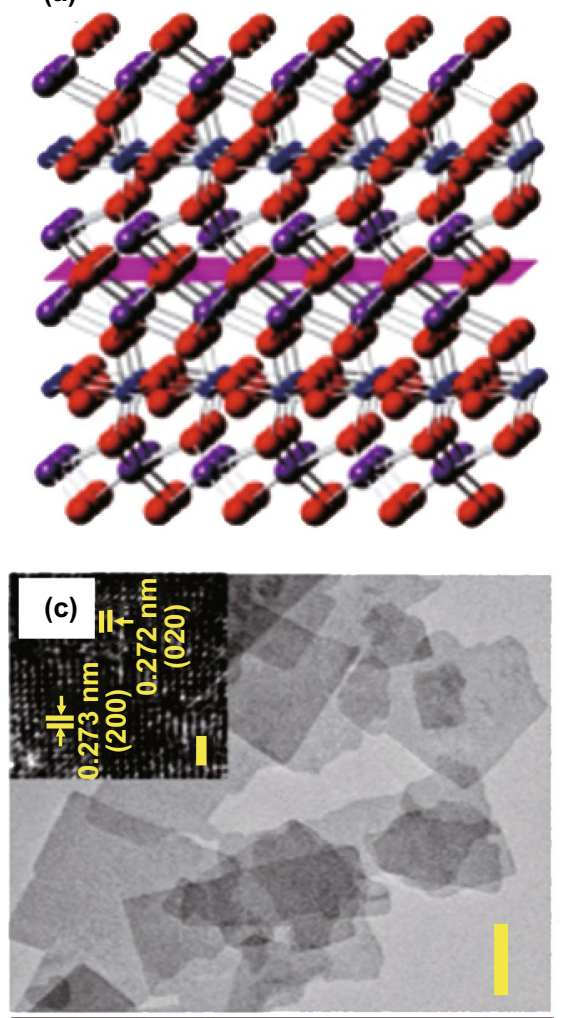

\section{(d)}

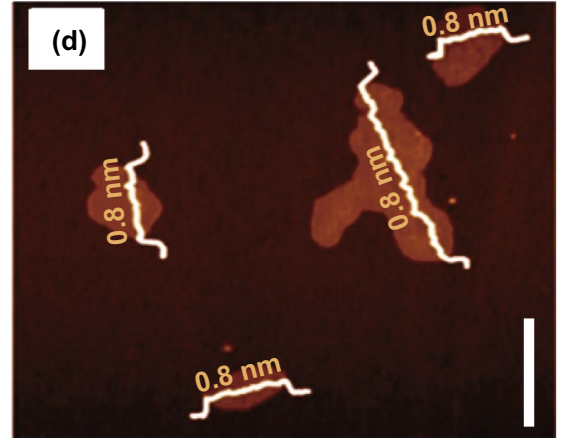

(b)
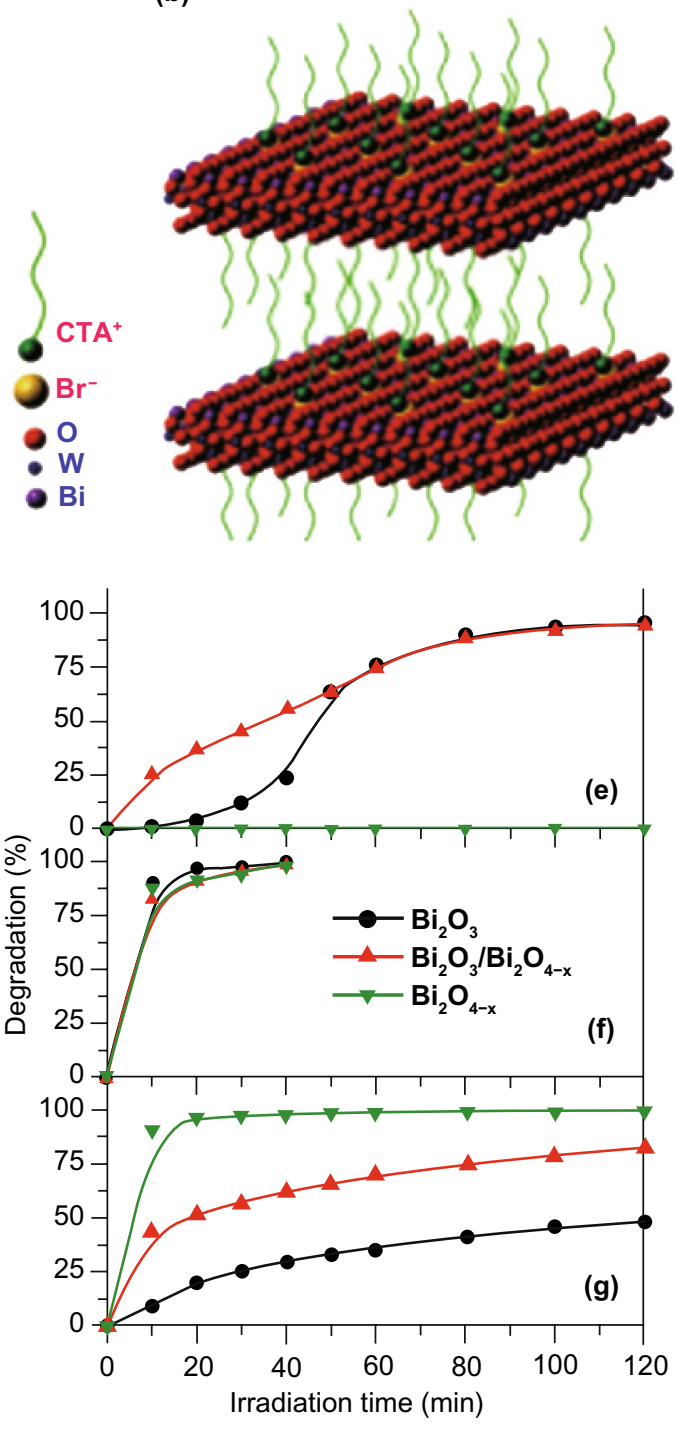

Fig. 6 a $\mathrm{Bi}_{2} \mathrm{WO}_{6}$ crystal structure. b Fabrication method of the $\mathrm{SL} \mathrm{Bi}_{2} \mathrm{WO}_{6}$ with $\mathrm{CTAB}$ support. $\mathbf{c} \mathrm{TEM} / \mathrm{HR}-\mathrm{TEM}$ of $\mathrm{Bi}_{2} \mathrm{WO}_{6}$ formed by $\mathrm{CTAB}$ support. d AFM analysis of $\mathrm{SL} \mathrm{Bi}_{2} \mathrm{WO}_{6}$ based on CTAB. Adapted with permission from Ref. [32]. Comparison of photo-catalytic degradation of e MB, $\mathbf{f} \mathrm{MO}$, and $\mathbf{g}$ phenol over $\mathrm{Bi}_{2} \mathrm{O}_{3}, \mathrm{Bi}_{2} \mathrm{O}_{3} / \mathrm{Bi}_{2} \mathrm{O}_{4-x}$, and $\mathrm{Bi}_{2} \mathrm{O}_{4-x}$. Adapted with permission from Ref. [118] 
vis-light. Due to continuous improvement in photo-catalytic performance, numerous Bi-based materials with controlled ultra-thin thickness are formed, for example $\mathrm{Bi}_{2} \mathrm{WO}_{6}$ [112, 113], $\mathrm{Bi}_{2} \mathrm{MoO}_{6}$ [85], $\mathrm{BiVO}_{4}$ [114], $\mathrm{Bi}_{2} \mathrm{SiO}_{5}$ [115] ( $\mathrm{BiO})_{2} \mathrm{CO}_{3}$ [94], $\mathrm{Bi}_{3} \mathrm{NbO}_{7}$ [116], $\mathrm{BiOX}(\mathrm{X}=\mathrm{Cl}, \mathrm{Br}$, I) [117], and $\mathrm{Bi}_{2} \mathrm{O}_{3} / \mathrm{Bi}_{2} \mathrm{O}_{4-x}$ nano-composite [118]. The $\mathrm{SL} \mathrm{Bi}_{2} \mathrm{WO}_{6} \mathrm{NSs}$ were formed via surfactant cetyltrimethylammonium bromide (CTAB)-supported hydrothermal technique [94], where $\mathrm{Br}$-ions from CTAB were adsorbed at $\mathrm{SL} \mathrm{Bi}_{2} \mathrm{WO}_{6}$ surface and produced Coulomb repulsion forces, which delayed stacking of $\mathrm{SLs}_{\mathrm{Bi}_{2}} \mathrm{WO}_{6}$. Furthermore, hydrophobic long-chain cationic $\mathrm{CTA}^{+}$at $\mathrm{Bi}_{2} \mathrm{WO}_{6}$ surface supplied an extra surface repulsion to further stop crystal growth along the c-axis. Therefore, $\mathrm{SL} \mathrm{Bi}_{2} \mathrm{WO}_{6}$ slab $(0.8 \mathrm{~nm}$ thickness $)$ with $[\mathrm{BiO}]^{+}-\left[\mathrm{WO}_{4}\right]^{2-}-[\mathrm{BiO}]^{+}$ sandwich substructure was achieved, as supported from AFM analysis (Fig. 6a-d). Plentiful coordinative unsaturated $\mathrm{Bi}$-atoms were exposed at $\mathrm{SL}_{2} \mathrm{Bi}_{2} \mathrm{WO}_{6} \mathrm{SNs}$ and act as active sites. After irradiation with light, $\mathrm{h}^{+} \mathrm{s}$ is produced in $[\mathrm{BiO}]^{+}$as $\mathrm{e}^{-} \mathrm{s}$ in $\left[\mathrm{WO}_{4}\right]^{2-}$. Resembling hetero-junction interface, sandwich $[\mathrm{BiO}]^{+}-\left[\mathrm{WO}_{4}\right]^{2-}-[\mathrm{BiO}]^{+}$substructure permits efficient interface for space charge separation.

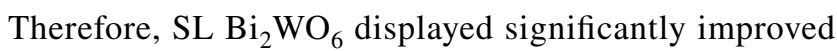
photo-catalytic activity toward pollution deduction in vis-light.

In addition, as-synthesized $\mathrm{SL} \mathrm{Bi}_{2} \mathrm{WO}_{6}$ illustrated $\mathrm{H}_{2}$-evolution activity under vis-light, even if $\mathrm{Bi}_{2} \mathrm{WO}_{6}$ nanocrystal in fact holds no $\mathrm{H}_{2}$-evolution activity. Altering the surfactant type to polyvinylpyrrolidone (PVP), ultra-thin $\mathrm{BiOCl}$ NSs were attained by means of a solvothermal treatment [119]. The BiOCl nanoplate's thickness $(\approx 30 \mathrm{~nm})$ was reduced to $2.7 \mathrm{~nm}$, while PVP was applied as a capping agent, as verified through the AFM analysis. The polyvinyl skeleton structure of PVP prevented more development of the $\mathrm{BiOCl}$ nano-crystal by generating passivation layer about $\mathrm{BiOCl}$ cores via strong interaction with $\mathrm{Bi}^{3+}$, $\mathrm{N}$, and $\mathrm{O}$ atoms of pyrrolidone ring. This suppressed the agglomeration of $\mathrm{BiOCl}$ nano-crystal along c-axis during repulsion forces in between polyvinyl groups. Therefore, ultra-thin BiOCl NSs were prepared via the PVP-assisted solvothermal treatment and this method was further used for synthesis of ultra-thin $\mathrm{BiOBr}$ and $\mathrm{BiOI} 2 \mathrm{D}$ form [120]. The attained ultra-thin thicknesses give BiOCl NSs with upshifted CB and VB potentials and reduced the BG relative to $\mathrm{BiOCl}$ nano-plates. As such, effective division of photo-induced $\left(\mathrm{e}^{-}-\mathrm{h}^{+}\right)$-pairs was obtained and caused an increase in photo-catalytic activity for pollutant removal [32]. Paolo Fornasiero and co-workers also explored $\mathrm{Bi}_{2} \mathrm{O}_{3} /$ $\mathrm{Bi}_{2} \mathrm{O}_{4-x}$ composite that functions as a potential photo-catalyst (Fig. 6e-g). The aim of the study was to begin active species on photo-catalyst surface via utilizing a non-traditional advancement. Therefore, they utilized (UV-Vis)light to stimulate alterations in $\mathrm{Bi}_{2} \mathrm{O}_{3}$ surface that produces $\mathrm{Bi}_{2} \mathrm{O}_{3} / \mathrm{Bi}_{2} \mathrm{O}_{4-x}$ nano-composite arrangement. So, for methylene blue (MB) such surface modifications bring significant enhancement in photo-catalytic performance. The wide $\mathrm{BG}$ with respect to $\mathrm{Bi}_{2} \mathrm{O}_{3}$ along excitation considerations proposes that analogous photo-induced crystal modifications, although exist, should be insignificant for $\mathrm{TiO}_{2}$-based materials. Until now, only careful designed thermal treatments were able to make exciting anatase/ rutile nano-composites with outstanding photo-catalytic performance [118].

\subsection{Metal-Free NSs}

Excluding metal containing SC 2DMs, metal-free 2DMs were also formed as photo-catalysts. Recently, new classes of metal-free 2DMs have been introduced from lightweight and abundant elements, such as carbon, phosphorus, and binary carbon nitride, boron carbide, and hexagonal boron nitride (h-BN) that reveal new prospects for photochemistry. The $2 \mathrm{D}-\mathrm{G}$ with hexagonal $s p^{2}$-hybridized structure is inspiring great research concern in a range of energy-related uses because of its elevated carrier mobility. The high flexibility and larger SSA alone from accessibility of solution processable graphene oxide (GO) allows 2D-G NSs to simply merge with other SCs to form electronic bridges [121]. Recently, 2D-G have been intensively studied in photocatalytic fields and demonstrated as competent $\mathrm{e}^{-}$-acceptor to improve the charge transfer and reduce $\left(\mathrm{e}^{-}-\mathrm{h}^{+}\right)$-pair's recombination to improve photo-catalytic activity of composite photo-catalysts [3, 4, 18, 19]. More interestingly, although SL 2D-G is recognized as a semimetal with a zero $\mathrm{BG}$, which is not suitable in light absorption, numerous scientists have confirmed that functionalized $2 \mathrm{D}-\mathrm{G}$ base analogy like 2D-GO could be promising materials for nonmetal photo-catalysts as band structure of GO is associated with its degree of oxidation that can be engineered via choosing appropriate preparation methods. For example, Yeh et al. observed a 2D-GO that could work as active photo-catalyst 
(a)
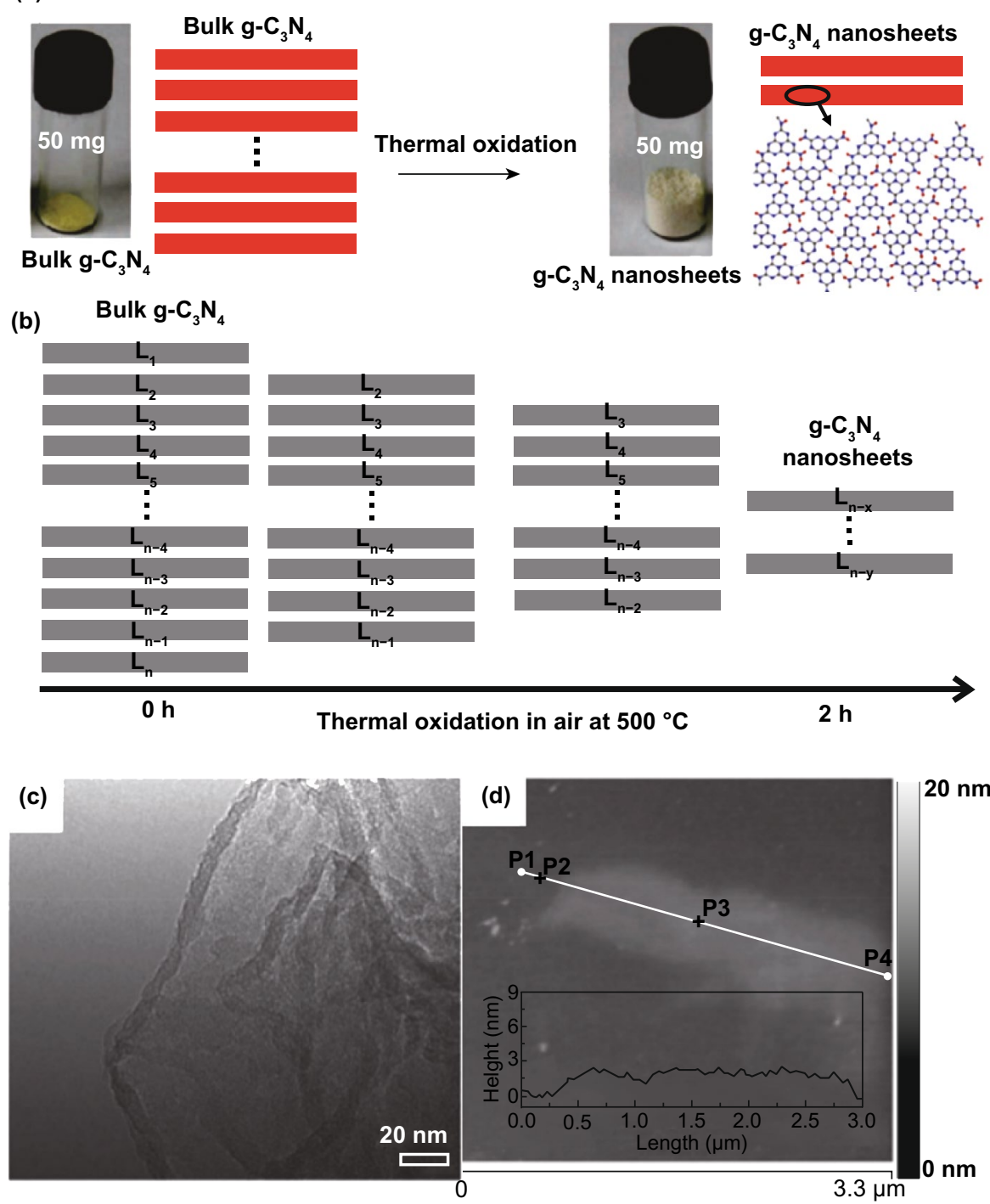

Fig. 7 a Scheme shows bulk g- $\mathrm{C}_{3} \mathrm{~N}_{4}$ and $\mathrm{g}-\mathrm{C}_{3} \mathrm{~N}_{4}$ NS structures. Adapted with permission from Ref. [29]. b Scheme to fabricate g- $\mathrm{C}_{3} \mathrm{~N}_{4}-\mathrm{NSs}$ through thermal oxidation etching of bulk $\mathrm{g}_{-} \mathrm{C}_{3} \mathrm{~N}_{4}$ at $500{ }^{\circ} \mathrm{C}$ in air. c TEM image, d AFM analysis of g- $\mathrm{C}_{3} \mathrm{~N}_{4}-\mathrm{NSs}$. Adapted with permission from Ref. [32]

in $\mathrm{H}_{2} \mathrm{O}$ splitting [122] and can gradually produce $\mathrm{H}_{2}$ from $20 \mathrm{vol} \%$ methanol solution in $\mathrm{H}_{2} \mathrm{O}$ and pristine $\mathrm{H}_{2} \mathrm{O}$ after irradiation with UV/Vis-light. After that, Hsu et al. studied 2D-GO and showed an elevated photo-catalytic efficiency for transformation of $\mathrm{CO}_{2}$ to methanol $\left(\mathrm{CH}_{3} \mathrm{OH}\right)$ by solar light irradiation [123]. As an equivalent of $2 \mathrm{D}-\mathrm{G}, \mathrm{g}_{-} \mathrm{C}_{3} \mathrm{~N}_{4}$ NSs were rapidly rising because of their excellent chemical and electronic properties [124]. Bulk g- $\mathrm{C}_{3} \mathrm{~N}_{4}$ has a layered $2 \mathrm{D}$ configuration and proper $\mathrm{BG}(\sim 2.7 \mathrm{eV})$ for light absorption in visible range. The $\mathrm{g}^{-} \mathrm{C}_{3} \mathrm{~N}_{4} \mathrm{NSs}$ were obtained through delaminating bulk layered $\mathrm{g}-\mathrm{C}_{3} \mathrm{~N}_{4}$ that is usually formed via pyrolysis of $\mathrm{N}_{2}$-rich precursors through bulk reaction or polycondensation.

A new metal-free photo-catalyst, with outstanding photocatalytic proficiency of $\mathrm{g}^{-} \mathrm{C}_{3} \mathrm{~N}_{4} \mathrm{NSs}$ under vis-light irradiation, was verified in many photo-catalytic uses. For example, Niu et al. [125] studied a simple top-down approach to form g- $\mathrm{C}_{3} \mathrm{~N}_{4} \mathrm{NSs}$ via oxidation etching of bulk g- $\mathrm{C}_{3} \mathrm{~N}_{4}$ in air under high temperature (Fig. 7). The acquired $\mathrm{g}_{-} \mathrm{C}_{3} \mathrm{~N}_{4} \mathrm{NSs}$ thickness was about $2 \mathrm{~nm}$ with SSA $306 \mathrm{~m}^{2} \mathrm{~g}^{-1}$, which was high in comparison with bulk phase. Quantum confinement effect causes enhanced $\mathrm{e}^{-}$-transfer ability toward in-plane 
direction, and the lifetime of photo-generated charge carriers was improved. Therefore, photo-catalytic performance of g- $\mathrm{C}_{3} \mathrm{~N}_{4} \mathrm{NSs}$ for $\mathrm{H}_{2}$-production process was really enhanced. In recent times, other liquid phase exfoliation techniques are formed to synthesize $\mathrm{g}_{-} \mathrm{C}_{3} \mathrm{~N}_{4}$ NSs from bulk counterpart. For instance, Yang et al. prepared freestanding g- $\mathrm{C}_{3} \mathrm{~N}_{4} \mathrm{NSs}$ through liquid phase exfoliation of $\mathrm{g}-\mathrm{C}_{3} \mathrm{~N}_{4}$ powder in isopropanol; this exhibited good photo-catalytic effectiveness for $\mathrm{H}_{2}$-evolution by applying vis-light irradiation. Photocatalytic efficiency of exfoliated NSs was higher $>17$ factor contrast to non-exfoliated counterpart and with a factor of $>8$ than already described $\mathrm{g}-\mathrm{C}_{3} \mathrm{~N}_{4} \mathrm{NSs}$ [126]. Apart from distinctive energy band configuration, $\mathrm{g}_{-} \mathrm{C}_{3} \mathrm{~N}_{4}$ is more active toward many photo-catalytic uses, for example $\mathrm{H}_{2}$-evolution, $\mathrm{CO}_{2}$-reduction, pollutant deduction, disinfection, etc. As a result of in-plane graphite-like layer configuration with strong $\mathrm{C}-\mathrm{N}$ covalent bonding and interlayer weak vdWs' forces, bulk g- $\mathrm{C}_{3} \mathrm{~N}_{4}$ was accountable to be exfoliated and obtained in FL or even SL form. Generally, there are two methods for $\mathrm{g}-\mathrm{C}_{3} \mathrm{~N}_{4}$ exfoliation, i.e., thermal oxidation and ultra-sonication-based liquid exfoliation techniques. In view of that, $\mathrm{H}_{2}$-bond coherent strands of polymeric melon units in layers were not sufficiently stable beside oxidation. Liu et al. [125] formed a thermal oxidation exfoliation method to form an ultra-thin $\mathrm{g}-\mathrm{C}_{3} \mathrm{~N}_{4} \mathrm{NSs}$. Thicknesses of bulk g- $\mathrm{C}_{3} \mathrm{~N}_{4}$ were steadily reduced with increasing times through layerby-layer etching method (Fig. 7b-d). After 120 min, thermal oxidated $\mathrm{g}_{-} \mathrm{C}_{3} \mathrm{~N}_{4} \mathrm{NSs}$ with almost $2 \mathrm{~nm}$ thicknesses were obtained. Since quantum confinement effect and increased $\mathrm{BG}$ promoted $\mathrm{e}^{-} \mathrm{s}$ migration rates along in-plane direction, $\mathrm{H}_{2}$-evolution activity improved 5.4 times, as compared to bulk counterpart. Encouraged by this synthesis method, numerous advance researches achieved organizing ultrathin $\mathrm{g}_{-} \mathrm{C}_{3} \mathrm{~N}_{4}$-NSs via modified techniques $[127,128]$. The ultra-sonication assisted liquid exfoliation was observed as another effective technique to attain ultra-thin $\mathrm{g}_{-} \mathrm{C}_{3} \mathrm{~N}_{4} \mathrm{NSs}$ because of fundamentally layered structure.

In exfoliation process, the efficiency is affected due to surface energies, and when solvent and bulk materials match each other, exfoliation was extremely good. The calculated g- $\mathrm{C}_{3} \mathrm{~N}_{4}$ surface energy was almost $115 \mathrm{~mJ} \mathrm{~m}^{-2}$, in a good agreement with $\mathrm{H}_{2} \mathrm{O}\left(\sim 10^{2} \mathrm{~mJ} \mathrm{~m}^{-2}\right)$. Thus, with the use of $\mathrm{H}_{2} \mathrm{O}$ in $\mathrm{g}_{-} \mathrm{C}_{3} \mathrm{~N}_{4}$ liquid exfoliation, exfoliated NSs with almost $2.5 \mathrm{~nm}$ thickness were acquired [129]. Based on the analogous law, Ajayan and co-workers [130] calculated g- $\mathrm{C}_{3} \mathrm{~N}_{4}$ exfoliated by isopropanol. The as-formed $\mathrm{g}_{-} \mathrm{C}_{3} \mathrm{~N}_{4}$-NSs were having homogeneous thickness of $\sim 2 \mathrm{~nm}$. This ultra-thin thickness of $\mathrm{g}_{-} \mathrm{C}_{3} \mathrm{~N}_{4}$-NSs displayed an improved $\mathrm{BG}$ as well as charge migration rate in contrast to bulk $\mathrm{g}-\mathrm{C}_{3} \mathrm{~N}_{4}$. This caused a 9.3 times higher photo-catalytic-based $\mathrm{H}_{2}$-evolution. Similarly, other solvents were also searched to form ultra-thin $\mathrm{g}-\mathrm{C}_{3} \mathrm{~N}_{4}$ and obtained improved photocatalytic performance [131]. Despite g- $\mathrm{C}_{3} \mathrm{~N}_{4}$, other metalfree materials were also formed, which act as photo-catalysts for different uses [132]. For example, ultra-thin silicon NSs controlled synthesis via molten salt-induced exfoliation and chemical reduction of natural clay [133]. Ultra-thin silicon NSs $\left(\approx 5 \mathrm{~nm}\right.$ thickness) showed an excellent $\mathrm{H}_{2}$-evolution performance from a $\mathrm{H}_{2} \mathrm{O}$ methanol mixture. Advanced investigations showed that proficient $\mathrm{H}_{2} \mathrm{O}$ splitting was obtained over ultra-thin silicon NSs lacking addition of co-catalyst or sacrificial agent [134]. Unfortunately, ultra-thin silicon NSs have experienced serious activity decline by extended time. How to approve suitable approach to increase stability might be heart of $\mathrm{H}_{2} \mathrm{O}$ splitting over ultra-thin silicon NSs [32].

\subsection{Other Metal Containing 2D-NSs}

In addition to aforementioned different types of materials, other recently introduced 2DMs, for example layered metal oxy-nitride and oxy-halides, and metal carbides, also have a great potential for photo-catalysis uses after chemical doping or combining with other SCs. For instance, bismuth oxy-halides $\left(\mathrm{BiO}_{\mathrm{X}}, \mathrm{X}=\mathrm{Cl}, \mathrm{Br}\right.$, and $\left.\mathrm{I}\right)$ were gotten increasing interest because of their outstanding photo-catalytic nature, that are analogous to or even greater than those of the anatase $\mathrm{TiO}_{2}$ [119]. Moreover, neutral layers of the $\mathrm{Ti}_{3} \mathrm{C}_{2}(\mathrm{OH})_{2}$ formed through HF-assisted exfoliation of metal carbides, for example $\mathrm{Ti}_{3} \mathrm{AlC}_{2}$, were verified as competent photo-catalyst for adsorption and photo-catalytic decomposition of organic molecules in an aqueous atmosphere [135].

\subsection{D/2D Hetero-structures}

Properly developing the 2D/2D hetero-structures confirmed the most talented form for further boosting the photo-catalytic activity, because of that hetero-junction interfacial effect [136]. The hetero-junction interfacial effect can encourage separation and therefore extend lifetime of the photo-generated $\left(\mathrm{e}^{-}-\mathrm{h}^{+}\right)$-pairs in catalyst that directly or indirectly contributes to redox reaction of photo-catalytic 
$\mathrm{H}_{2}$-production or organics degradation. Several attempts were applied to engineer 2D-component or reinforce the interfacial acting force to form the capable 2D/2D photocatalysts. Although hetero-junction found on a range of dimensions (e.g., 2D/2D, 3D/3D, and 2D/3D) with exposed interface put right contact, they are all possible efficient catalysts. The 2D/2D hetero-junctions have different advantages for catalysis, as follows:

1. High catalytic active sites because of great SSA/interface area and ultra-thin thickness.

2. Charges are easily transferred because of small basic resistance and short transport path in $1 \mathrm{D}$ of the ultra-thin $2 \mathrm{D}$ components.

3. Transparency consequence from ultra-thin thickness is helpful in light absorption.

Therefore, plan as well as the use of 2D/2D layered hetero-structures has rapidly become the most up-to-date research topics. Recently, the family of ultra-thin, 2D layered materials formed significantly. Further, other than presently used ultra-thin 2D-NSs, for example 2D-G, TMDCs, and noble metals, there are many other 2D-NSs with changeable electronic and physical properties formed from the last few years, e.g., MOs, h-BN, black phosphorus, metal-organic frameworks (MOFs), organic crystals, and covalent organic frameworks (COFs) [136].

\section{Electronic Structure Engineering}

Engineering an electronic configuration, the properties of ultra-thin 2DMs are experiencing a different variation, offering probabilities to enhance or even provoke novel photocatalytic activity. There are a range of ways to engineer the electronic structure of ultra-thin 2DMs, e.g., thickness tuning, component tuning, defect engineering, doping, and so (a)

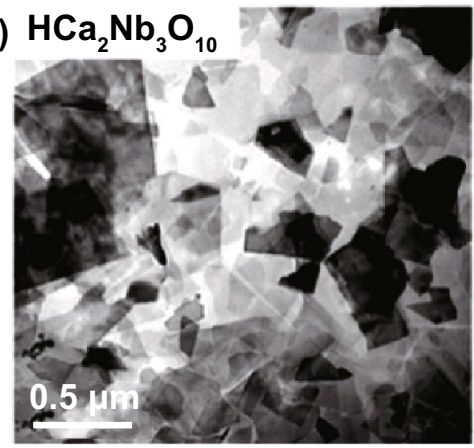

(d)

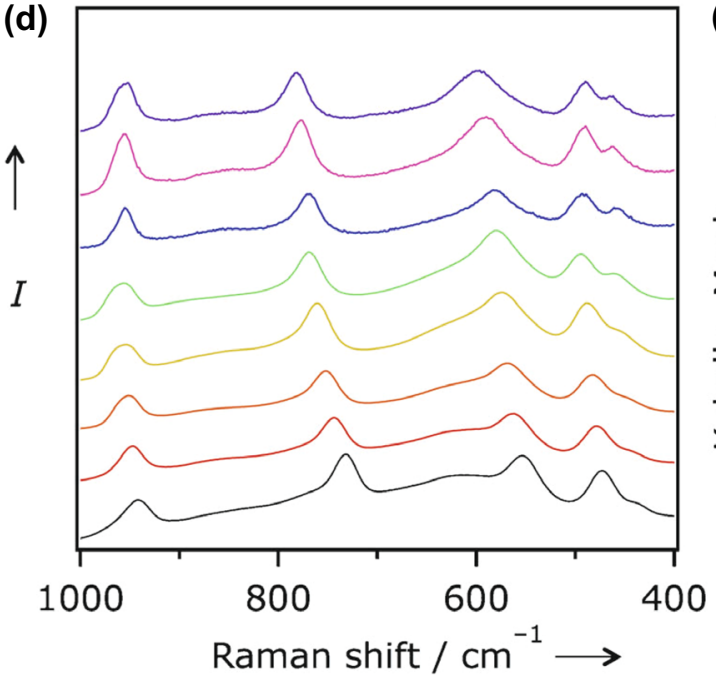

(b) $\mathrm{HCaSrNb}_{3} \mathrm{O}_{10}$
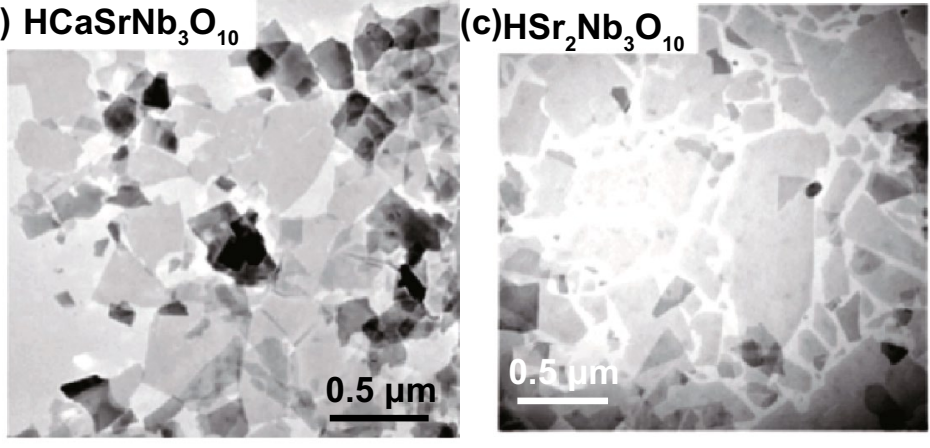

(e)

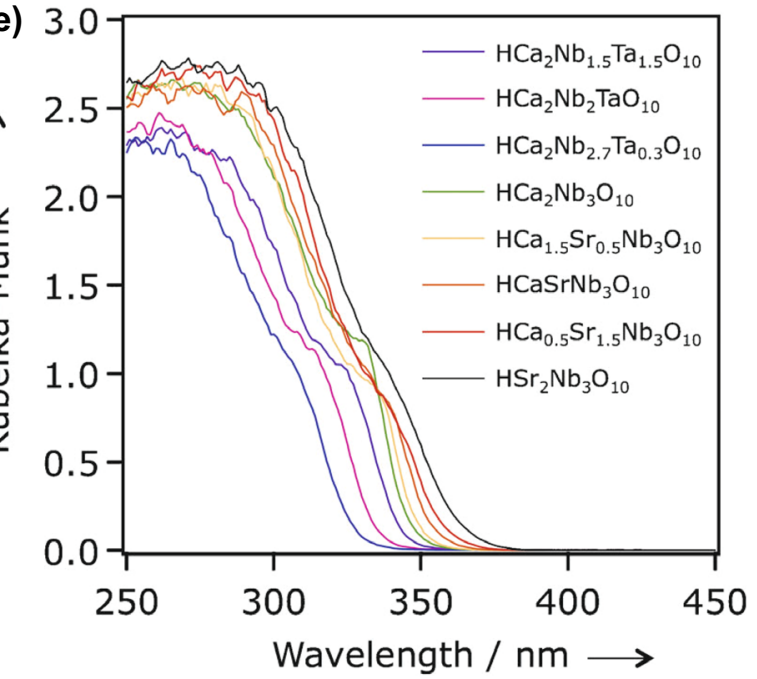

Fig. 8 a-c TEM results of TBA ${ }^{+}$-exfoliated $\mathrm{Ca}_{2-x} \mathrm{Sr}_{x} \mathrm{Nb}_{3} \mathrm{O}_{10}$-NSs. d, e Raman spectra and UV-Vis diffuse reflectance spectrum of restacked $\mathrm{HCa}_{2-x} \mathrm{Sr}_{x} \mathrm{Nb}_{3} \mathrm{O}_{10}$ and $\mathrm{HCa}_{2} \mathrm{Nb}_{3-y} \mathrm{Ta}_{y} \mathrm{O}_{10} \mathrm{NSs}$. Adapted with permission from Ref. [137] 
on. Now, we will explain most of them in detail in the next section.

\subsection{Component Tuning}

For photo-catalytic employment, component of SC establishes band configuration. As reactivity of the photo-generated $\mathrm{e}^{-} \mathrm{s}$ and $\mathrm{h}^{+} \mathrm{s}$ toward resultant surface redox reactions was usually defined by band edge potentials, component of SC demonstrates a vast effect on photo-catalytic performances. Particularly, for ultra-thin 2DMs, the electronic configuration strongly relies on equivalent constituent. Maeda et al. [91] formed $\mathrm{HCa}_{2-x} \mathrm{Sr}_{x} \mathrm{Nb}_{3} \mathrm{O}_{10}$ and $\mathrm{HCa}_{2} \mathrm{Nb}_{3-y} \mathrm{TayO}_{10} \mathrm{NSs}$ with restricted energy band structure via interlayer exchange $\mathrm{K}^{+}$-ions with protons in layered $\mathrm{KCa}_{2-x} \mathrm{Sr}_{x} \mathrm{Nb}_{3} \mathrm{O}_{10}$ as well as $\mathrm{KCa}_{2} \mathrm{Nb}_{3-y} \mathrm{Ta}_{y} \mathrm{O}_{10}$ and after that more undergo exfoliation. By gradually engineering atomic component in NSs, the optical absorption of materials is deeply suffered (Fig. 8).

Thoroughly, commencement of absorption edge in $\mathrm{HCa}_{2-x} \mathrm{Sr}_{x} \mathrm{Nb}_{3} \mathrm{O}_{10}$ showed a clear redshift with improved Sr-content, accompanying via BG energy reduced from 3.59 $(x=0)$ to $3.40 \mathrm{eV}(x=2)$. Additionally, onset of blueshift absorption edge was determined for $\mathrm{KCa}_{2} \mathrm{Nb}_{3-y} \mathrm{Ta}_{y} \mathrm{O}_{10}$ with higher Ta contents. The substitution of $\mathrm{Nb}^{5+}$ by $\mathrm{Ta}^{5+}$-ions caused more negative CB potential, which was liable for blueshift of the onset absorption edge. Thus, tunable light absorption performance was obtained by altering component and further showed an important effect on the photo-catalytic $\mathrm{H}_{2}$-evolution reaction. By utilizing an analogous plan, energy band structure of ternary sulfides, $\mathrm{H}_{2 x} \mathrm{Zn}_{1-x} \mathrm{In}_{2} \mathrm{~S}_{4}$, was efficiently engineered with $\mathrm{ZnIn}_{2} \mathrm{~S}_{4}$ and showed optimal photo-catalytic $\mathrm{H}_{2}$-evolution rate [138]. Another significant case was tuning of halogen $/ \mathrm{O}_{2}$ ratio in the bismuth oxy-halide to tune electronic structures. In accordance with DFT calculations, VB top of bi-oxy-halide was mainly composed of $\mathrm{O}_{2 p}$ and $\mathrm{X} n p(n=3,4$, and 5 for $\mathrm{X}=\mathrm{Cl}, \mathrm{Br}$, and $\mathrm{I})$ hybrid orbitals, while $\mathrm{CB}$ was primarily comprised of Bi6p orbitals. In the course of engineering $\mathrm{O}, \mathrm{X}$ elements, $\mathrm{BG}$ and band edge potentials were efficiently engineered. Thus far, a number of $\mathrm{Bi}$ oxy-halide NSs with tuned $\mathrm{O}, \mathrm{X}$ component were synthesized, for example $\mathrm{Bi}_{12} \mathrm{O}_{17} \mathrm{C}_{12}$ [139], $\mathrm{Bi}_{4} \mathrm{O}_{5} \mathrm{Br}_{2}$ [140], $\mathrm{Bi}_{4} \mathrm{O}_{5} \mathrm{I}_{2}$ [141], and so on. In general, with decreasing $\mathrm{Br}$ or $\mathrm{Cl}$ content, acquired materials showed narrowed $\mathrm{BG}$ than that of resultant $\mathrm{BiOCl}$ or $\mathrm{BiOBr}$ and reduction of $\mathrm{I}$ content resulted in an increase in $\mathrm{BG}$ comparative to $\mathrm{BiOI}$.
Benefiting from energy band structure range, tuned bi-oxyhalide ultra-thin NSs are showing superiority toward various photo-catalytic employments.

\subsection{Thickness Tuning}

SC thickness is an important factor to an electronic structure engineering and photo-catalytic performance optimization. As a result of known quantum confinement effect, BG of SC undergoes an increase, when materials thickness is reduced. Moreover, surface effect is aggravated as thickness reduced to an atomic size. The electronic DOSs can improve at the surface of ultra-thin 2DMs in comparison with interior of bulk materials. Such characteristics showed a significant impact on photo-catalytic efficiency of ultrathin 2DMs. Based on thermal oxidation etching way, ultrathin $\mathrm{C}_{3} \mathrm{~N}_{4} \mathrm{NSs}$ with thickness of $\sim 2 \mathrm{~nm}$ were formed [125]. As thickness reduced to atomic size, electronic structure of $\mathrm{C}_{3} \mathrm{~N}_{4}$ suffers from major difference. Consequently, due to quantum confinement effect, $\mathrm{BG}$ increased from $2.77 \mathrm{eV}$ for bulk $\mathrm{C}_{3} \mathrm{~N}_{4}$ to $2.97 \mathrm{eV}$ in ultra-thin 2D-NSs. Simultaneously, thickness reduction grants guarantee to improve an electron transport capability toward in-plane direction and enhanced duration of photo-generated charge carriers.

Additional research originates in which $\mathrm{CB}$ edge of ultrathin $\mathrm{C}_{3} \mathrm{~N}_{4}$ NSs showed upshift in comparison with bulk counterpart [142]. So, photo-generated $\mathrm{e}^{-} \mathrm{s}$ holds strong reduction capability and helps in improving the photo-catalytic $\mathrm{H}_{2}$-evolution performance. Despite $\mathrm{C}_{3} \mathrm{~N}_{4}$, electronic structure tuning is gained within other SCs through thickness engineering. Through thickness reduction of bulk $\mathrm{SnNb}_{2} \mathrm{O}_{6}$ to 50 and $3 \mathrm{~nm}$, resultant $\mathrm{BG}$ increased from 2.30 to 2.35 and $2.43 \mathrm{eV}$, respectively, causing upshifting of CB edge [96]. It is suggested that thickness engineering was an efficient way to alter energy band configuration of SCs. Moreover, when bulk materials thickness is regularly decreased to an atomic size thickness or even SL, the ratio of exposed surface atoms to whole atoms can be prominently improved. Lack of nearby atoms formed plentiful coordination on unsaturated surface atom with dangling bonds and is leaning to bond with other atoms to attain stability. So, these surface atoms displayed a high surface energy and chemical reactivity. The free-standing SnSe and SnS NSs with all exposure surface atoms were acquired through exfoliating their bulk counterparts in mixed solvent of $\mathrm{H}_{2} \mathrm{O}$ and ethanol [143]. Taking 

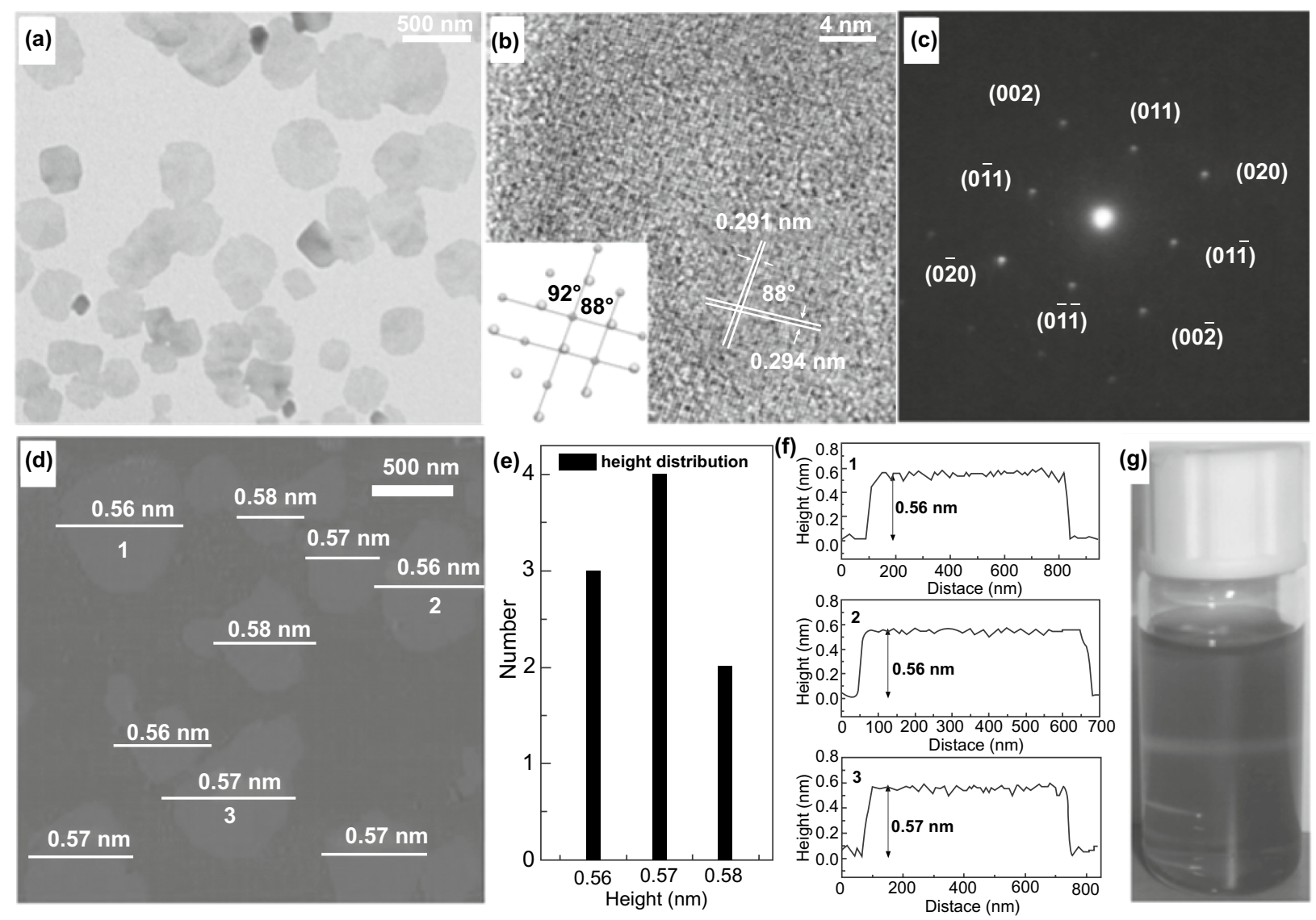

Fig. 9 a, b TEM and HR-TEM, c selected-area electron diffraction. d-f AFM image, height distribution, and height profiles. f (1-3) stands for 1-3 in d. g Colloidal $\mathrm{H}_{2} \mathrm{O}$ /ethanol (1:1) dispersion of the as-synthesized products showing Tyndall effect. Adapted with permission from Ref. [144]

$\mathrm{SnS}$ as an example, with large-area NSs resembling morphology with lateral size of almost $500 \mathrm{~nm}$, their transparent properties were studied by TEM investigation, showing ultra-thin thickness of as-synthesized material (Fig. 9). The average height of SnSe NSs, calculated by AFM, was about $0.57 \mathrm{~nm}$ that agrees with thickness of half unit cell. Hence, these SL-exfoliated NSs calculated band structures which proposed a change from an indirect SC in bulk $\mathrm{SnS}$ to direct SC for SnS SLs. In comparison with bulk SnSe, enhanced DOSs were obtained at VB edge of SL SnS, which were enabling SL SnS with improved carrier transport efficiency. To get benefit from SL structure, SLs SnS showed improved photo-absorption and charge separation efficiency and later supported $\mathrm{H}_{2} \mathrm{O}$ splitting performance.

\subsection{Doping}

The appropriate doping was observed as a competent approach for engineering physicochemical property of 2DMs. Conscious manufacturing of extrinsic metal or nonmetal species into SC lattice presents the prospect to adjust electronic or surface configurations of host material for enhancing photo-catalytic performance. Types and allocation of dopants are very important to control properties of host SCs. Advantages of atomic size of 2DMs; doping perhaps is a very sufficient plan to influence properties of ultra-thin 2DMs. Normally, doping always happens on bulk materials' shallow surface due to lack of atoms accessing gallery that demonstrated a small manipulation 

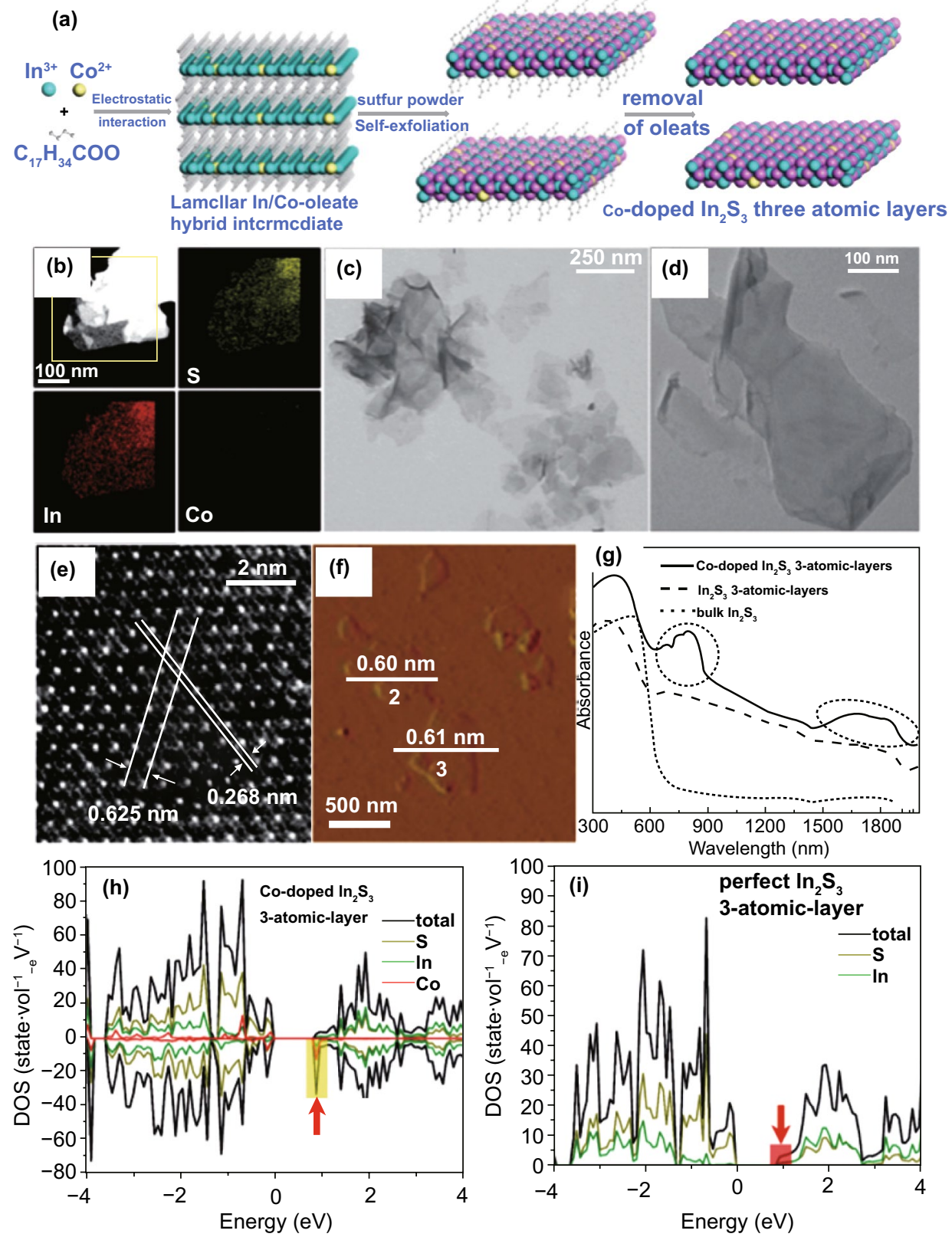

Fig. 10 a Representation of the formation of Co-doped $\operatorname{In}_{2} S_{3}$ three atomic layers. b-g Characterizations for Co-doped $\operatorname{In}_{2} S_{3}$ three atomic layers: b HAADF-STEM image and EDS mapping of an individual Co-doped $\operatorname{In}_{2} \mathrm{~S}_{3}$ three atomic layer, c-e TEM images and HR-TEM image, $\mathbf{f}$, $\mathbf{g}$ AFM image, and height profiles. $\mathbf{h}$ DOSs of Co-doped $\operatorname{In}_{2} \mathrm{~S}_{3}$ three atomic layer slabs and $\mathbf{i}$ ideal $\operatorname{In}_{2} \mathrm{~S}_{3}$ three atomic level slab. Adapted with permission from Ref. [147]

on their total performance. Concerning ultra-thin 2DMs, atomic thickness permits efficient doping of dopants and just needs small diffusion penetration. So, it is enviable to tailor heteroatom into 2DMs and builds high competence methods.

\subsubsection{Metal Doping}

Metal ions incorporation into crystal lattice causes a rise in impurity levels in SCs forbidden band. Onset light absorption edge redshift is frequently examined that is recognized to cause 
a transition of impurity quantities to CB or VB. Normally, the very capable doped photo-catalysts primarily depend on doping alteration of metal ions that satisfied the criteria, i.e.,

1. The $\mathrm{e}^{-} \mathrm{s}$ and $\mathrm{h}^{+} \mathrm{s}$ can be trapped through dopant and make sure efficient confined separation.

2. The captured $\mathrm{e}^{-} \mathrm{s}$ and $\mathrm{h}^{+} \mathrm{s}$ are generated and transferred on surface successfully.

Xie and co-authors [145] doped $\operatorname{In}_{2} \mathrm{~S}_{3}$ NSs with Co to optimize photo-catalytic $\mathrm{H}_{2} \mathrm{O}$ splitting. Through a lamellar inorganic-organic hybrid intermediate approach, Co-doped $\mathrm{In}_{2} \mathrm{~S}_{3}$ NSs ( $0.59 \mathrm{~nm}$ thick) along with 3-atomic layers thickness were formed (Fig. 10). The electronic configuration of $\operatorname{In}_{2} \mathrm{~S}_{3}$ using three atomic layers and Co-doped $\operatorname{In}_{2} \mathrm{~S}_{3}$ was first verified via DFT simulations. To simulate existence of Co-dopant, certain ultra-thin $\operatorname{In}_{2} \mathrm{~S}_{3}$ NSs with noticeably enhanced DOS are developed at conduction band maximum (CBM) as compared with perfect, ultra-thin $\operatorname{In}_{2} \mathrm{~S}_{3}$ NSs. Additionally, Co-ion doping provides a Co-doped $\mathrm{In}_{2} \mathrm{~S}_{3}$ material with numerous other energy levels that were resultant from $\mathrm{Co}_{3 \mathrm{~d}}$ splitting. Under light irradiation, $\mathrm{e}^{-} \mathrm{s}$ was simply excited through $d \rightarrow d$ internal transition of the Co-ions in tetrahedral coordination, allowing generation of more photo-generated $\left(\mathrm{e}^{-}-\mathrm{h}^{+}\right)$-pairs. These results were confirmed through light absorption difference of $\operatorname{In}_{2} \mathrm{~S}_{3}$ NSs and Co-doped $\operatorname{In}_{2} \mathrm{~S}_{3}$ in (UV-Vis)-diffusion reflectance spectrum. Considerable advancement in light absorption was viewed from 600 to $2000 \mathrm{~nm}$ that was consigned to creation of dopant energy levels of Co. To get advantages from doping of Co, photo-generated charge separation effectiveness enhanced about 25 -fold increase in average recovery duration, as practiced through an ultrafast transient absorption spectroscopy (UTAS). Therefore, Co-doping permits 10 times developed photo-catalytic activity for $\mathrm{H}_{2} \mathrm{O}$ splitting compared to perfect $\operatorname{In}_{2} \mathrm{~S}_{3}$ NSs. Similarly, other metal elements were also utilized for doping to engineer electronic structure of ultra-thin 2D-hosted photo-catalyst, for instance $\mathrm{Pt}, \mathrm{Rh}, \mathrm{Cr}, \mathrm{Fe}, \mathrm{Cu}$, and so on [146]. For example, $\mathrm{Fe}$ was doped into ultra-thin BiOCl NSs, which extended light absorption range from UV to Vis-light. Photo-catalytic activity for pollutant removal and $\mathrm{H}_{2}$-evolution was increased. These results certainly verified that metal element doping is an efficient way to tune electronic structure of ultra-thin $2 \mathrm{D}$ photo-catalysts and can promote photocatalytic performance.

\subsubsection{Nonmetal Doping}

Concerning nonmetal elements doping, two direct theories were suggested to modify an electronic arrangement and hence influence photo-catalytic performance. First, dopants can generate localized states between forbidden bands and one advantage is fusing of dopant-occupied positions with VB and upshifting of valence band maximum (VBM). Such two diverse techniques normally originate from different doping types, where surface doping will cause development of localized states and uniform doping will promote VBM [148]. As mobility of $\mathrm{h}^{+} \mathrm{s}$ in localized states is slow and after that restricts photo-catalytic efficiency, offering uniform allocation of dopant to upshift VBM and encourage $\mathrm{h}^{+} \mathrm{s}$ relocation is preferred much. The atomic thickness of the 2DMs facilitated a uniform doping because little doping depth is specifically needed [149]. By tailoring $\mathrm{O}_{2}$-atoms for $2 \mathrm{D} \mathrm{ZnIn}_{2} \mathrm{~S}_{4} \mathrm{NSs}$ to replace sulfur atoms lattice, electronic configuration suffered by diverse differences from the pristine $\mathrm{ZnIn}_{2} \mathrm{~S}_{4}$ NSs [150]. So, the DFT-based calculations showed that $\mathrm{O}_{2}$-doping effectively reinforces DOS at VBM versus pristine $\mathrm{ZnIn}_{2} \mathrm{~S}_{4}$, enlightening creation of enhanced charge density around VBM. Both of CB and VB edge in O-doped $\mathrm{ZnIn}_{2} \mathrm{~S}_{4}$ showed the upshift concerning $\mathrm{ZnIn}_{2} \mathrm{~S}_{4} \mathrm{NSs}$, as verified through UV-Vis absorption spectrum and XPS VB spectrum. It enhanced CBM as well as improved the VB distance across obtained with very superior mobility and improved the expenditure of photo-generated $\mathrm{h}^{+} \mathrm{s}$, thus to support $\mathrm{H}_{2}$ production. Wang and co-authors studied that $\mathrm{C}$-atoms doping can efficiently refrain electronic configuration of h-BN (Fig. 11) [151]. So, the DFT simulation showed that $\mathrm{BG}$ of h-BN was obtained to be $4.56 \mathrm{eV}$. After C-doping in structure, $\mathrm{BG}$ was notably reduced. Regarding $\mathrm{B}_{11} \mathrm{C}_{12} \mathrm{~N}_{9}$ compound, $\mathrm{BG}$ was narrowed to $2.00 \mathrm{eV}$, through $\mathrm{VB}$ and $\mathrm{CB}$ edges which mostly consist of $\mathrm{C}_{2 p}$ orbitals. $\mathrm{B}_{11} \mathrm{C}_{12} \mathrm{~N}_{9}$ VB top states were not localized in comparison with pure $\mathrm{BN}$. Taking advantage of C-doping with $s p^{2}$-delocalization system, ultra-thin C-BN NSs with thickness of almost 3-4 nm were achieved and displayed an outstanding vislight photo-catalytic performance to evolution of $\mathrm{H}_{2}$ and reduction of $\mathrm{CO}_{2}$. Considering insulator characteristic of pristine h-BN, it is illustrated that nonmetal doping can endorse photo-catalytic performance and also create promising photo-catalytic performance. 
(a)

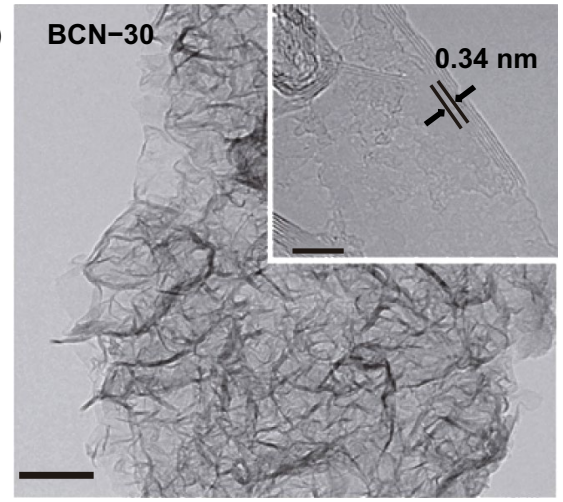

(b)

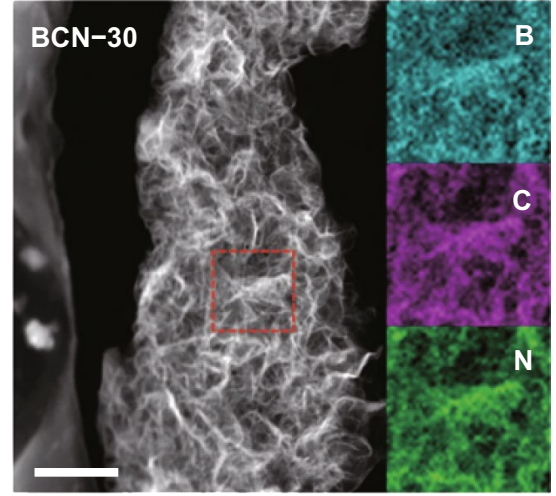

(c)

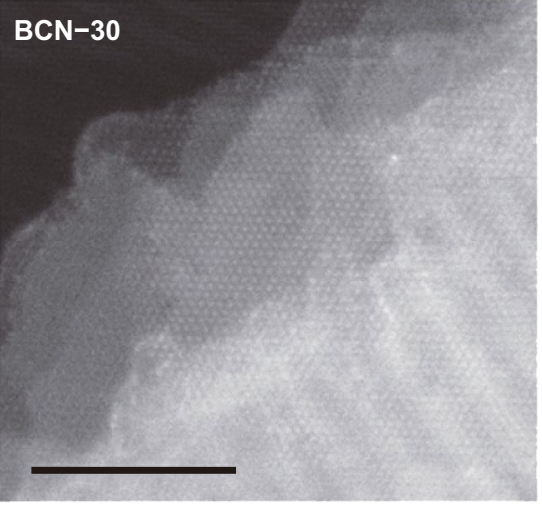

(d)
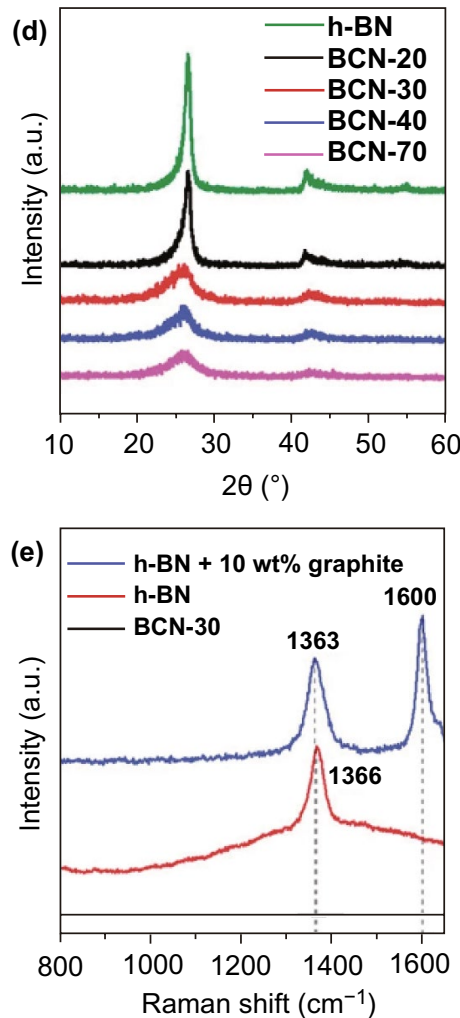

(f)

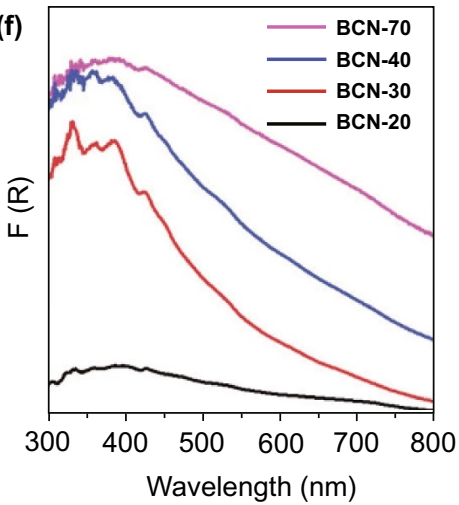

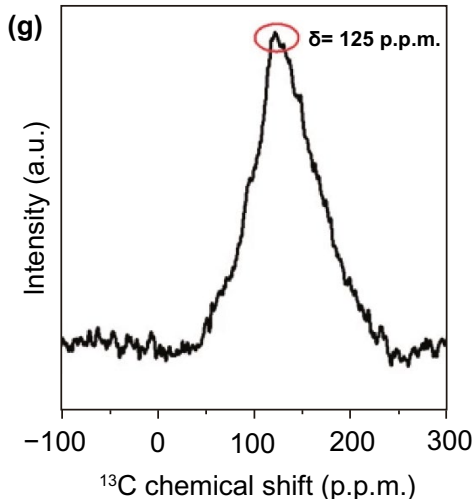

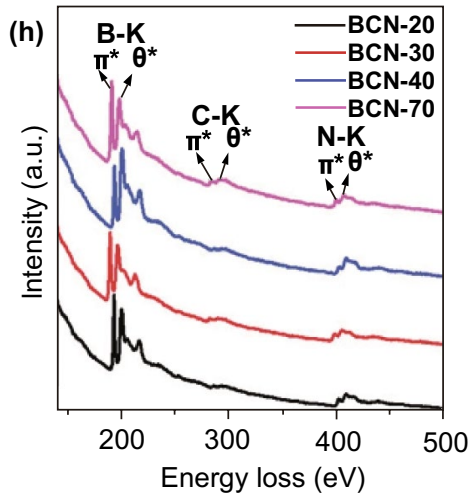

(i)

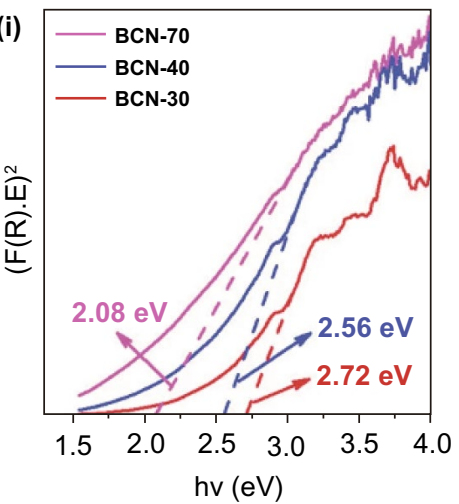

Fig. 11 a HR-TEM of BCN-30. b Dark-field TEM image of BCN-30 and element map. c HR-STEM of the BCN-30 sample along (002) facet. d XRD of h-BCN. g NMR spectra of BCN-30. e Raman spectrum of h-BN and BCN-30 and physical mixture of h-BN and graphite. $\mathbf{h}$ EELS spectrum of BCN- $x$. f UV-Vis diffuse reflectance spectra of BCN- $x$. i BG calculation of BCN- $x$ from the (F(R) E)n versus E plots. Adapted with permission from Ref. [151]

\subsection{Defect Engineering}

Aside from doping, defect engineering also demonstrates an important effect on ultra-thin 2DMs, in case of photo-catalysis. Owing to 2D atomic size thin structure, in the presence of defects, it has strong influence on fundamental properties, in spite of a very low-level doping. In relation to huge surface defects formation in the bulk materials, ultra-thin 2DMsNSs with relatively small atomic escape energy can propose an important chance to get a range of defects. So, it is necessary to construct surface defects, for instance anion, cation vacancies, pits, vacancy association, and distortions, to efficiently optimize electronic configuration of ultra-thin 2D photo-catalysts. 
(a)

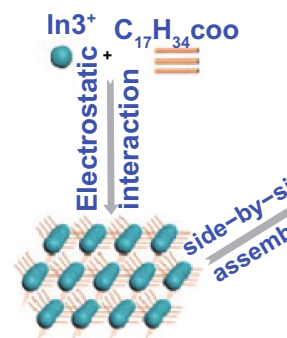

Hexagonal mesostructured In-oleate complex

(b)

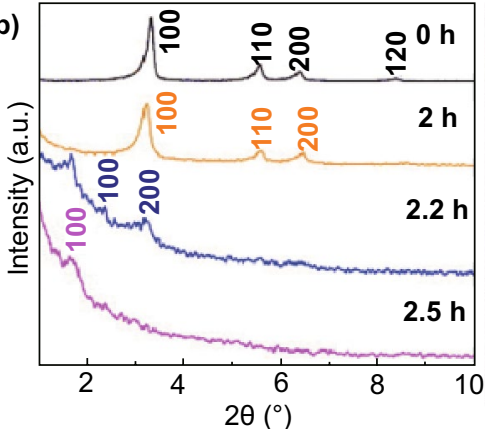

(e)

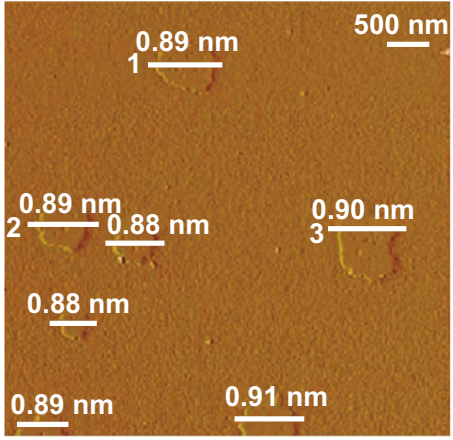

$\mathrm{V}_{0}$-rich ultrathin

$\ln _{2} \mathrm{O}_{3}$ porous sheets

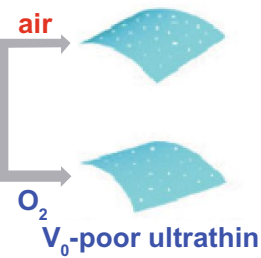

Ultrathin In $(\mathrm{OH})_{3}$ sheets
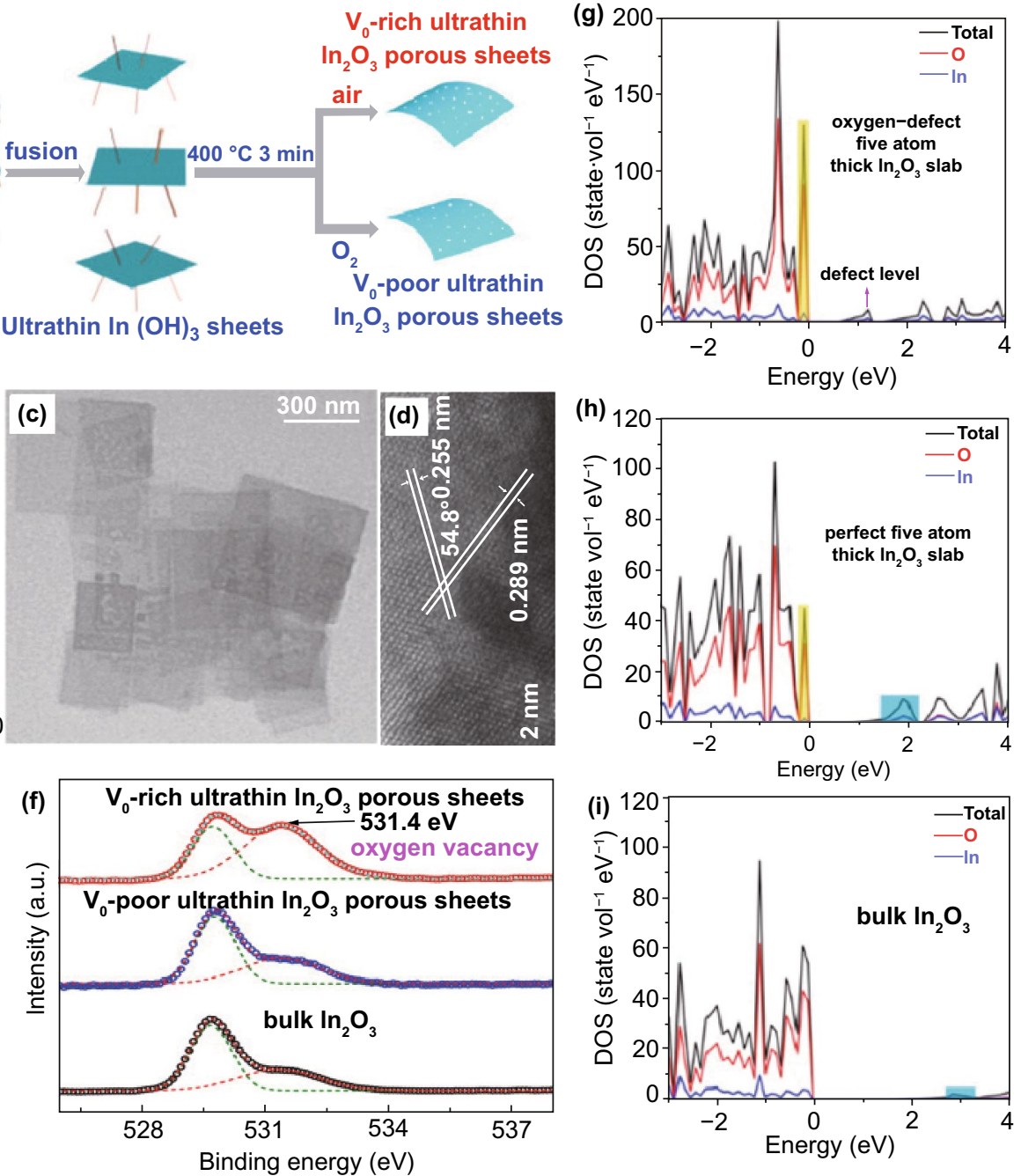

Fig. 12 a Scheme showing the development of VO-rich/VO-poor atomically thin $\mathrm{In}_{2} \mathrm{O}_{3}$ porous NSs under special environment. b Timedependent small-angle XRD patterns for the as-synthesized precursors. c-f Characterizations for the VO-rich atomically thin $\mathrm{In}_{2} \mathrm{O}_{3}$ porous $\mathrm{NSs}$ obtained via rapid thermal heat treatment of $\mathrm{In}(\mathrm{OH})_{3}$ NSs in air. c, $\mathbf{d}$ TEM/HR-TEM image. e AFM analysis. f O 1s XPS spectra. g Electron spins resonance spectrum. h Simulated DOS of $\mathrm{O}_{2}$ defect five-atom-thickness $\operatorname{In}_{2} \mathrm{O}_{3}$ slab. i ideal five-atom-thickness $\mathrm{In}_{2} \mathrm{O}_{3}$ slab. Adapted with permission from Ref. [84]

\subsubsection{Anion Vacancies}

In anion vacancy type, $\mathrm{V}_{\mathrm{O}}$ was broadly studied due to its small creation energy and prevalence in the oxide materials [152]. For example, Fengcai Lei et al. [84] studied that by fast heating of intermediate $\mathrm{In}(\mathrm{OH})_{3} \mathrm{NSs}$ in $\mathrm{O}_{2}$ or air, $\mathrm{V}_{\mathrm{o}}$-rich and $\mathrm{V}_{\mathrm{o}}$-deficient $\mathrm{In}_{2} \mathrm{O}_{3} \mathrm{NSs}$ were formed in fully controlled way, respectively. Figure 12 shows the AFM image, which showed thickness of $\operatorname{In}_{2} \mathrm{O}_{3}$ NSs to be almost $0.9 \mathrm{~nm}$, enlightening controlled formation of $\mathrm{In}_{2} \mathrm{O}_{3}$ materials with atomic thickness. ESR and XPS spectrum results showed the presence of $\mathrm{V}_{\mathrm{o}}$. The observed $531.4 \mathrm{eV}$ peak showed $\mathrm{V}_{\mathrm{o}}$-rich ultra-thin $\mathrm{In}_{2} \mathrm{O}_{3} \mathrm{NSs}$, which have maximum peak area, signifying that more $\mathrm{V}_{\mathrm{O}}$-rich ultra-thin $\mathrm{In}_{2} \mathrm{O}_{3}$ NSs were formed compared to $\mathrm{V}_{\mathrm{o}}$-poor ultra-thin $\operatorname{In}_{2} \mathrm{O}_{3}$ NSs and bulk counterpart. Moreover, sharp $\mathrm{V}_{\mathrm{o}}$ signal at $g=2.004$ in ESR spectrum was also observed that shows $\mathrm{V}_{\mathrm{o}}$-rich $\mathrm{In}_{2} \mathrm{O}_{3} \mathrm{NS}$ sample holds the highest level of $\mathrm{V}_{\mathrm{o}}$. In $\mathrm{V}_{\mathrm{o}}$ engineering, electronic configuration of $\mathrm{In}_{2} \mathrm{O}_{3} \mathrm{NSs}$ with rich $\mathrm{V}_{\mathrm{o}}$ will experience noticeable change. As authorized 


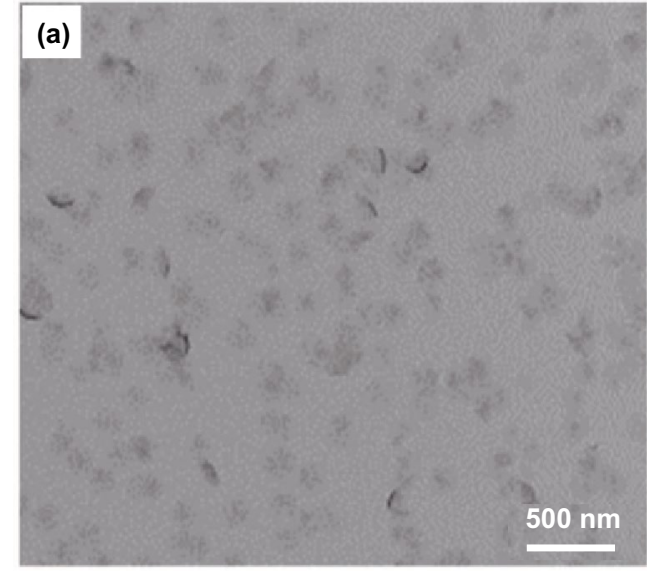

(c)
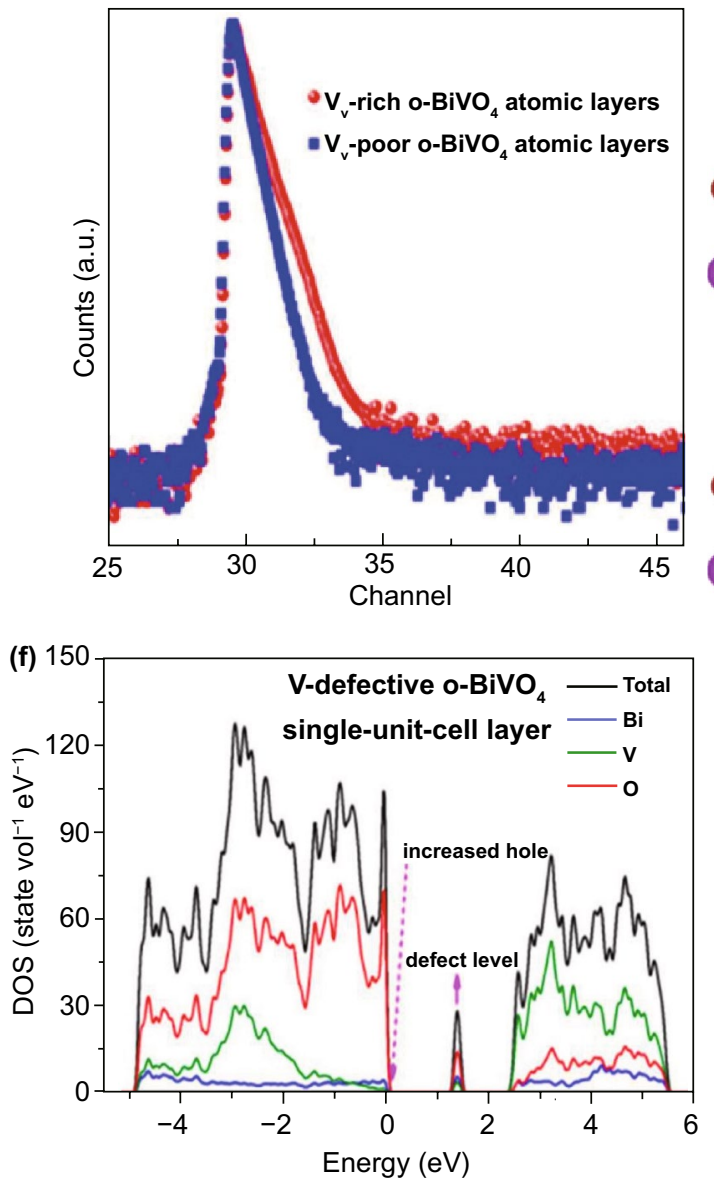

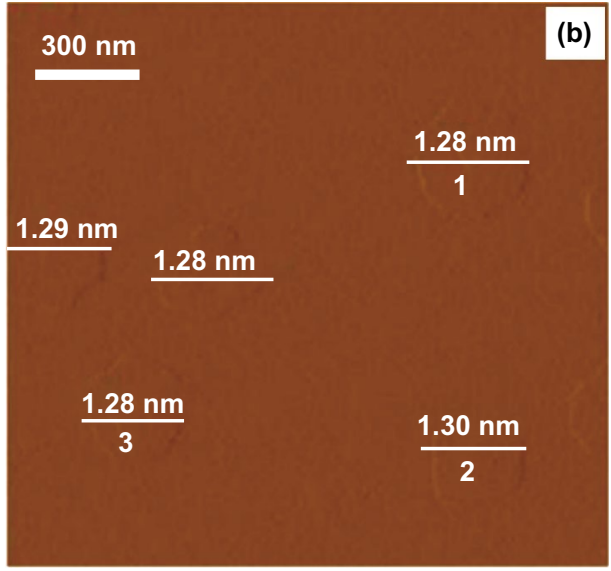

(e)
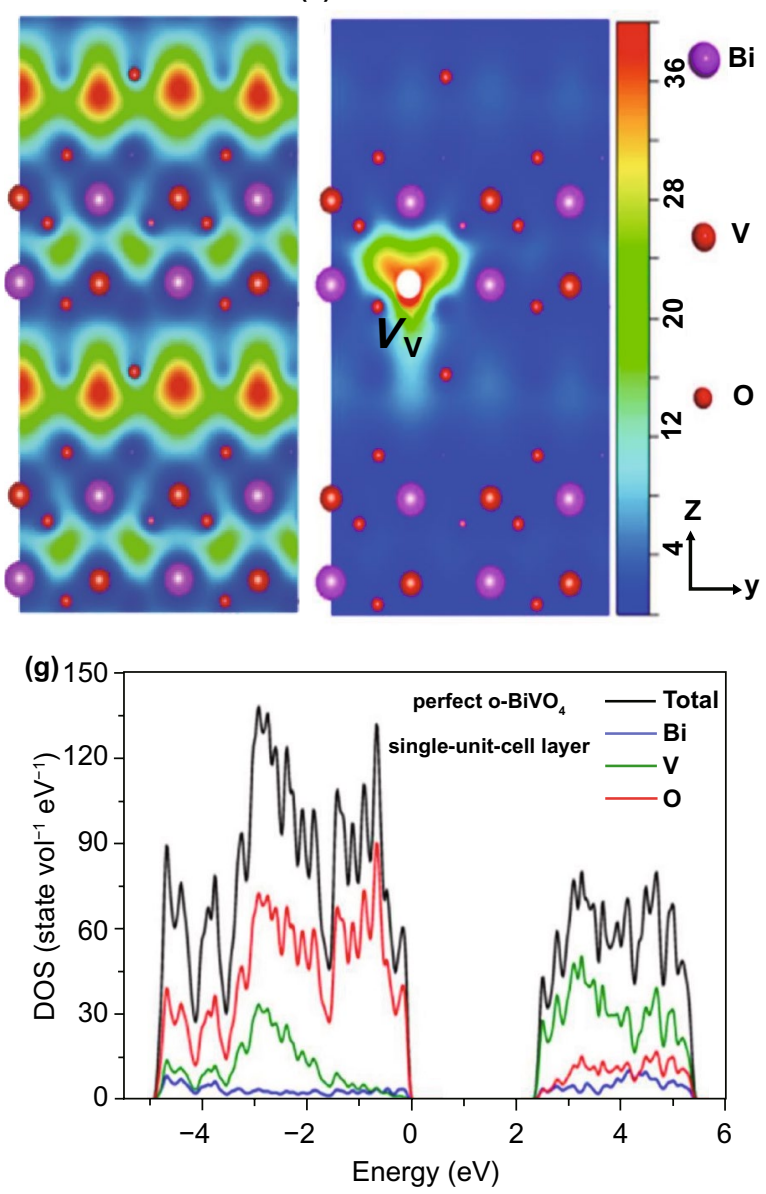

Fig. 13 a, b TEM and AFM analysis of one-unit-cell thickness $\mathrm{V}_{\text {v-rich o-BiVO }}$. C-e Defects study of $\mathrm{V}_{\mathrm{v}}$-rich and $\mathrm{V}_{\mathrm{v}}$-poor o-BiVO $\mathrm{P}_{4}$ atomic layers. $\mathbf{c}$ Positron duration spectra. d, e Scheme of entrapped positrons. f, $\mathbf{g}$ DOSs calculation of the $\mathrm{V}$ defects in o-BiVO ${ }_{4}$ single-unit-cell layer slab and pure o- $\mathrm{BiVO}_{4}$ single-unit-cell layer slab (f), along [001] direction (g). Adapted with permission from Ref. [154]

through DRS analysis and the XPS VB, spectrum, $\mathrm{V}_{\mathrm{o}}$-rich $\mathrm{In}_{2} \mathrm{O}_{3}$ NSs showed a narrowed BG and upshifted VB edge. DFT simulations clearly show that enhanced DOS at VBM was created and a novel defect concentration showed $\mathrm{V}_{\mathrm{o}}$-rich $\mathrm{In}_{2} \mathrm{O}_{3}$ than $\mathrm{V}_{\mathrm{o}}$-poor $\mathrm{In}_{2} \mathrm{O}_{3}$ NSs. So, the $\mathrm{V}_{\mathrm{o}}$-rich $\operatorname{In}_{2} \mathrm{O}_{3} \mathrm{NSs}$ acquired higher carrier level and enhanced electric field in 
space charge regions. The $\mathrm{e}^{-} \mathrm{s}$ were further simply excited into $\mathrm{CB}$ in irradiation. Therefore, $\mathrm{V}_{\mathrm{o}}$-rich $\mathrm{In}_{2} \mathrm{O}_{3} \mathrm{NSs}$ showed 2.5 and 15 times enhanced photo-catalytic performance as compared to $\mathrm{V}_{\mathrm{o}}$-poor $\mathrm{In}_{2} \mathrm{O}_{3} \mathrm{NSs}$ as well as bulk $\mathrm{In}_{2} \mathrm{O}_{3}$, correspondingly, for $\mathrm{H}_{2} \mathrm{O}$ oxidation. Such outcomes certainly verified the efficient role of anion vacancy in electronic configuration engineering.

(a)

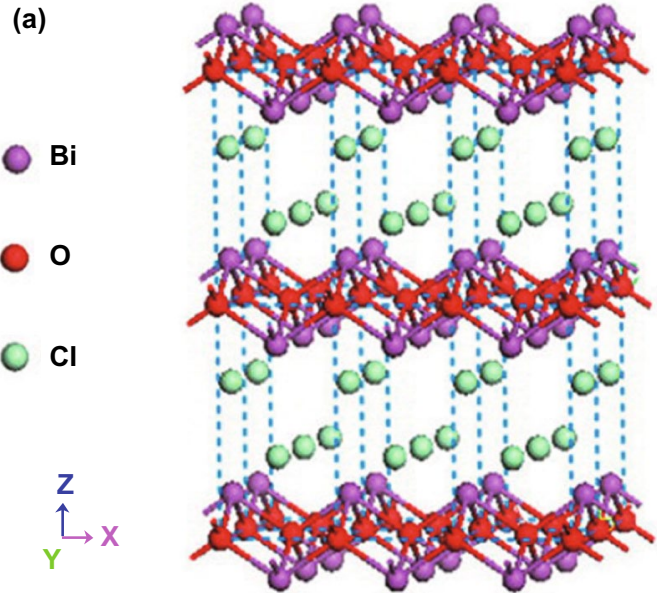

\subsubsection{Cation Vacancies}

Besides anion, cation vacancies are extremely efficient approach to cause useful electronic structure modification of ultra-thin nano-structure due to multifarious electron arrangement and orbit. For example, vanadium (V) vacancies $\left(V_{v}\right)$ were initiated in single-unit-cell $\mathrm{BiVO}_{4}$

(b)
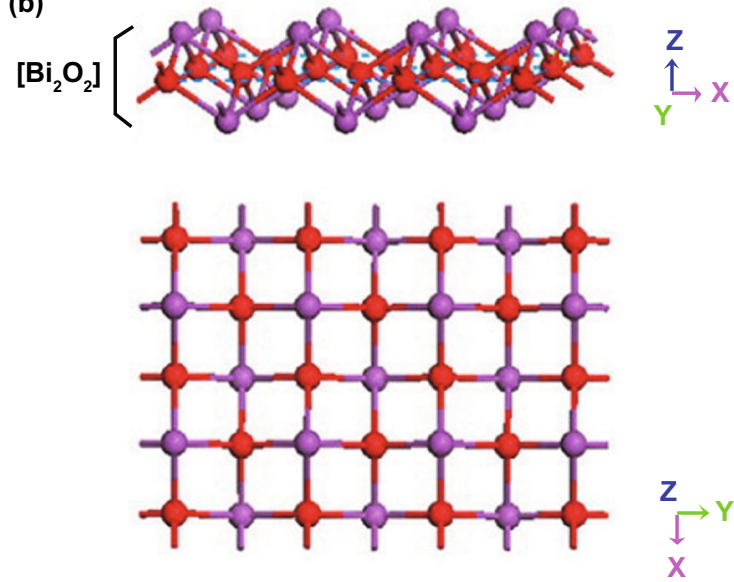

(c)

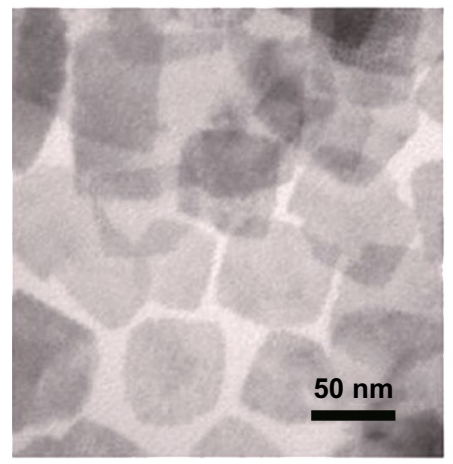

(e)

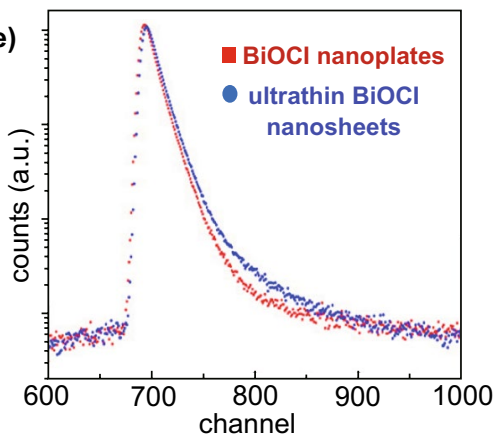

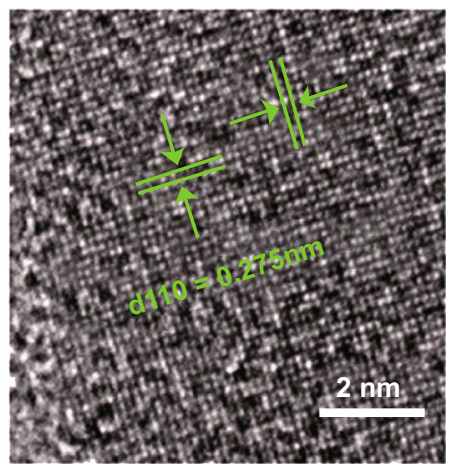

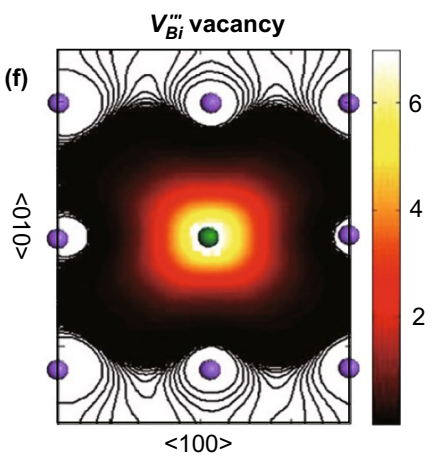

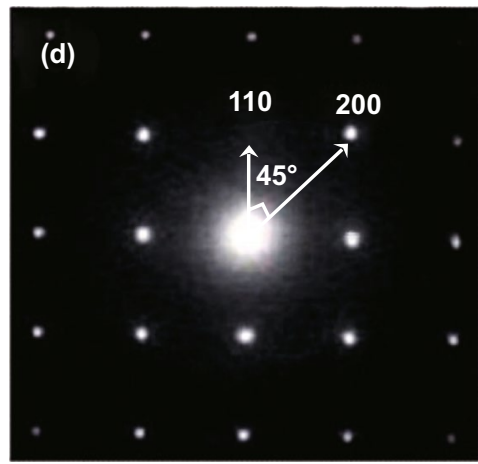

$V_{B i}^{\prime \prime \prime} V_{O}^{\prime \prime} V_{B i}^{\prime \prime \prime}$ vacancy associates

(g)

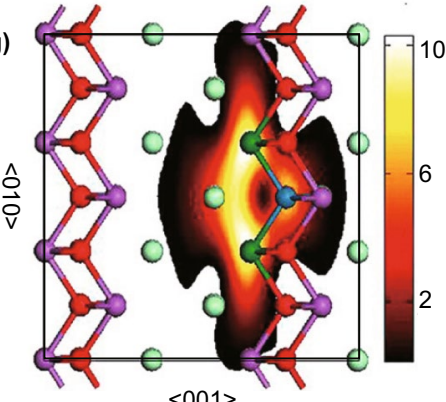

Fig. 14 Scheme showing the crystal structure of $\mathrm{BiOCl}$. a 3D-projection. b, $\mathbf{c}\left[\mathrm{Bi}_{2} \mathrm{O}_{2}\right]^{2+}$ layers along with the [010] and [001] directions, correspondingly. d TEM/HR-TEM of BiOCl NSs. e Positron lifetime spectra of ultra-thin BiOCl NSs and BiOCl NPs. f, g Scheme showing trapped positrons of $\mathrm{VBi}^{\prime \prime \prime}$ defect and $\mathrm{VBi}^{\prime \prime \prime} \mathrm{VO} \mathrm{VBi}^{\prime \prime}$-associated vacancy, correspondingly. Adapted with permission from Ref. [119] 
NSs (1.28 nm) along diverse quantities (Fig. 13) [153]. The atomic level concentration of the $\mathrm{V}_{\mathrm{v}}$ was done through positron annihilation spectrometry (PAS) and X-ray fluorescence (XRF). For $\mathrm{BiVO}_{4}$ the shortest life component $\left(\tau_{1}\right.$, around $200 \mathrm{ps}$ ) approved from PAS that trapped at $\mathrm{V}_{\mathrm{v}}$, helpful for subsistence of $\mathrm{V}_{\mathrm{v}}$ for two samples. Comparative positron intensity duration for $\mathrm{V}_{\mathrm{v}}$-rich $\mathrm{BiVO}_{4} \mathrm{NSs}$ was denoted for higher $\mathrm{V}_{\mathrm{v}}$ level. Furthermore, elemental ratio of $t \mathrm{~V}$ and $\mathrm{Bi}$ was verified to be 0.914 and 0.976 in $\mathrm{V}_{\mathrm{v}}$-rich $\mathrm{BiVO}_{4} \mathrm{NSs}$ and $\mathrm{V}_{\mathrm{v}}$-poor $\mathrm{BiVO}_{4} \mathrm{NSs}$, in that order proposing concentration difference of $\mathrm{V}_{\mathrm{v}}$. Benefiting from incidence of $\mathrm{V}_{\mathrm{v}}$, a novel defect level can be produced in BG of $\mathrm{BiVO}_{4}$, as confirmed via DFT simulation, which leads $\mathrm{e}^{-} \mathrm{s}$ further capable to be excited into C.B. Additionally, higher DOS at VB edge was achieved because of $V_{v}$ engineering. So, engineered vacancies increased the light harvest and promoted electronic conductivity for $\mathrm{V}_{\mathrm{v}}$-rich $\mathrm{BiVO}_{4}$. Simultaneously, abundant $\mathrm{V}_{\mathrm{v}}$ allowed an efficient charge separation that prolonged carriers' lifetime from 74.5 to 143.6 ns. Taking advantages from such $\mathrm{V}_{\mathrm{v}}$, an advanced photo-catalytic performance was attained from methanol synthesis rate up to $398.3 \mu \mathrm{mol} \mathrm{g}^{-1} \mathrm{~h}^{-1}$.

\subsubsection{Associated Vacancies}

The lost surface atoms not just introduced mono-vacancy but also vacancies associated were able to appear. As a result of multi-atomic vacancy coupling, vacancy associated can strongly engineer electronic structure and cause amazing electronic performance. For instance, triple vacancy of VBi"'VO"VBi"' were built in ultra-thin BiOCl NSs (thickness $=2.7 \mathrm{~nm}$ ) with dimension engineering (Fig. 14) [119]. Generated associated triple vacancy $\mathrm{VBi}^{\prime \prime \prime} \mathrm{VO} \mathrm{VBi}^{\prime \prime \prime}$ was verified through PAS. When Bi-atoms' outer surface was exposed in $\mathrm{BiOCl}$ crystal configuration, it is very likely to break out from lattice to make vacancy. While thickness was decreased to an atomic size, $\mathrm{O}_{2}$-atoms that linked to $\mathrm{Bi}$ atoms in an internal layer also escaped more effortlessly. So, control defects in $\mathrm{BiOCl}$ nano-plates were separated $\mathrm{VBi}^{\prime \prime \prime}$, whereas that changed its associated vacancy $\mathrm{VBi}^{\prime \prime} \mathrm{VO} \mathrm{VBB}^{\prime \prime \prime}$ in ultra-thin BiOCl NSs. Different defect types certainly affect the electronic structure that guarantees BiOCl NSs with enhanced adsorption of RhB molecules due to further negative charge. Benefiting from defect types changing from VBi"' to VBi"'VO"VBi"', ultra-thin BiOCl NSs showed both upshifted CB and VB potentials that favor charge mobility and therefore allowed enhanced separation of $\left(\mathrm{e}^{-}-\mathrm{h}^{+}\right)$-pairs. Therefore, ultra-thin BiOCl NSs showed great solar photocatalytic activity toward removal of pollutants.

\subsubsection{Distortion}

Besides the vacancies, other defects, for instance, distortions and pits were also sufficient policies for changing electronic structure of the ultra-thin 2D photo-catalysts. For instance, O'Hare and his research team [99] formed NiTi-LDH NSs through controlled thickness during reverse micro-emulsion approach. As obtained NiTi-LDH NSs, local atomic arrangement was examined through the X-ray absorption near-edge spectroscopy (XANES). While thickness significantly decreased, the titanium cation $\left(\mathrm{Ti}^{4+}\right)$ with lowered oxidation state was achieved in NiTi-LDH NSs $(\approx 2 \mathrm{~nm}$ thickness $)$, whereas it is approximately completely $\mathrm{Ti}^{4+}$ in bulk NiTiLDH. In comparison with bulk NiTi-LDH, lower coordination numbers of Ti-cations were seeing in NiTi-LDH NSs and experience severs crystal deformations. Consequently, NiTi-LDH NSs showed distinguishing electronic crystal lattice and enhanced $\mathrm{e}^{-}$transfer effectiveness comparative to bulk NiTi-LDH.

\subsubsection{Pits}

Pits formation gives a good technique to enhance surface defects which produced more coordinated unsaturated atoms with dangling bonds around pits in NSs. Through thermal treatment of bulk g- $\mathrm{C}_{3} \mathrm{~N}_{4}$ inNH $_{3}$ environment, NSs are efficiently exfoliated and many in-plane pits were built in $\mathrm{g}_{-} \mathrm{C}_{3} \mathrm{~N}_{4} \mathrm{NSs}$ [124]. In-plane pits formation damaged $\mathrm{g}-\mathrm{C}_{3} \mathrm{~N}_{4}$ plane structure and supplies promising active sites with dangling bonds. These dangling bonds serve as cross-plane diffusion pathways to speed up mass transfer and charge diffusion. Furthermore, formed pits are promoted as a result in creation of $\mathrm{C}$-vacancies owing to unbalance structure. So, g- $\mathrm{C}_{3} \mathrm{~N}_{4}$ NSs showed improved $\mathrm{BG}$ and absolute light absorption area matching with bulk counterpart. Simultaneously, pit-rich g- $\mathrm{C}_{3} \mathrm{~N}_{4} \mathrm{NSs}$ guarantee superior $\mathrm{CB}$ and $\mathrm{VB}$ potential and give advanced $\mathrm{e}^{-}$donor density. Thus, 20 times high photo-catalytic $\mathrm{H}_{2}$-evolution activity was attained in vis-light irradiation. The aforementioned investigation results give a new and 
deep insight to comprehend method of action of surface defects in supporting photo-catalytic activity from energy band structure, surface charge, and SASs. Usually, as relative to defect poor counterpart, engineered defects in ultrathin 2DMs can alter electronic configuration, generally with improved DOS at edge of VB or CB, or even create a novel defect concentration in between forbidden band. Generally, light absorption possibility of genuine materials with redshift and hence light harvesting capability can be improved. At surface, limited charge density can also be altered and might make possible adsorption and activation of the target molecules. Simultaneously, formed surface defects can develop carrier level in the photo-catalysts and provide charge separation centers to entrap the carriers, encouraging consumption effectiveness of $\mathrm{h}^{+} \mathrm{s}$ and $\mathrm{e}^{-} \mathrm{s}$ for analogous interfacial redox responses.

\subsection{Anisotropic Effects in Catalysis}

Photo-catalytic routes could show vital to sustainable manufacturing of fuels and chemicals necessary for carbon-neutral society. An $\left(\mathrm{e}^{-}-\mathrm{h}^{+}\right)$recombination is a serious issue that has till date limited efficiency majority of potential photo-catalysts. Therefore, Matteo Cargnello et al. [155] showed the efficiency of anisotropy in enhancing charge separation and thus increasing activity of $\mathrm{TiO}_{2}$ photo-catalytic method. Particularly, they showed that $\mathrm{H}_{2}$ fabrication in homogeneous, $1 \mathrm{D}$ brookite- $\mathrm{TiO}_{2}$ nano-rods was highly improved through engineering their length. Utilizing respective characterization techniques to separately investigate excited $\mathrm{e}^{-} \mathrm{s}$ and $\mathrm{h}^{+} \mathrm{s}$, linked high observed reaction rates to anisotropic arrangement support competent carrier use. The QY for $\mathrm{H}_{2}$-fabrication from $\mathrm{C}_{2} \mathrm{H}_{5} \mathrm{OH}$, $\mathrm{C}_{3} \mathrm{H}_{8} \mathrm{O}_{3}$, and $\mathrm{C}_{6} \mathrm{H}_{12} \mathrm{O}_{6}$ as high as $65 \%, 35 \%$, and $6 \%$, correspondingly, showed generalization of this method for enhancing the photo-activity of SC-NMs for a broad range of reaction systems.

\section{Hybridization}

Unlike bulk materials, 2DMs have become a hot topic in academic field due to their atomic layer thickness, broadband absorption, and ultrafast optical response, which have been widely applied in the ultrafast laser generation [156-183], optical switching and modulators [184-197], optoelectronics devices [183, 198-203], and biosensor and biotherapy [14, 44, 204-214]. 2DMs have an ultra-large SSA to make sure that surface state is even more significant compared to bulk inside. Photo-generated charge carriers will be distributed at surface to engage in the redox reactions, to be discussed next. So, surface hybridization to present size element to support consumption effectiveness of charge carriers is enviable below precondition of 2D arrangement. Consistent with surface hybridization, numerous representative hybridizations with 2D structure are introduced, e.g., QDs/2DMs, single atoms/2DMs, molecular/2DMs, and 2D-2D stacked materials.

\subsection{Quantum Dots/2DMs Hybridization}

Coordinated unsaturated surface atoms of NPs have dangling bonds that used energy. To further decrease NPs' size, bigger part of surface atoms compared with total atoms will be attained and their regular atom binding energy must be high. So, if the size of NPs can control the QDs and modify 2DMs, interfacial strong coupling among them can be manufactured. Furthermore, QDs can show high dispersion on 2DMs because of their small size; those probably effective co-catalysts furthermore enhance the photo-catalytic activity. To develop operation effectiveness of $\mathrm{Ag}, \mathrm{Ag}$-QDs with size $>5 \mathrm{~nm}$ were formed. After hybridization with $\mathrm{BiOBr}$ NSs, photo-catalytic activity has been significantly boosted to degrade tetracycline hydrochloride, ciprofloxacin (CIP), and rhodamine B after vis-light irradiation. It was studied that tailored AgQDs activate molecular $\mathrm{O}_{2}$ through hot $\mathrm{e}^{-}$which decrease after vis-light exposure. The Ag-QDs can concurrently provide charge separation centers, adsorption centers, and photo-catalytic reaction centers, which are dependable on enhanced photo-catalytic efficiency. To decrease service of noble metals, non-noble metals or even metals-free QDs are taken as other choice. Also, thickness of subjected 2DMs can be further decreased to SL, in order to get improved dispersion and interfacial contact. For example, nitrogen-doped carbon QDs (N-CQDs) through $3 \mathrm{~nm}$ size were formed by facile hydrothermal method and later modified at BiOI NSs' atomically thin surface [120]. The AFM analysis showed that average thickness of BiOI was almost $0.9 \mathrm{~nm}$, proposing SL arrangement. 

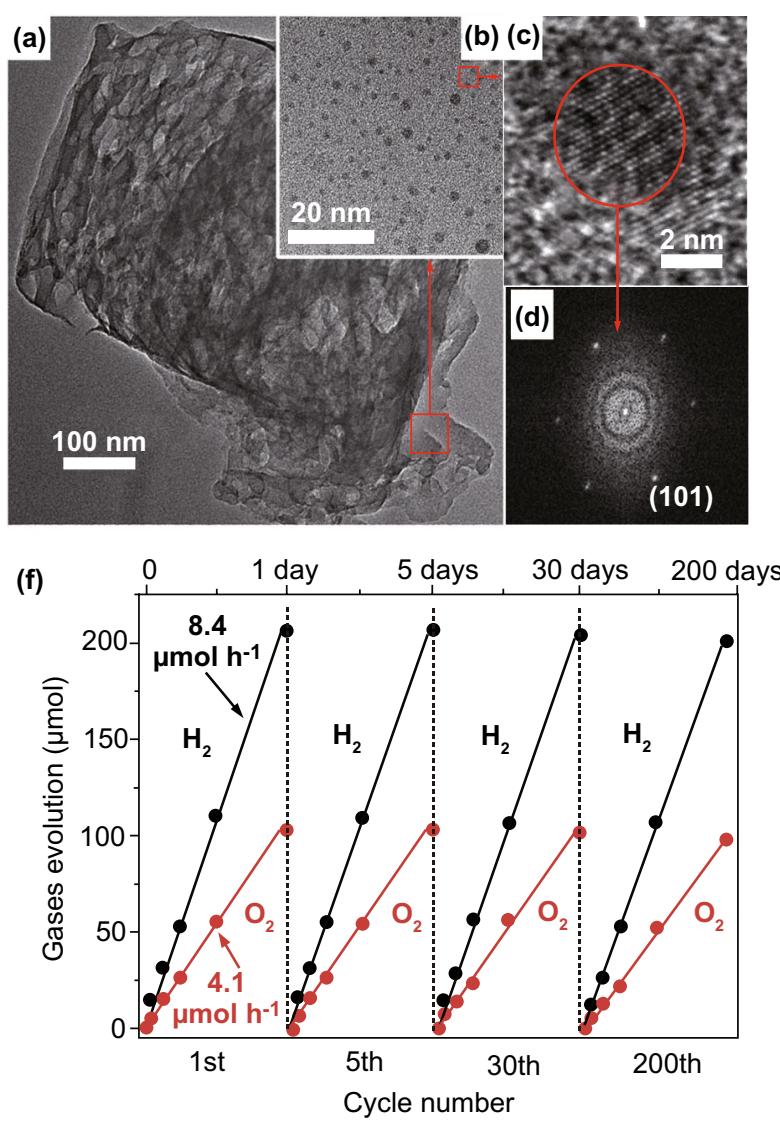
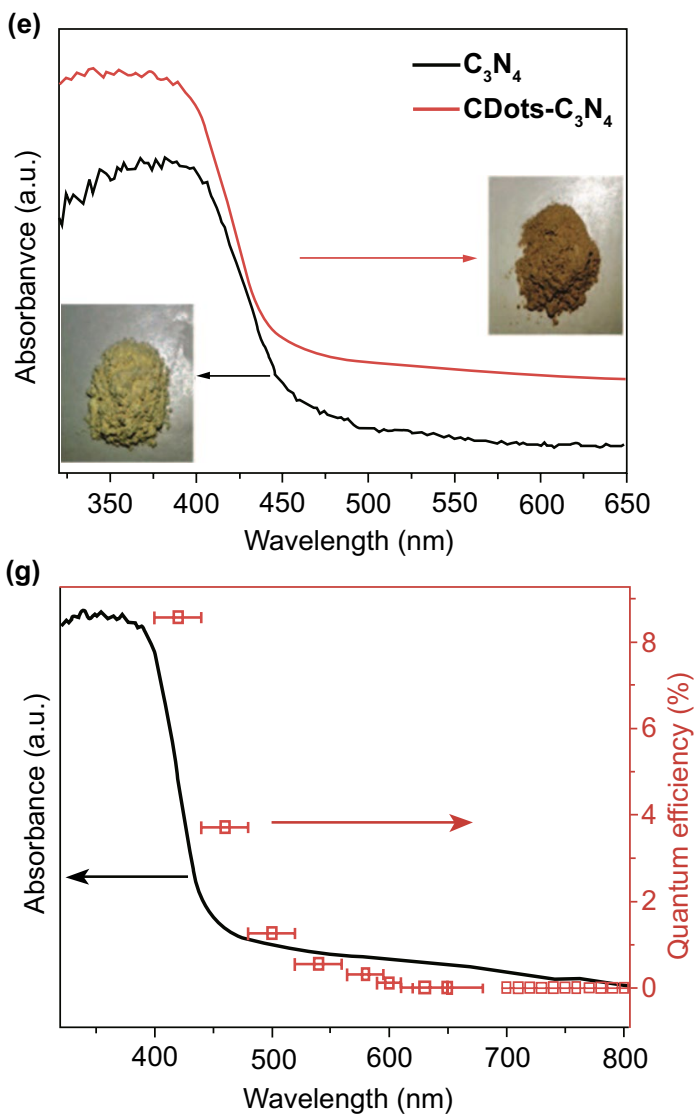

Fig. 15 a-d TEM of C-QDs/ $\mathrm{C}_{3} \mathrm{~N}_{4}$. e UV-Vis absorption spectrum of $\mathrm{C}_{3} \mathrm{~N}_{4}$ and C-QDs $/ \mathrm{C}_{3} \mathrm{~N}_{4}$ photo-catalysts. $\mathbf{f}$ Time period of $\mathrm{O}_{2}$ and $\mathrm{H}_{2}$ production from $\mathrm{H}_{2} \mathrm{O}$ after vis-light irradiation catalyzed via C-QDs $/ \mathrm{C}_{3} \mathrm{~N}_{4}$. $g$ Wavelength-based $\mathrm{QE}$ (red dots) of $\mathrm{H}_{2} \mathrm{O}$ splitting via C-QDs/ $\mathrm{C}_{3} \mathrm{~N}_{4}$. Adapted with permission from Ref. [215]

After N-CQDs, the N-CQDs/BiOI matters introduction displayed significantly extended lifetime of photo-generated charge carriers, as showed through time-resolved transient photo-luminescence (PL) decay and immediate photo-current. BiOI atomic-level configuration makes sure prominently quick bulk charge diffusion to surface and conjugated $\pi$-modified N-CQDs configuration, which effectively endorse surface charge separation, resulting in longer carrier duration. Therefore, the photo-catalytic activity and the N-CQDs/BiOI materials active species concentration enhanced considerably. Kang and co-authors also confirmed that C-QDs can work as chemical catalyst to really improve the photo-catalytic $\mathrm{H}_{2} \mathrm{O}$ splitting via $\mathrm{C}_{3} \mathrm{~N}_{4}$ (Fig. 15) [215]. Different from traditional single step $4 \mathrm{e}^{-}$reaction from $\mathrm{H}_{2} \mathrm{O}$ splitting, $2 \mathrm{e}^{-} / 2 \mathrm{e}^{-}$two-step path was followed by the C-QDs- $\mathrm{C}_{3} \mathrm{~N}_{4}$, where $\mathrm{C}_{3} \mathrm{~N}_{4}$ is added to transfer $\mathrm{H}_{2} \mathrm{O}$ to $\mathrm{H}_{2}$ and $\mathrm{H}_{2} \mathrm{O}_{2}$. Moreover, C-QDs are accountable in $\mathrm{H}_{2} \mathrm{O}_{2}$ decomposition and $\mathrm{O}_{2}$ evolution. Therefore, outstanding photo-catalytic $\mathrm{H}_{2} \mathrm{O}$ splitting effectiveness can be obtained, with $2.0 \%$ solar to $\mathrm{H}_{2}$ efficiency and robust stability in 200 recycle run after 200 days. Similarly, there are also some other systems concerning QD/2DMs hybridization to further enhance photo-catalytic efficiency, for example CdSe QDs, Zn-Ag-In-S QDs, NiS 2 QDs, and so on [216]. Such findings certainly verified superiority of QD modification, and QD/2D arrangement might be efficient substitute for configuration to get improved photo-catalytic performance.

\subsection{Single Atoms/2DMs Hybridization}

To further enhance photo-catalytic activity, size reduction of NPs to single atoms is possibly a probable plan. However, fraction of unsaturated coordination bonds of monoatom maximizes and enables a strong surface effect [217]. The first studied monoatom-based catalysis by Zhang et al. [218] 
became an interested topic in photo-catalysis direction. The monoatom-based photo-catalyst was based on isolated single atom on support materials surface, in the form of dispersion or coordination. Monoatoms-based strategy can improve photo-catalytic activity and offer another technique to alter the selectivity. As well, active single atom, chemical bonding in metal single atom, and 2DM NSs-based supports have become a strong and simpler charge transfer method. So, it is very attractive to create a single-atom/2DMs hybridization to obtain a high photo-catalytic activity [219]. Wu and co-workers [220] studied single Pt-atom as co-catalysts to advance photo-catalytic $\mathrm{H}_{2}$ evolution activity of $\mathrm{C}_{3} \mathrm{~N}_{4}$ NSs. A simple liquid phase reaction with $\mathrm{C}_{3} \mathrm{~N}_{4}$ and $\mathrm{H}_{2} \mathrm{PtCl}_{6}$-coupled low-temperature annealing was applied to form Pt-single atoms $/ \mathrm{C}_{3} \mathrm{~N}_{4}$. High-angle annular dark-field STEM (HAADF-STEM) was utilized to establish allocation and arrangement of Pt. The individual clear spots matching to Pt-atoms were viewed to be consistently dispersed on g- $\mathrm{C}_{3} \mathrm{~N}_{4}$, with $\sim 99.4 \% \mathrm{Pt}$ size of $>0.2 \mathrm{~nm}$, showing a Pt subsist completely as monoatoms. On the other hand, when the Pt loading quantity reaches $0.38 \%$, the Pt-atoms dispersion was denser and formed numerous sub-nanometer clusters. Extended X-ray absorption fine structure (EXAFS) spectroscopy was utilized to investigate local atomic configuration of the $\mathrm{Pt} / \mathrm{C}_{3} \mathrm{~N}_{4}$. The Pt-atoms coordination number was about 5 , through bond distance of $\sim 2.03 \AA$, exposing those Pt-atoms that were dispersed on the top of $\mathrm{C}_{3} \mathrm{~N}_{4}$ system. Following production of single Pt-atom/ $\mathrm{C}_{3} \mathrm{~N}_{4}$ arrangement, photo-catalytic $\mathrm{H}_{2}$-evolution activity was significantly enhanced. The $\mathrm{H}_{2}$-evolution rate of $\mathrm{Pt} / \mathrm{C}_{3} \mathrm{~N}_{4}(0.16 \mathrm{wt} \% \mathrm{Pt}$ loading) achieved was almost $318 \mu \mathrm{mol} \mathrm{h}{ }^{-1}$, about 50 times advanced, as compared to pristine- $\mathrm{C}_{3} \mathrm{~N}_{4}$. Simultaneously, single Pt-atom $/ \mathrm{C}_{3} \mathrm{~N}_{4}$ showed admirable stability for $\mathrm{H}_{2}$-evolution and isolated single Pt-atom still stays at $\mathrm{C}_{3} \mathrm{~N}_{4}$ after circulations. The desirable quality of UTAS, surface trap states of $\mathrm{C}_{3} \mathrm{~N}_{4}$ verified was basically changed because of isolated single Pt-atom that extends the carrier duration and provides more chances for $\mathrm{e}^{-} \mathrm{s}$ to engage in $\mathrm{H}^{+}$-reduction method. Moreover, separated single $\mathrm{Rh}$-atoms were spread on $2 \mathrm{D}-\mathrm{TiO}_{2} \mathrm{NSs}$ along with homogeneous $0.7 \mathrm{~nm}$ thickness via calcination, protonation, and coupled exfoliation method [221]. In HAADFSTEM image, separated brightest spots were observed that showed Rh-atoms but intermediate brightness spots symbolized Ti-atoms. The EXAFS showed that Rh species in single Rh-atoms $/ \mathrm{TiO}_{2}$ displayed an analogous chemical setting as $\mathrm{Rh}_{2} \mathrm{O}_{3}$ showed bonding to $\mathrm{O}$-atoms which was hence oxidized. Mono-Rh-atom co-catalysts were served as a reaction core for photo-catalytic-based $\mathrm{H}_{2}$-evolution, consistent with DFT simulations. So, $\mathrm{H}_{2}$-evolution rate was boosted 10 times as compared to pure $\mathrm{TiO}_{2} \mathrm{NSs}$. Although single atoms were engaged in catalysis, there were also existed numerous matters to be determined. Normally, the sustained content of monoatom was comparatively small and noble metal single-atom showed main types. It is very attractive to boost quantity of supported metal atoms with isolated single-atom arrangement and broaden it to other non-noble metals. Moreover, it is required to promote the stability, in order to meet potential industrial uses. The facts that single isolated metal atoms had high surface energy were shown, in which isolated metal atoms strongly cooperate with support surfaces. Through influenced metal atoms interactions with surface defects (elevated energy sites) on support, hybridization energy scheme might turn into a local minimum. Consequently, mono-metal atoms can be fastened as well as kept stable. Particularly in ultra-thin 2DMs, surface defects are liable to be generated because of ultra-large SSA and minute atomic flee energy. So, it is possible to construct monoatom anchored surface DR ultra-thin 2D arrangement to elevate photo-catalytic activity.

\subsection{Molecular/2DMs Hybridization}

Despite single isolated atoms, single molecule materials can also be used to engineer electronic structure by acting as a co-catalyst to enhance photo-catalytic activity [222]. Profiting from sub-nano-pores in $\mathrm{C}_{3} \mathrm{~N}_{4}$, molecular $\mathrm{TiO}_{2}$ was included into $\mathrm{C}_{3} \mathrm{~N}_{4}$ NSs by facile polycondensation of precursors with dicyandiamide and $\mathrm{TiO}_{2}$-ions [223] The morphology of clean ultra-thin TiO- $\mathrm{C}_{3} \mathrm{~N}_{4}$ NSs was obtained and confirmed by TEM analysis, with thickness of about 3-3.3 nm. From HAADF-STEM analysis and elemental mapping, $\mathrm{TiO}_{2}$ was originated to be consistently dispersed on $\mathrm{C}_{3} \mathrm{~N}_{4}$ framework with isolated format. These results suggested that molecular $\mathrm{TiO}_{2}$ was effectively built in $\mathrm{C}_{3} \mathrm{~N}_{4}$ framework equally. Local Ti-O geometrical and electronic structures in sub-nano-pores of $\mathrm{C}_{3} \mathrm{~N}_{4} \mathrm{NSs}$ were found through XAFS. Usually, anatase- $\mathrm{TiO}_{2}$ displayed welldefined triple pre-edge characteristics that can be recognized to distort $\mathrm{TiO}_{6}$ pattern with six coordinated $\mathrm{O}_{2}$-atoms. Dissimilar from $\mathrm{TiO}_{2}$ result, Ti-O in $\mathrm{C}_{3} \mathrm{~N}_{4}$ exhibited a single pre-edge feature with non-symmetric structure. The 
EXAFS showed that Ti-atoms were located in $\mathrm{C}_{3} \mathrm{~N}_{4} \mathrm{~h}^{+} \mathrm{s}$ that was coordinated with six $\mathrm{N}_{2}$-atoms in $\mathrm{C}_{3} \mathrm{~N}_{4}$ and one $\mathrm{O}_{2}$ atom out of plane in $\mathrm{C}_{3} \mathrm{~N}_{4}$. Benefiting from $\mathrm{TiO}_{2}$ molecule insertion, TiO- $\mathrm{C}_{3} \mathrm{~N}_{4}$ NSs displayed a narrowed $\mathrm{BG}$ in comparison with pristine $\mathrm{C}_{3} \mathrm{~N}_{4}$ with a reduction in $\mathrm{CB}$ position. It is resultant from more electron involvement of Ti-O into $\mathrm{C}_{3} \mathrm{~N}_{4}$ and enhances $\pi-\mathrm{e}^{-}$delocalization in conjugated structure. $\mathrm{TiO}-\mathrm{C}_{3} \mathrm{~N}_{4}$-engineered electronic structure can also enhance charge carrier separation. Therefore, TiO$\mathrm{C}_{3} \mathrm{~N}_{4}$ enhanced the photo-catalytic performance for $\cdot \mathrm{OH}$ generation and removal of pollutant. In photo-catalytic method, $\mathrm{h}^{+} \mathrm{s}$ transfer slower kinetics which brings massive carrier recombination in Achilles' heel of photo-catalytic conversion activity. Even though ultra-thin 2D-structure permits quick charge migration in bulk phase, lack of surface charge separation centers will also spoil overall photocatalytic activity. Utilizing strategy to encourage surface charge separation, particularly $\mathrm{h}^{+} \mathrm{s}$ transfer is extremely required. Instead, it looks feasible to utilize $\mathrm{H}_{2} \mathrm{O}$ soluble molecular materials as homogeneous co-catalyst and hence also optimize photo-catalytic performance. $\mathrm{Wu}$ and his research team [220] formed $\mathrm{H}_{2} \mathrm{O}$-soluble molecular trifluoroacetic acid (TFA) as co-catalysts to enhance photo-catalytic $\mathrm{H}_{2}$-evolution performance of $\mathrm{K}_{4} \mathrm{Nb}_{6} \mathrm{O}_{17} \mathrm{NSs}$. Taking advantage of reversible redox couple TFA./TFA- as well as high active intermolecular radical responses, TFA molecule served as strong $\mathrm{h}^{+} \mathrm{s}$-shuttle, allowing efficient move of the photo-generated $\mathrm{h}^{+} \mathrm{s}$ and ensuing elevated charge separation effectiveness. The TFA increment enhanced $\mathrm{H}_{2}$-evolution rate regularly and the maximum rate reached $6344 \mu \mathrm{mol} \mathrm{g}{ }^{-1} \mathrm{~h}^{-1}$, when TFA $/ \mathrm{K}_{4} \mathrm{Nb}_{6} \mathrm{O}_{17}$ molar ratio was 25.6. This optimum $\mathrm{H}_{2}$ yielding rate was $\sim 32$ times higher compared to pristine $\mathrm{K}_{4} \mathrm{Nb}_{6} \mathrm{O}_{17} \mathrm{NSs}$, certainly signifying this molecular co-catalyst. As solid-state co-catalysts, they are restricted from limited contact areas in co-catalysts and host photo-catalyst, and surface charge separation cannot be completely definite. When overmuch solid-state co-catalysts were anchored on photo-catalyst, the SASs will be covered that deficient in the satisfactory active sites easy to get reactant molecules. As a result, $\mathrm{H}_{2} \mathrm{O}$-soluble molecular materials such as molecular co-catalysts can equally disperse in the solution and give greatest available area to host photocatalysts. So, developed molecular co-catalyst approaches perhaps are a possible way in a competent separation of photo-generated carriers and therefore improve photo-catalytic performance.

\subsection{D-2D Stacking Materials Hybridization}

While constructing 2D-2D stacks, it is a largely applied method to boost photo-catalytic efficiency. Particularly for layered materials, lattice mismatch was reduced due to comparable layered structures, and 2D-2D stacking with close associates was formed. For example, Zhang and co-workers [139] studied SL $\mathrm{Bi}_{12} \mathrm{O}_{17} \mathrm{C}_{12}$ with surface $\mathrm{V}_{\mathrm{o}}$ via Li-intercalation-based exfoliation approach. Afterward, SLs $\mathrm{MoS}_{2}$ NSs were assembled onto SL- $\mathrm{Bi}_{12} \mathrm{O}_{17} \mathrm{C}_{12}$ through surface $\mathrm{V}_{\mathrm{v}}$ and build $\mathrm{Bi}-\mathrm{S}$ bonds in $\mathrm{Bi}_{12} \mathrm{O}_{17} \mathrm{Cl}_{2}$ and $\mathrm{MoS}_{2}$. TEM image well matched with an elemental mapping results proposed that numerous tiny $\mathrm{MoS}_{2}$ NSs were strongly anchored on a large $\mathrm{Bi}_{12} \mathrm{O}_{17} \mathrm{C}_{12}$ NS to make $2 \mathrm{D}$ stacking hetero-structure (Fig. 16); normal thickness of large size and small size NSs was $\sim 0.717$ and $0.686 \mathrm{~nm}$ that concurred with $\mathrm{Bi}_{12} \mathrm{O}_{17} \mathrm{Cl}_{2}$ and $\mathrm{MoS}_{2}$ SLs, in that order.

The atomic resolution HAADF-STEM analysis and resultant EELS elemental mapping showed that it was obviously studied and SLs- $\mathrm{MoS}_{2}$ were anchored selectively on $\left(\mathrm{Bi}_{12} \mathrm{O}_{17}\right)$ end faces to construct $\left(\mathrm{Cl}_{2}\right)-\left(\mathrm{Bi}_{12} \mathrm{O}_{17}\right)-\left(\mathrm{MoS}_{2}\right)$. As charge density surrounding $\left(\mathrm{Bi}_{12} \mathrm{O}_{17}\right)^{2+}$ layer was superior compared to $\left(\mathrm{Cl}_{2}\right)^{2-}$ layer, photo-generated $\mathrm{e}^{-} \mathrm{s}$ and $\mathrm{h}^{+} \mathrm{s}$ were ambitious to $\left(\mathrm{Bi}_{12} \mathrm{O}_{17}\right)^{2+}$ and $\left(\mathrm{Cl}_{2}\right)^{+}$end faces under irradiation, correspondingly. Photo-generated e-s flowed in between $\mathrm{MoS}_{2}$ SLs through formed Bi-S bonds and enable efficient charge separation (ultra-long duration of carrier $3446 \mathrm{~ns}$ ), as proofed through TA spectroscopy. Taking advantage of atomic size thickness, efficient directed interface charge separation, and plentiful $\mathrm{H}_{2}$-evolution sites in $\mathrm{MoS}_{2}$, acquired $\mathrm{MoS}_{2} / \mathrm{Bi}_{12} \mathrm{O}_{17} \mathrm{Cl}_{2}$ bilayers displayed great vis-light photo-catalytic $\mathrm{H}_{2}$-evolution performance. Using ascorbic acid as $\mathrm{h}^{+} \mathrm{s}$ sacrificial agent, $\mathrm{H}_{2}$-evolution rate can turn up $33 \mathrm{mmol} \mathrm{h}^{-1} \mathrm{~g}^{-1}$ and quantum effectiveness of $36 \%$ at $420 \mathrm{~nm}$. Other than $\mathrm{MoS}_{2} / \mathrm{Bi}_{12} \mathrm{O}_{17} \mathrm{Cl}_{2}$, there are several investigations about $2 \mathrm{D}$ stacking to optimize photo-catalytic efficiency, for instance $\mathrm{NiO} / \mathrm{Ca}_{2} \mathrm{Nb}_{3} \mathrm{O}_{10}$ [225], $\mathrm{MoS}_{2} /$ $\mathrm{TiO}_{2}$ [224], $\mathrm{MoS}_{2} / \mathrm{CdS}$ [226], $\mathrm{WS}_{2} / \mathrm{CdS}$ [226], $\mathrm{MoS}_{2} / \mathrm{C}_{3} \mathrm{~N}_{4}$ [227], $\mathrm{SnS}_{2} / \mathrm{C}_{3} \mathrm{~N}_{4}$ [228], $\mathrm{Fe}_{2} \mathrm{O}_{3} / \mathrm{C}_{3} \mathrm{~N}_{4}$ [229], $\mathrm{C}_{3} \mathrm{~N}_{4} / \mathrm{Bi}_{4} \mathrm{O}_{5} \mathrm{I}_{2}$, $\mathrm{ZnCr}-\mathrm{LDH} /$ layered titanate [230], and $\mathrm{ZnIn}_{2} \mathrm{~S}_{4} / \mathrm{MoSe}_{2}$. For example, a distinctive 2D stacking structure has more benefits. It increased the available area about planar interface in 2D/2D structures and reduced barriers for $\mathrm{e}^{-}$transportation via co-catalyst, and therefore promoted the interfacial charge transfer development through $\mathrm{e}^{-}$tunneling effect. Furthermore, these 2D thin layers can ease light blocking effect of 

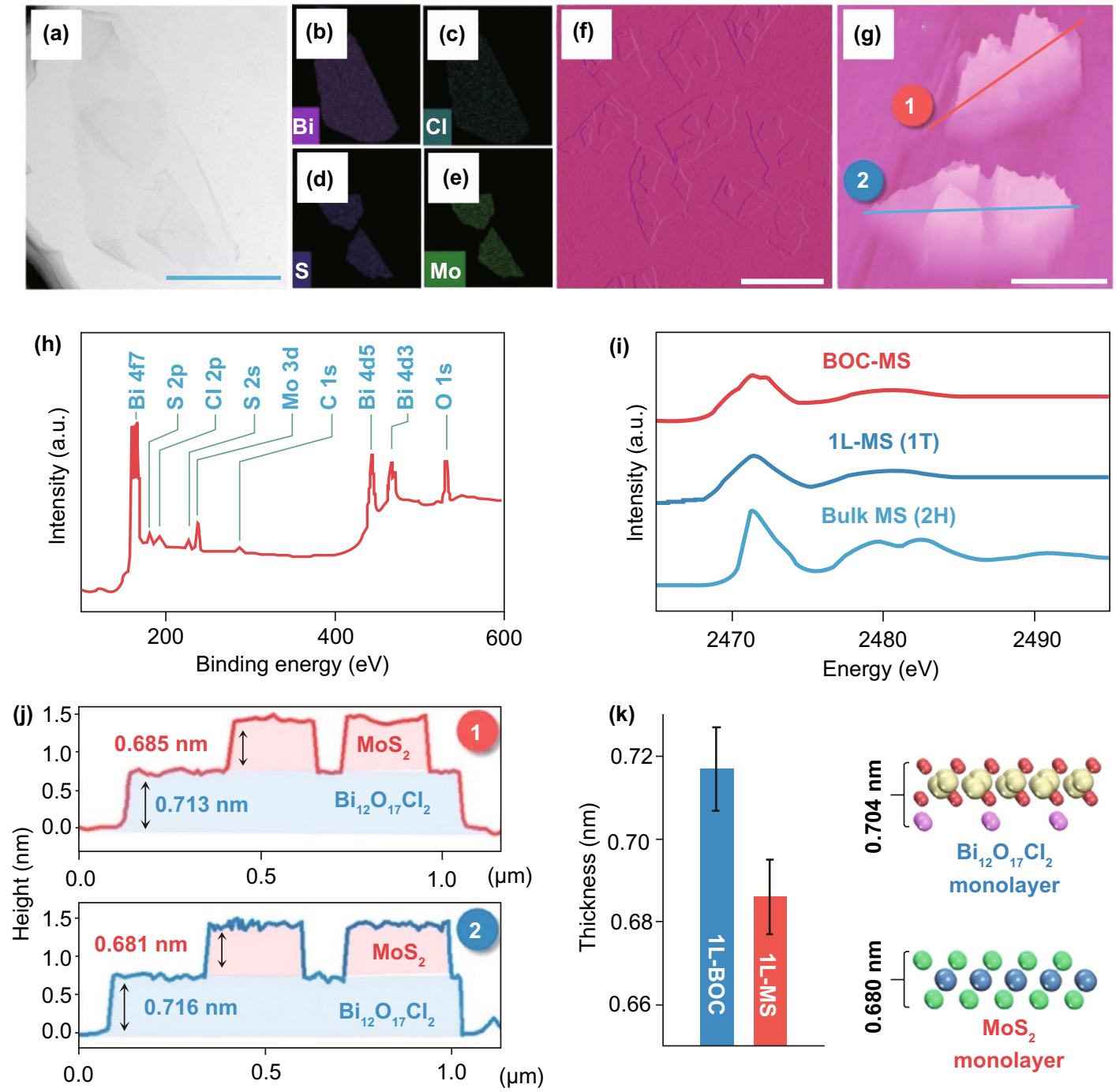

Fig. 16 a TEM top view analysis. b-e elemental mapping images, $\mathbf{f}$ XPS spectrum. h, i AFM analysis. $\mathbf{m}$ TEM images side view. $\mathbf{n}$ Atomic resolution HAADF-STEM image. o-s Respective EELS elemental maps of BOC-MS. g S K-edge XANES spectrum of BOC-MS, 1L-MS, and bulk MS. $\mathbf{j}$ Height profiles along lines in i. k Comparison of 1L-BOC and 1L-MS in BOC-MS average thicknesses. Error bars in (k) show s.d. over 100 self-sufficient AFM calculations. SLs $\mathrm{MoS}_{2}$ and $\mathrm{Bi}_{12} \mathrm{O}_{17} \mathrm{C}_{12}$ theoretical thicknesses. Adapted with permission from Ref. [224]

co-catalyst; therefore, sufficient light can contact the host photo-catalyst. It is required to alter $2 \mathrm{D}$-components and reinforce interfacial acting force to promote highly efficient 2D/2D photo-catalysts.

\section{Photo-catalytic Applications}

Based on the aforementioned consequences, ultra-thin 2DMs displayed huge benefits for the photo-catalysis from microstructure, BG, electronic configuration, and surface nature. Thus far, advanced ultra-thin 2DMs NSs were functional as photo-catalysts for diverse photo-catalytic uses. So, development of flexible photo-catalytic uses by $2 \mathrm{DMs}$ for $\mathrm{H}_{2} \mathrm{O}$ oxidation, $\mathrm{H}_{2}$-evolution, $\mathrm{CO}_{2}$ reduction, $\mathrm{N}_{2}$-fixation, organic synthesis, and pollutants removal will be explained in detail in the next section.

\subsection{Water $\left(\mathrm{H}_{2} \mathrm{O}\right)$ Oxidation}

Hydrogen $\left(\mathrm{H}_{2}\right)$ that has the highest energy density is measured as one of the potential energy carriers for storing solar energy in chemical bond energy form between two $\mathrm{H}$ atoms. 
In between different methods for conversion of sunlight and $\mathrm{H}_{2} \mathrm{O}$ into $\mathrm{H}_{2}$, photoelectrochemical (PEC) $\mathrm{H}_{2} \mathrm{O}$ splitting using SC photo-electrodes has gained most interest due to three main benefits:

1. Production of $\mathrm{H}_{2}$ and $\mathrm{O}_{2}$ at respective electrodes that eradicate separation problems.

2. Operation potential under environmental conditions.

3. Potential for manufacturing of a system which consists of just stable and copious inorganic materials.

Designing vis-light-active SCs for $\mathrm{H}_{2} \mathrm{O}$ splitting needs an appropriate BG and band position, efficient charge separation, fast charge movement, and longtime durability in aqueous solutions. An attractive design approach to fulfill
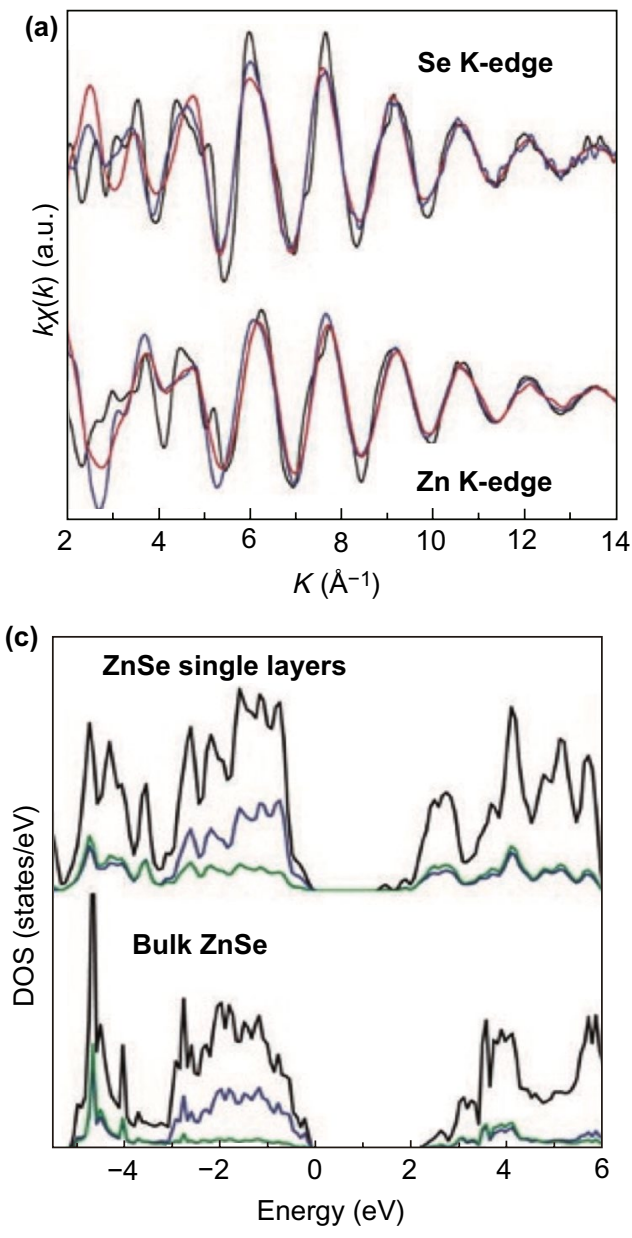

these requirements is to merge $2 \mathrm{D}$ materials (e.g., graphene, $\mathrm{MoS}_{2}, \mathrm{~g}-\mathrm{C}_{3} \mathrm{~N}_{4}$ ) with appropriate SCs. It is usually known that $\mathrm{H}_{2}$ is very potential green fuels with benefits, for example high specific energy, multiple use approaches, and pollution-free combustion product. It displayed magnificent view in prospect of sustainable energy use, if it is created through sustainable skill. The photo-catalytic $\mathrm{H}_{2} \mathrm{O}$ splitting into $\mathrm{H}_{2}$ and $\mathrm{O}_{2}$ is viewed as Holy Grail infield of chemistry through just sustainable solar light as energy input, photocatalysts as medium, and $\mathrm{H}_{2} \mathrm{O}$ as reaction source. As major forward step was accomplished, effectiveness of $\mathrm{H}_{2} \mathrm{O}$ splitting is still restricted in majority of photo-catalytic methods. Usually, $\mathrm{H}_{2} \mathrm{O}$-oxidation is efficiency, limited method in photo-catalytic $\mathrm{H}_{2} \mathrm{O}$ splitting schemes because of complex
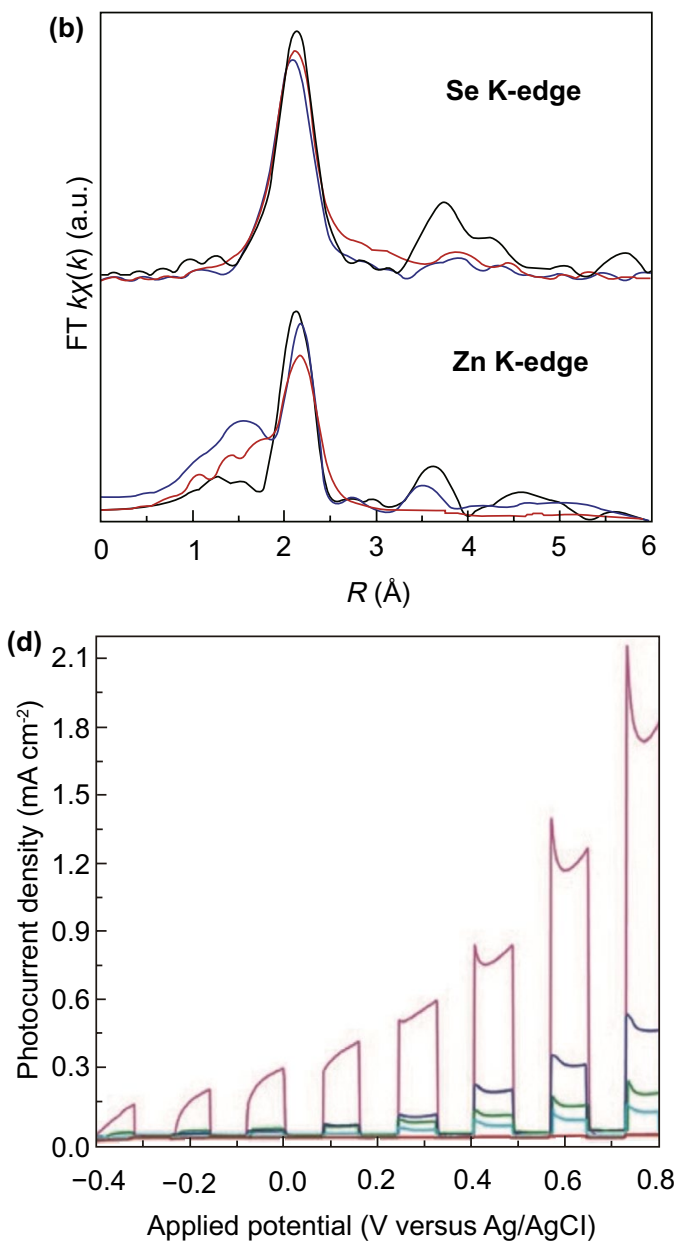

Fig. 17 a Se and Zn K-edge extended XAFS oscillation function k $\chi(\mathrm{k})$. b Equivalent Fourier transforms; red, blue, and black lines show ZnSe SLs, ZnSe-pa SLs, and bulk ZnSe, in that order. c Simulated DOS; black, blue, and olive lines show total, Se sp, and Zn sp state densities, correspondingly. d Photo-current density versus utilized potential curves in chopped $300 \mathrm{~W}$ Xe-lamp irradiation. Adapted with permission from Ref. [233] 
(a)

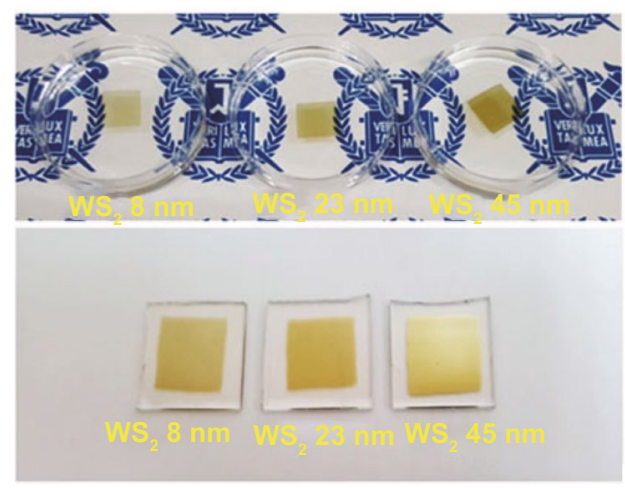

(c)

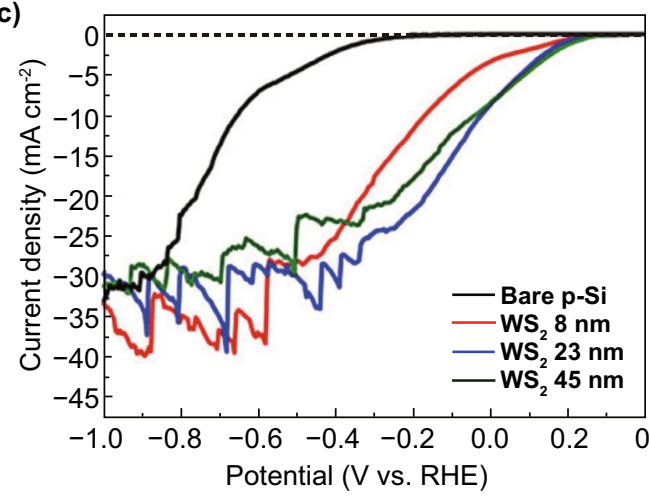

(b)

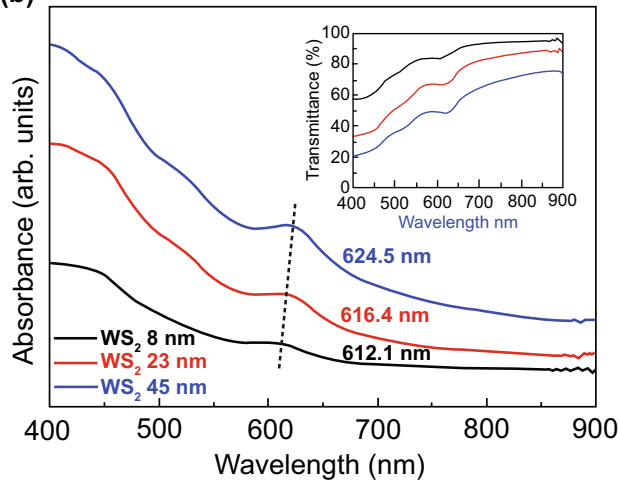

(d)

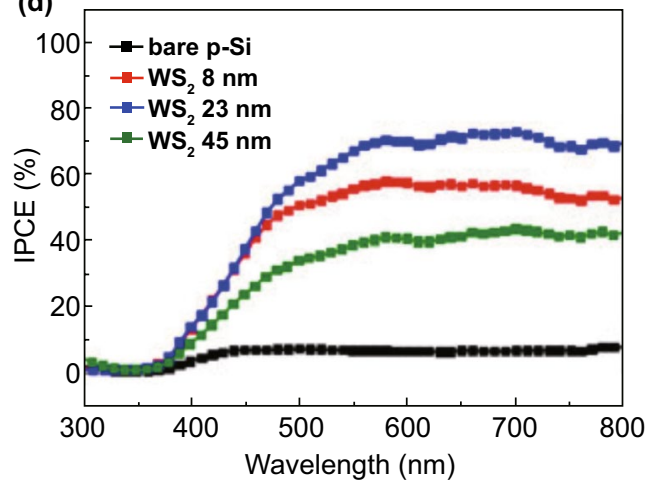

Fig. 18 a Photographs of $\mathrm{WS}_{2}$ films on glass with different thicknesses. b UV-Vis absorbance of $\mathrm{WS}_{2}$ different thin films. c PEC performance illustrated as J-V polarization curves for $\mathrm{WS}_{2}$ different films size. $\mathbf{d}$ IPCE measurements of $\mathrm{WS}_{2}$ different thin films size on p-Si photo-cathodes. Adapted with permission from Ref. [238]

four $\mathrm{h}^{+} \mathrm{s}$ complex redox method. So, it is highly in demand and imperative to propose photo-catalyst with robust solar $\mathrm{H}_{2} \mathrm{O}$-oxidation method. Recent studies illustrated that 2DMs-based photo-catalysts are very capable choice for solar $\mathrm{H}_{2} \mathrm{O}$-oxidation [231, 232]. The freestanding SLs ZnSe with four-atomic thickness was formed through ultrasonic exfoliation from lamellar hybrid intermediate $\left(\mathrm{Zn}_{2} \mathrm{Se}_{2}\right)(\mathrm{pa})$ (pa represents n-propylamine) [231]. The U-XAFS, local atomic structures, and electronic configurations of the $\mathrm{ZnSe}$ SLs were studied. For Zn K-edge $\mathrm{k} \chi_{(\mathrm{k})}$ oscillation curves as shown in (Fig. 17a), the ZnSe SLs displayed clear distinction comparative to ZnSe-pa SLs and bulk ZnSe, showing significant variation of local atomic arrangement. The R-space curves of $\mathrm{ZnSe}$ samples showed that peaks positioned at 2.11 and $3.63 \AA$ were attributed to the nearest $\mathrm{Zn}-\mathrm{Se}$ and next nearest $\mathrm{Zn}-\mathrm{Zn}$ coordination in bulk $\mathrm{ZnSe}$ (Fig. 17b). While $\mathrm{ZnSe}$ size was decreased to an atomic level, local atomic configuration experiences outstanding changes. ZnSe peak was shifted to $2.17 \AA$, and next the nearest $\mathrm{Zn}-\mathrm{Zn}$ distances (3.85 ^) were decreased. Simultaneously, Se-Se distances in SLs ZnSe NSs were extended from 4.012 to $4.11 \AA$ of bulk ZnSe. Such findings certainly showed reality of surface distortion in SL structure that reduced surface energy and allowed exceptional stability of ZnSe SLs. Moreover, surface deformation of SL ZnSe will consequently be in the form of enhanced DOSs at CB edge that might further make sure a high charge carrier transfer rate (Fig. 17c).

Taking advantages from SL structure with surface defects, ZnSe SLs show strong light absorption, enhanced charge separation effectiveness, and small charge transfer resistance. Consequently, SLs ZnSe NSs exhibited 195 times superior photo-catalytic performance compared to bulk $\mathrm{ZnSe}$ for $\mathrm{H}_{2} \mathrm{O}$ oxidation after Xe-lamp irradiation (Fig. 18d). As $\mathrm{H}_{2} \mathrm{O}$ oxidation to evolve $\mathrm{O}_{2}$ is completely $\mathrm{h}^{+}$-contributed reaction, enhancing $\mathrm{h}^{+}$-use rate perhaps is an efficient plan to enhance photo-catalytic $\mathrm{H}_{2} \mathrm{O}$-oxidation. Liu et al. [136] created several pore constructions in ultrathin $\mathrm{WO}_{3} \mathrm{NSs}$ through fast-heating approach on earlier exfoliated $\mathrm{WO}_{3} \cdot 2 \mathrm{H}_{2} \mathrm{O}$ NSs. As migration direction of the photo-generated $\mathrm{h}^{+} \mathrm{s}$ was along $\{001\}$ [2-4, 16-26] facets 
in the $x$-direction in $\mathrm{W}-\mathrm{O}-\mathrm{W}$ chains on $\mathrm{WO}_{3} \mathrm{NSs}$, with long itinerant pathway the photo-generated $\mathrm{h}^{+} \mathrm{s}$ certainly undergo several recombinations of charge carriers, which acutely prevent photo-catalytic performance. The created pores efficiently shorten diffusion way of $\mathrm{h}^{+} \mathrm{s}$ and conduce to $\mathrm{H}_{2} \mathrm{O}$ oxidation to make $\mathrm{O}_{2}$ at $\mathrm{WO}_{3}$ surface. Additionally, plentiful dangling bond along pore environment created good circumstances to make easy chemisorptions of molecular reaction that finally boosted $\mathrm{O}_{2}$-evolution kinetics. As a result, 18 times superior photo-catalytic $\mathrm{H}_{2} \mathrm{O}$ oxidation was attained for pore-rich $\mathrm{WO}_{3}$ ultra-thin NSs comparative to bulk $\mathrm{WO}_{3}$. It shows significant strategy to promote conversion efficiency by using ultra-thin $2 \mathrm{D}$ configuration to the photo-catalytic $\mathrm{H}_{2} \mathrm{O}$ oxidation. Additionally, many other photo-catalysts with ultra-thin 2D-structure can also show an exceptional photo-catalytic behavior toward $\mathrm{H}_{2} \mathrm{O}$ oxidation, for instance $\mathrm{SnS}_{2}$ [234], $\mathrm{SnSe}$ [235], $\mathrm{SnS}, \mathrm{Fe}_{2} \mathrm{O}_{3}$ [236], NiTi-LDH, and also engineered materials like $\operatorname{In}_{2} \mathrm{O}_{3}$ with $\mathrm{V}_{\mathrm{o}}$ engineered [84], Co-doped $\mathrm{In}_{2} \mathrm{~S}_{3}$ [145], pit-rich BiOCl [237], and so on.

\section{$6.2 \mathrm{H}_{2}$-Production}

As innovative investigation for photo-catalytic $\mathrm{H}_{2}$-evolution based on $\mathrm{TiO}_{2}$ in 1972, photo-catalytic $\mathrm{H}_{2}$-evolution from $\mathrm{H}_{2} \mathrm{O}$ was at front position of chemistry research to solve worldwide energy problem. Many amazing catalytic materials were used in photo-catalytic $\mathrm{H}_{2}$-production; majority are still said to have quite small photo-catalytic effectiveness, which could not meet the necessities of realistic uses on large industrial scale. Current research for the 2DMs established that rising 2D-SCs with suitable energy BG were a talented choice to get outstanding $\mathrm{H}_{2}$-evolution performance [239]. In case of abundant $\mathrm{SCs}$, the $\mathrm{Cu}_{2} \mathrm{O}$ with unique $\mathrm{CB}$, positioned at $\sim 0.7 \mathrm{~V}$ negative as compared to $\mathrm{H}_{2}$-evolution potential, is possibly a competent catalyst in $\mathrm{H}_{2}$-conversion from solar. To realize elevated $\mathrm{H}_{2}$-evolution efficiency, atomic-sized 2D-NSs, e.g., cubic $\mathrm{Cu}_{2} \mathrm{O}$, were formed. The AFM study showed thickness of about $0.62 \mathrm{~nm}$, related to four-atomic-level thickness of $\mathrm{Cu}_{2} \mathrm{O}$ in [01-1] direction. Therefore, surface energy of the cubic $\mathrm{Cu}_{2} \mathrm{O}$ was conformed to the order of $(111)<(100)<(110)$. As $(110)$ and $(01-1)$ surfaces are equivalent facets (01-1), facet in atomic-level thin $\mathrm{Cu}_{2} \mathrm{O}$ NSs also showed a great surface energy that brings a good activity. From considerably reduced thickness, atomically thin $\mathrm{Cu}_{2} \mathrm{O}$ NSs' electronic structure was distinct from bulk equivalent. Utilizing DFT simulations, an atomically thin $\mathrm{Cu}_{2} \mathrm{O}$ NSs showed really enhanced DOSs at edge of VB as compared to bulk $\mathrm{Cu}_{2} \mathrm{O}$. At similar time, extended $\mathrm{CB}$ edge was also studied in $2 \mathrm{D} \mathrm{Cu}_{2} \mathrm{O}$ NSs as compared to bulk $\mathrm{Cu}_{2} \mathrm{O}$, enlightening that atomic-level thick $\mathrm{Cu}_{2} \mathrm{O}$ has high carrier mobility and small BG. Profiting from such advantages, above 36 times higher photo-catalytic

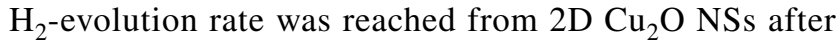
vis-light irradiation. These results certainly illustrated that 2DMs can bring huge advantage for $\mathrm{H}_{2}$-evolution as well as a series of extraordinary activities. In addition to the advancement in $\mathrm{H}_{2}$-evolution, more modification in ultrathin 2DMs structures was required. For instance, through doping $\mathrm{O}_{2}$ into $\mathrm{ZnIn}_{2} \mathrm{~S}_{4} \mathrm{NSs}, \mathrm{H}_{2}$-evolution rate of O-doped $\mathrm{ZnIn}_{2} \mathrm{~S}_{4}$ can attain $2120 \mu \mathrm{mol} \mathrm{h}{ }^{-1} \mathrm{~g}^{-1}$ from aqueous solution containing $0.25 \mathrm{~m} \mathrm{Na}_{2} \mathrm{SO}_{3}$ and $0.35 \mathrm{~m} \mathrm{Na}_{2} \mathrm{~S}$ after visible light illumination lacking any co-catalyst that was 4.5 times high as compared to pure $\mathrm{ZnIn}_{2} \mathrm{~S}_{4}$ [150].

Local atomic structures of formed materials were studied by XAFS. The $\mathrm{O}_{2}$ doping in $\mathrm{ZnIn}_{2} \mathrm{~S}_{4}$ NSs created high structure distortion through substitution of $\mathrm{O}_{2}$-atoms for sulfur. The engineered local atomic and electronic structure will experience observable deviation. The DFT simulation showed that $\mathrm{O}_{2}$ doping can boost DOS at VBM with respect to pure $\mathrm{ZnIn}_{2} \mathrm{~S}_{4}$, signifying creation of enhanced charge density about VBM. XPS results showed the valence spectra and estimated BG, where CBM and VBM of O-doped $\mathrm{ZnIn}_{2} \mathrm{~S}_{4}$ ultra-thin NSs show upshifting in comparison with pure $\mathrm{ZnIn}_{2} \mathrm{~S}_{4}$. So, average recovery duration of carriers for O-doped $\mathrm{ZnIn}_{2} \mathrm{~S}_{4} \mathrm{NSs}$ was about 1.53 factors long-lasting relative to pure $\mathrm{ZnIn}_{2} \mathrm{~S}_{4}$, attaining 110 ps. Hence, these advantages showed that $\mathrm{O}$-doped $\mathrm{ZnIn}_{2} \mathrm{~S}_{4}$ can show really enhanced photo-catalytic activity for $\mathrm{H}_{2}$-evolution. These findings showed that doping was an efficient policy to alter local electronic and atomic structure of 2DMs that can influence charge separation or migration and at the end optimizes $\mathrm{H}_{2}$-evolution.

Moreover, though considering major ultra-thin 2D-SCs NS materials lacks satisfactory $\mathrm{H}_{2}$-evolving sites, it is necessary to establish plentiful $\mathrm{H}_{2}$-evolution positions, for example, isolated Pt-atom or FL-TMDCs to more improve $\mathrm{H}_{2}$-evolution performance. Therefore, Wang and co-authors [227] studied FL $\mathrm{MoS}_{2}$ to $\mathrm{C}_{3} \mathrm{~N}_{4}$ NSs to improve photo-catalytic $\mathrm{H}_{2}$-evolution. In the formation method, $\mathrm{C}_{3} \mathrm{~N}_{4}$ was 

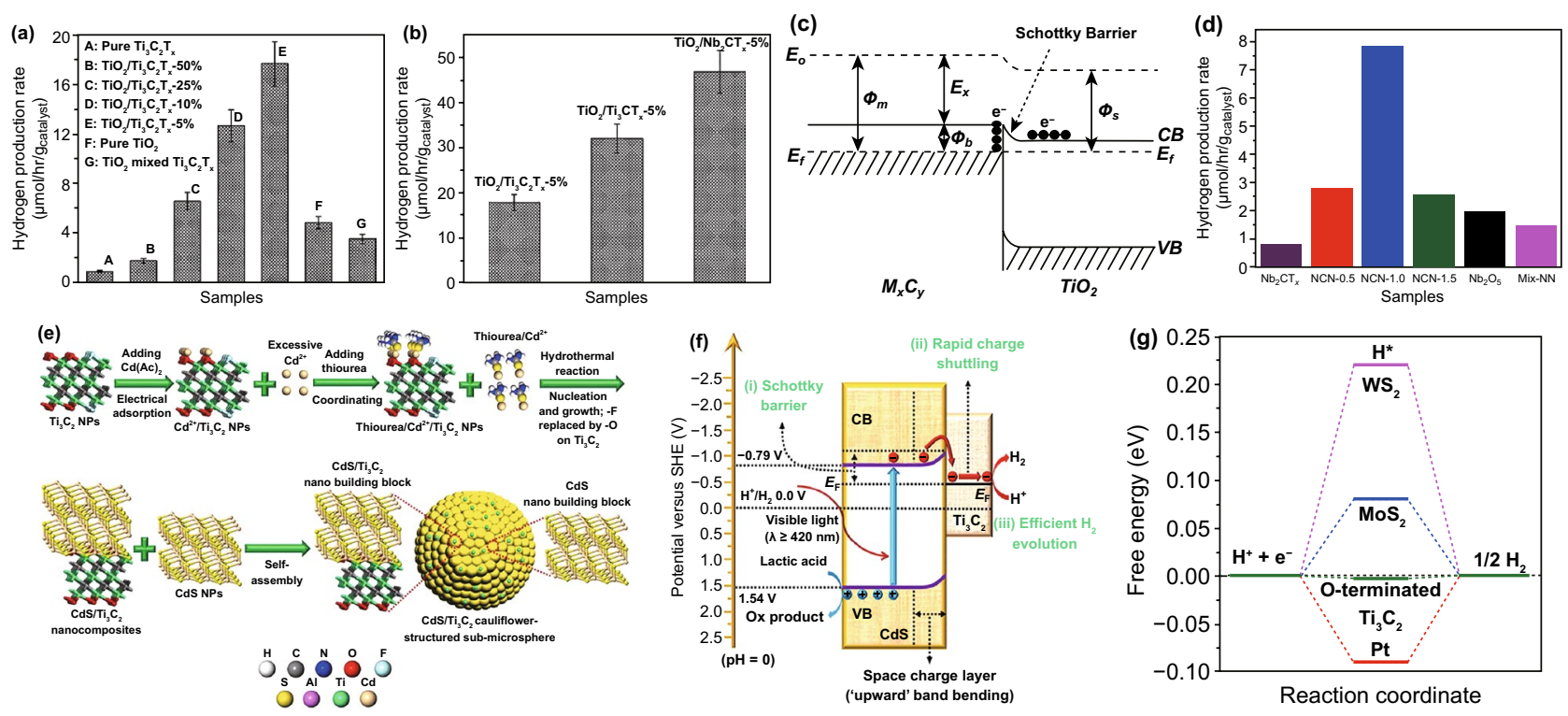

Fig. 19 a-c Photo-catalytic $\mathrm{H}_{2}$ formation rates of $\mathrm{TiO}_{2} / \mathrm{Ti}_{3} \mathrm{C}_{2} \mathrm{~T}_{x}$ and controlled samples. (a) Photo-catalytic HER activity of TiO $\mathrm{H}_{2}$ loaded with particular metal carbide MXene co-catalysts $\left(\mathrm{Ti}_{3} \mathrm{C}_{2} \mathrm{~T}_{x}, \mathrm{Ti}_{2} \mathrm{CT}_{x}\right.$, and $\left.\mathrm{Nb}_{2} \mathrm{CT}_{x}\right)$, (b) formation of SB at MXene/TiO 2 hetero-interface, and $(\mathbf{c}) \mathrm{Wang}$ et al. [240]. d Photo-catalytic HER of $\mathrm{Nb}_{2} \mathrm{O}_{5} / \mathrm{C} / \mathrm{Nb}_{2} \mathrm{C}$ samples (Tongming Su et al. [242]). e-g Fabrication method of CdS/Ti ${ }_{3} \mathrm{C}_{2}$ cauliflowerstructured sub-microsphere (e), charge separation and transfer in $\mathrm{CdS} / \mathrm{Ti}_{3} \mathrm{C}_{2}$ system after vis-light illumination (red and blue spheres illustrate photo-induced $\mathrm{e}^{-} \mathrm{s}$ and $\mathrm{h}^{+} \mathrm{s}$, correspondingly) (f) and at equilibrium potential HER free energy diagram (g). Adapted with permission from Ref. [242]

absorbed in $\left(\mathrm{NH}_{4}\right)_{2} \mathrm{MoS}_{4} \mathrm{H}_{2} \mathrm{O}$ solution and after that sulfidation was done through $\mathrm{H}_{2} \mathrm{~S}$ gas, at $350{ }^{\circ} \mathrm{C} . \mathrm{C}_{3} \mathrm{~N}_{4}$ and $\mathrm{MoS}_{2}$ have equivalent layer formation, which can reduce lattice mismatch and help planar development of $\mathrm{MoS}_{2}$ slabs after using $\mathrm{C}_{3} \mathrm{~N}_{4}$ surface. Consequently, an inorganic-organic 2D-2D stacking was formed using G-like thin-layer heterojunctions. Plentiful $\mathrm{H}_{2}$-evolution sites were formed via $\mathrm{FL}$ $\mathrm{MoS}_{2}$ NSs. Moreover, dispersed $\mathrm{MoS}_{2}$ thin layers on $\mathrm{C}_{3} \mathrm{~N}_{4}$ NSs surface can provide the superior effectiveness compared to multilayer $\mathrm{MoS}_{2}$ because of e-tunneling effect via $\mathrm{MoS}_{2}$ thin layers from reaction interfaces. On the basis of support charge separation and plentiful $\mathrm{H}_{2}$-evolving sites persuaded through FL MoS 2 NSs, acquired $\mathrm{MoS}_{2} / \mathrm{C}_{3} \mathrm{~N}_{4} 2 \mathrm{D}$ junctions exhibited better photo-catalytic $\mathrm{H}_{2}$-evolution activity in comparison with pristine $\mathrm{C}_{3} \mathrm{~N}_{4}$. The $0.2 \mathrm{wt} \% \mathrm{MoS}_{2}$ is the best sample that illustrated the highest $\mathrm{H}_{2}$-evolving rate, with an obvious $2.1 \%$ QY recorded at $420 \mathrm{~nm}$. Moreover, $\mathrm{WS}_{2}$ can be utilized as a catalyst in $\mathrm{WS}_{2} / \mathrm{p}$-type $\mathrm{Si}$ photo-cathode hetero-junctions. Kwon et al. [238] examined $\mathrm{WS}_{2} / \mathrm{p}$-Si photo-cathode for photo-catalytic based HER. Figure 18a shows the as-prepared $\mathrm{WS}_{2}$ thin film color changes from yellow to brown with increasing thickness, whereas absorbance of film regularly enhances. However, absorption peak position almost remains constant (Fig. 18b). The PEC demonstrated that photo-catalytic HER performance for $23 \mathrm{~nm} \mathrm{WS} / 2 / \mathrm{p}-\mathrm{Si}$ showed the maximum current density of $8.375 \mathrm{~mA} \mathrm{~cm}^{-2}$ at $0 \mathrm{~V}$ and $72 \%$ incident photon to current conversion efficiency (IPCE) (Fig. 18c, d). So, it shows that merger of TMDs ( $\mathrm{WS}_{2}, \mathrm{MoS}_{2}$ ), along traditional SCs, for

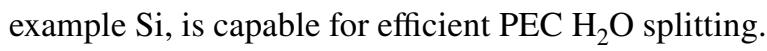

Similarly, Wang et al. [240] studied physicochemical nature of $\mathrm{Ti}_{3} \mathrm{C}_{2} \mathrm{~T}_{x}$ MXene coupled with $\mathrm{TiO}_{2}$ for photo-catalytic HER (Fig. 19a) [241]. The $5 \mathrm{wt} \%$ content of $\mathrm{Ti}_{3} \mathrm{C}_{2} \mathrm{~T}_{x}$ in $\mathrm{TiO}_{2} / \mathrm{Ti}_{3} \mathrm{C}_{2} \mathrm{~T}_{x}$ nano-composite showed a $400 \%$ augmentation for the photo-catalytic HER than rutile $\mathrm{TiO}_{2}$. Therefore, $\mathrm{Ti}_{3} \mathrm{C}_{2} \mathrm{~T}_{x}$ gives a $2 \mathrm{D}$-podium to interrelate with consistently fabricated $\mathrm{TiO}_{2}$ to make possible partition of photo-generated $\left(\mathrm{e}^{-}-\mathrm{h}^{+}\right)$-pairs to slow charge recombination. Fascinatingly, $\mathrm{TiO}_{2}$ NPs were well dispersed at $\mathrm{Ti}_{3} \mathrm{C}_{2} \mathrm{~T}_{x}$ surface without harsh aggregation. Additionally, $\mathrm{Ti}_{3} \mathrm{C}_{2} \mathrm{~T}_{x}, \mathrm{Nb}_{2} \mathrm{CT}_{x}$, and $\mathrm{Ti}_{2} \mathrm{CT}_{x}$ were also utilized as reducing co-catalysts after reacting with $\mathrm{TiO}_{2}$ and increase photo-activity in the HER [240]. Fascinatingly, $\mathrm{Nb}_{2} \mathrm{CT}_{x}$ and $\mathrm{Ti}_{2} \mathrm{CT}_{x}$ show better HER catalytic activity as compared to $\mathrm{Ti}_{3} \mathrm{C}_{2} \mathrm{~T}_{x}$, while coupling with $\mathrm{TiO}_{2}$ as co-catalysts (Fig. 19b). It can be dragged through Schottky barrier (SB) and work function of every 
MXene, where $\mathrm{Nb}_{2} \mathrm{CT}_{x}$ shows the maximum work function $(\sim 4.1 \mathrm{eV})$ for direct proof of the highest HER activity. In reaction system, $\mathrm{TiO}_{2}$ surface was excited after irradiation with light to generate $\mathrm{e}^{-} \mathrm{s}$ and $\mathrm{h}^{+} \mathrm{s}$. As a result of different Fermi levels of MXene and $\mathrm{TiO}_{2}$, photo-generated $\mathrm{e}^{+} \mathrm{s}$ was transferred from $\mathrm{CB}$ of $\mathrm{TiO}_{2}$ to MXene. Furthermore, adding positive charges in $\mathrm{TiO}_{2}$ and negative charges in MXene, $\mathrm{CB}$ and VB were turned upward (Fig. 19c), leading to form SB at $\mathrm{TiO}_{2} / \mathrm{MXene}$ hetero-interfaces to avoid $\mathrm{e}^{-} \mathrm{s}$ from relocating back to $\mathrm{TiO}_{2}$. In addition to $\mathrm{SB}$ and fast $\mathrm{e}^{-}$shuttling, build-up of $\mathrm{e}^{-} \mathrm{s}$ on MXene will respond to $\mathrm{H}^{+}$-ions to create $\mathrm{H}_{2}$.

In addition, Su et al. [242] formed $\mathrm{Nb}_{2} \mathrm{O}_{5} / \mathrm{C} / \mathrm{Nb}_{2} \mathrm{C}$ (MXene) hybrid nano-composites (NCN- $x$ ) through oxidizing $\mathrm{Nb}_{2} \mathrm{CT}_{x}$ surface at various durations $(x=0.5,1$, and $1.5 \mathrm{~h}$ ) to produce a $\mathrm{Nb}_{2} \mathrm{O}_{5}$ layer via utilizing $\mathrm{CO}_{2}$ as gentle oxidant. Optimum duration of oxidation of $1 \mathrm{~h}$ photo-activity of NCN-1.0 for HER was four times higher as compared to pure $\mathrm{Nb}_{2} \mathrm{O}_{5}$ (Fig. 19d), which was created from close interfacial junction in conducting $\mathrm{Nb}_{2} \mathrm{C}$ co-catalysts and $\mathrm{SC} \mathrm{Nb}_{2} \mathrm{O}_{5}$ for exceptional $\left(\mathrm{e}^{-}-\mathrm{h}^{+}\right)$-separation. This showed generation of transition metal carbides as feasible co-catalysts in solar to chemical energy conversion. Other than MO photo-catalysts, hybridization of TMDCs with MXene has also turned into an effervescent area of catalysis research in energy conversion. Qiao and co-workers also studied metal sulfide $/ \mathrm{Ti}_{3} \mathrm{C}_{2}$ (metal sulfide: $\mathrm{CdS}, \mathrm{ZnS}$, and $\mathrm{Zn}_{x} \mathrm{Cd}_{1-x} \mathrm{~S}$ ) nano-hybrid photo-catalysts (Fig. 19e) [243]. The CdS/ $\mathrm{Ti}_{3} \mathrm{C}_{2}$ composite records high vis-light HER photo-activity $\left(14,342 \mathrm{~mol} \mathrm{~h}^{-1} \mathrm{~g}^{-1}, 136.6\right.$ times that of bare $\left.\mathrm{CdS}\right)$ with surprisingly large apparent QY value $40.1 \%$ at $420 \mathrm{~nm}$, showing that it was one of the most excellent noble metal-free metal sulfide photo-catalytic system. The DFT simulations showed that $\mathrm{O}_{2}$-terminated $\mathrm{Ti}_{3} \mathrm{C}_{2}$ was talented co-catalyst derived from its marvelous HER activity, outstanding metallic conductance, and enviable Fermi levels. Basically, such investigation formed a novel view for scheming high-efficient and cost-efficient solar $\mathrm{H}_{2} \mathrm{O}$ splitting utilizing metal chalcogenide photo-catalysts and photo-electrodes. Moreover, MXene-based composite with metal-free $\mathrm{g}-\mathrm{C}_{3} \mathrm{~N}_{4}$ photo-catalysts has a new start of attention in kingdom of renewable energy formation. Shao et al. [244] formed a $\mathrm{Ti}_{2} \mathrm{C} / \mathrm{g}-\mathrm{C}_{3} \mathrm{~N}_{4}$ photo-catalyst by use of thermal annealing of melamine with the $2 \mathrm{D}-\mathrm{Ti}_{2} \mathrm{C}$ NSs for the outstanding HER activity. Optimum $0.4 \mathrm{wt} \%$ of $\mathrm{Ti}_{2} \mathrm{C}$ provides an elevated $\mathrm{H}_{2}$-production rate of $950 \mathrm{~mol} \mathrm{~h}^{-1} \mathrm{~g}^{-1}$ with apparent QY of $4.3 \%$ at $420 \mathrm{~nm}$.
Enhanced photo-catalytic efficiency was observed by efficient charge transfer and separation due to the existence of $\mathrm{SB}$ to decrease $\mathrm{H}^{+}$to $\mathrm{H}_{2}$. Similarly, Sun et al. [75] also studied g- $\mathrm{C}_{3} \mathrm{~N}_{4} / \mathrm{Ti}_{3} \mathrm{C}_{2} \mathrm{~T}_{x}$ photo-catalysts, in which $\mathrm{Ti}_{3} \mathrm{C}_{2} \mathrm{~T}_{x}$ with $\mathrm{O}$-surface terminations enhances the separation of charges for improvement of $105 \%$ in the HER activity with apparent QY of $1.27 \%$. The DFT simulations support it and showed that O-terminated $\mathrm{Ti}_{3} \mathrm{C}_{2}$ with $25 \% \mathrm{H}$-atoms presents obvious free energy as low as $0.011 \mathrm{eV}$ [241]. Giorgio Carraro et al. [81] explored enhanced $\mathrm{H}_{2}$ evolution via photo-reforming of sustainable oxygenates using nano-structure $\mathrm{Fe}_{2} \mathrm{O}_{3}$ polymorphs. They studied that Fe(III) oxide polymorphs, $\beta$ - and $\varepsilon-\mathrm{Fe}_{2} \mathrm{O}_{3}$, have notable performance in solar spectrum for $\mathrm{H}_{2}$ production from solutions of renewable oxygenates (i.e., ethanol, glycerol, glucose). For $\beta-\mathrm{Fe}_{2} \mathrm{O}_{3}$ and $\varepsilon-\mathrm{Fe}_{2} \mathrm{O}_{3}, \mathrm{H}_{2}$ evolution rates up to 225 and $125 \mathrm{mmol} \mathrm{h}^{-1} \mathrm{~m}^{-2}$ are gained, along significant better activities in regard to commonly study $\alpha-\mathrm{Fe}_{2} \mathrm{O}_{3}$.

\subsection{Reduction of $\mathrm{CO}_{2}$}

Other than photo-catalytic $\mathrm{H}_{2}$-evolution, $\mathrm{CO}_{2}$ reductions to produce hydrocarbon fuels over photo-catalysts were observed as an efficient way to concurrently reduce energy crisis and greenhouse cause. Basically, combustion of fossil fuel develops a great quantity of $\mathrm{CO}_{2}$, causes an increase in greenhouse effect due to unstoppable increase in $\mathrm{CO}_{2}$. Significantly, conversion of $\mathrm{CO}_{2}$ to valuable fuels is an alarming challenge in the recent time. Certainly, $\mathrm{CO}_{2}$ composed a fundamental $\mathrm{C} 1$ building element for chemical industries, but its thermodynamic stability and very high kinetic blocked its broad industrial uses. The undeniable solution for this mystery is to discover C-neutral fuels for low-C market to a sustainable future without environment disadvantages [245]. Usually, the $\mathrm{CO}_{2}$ molecules are very stable with $\mathrm{C}=\mathrm{O}$ bond dissociation energy higher than $\sim 750 \mathrm{~kJ} \mathrm{~mol}^{-1}$. Thus, in $\mathrm{CO}_{2}$ photo-reduction method, higher energy is required to split $\mathrm{O}=\mathrm{C}=\mathrm{O}$ structure, that is very demanding compared to $\mathrm{H}_{2} \mathrm{O}$ splitting into $\mathrm{H}_{2}$. Gateway for creation of a $\mathrm{CO}^{2--}$ intermediate through single $\mathrm{e}^{-}$transmission to activate a $\mathrm{CO}_{2}$ was observed as a ratelimited step to ensue proton-concerned reduction method. A theoretical potential of $-1.9 \mathrm{~V}$ versus NHE is needed for initial startup, and a superior over-potential is necessary for actual utilized potentials. Furthermore, multiple 
proton-coupled $\mathrm{e}^{-}$transfer methods are concerned in $\mathrm{CO}_{2}$ activation and reaction ways were quite difficult and varied with the synthesis of different products. In accordance with diverse number of injected $\mathrm{e}^{-} \mathrm{s}\left(2 \mathrm{e}^{-}, 4 \mathrm{e}^{-}, 6 \mathrm{e}^{-}\right.$, and $8 \mathrm{e}^{-}$), the products, for example, are $\mathrm{CO}, \mathrm{HCHO}, \mathrm{CH}_{3} \mathrm{OH}$, and $\mathrm{CH}_{4}$, correspondingly. Moreover, competition exists for $\mathrm{CO}_{2}$ photo-reduction and $\mathrm{H}_{2} \mathrm{O}$ reduction, because $\mathrm{H}_{2} \mathrm{O}$ reduction to produce $\mathrm{H}_{2}$ is energetically more encouraging that confines products selectivity. Current research explained that ultra-thin 2DMs-based photo-catalysts also displayed outstanding $\mathrm{CO}_{2}$ photo-reduction. For instance, $\mathrm{Bi}_{2} \mathrm{WO}_{6}$ layers with single-unit-cell thickness were formed through a lamellar hybrid intermediate plan [246]. Sodium oleate was used to provide oleate ions, thus to relate to $\mathrm{Bi}^{3+}$ via electrostatic interaction. Afterward, lamellar Bioleate complexes were formed through self-assembly with tail-to-tail or head-to-head bilayer array of oleate ions to construct a meso-structure. While $\mathrm{Na}_{2} \mathrm{WO}_{4}$ was inserted and treated with hydrothermal method, $\mathrm{Bi}_{2} \mathrm{WO}_{6}$ was formed and lamellar meso-structure was self-exfoliated into a single-unit-cell layer. As-synthesized single-unit-cell $\mathrm{Bi}_{2} \mathrm{WO}_{6}$ layer was used in photo-catalyst for $\mathrm{CO}_{2}$ photoreduction and a $300 \mathrm{~W}$ Xe lamp. The $\mathrm{Bi}_{2} \mathrm{WO}_{6}$ powder was suspended in $\mathrm{H}_{2} \mathrm{O}$ along highly pure $\mathrm{CO}_{2}$ gas constantly bubbled during solution for measurements. An average rate of $75 \mu \mathrm{mol} \mathrm{g} \mathrm{g}^{-1} \mathrm{~h}^{-1}$ was obtained in methanol synthesis over single-unit-cell $\mathrm{Bi}_{2} \mathrm{WO}_{6}$ layers for 5-h analysis that was almost 3 and 125 times higher comparative to $\mathrm{Bi}_{2} \mathrm{WO}_{6}$ nano-crystals and bulk $\mathrm{Bi}_{2} \mathrm{WO}_{6}$ correspondingly. Following six cycles, photo-reduction effectiveness remains the same and is not effected by any deterioration and shows an outstanding photo-stability. Recent research initiates that an excellent photo-catalytic performance was resulting from the novel geometrical configuration of single-unitcell $\mathrm{Bi}_{2} \mathrm{WO}_{6}$ layers, as follows:

1. Initially ultra-large SSAs of single-unit-cell $\mathrm{Bi}_{2} \mathrm{WO}_{6}$ layers make sure 3 factors higher capacitance in $\mathrm{CO}_{2}$ adsorption that was a significant tip for $\mathrm{CO}_{2}$ activation and reduction.

2. The single-unit-cell thicknesses give advance charge separation and extend carrier duration as testified through time-resolved fluorescence emission spectrum calculations.

3. Single-unit-cell thickness carries almost higher DOS at $\mathrm{CB}$ edge as well as boosted surface charge density and hence promoted the $2 \mathrm{D}$-conductivity.
Research certainly showed benefits of 2DMs photo-catalyst for $\mathrm{CO}_{2}$ photo-reduction. Adsorption of $\mathrm{CO}_{2}$ is a significant condition for $\mathrm{CO}_{2}$ photo-reduction, which considerably influences $\mathrm{e}^{-}$transfer method. Here, it is attractive to find suitable approach to boost adsorption site for $\mathrm{CO}_{2}$ adsorption and generate strong contact to make possible $\mathrm{e}^{-} \mathrm{s}$ transfer for efficient activation. Research concluded that generating surface defect sites is possibly another way [97]. Although with controlled synthesis, ZnAl-LDH ultra-thin NSs with thicknesses of 2.7 and $4.1 \mathrm{~nm}$ and bulk ZnAl-LDH with almost $210 \mathrm{~nm}$ thicknesses can be synthesized that are known as $\mathrm{ZnAl}^{-1}, \mathrm{ZnAl}^{-2}$, and $\mathrm{ZnAl}^{-3}$, correspondingly. While bulk $\mathrm{ZnAl}-\mathrm{LDH}$ thickness is reduced to ultra-thin configuration, density of $\mathrm{V}_{\mathrm{o}}$ defects is increased, so reduces coordination number of nearby $\mathrm{Zn}$-ion and initiates several coordinative unsaturated $\mathrm{Zn}$-ions. So, $\mathrm{Zn}^{+}-\mathrm{V}_{\mathrm{o}}$-complexes are built in ZnAl-LDH NSs as supported through XAFS, ESR, and PAS spectra calculations. In $\mathrm{CO}_{2}$ photo-reduction route, synthesized $\mathrm{Zn}^{+}-\mathrm{V}_{\mathrm{o}}$ complexes can function as entrapping positions to encourage $\mathrm{CO}_{2}$ adsorption. Simultaneously, EIS and DFT simulation showed that $\mathrm{Zn}^{+}-\mathrm{V}_{\mathrm{o}}$ complexes can provide $\mathrm{e}^{-} \mathrm{s}$ entrapping sites to improve charge separation effectiveness and make easy $\mathrm{e}^{-}$-transfer to $\mathrm{CO}_{2}$. Therefore, defectrich ZnAl-LDH NSs showed great photo-catalytic activity for conversion of $\mathrm{CO}_{2}$ into $\mathrm{CO}$ through a $2 \mathrm{e}^{-}$method, with $7.6 \mu \mathrm{mol} \mathrm{g}^{-1} \mathrm{~h}^{-1}$ conversion efficiency for $\mathrm{ZnAl}-1$. Further improved $\mathrm{CO}_{2}$ conversion competency, selectivity, and developed stability obtained by Xie et al. [154] tuned $\mathrm{V}_{\mathrm{v}}$ into a single-unit-cell o- $-\mathrm{BiVO}_{4}$ and then it is used as a photo-catalyst for $\mathrm{CO}_{2}$-reduction. Using cetyltrimethylammonium bromide, a lamellar hybrid half way plan was used for the formation of o- $\mathrm{BiVO}_{4}$ layers with single-unit-cell thickness. Regulating reaction time and temperature, $\mathrm{V}_{\mathrm{v}}$-rich and $\mathrm{V}_{\mathrm{v}}$-poor o- $\mathrm{BiVO}_{4}$ atomic layers were formed with [001] direction. Atomically thick o- $\mathrm{BiVO}_{4} \mathrm{NSs}$ showed AFM and TEM results. As proved through PAS and XRF, $\mathrm{V}_{\mathrm{v}}$ with discrete levels was formed on $\mathrm{V}_{\mathrm{v}}$-poor o- $\mathrm{BiVO}_{4}$ and $\mathrm{VV}$ rich o- $\mathrm{BiVO}_{4} \mathrm{NSs}$ surface. $\mathrm{V}_{\mathrm{v}}$ showed a significant function in photo-catalytic method for $\mathrm{CO}_{2}$-reduction. Firstly, $\mathrm{V}_{\mathrm{v}}$ created a new defect concentration in BG and enhanced $\mathrm{h}^{+} \mathrm{s}$ level near the Fermi level. Therefore, light harvesting of $\mathrm{V}_{\mathrm{v}}$-rich o- $\mathrm{BiVO}_{4}$ was improved and electronic conductance was better. The $\mathrm{V}_{\mathrm{v}}$-rich $\mathrm{o}-\mathrm{BiVO}_{4}$ showed an increased $\mathrm{CO}_{2}$-adsorption capacitance and stronger surface hydrophilicity comparative to $\mathrm{V}_{\mathrm{v}}$-poor $\mathrm{o}-\mathrm{BiVO}_{4}$; this was certainly useful to $\mathrm{CO}_{2}$ reduction process. Finally, $\mathrm{V}_{\mathrm{v}}$ improved charge 
(a)

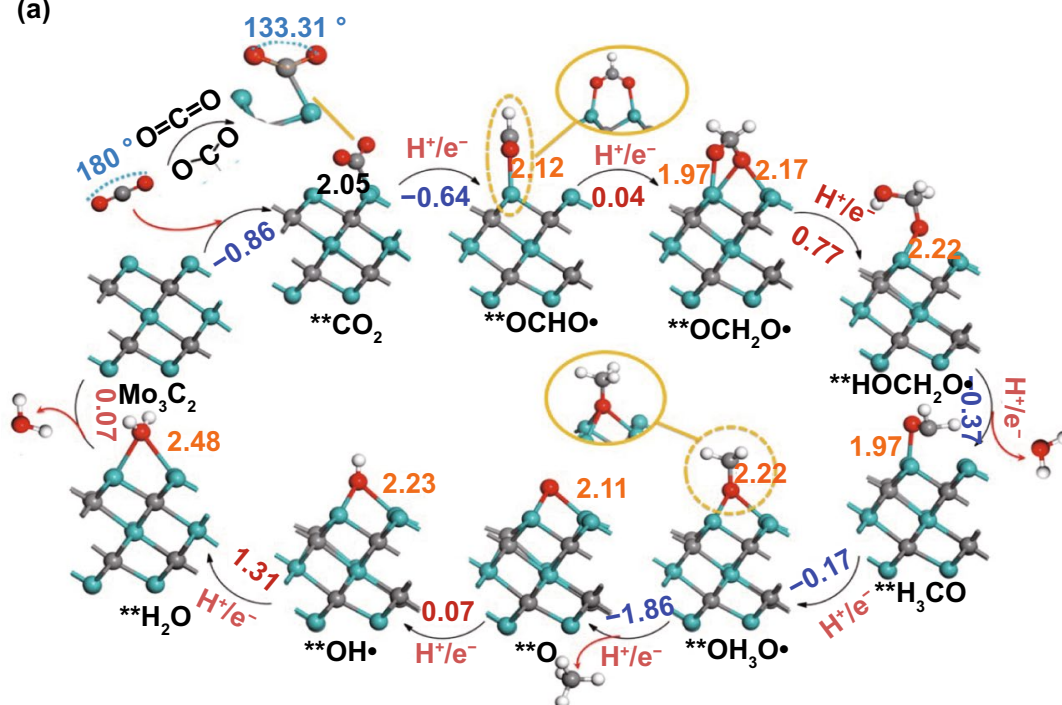

(b)
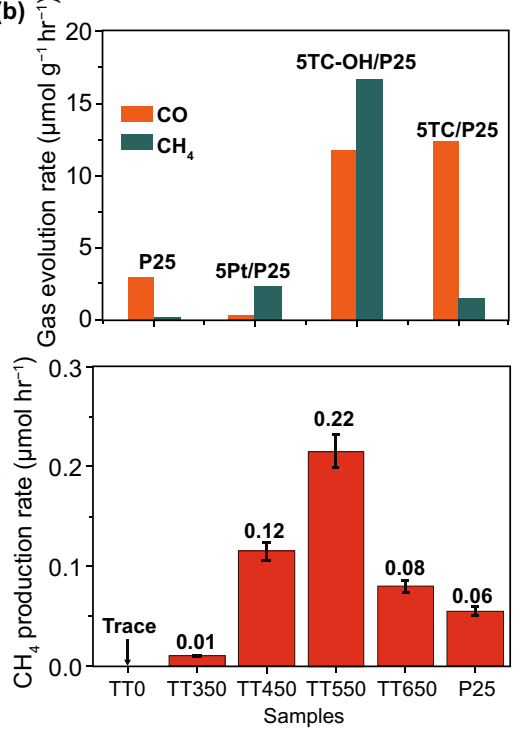

(e)

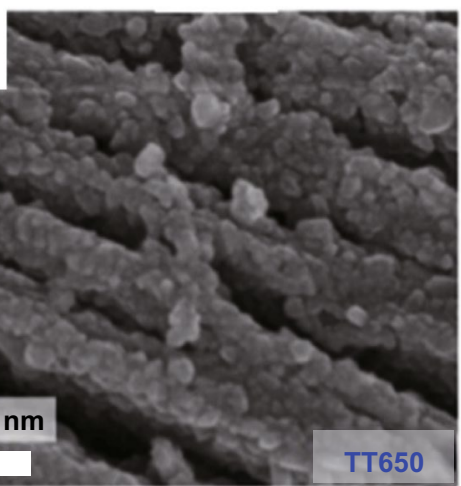

(f)

(c)

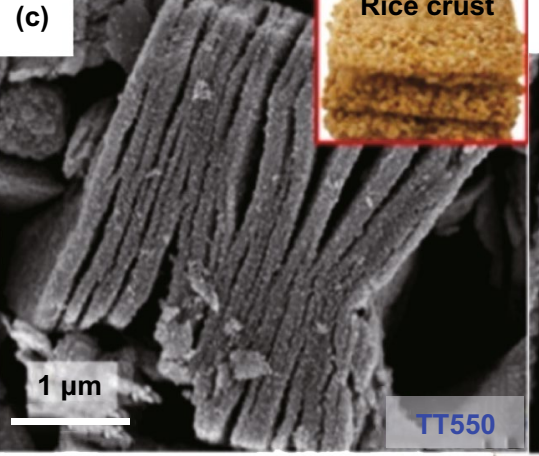

(d)
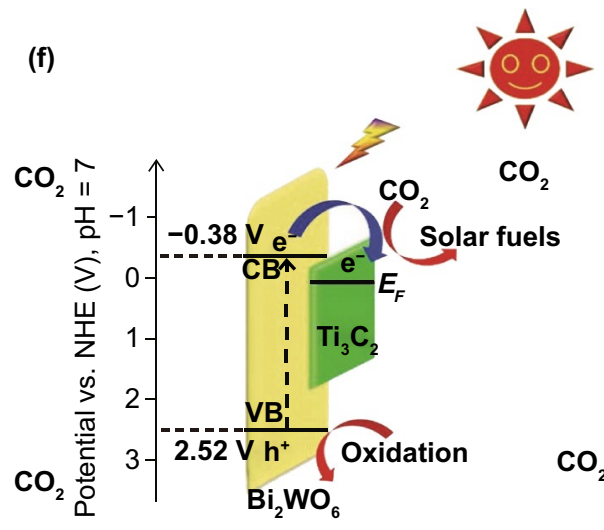

(g)

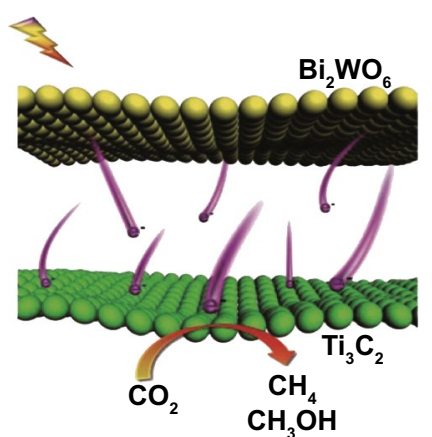

(h) I

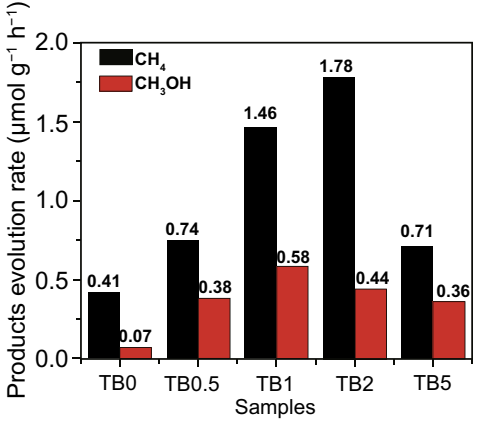

Fig. 20 a Lowest amount energy routes (PBE/DFT-D3 calculations) pursued for $\mathrm{CO}_{2}$ into $* \mathrm{CH}_{4}$ and $* * \mathrm{H}_{2} \mathrm{O}$ conversion, catalyzed by $\mathrm{Mo}_{3} \mathrm{C}_{2}$. Gray, lilac, red, and white spheres indicate $\mathrm{C}, \mathrm{Mo}, \mathrm{O}$, and $\mathrm{H}$ atoms, correspondingly (Li et al. [248]). b Photo-catalytic $\mathrm{CO}$ and $\mathrm{CH}_{4}$ evolution rates over P25, 5Pt/P25, 5TC/P25, and 5TC-OH/P25 (Ye et al. [249]). C-f Photo-catalytic $\mathrm{CO}_{2} \mathrm{RR}$ of the $\mathrm{TiO}_{2} / \mathrm{Ti}_{3} \mathrm{C}_{2}(\mathrm{TT}-x$ ) samples and P25 for

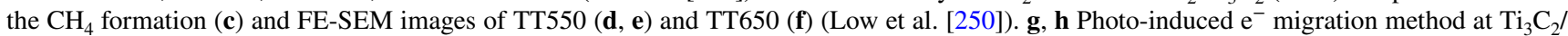
$\mathrm{Bi}_{2} \mathrm{WO}_{6}$ hetero-interface (g) and photo-catalytic activity of $\mathrm{Ti}_{3} \mathrm{C}_{2} / \mathrm{Bi}_{2} \mathrm{WO}_{6}$ through diverse mass ratios of $\mathrm{Ti}_{3} \mathrm{C}_{2}$ to $\mathrm{Bi}_{2} \mathrm{WO}_{6}(0 \%, 0.5 \%, 1 \%, 2 \%$, and 5\%) (h). Adapted with permission from Ref. [251] 
separation and efficiently boosted carrier lifetime that permits more $\mathrm{e}^{-} \mathrm{s}$ to engage in $\mathrm{CO}_{2}$ photo-reduction. To get benefit from already discussed benefits, $\mathrm{V}_{\mathrm{v}}$-rich o-BiVO showed improved conversion effectiveness with methanol production rate of $398.3 \mu \mathrm{mol} \mathrm{g}^{-1} \mathrm{~h}^{-1}$. In photo-reduction method, just small concentration of $\mathrm{H}_{2}$ and trace amount of ethanol can be detected, signifying highly suitable method for product selection. Furthermore, the $\mathrm{V}_{\mathrm{v}}$-rich o- $\mathrm{BiVO}_{4}$ can undergo continuous photo-reduction reaction up to $96 \mathrm{~h}$, lacking any clear decrease in the photo-catalytic efficiency. The above such outcomes show that the $2 \mathrm{D}$ photo-catalysts are efficient choice for getting highly efficient $\mathrm{CO}_{2}$ reduction. In case of pristine MXene, somehow the consideration of surface terminations ( $-\mathrm{OH}$ and $-\mathrm{O}$ functional group) was very important to clarify reaction steps for $\mathrm{CO}_{2} \mathrm{RR}$. In case of the $\mathrm{Cr}_{3} \mathrm{C}_{2}$ and $\mathrm{Mo}_{3} \mathrm{C}_{2}$ MXene, with no surface termination groups, energy input of 1.05 and $1.31 \mathrm{eV}$ was essential for transfer of $\mathrm{CO}_{2}$ to $\mathrm{CH}_{4}$ (Fig. 20a). While $\mathrm{Mo}_{3} \mathrm{C}_{2}$ surface was terminated with $\mathrm{OH}$ or $\mathrm{O}$, energy was further decreased to 0.35 and $0.54 \mathrm{eV}$, correspondingly. Therefore, $\mathrm{OH}$ or O-terminated MXene was certainly made easy $\mathrm{CO}_{2}$ conversion in comparison with un-functionalized MXene. Moreover, photo-catalytic reduction of $\mathrm{CO}_{2}$ at $\mathrm{V}_{\mathrm{o}}$ on $\mathrm{Ti}_{2} \mathrm{CO}_{2}, \mathrm{Ti}_{3} \mathrm{C}_{2} \mathrm{O}_{2}$, and $\mathrm{V}_{2} \mathrm{CO}_{2}$ was studied theoretically by the first-principles DFT simulations [247]. These results showed that $\mathrm{Ti}_{2} \mathrm{CO}_{2}$ needs the minimum energy of reaction method and therefore proves good $\mathrm{CO}_{2} \mathrm{RR}$ activity. The $\mathrm{CO}_{2}$ was decreased to subsequent four compounds: $\mathrm{HCHO}, \mathrm{CH}_{3} \mathrm{OH}, \mathrm{CH}_{4}$, and $\mathrm{HCOOH}$. Obviously, whole energy barrier for $\mathrm{CO}_{2}$ hydrogenation into $\mathrm{HCOOH}$ at $\mathrm{O}_{\mathrm{v}}$ in $\mathrm{Ti}_{2} \mathrm{CO}_{2} \mathrm{SL}$ was very good with just $0.53 \mathrm{eV}$ compared with other reduction products that needed superior kinetics. For example, $\mathrm{O}_{\mathrm{v}}$ on O-terminated MXene was active site in $\mathrm{CO}_{2} \mathrm{RR}$ for high $\mathrm{HCOOH}$ selectivity; therefore, MXene NSs can be used for broad range of applications.

Ye et al. [252] joined surface-alkalinized $\mathrm{Ti}_{3} \mathrm{C}_{2}$ MXene as co-catalysts with marketable $\mathrm{P} 25$ through a simple mechanical mixing technique for important improvement in photocatalytic $\mathrm{CO}_{2} \mathrm{RR}$. Following surface alkalinization, $5 \mathrm{wt} \%$ $\mathrm{Ti}_{3} \mathrm{C}_{2}(\mathrm{OH})_{2}$-doped P25 (5TC-OH/P25) reveals obvious support in $\mathrm{CH}_{4}$ release as compared to un-modified 5TC/P25 (Fig. 20b). The DFT study showed that adsorption energy of $\mathrm{CO}_{2}$ on TC-F (F-termination) was superior to $\mathrm{CO}_{2}$ on TC-OH (OH-termination). Thus, $\mathrm{CO}_{2}$ molecules were easily adsorbed at $\mathrm{TC}-\mathrm{OH}$ surface, leading to the synthesis of activated $\mathrm{CO}_{3}{ }^{2-}$. So, encouraging charge separation, extraordinary electrical conductance, sufficient $\mathrm{CO}_{2}$ adsorption, and activation sites on alkalinized MXene were main things contributed to photo-catalytic improvement. These clearly showed the major job of surface alkalinization of MXene, as a valuable metal-free co-catalyst for synthetic photo-synthesis.

Similarly, Low et al. [253] studied in situ formed $\mathrm{TiO}_{2}$ NPs on conductive $\mathrm{Ti}_{3} \mathrm{C}_{2}$, to form $\mathrm{TiO}_{2} / \mathrm{Ti}_{3} \mathrm{C}_{2}$ hybrids (TT- $x$, where $x$ shows the calcination temperature) through thermal annealing for $\mathrm{CH}_{4}$ production from $\mathrm{CO}_{2} \mathrm{RR}$ (Fig. 20c). After $-\mathrm{F}$ functional groups removal at elevated temperature, it brings oxidation of $\mathrm{Ti}_{3} \mathrm{C}_{2}$ tuned with $-\mathrm{O}$ functional groups. The TT550 and TT650 morphology was clearly different from $\mathrm{Ti}_{3} \mathrm{C}_{2}$ but analogous to rice crust (Fig. 20d-f). High conductivity of $\mathrm{Ti}_{3} \mathrm{C}_{2}$ promotes $\mathrm{e}^{-}$-transfer from $\mathrm{TiO}_{2}$ and exclusive rice crust analogue morphology with a large active site density, considerably push photo-catalytic activity. Referring to precede work which is discussed already [252], it was hard to straight evaluate both $\mathrm{TiO}_{2} / \mathrm{Ti}_{3} \mathrm{C}_{2} \mathrm{~T}_{x}$ photo-catalytic systems due to different synthesis methods, unlike $\mathrm{TiO}_{2}$ phase, discrete difference in morphology, and diverse MXene surface modification. Therefore, it is sensible to embrace AQY for $\mathrm{CO}_{2} \mathrm{RR}$ as a controlled tool to describe different future experiment conditions. In recent times, same group of researchers has formed ultra-thin $2 \mathrm{D} / 2 \mathrm{D} \mathrm{Ti}{ }_{3} \mathrm{C}_{2} /$ $\mathrm{Bi}_{2} \mathrm{WO}_{6}$ hetero-junction hybrid nano-composites (Fig. 20g) [254]. Due to electronic coupling and intense physical effects, 2D/2D hetero-junction noticeably improves transfer and partition of photo-induced charge carriers to reduce charge recombination. $2 \mathrm{wt} \% \mathrm{Ti}_{3} \mathrm{C}_{2}$-modified $\mathrm{Bi}_{2} \mathrm{WO}_{6} \mathrm{NSs}$ (NSs) $\left(\mathrm{TB}_{2}\right)$ records the highest $\mathrm{CH}_{4}$ release rate than other stoichiometry (Fig. 20h). Moreover, large interfacial contact surfaces of intimate 2D/2D hetero-junction donate more quick charge mobility in comparison with $0 \mathrm{D} / 2 \mathrm{D}$ and $1 \mathrm{D} / 2 \mathrm{D}$ hetero-junctions due to a decrease in charge transfer path. So, such stimulating investigation on parallel 2D/2D hetero-interfaces generates novel potential in material science for layered hetero-junctions design in electrocatalysis and photo-catalysis for energy conversion.

\subsection{Nitrogen $\left(\mathrm{N}_{2}\right)$ Fixation}

In comparison with $\mathrm{CO}_{2} \mathrm{RR}$, the photo-catalytic $\mathrm{N}_{2}$-fixation is still more demanding as dissociation enthalpy of $\mathrm{N}_{2}$ molecule triple bond $\left(962 \mathrm{~kJ} \mathrm{~mol}^{-1}\right)$. Normally, catalyst 
conversion for $\mathrm{N}_{2}$ is enormously harsh as $\mathrm{N}_{2}$ just weakly binds with solid-state catalysts and reaction entails highenergy intermediates. So, it is very much required to do an appropriate structure to support conversion competency of $\mathrm{N}_{2}$ to $\mathrm{NH}_{3}$. E0 to make the $\mathrm{N}^{2-}$ is as high as $-4.2 \mathrm{~V}$ versus NHE via $\mathrm{N}_{2}+\mathrm{e}^{-} \rightarrow \mathrm{N}^{2-}$ method, whereas proton-coupled $\mathrm{e}^{-}$transfer reaction $\mathrm{N}_{2}+\mathrm{H}^{+}+\mathrm{e}^{-} \rightarrow \mathrm{N}_{2} \mathrm{H}$ has more available $E_{0}$ of $-3.2 \mathrm{~V}$ versus NHE [255]. Proton-supported exchange method might evade production of high-energy intermediates and therefore reduce thermodynamic kinetics for $\mathrm{NH}_{3}$ formation. Current investigations showed that 2DMs are a talented applicant to get an efficient photo-catalytic $\mathrm{N}_{2}$ fixation [249]. Zhang and co-workers [250] studied that $\mathrm{V}_{\mathrm{o}}$ formation in $\mathrm{BiOBr}$ NSs can efficiently boost $\mathrm{N}_{2}$ fixation reaction. Theoretical study showed that $\mathrm{N}_{2}$ adsorption onto the $\mathrm{V}_{\mathrm{o}}$ through an end on arrangement of adsorbed $\mathrm{N}_{2}$ triple bond can be extended from $1.078 \AA$ in original $\mathrm{N}_{2}$ to $1.133 \AA$, signifying efficient $\mathrm{N}_{2}$ activation. Generated $\mathrm{e}^{-} \mathrm{s}$ in BiOBr can simply inject into $\mathrm{N}_{2} \pi$ antibonding orbitals. Accessibility of localized $\pi$-back donating $\mathrm{e}^{-} \mathrm{s}$ in $\mathrm{V}_{\mathrm{o}}$ might efficiently adsorb $\mathrm{N}_{2}$ to generate activation. So, $\mathrm{N}_{2}$ reduction to $\mathrm{NH}_{3}$ catalyzed through the $\mathrm{BiOBr}$ with $\mathrm{V}_{\mathrm{o}}$ needs a very low reaction kinetic and a higher photo-catalytic performance can be obtained. $\mathrm{N}_{2}$ conversion rate after vis-light and UV-Vis-light irradiation is 104.2 and $223.3 \mu \mathrm{mol} \mathrm{g}{ }^{-1} \mathrm{~h}^{-1}$, respectively, after without $\mathrm{h}^{+}$scavenger or co-catalyst. Stimulated through $\mathrm{V}_{\mathrm{o}}$-motivated $\mathrm{N}_{2}$ activation, $\mathrm{MoS}_{2} \mathrm{NSs}$ with $\mathrm{S}$-vacancy were formed and further utilized to $\mathrm{N}_{2}$ fixation. The $\mathrm{MoS}_{2} \mathrm{NSs}$ showed $\mathrm{NH}_{3}$ formation rate almost $325 \mu \mathrm{mol} \mathrm{g}{ }^{-1}$ with 10-h measurement after simulated solar light irradiation. Although marketable bulk $\mathrm{MoS}_{2}$ cannot give any photo-catalytic performance for $\mathrm{NH}_{3}$ synthesis under similar test condition, it illustrates exclusive benefit of $\mathrm{MoS}_{2} \mathrm{NSs}$ for $\mathrm{N}_{2}$-reduction. According to Mott-Schottky spectra, $\mathrm{MoS}_{2} \mathrm{NSs}$ and bulk $\mathrm{MoS}_{2}$ samples in CB positions were anticipated to be -0.35 and $-0.24 \mathrm{~V}$, correspondingly, that were positioned below thermodynamic reduction potentials of $\mathrm{N}_{2}$ through one or two-e ${ }^{-}$transfer method. So, it was presumed that $\mathrm{N}_{2}$ reduction with $\mathrm{MoS}_{2}$ NSs was multielectron coupled proton transfer method. Due to n-type SC essence, there subsists many free $\mathrm{e}^{-} \mathrm{s}$ in $\mathrm{MoS}_{2}$ NSs and such free $\mathrm{e}^{-} \mathrm{s}$ can pair with photo-generated excitons to form charge excitons (trions) that were mostly located around Mo-sites. Produced trions have manifold $\mathrm{e}^{-} \mathrm{s}$ in one bound state that was useful to multi- $\mathrm{e}^{-}$migration reactions. While $\mathrm{N}_{2}$ is confined through $\mathrm{S}$-vacancies, it was bounded via three
Mo atoms with trions after irradiation. $\mathrm{N}_{2}$ activated after $\mathrm{e}^{-} \mathrm{s}$ donation from bonding orbitals and accepting $\mathrm{e}^{-} \mathrm{s}$ to its antibonding orbitals results in a trion-supported six-electron reduction method. The significant photo-catalytic $\mathrm{N}_{2}$ fixation resides in building adsorption site in $\mathrm{N}_{2}$ molecule, $\mathrm{e}^{-}$-rich systems for $\mathrm{e}^{-}$donation, engineering band configuration through enough $\mathrm{E}_{0}$ and coupling protons to decrease energy condition of intermediates. So, theoretical and experimental study showed that novel breakthrough on MXene-based composites for $\mathrm{CO}_{2} \mathrm{RR}$ and $\mathrm{N}_{2} \mathrm{RR}$ will appear in the frontline of technology and science, transferring them in future real energy applications.

\subsection{Organic Synthesis}

Idea of decreasing energy expenditure for chemically developed, solar light-driven chemical transformation through assist of the SCs holds huge view. Under irradiation, SCs can use solar light to make an exciton or hot carriers that can stimulate chemical reactions at surface of catalyst. Effectiveness and selectivity of the photo-catalytic conversion are still in adequate at large scale. Weak interaction of $\mathrm{O}_{2}$-molecule with photo-catalysts surfaces, particularly a defect-free surface, is a serious matter for poor effectiveness of photo-catalytic organic formations. Reaction involves $\mathrm{O}_{2}$, which needs efficient interfacial $\mathrm{e}^{-}$-transfer, either directly as donor or indirectly as $\mathrm{e}^{-}$-acceptor [256]. Additional significant subject is poor selectivity that might be obtained from photo-generated $\mathrm{h}^{+} \mathrm{s}$. Generally, generated $\mathrm{h}^{+} \mathrm{s}$ has strong oxidizing capacity and is accountable for nonselective overoxidation. In recent times, the 2D photo-catalysts displayed a huge promise for selective organic transformation using mild conditions. For example, Xiong et al. [257] formed $\mathrm{V}_{\mathrm{o}}$ into ultra-thin $\mathrm{WO}_{3} \mathrm{NSs}$ to make $\mathrm{O}_{2}$-molecules active and activate organic conversion. Defect-rich (DR) $\mathrm{WO}_{3}$ NSs were formed through calcination of the first synthesized $\mathrm{WO}_{3} \cdot \mathrm{H}_{2} \mathrm{O} \mathrm{NSs}$ in $\mathrm{N}_{2}$-environment, at $673 \mathrm{~K}$, whereas defect-deficient (DD) $\mathrm{WO}_{3}$ NSs were synthesized via calcination in atmospheric condition. From aberration-corrected HAADF-STEM, it is clear that $\mathrm{DD} \mathrm{WO}_{3}$ shows comparatively smooth and flat surface. Simultaneously, continuous and controlled lattice fringe was experienced from atomic resolution HAADF-STEM image (Fig. 21). Such outcomes conclusively proposed the defects deficiency in such DD $\mathrm{WO}_{3}$ sample. In $\mathrm{DR} \mathrm{WO}_{3}$, many small pits were formed, 

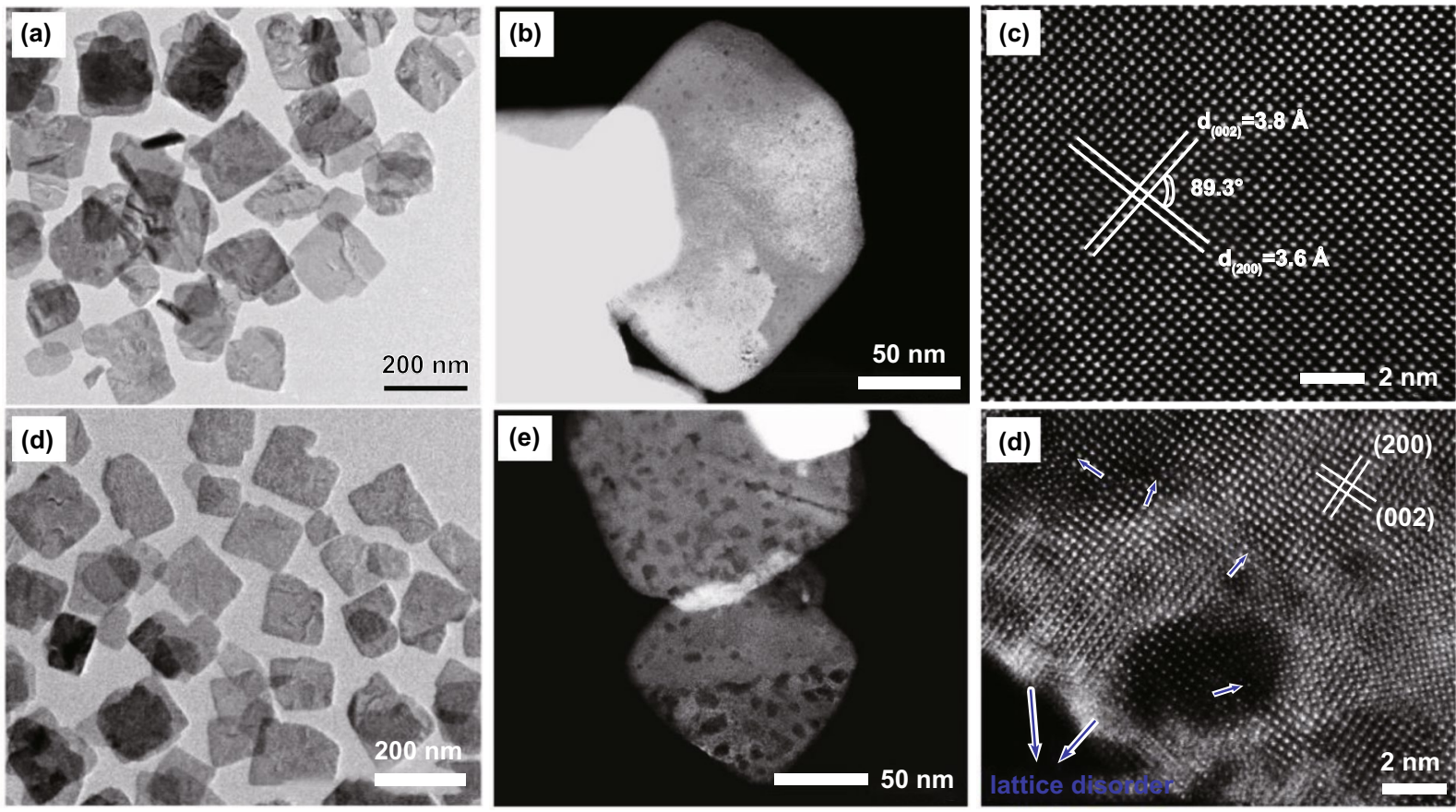

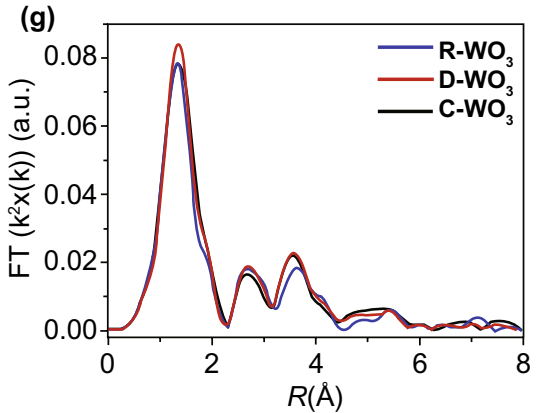

(j)

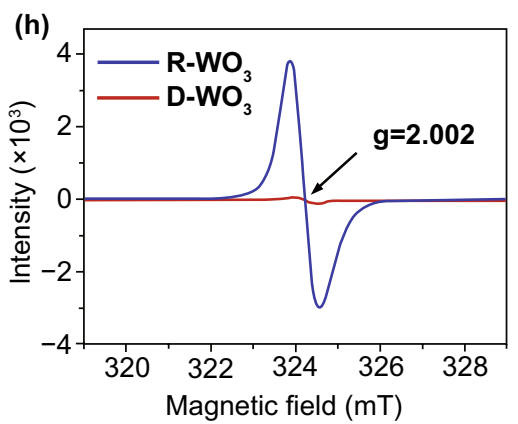

(k) (i)

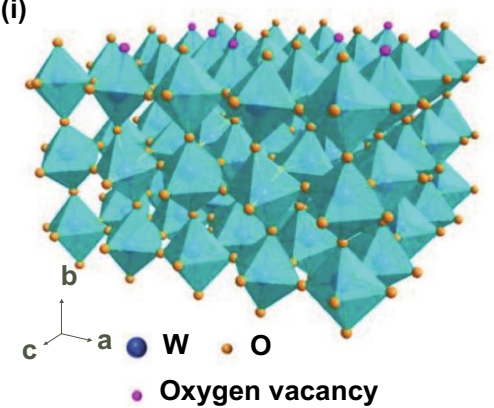

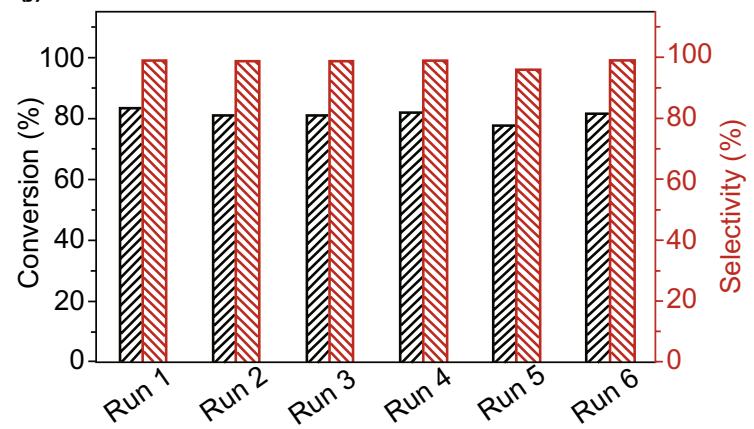

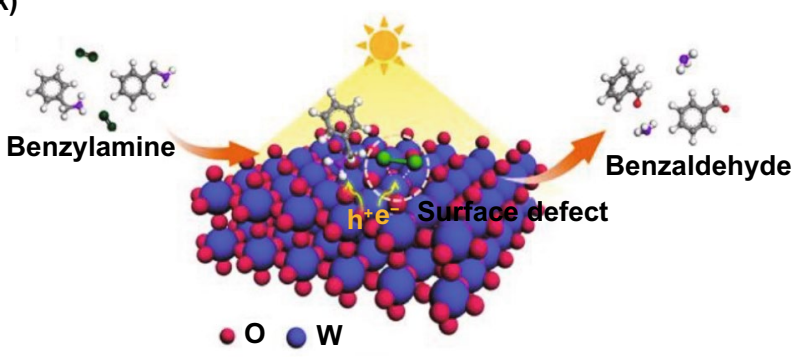

Defect-rich $\mathrm{WO}_{3}$ nanosheet

Fig. 21 a-f Morphological study. g Fourier transforms W L3-edge EXAFS spectra with respect to commercial $\mathrm{WO}_{3}$. h ESR spectra at room temperature. i Scheme showing locations of $\mathrm{V}_{\mathrm{o}}$ in $\mathrm{WO}_{3}$ lattice. $\mathbf{j}$ Cyclic analysis for defect-rich $\mathrm{WO}_{3}$ in catalytic aerobic coupling of benzylamine after irradiation with $\lambda>400 \mathrm{~nm}$ at $298 \mathrm{~K}$. k Scheme shows total light-driven catalytic reaction route. Adapted with permission from Ref. [257]

with small lattice disorder, and dislocation appears in NSs that vigorously emphasize the presence of different defects.

To further reveal the presence of $\mathrm{V}_{\mathrm{o}}$, synchrotron radiation-related XAFS spectroscopy was performed. The DR $\mathrm{WO}_{3}$ showed a clear diverse confined atomic configuration compared to $\mathrm{DD} \mathrm{WO}_{3}$, where $\mathrm{W}$-atom coordination num-

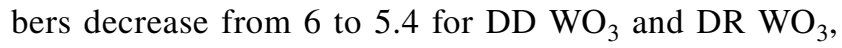
showing local deficiency of $\mathrm{O}_{2}$-atom. ESR investigation was also used to calculate $\mathrm{WO}_{3}$ samples. Noticeably, at $g=2.002$ a symmetric ESR signal was experimented for 
the DR $\mathrm{WO}_{3}$, showing $\mathrm{e}^{-}$-entrapping at $\mathrm{V}_{\mathrm{o}}$. Joint STEM, XAFS, and ESR findings suggested that subsistence of $\mathrm{V}_{\mathrm{o}}$ at precise sites induces few lattice distortion and displacement. After getting benefits from DR configuration, $\mathrm{O}_{2}$ was chemisorbed at $\mathrm{V}_{\mathrm{o}}$ of defect-rich $\mathrm{WO}_{3} \mathrm{NSs}$ through end on arrangement and associated with e-s transfer from coordinative unsaturated site to $\mathrm{O}_{2}$. So, $\mathrm{O}_{2}$ was efficiently activated to $\mathrm{O}^{2 \cdot-}$ species over $\mathrm{DR} \mathrm{WO}_{3}$, and it was transformed from amines to respective imines with 6 times enhanced kinetic rate compared to $\mathrm{DD}_{\mathrm{WO}_{3}}$. As reaction time increased to $8 \mathrm{~h}$ as conversion ratio of benzylamine was greater compared to $80 \%$ with an extremely high selectivity, no clear decline was observed within 6 times of catalytic cycles. Other than $\mathrm{O}_{2}$-activation, hydrophobicity was another significant feature to affect photo-catalytic organic conversion method. Li and co-workers [258] studied colloidal formation approach information of $\mathrm{BiOCl} \mathrm{NSs}$. Through $\mathrm{BiCl}_{3}$ hydrolysis in octa-decylene solution, supported by in situ preparation of $\mathrm{H}_{2} \mathrm{O}$ via reaction in oleylamine and oleate solution, singlecrystalline BiOCl colloidal NSs (BiOCl C-UTNSs) were obtained with almost $3.7 \mathrm{~nm}$ thickness. For comparison, $\mathrm{BiOCl} \mathrm{NSs}$ were also formed through hydrothermal way, known as BiOCl H-UT-NSs. Surface $\mathrm{H}_{2} \mathrm{O}$ contact angle (CA) measurement was utilized to establish wettability of as-synthesized BiOCl NSs. BiOCl C-UT-NSs showed a $\mathrm{H}_{2} \mathrm{O} C A$ of $116.3^{\circ}$, which is hydrophobic. It occurs from detail that organic ligands have capped on $\mathrm{BiOCl} \mathrm{C-UT-}$ NSs surface during colloidal formation. Conversely, $\mathrm{BiOCl}$ H-UT-NSs exhibited a $\mathrm{H}_{2} \mathrm{O} C A$ of $0^{\circ}$, showing the superhydrophilic nature of synthesized BiOCl H-UT-NSs. Huge difference in BiOCl C-UT-NSs and BiOCl H-UT-NSs may bring about important effect for photo-catalytic organic conversion method. Moreover, there subsist plentiful $\mathrm{V}_{\mathrm{o}}$ on BiOCl C-UT-NSs, ensuing strong light absorption in vis-light range. To get benefit from hydrophobic character and enhanced light harvesting capability, BiOCl C-UT-NSs displayed greatly enhanced photo-catalytic activity for conversion of $\mathrm{N}$-t-butylbenzylamine to $\mathrm{N}$-t-butyl-benzylamine. The $78 \%$ conversion ratio was obtained from BiOCl C-UTNSs, whereas BiOCl H-UT-NSs have only displayed about $15 \%$ conversion rate, from Xe lamp irradiation for $1 \mathrm{~h}$. Furthermore, BiOCl C-UT-NS sample was more utilized for conversion of secondary amines to respective imines along increased conversion selectivity as well as efficiency. The 2D photo-catalysts were verified to be talented choice for the photo-catalytic organic formation, and such approach can broaden perceptive of organic conversion method and is favorable to establish further proficient organic transformation systems.

\subsection{Removal of Pollutants}

In the progress of financial and industrialization, environmental pollution is the main problem that threatens the public health. The photo-catalysis was considered as an efficient and financially viable technology to handle elimination of environmental pollutions. Due to exclusive advantages such as better adsorption capability of pollutants and strong light harvesting capacitance, 2DMs-based photo-catalysts showed a great hope for removal of pollutants. For instance, Xia and co-workers [140] formed ultra-thin $\mathrm{Bi}_{4} \mathrm{O}_{5} \mathrm{Br}_{2}$ NSs through reactive ionic liquid supported via solvothermal method in combination with $\mathrm{pH}$ adjustment. In ionic liquids, long carbon chain served as a capping reagent which controls the crystal growth along c-axis. Simultaneously, reaction condition $\mathrm{pH}$ was adjusted to 11 that supplied $\mathrm{OH}^{-}$to substitute $\mathrm{Br}$ - to execute de-halogenation in fabrication route of $\mathrm{Bi}_{4} \mathrm{O}_{5} \mathrm{Br}_{2}$. So, both thickness and component-engineered $\mathrm{Bi}_{4} \mathrm{O}_{5} \mathrm{Br}_{2}$ materials were formed and utilized in photo-catalytic degradation of an antibiotic CIP and tetracycline (TC) after irradiation with vis-light for $120 \mathrm{~min}$. After irradiation, $75 \%$ of CIP is photo-degraded through $\mathrm{Bi}_{4} \mathrm{O}_{5} \mathrm{Br}_{2}$, whereas $\mathrm{BiOBr}$ degradation rate was $51.4 \%$. Furthermore, $\mathrm{Bi}_{4} \mathrm{O}_{5} \mathrm{Br}_{2}$ NSs showed $77.8 \%$ degradation rate for TC within $60-$ min irradiation, which is very high compared to $\mathrm{BiOBr}$ with just $31.7 \%$. Changeable energy band configuration of $\mathrm{Bi}_{4} \mathrm{O}_{5} \mathrm{Br}_{2}$ was verified to consider the increased photo-catalytic activity. More negative $\mathrm{CB}$ position of $\mathrm{Bi}_{4} \mathrm{O}_{5} \mathrm{Br}_{2}$ will make easy development of more active $\mathrm{O}_{2}^{--}$species. Upshifting of $\mathrm{CB}$ position and broad VB will be advantageous to improve charge separation effectiveness. Thus, obtained $\mathrm{Bi}_{4} \mathrm{O}_{5} \mathrm{Br}_{2}$ NSs displayed greater performance toward pollutant removal. To further enhance photo-catalytic performance in pollutant degradation, creating surface defects might be another approach. Therefore, Xie and co-workers [119] studied that while $\mathrm{BiOCl}$ thickness is reduced from 30 to $2.7 \mathrm{~nm}$, in $\mathrm{BiOCl}$ defect type will vary from isolate defects $\mathrm{VBi}^{\prime \prime \prime}$ to triple vacancy-related $\mathrm{VBi}^{\prime \prime} \mathrm{VO} \mathrm{VBi}^{\prime \prime \prime}$, as confirmed with PAS. Through desirable quality from triple vacancy-associated $\mathrm{VBi}^{\prime \prime} \mathrm{VO} \mathrm{VBi}^{\prime \prime \prime}$ along four negative charges, $\mathrm{BiOCl}$ NSs were additionally negatively charged as comparative 

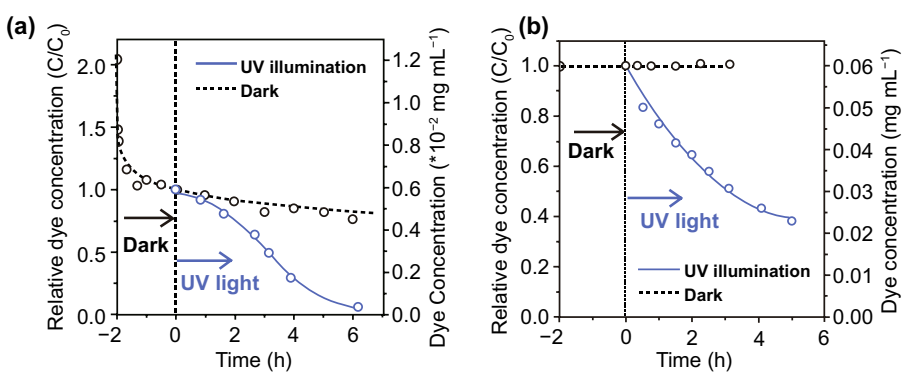

(c)

(d)
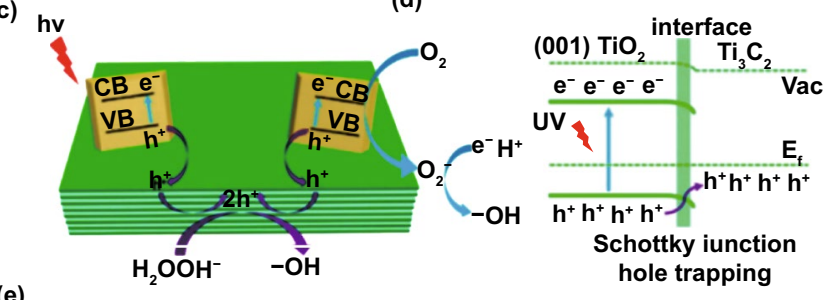

(e)
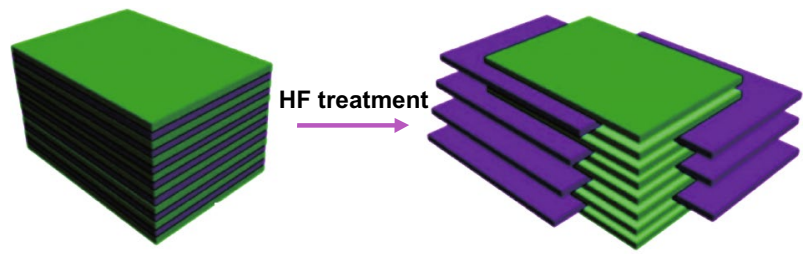

Raw $\mathrm{Ti}_{3} \mathrm{AlC}_{2}$

HF selectively exfoliates

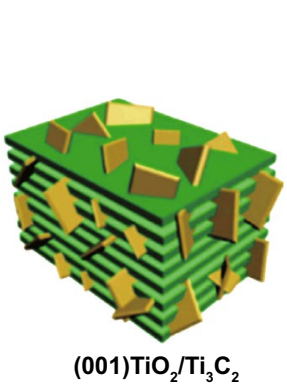
the Al layers from $\mathrm{Ti}_{3} \mathrm{AlC}_{2}$

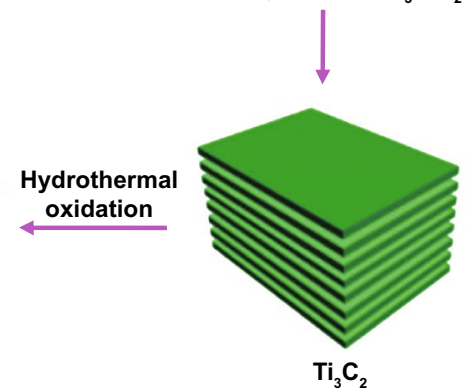

Fig. 22 Photo-catalytic pollutant degradation utilizing MXenes and their hybrid nano-composites. a, b Time-dependent MB respective level (a) and $\mathrm{AB} 80$ (b) in $\mathrm{Ti}_{3} \mathrm{C}_{2} \mathrm{~T}_{x}$. Adapted with permission from Ref. [135]). c-e Preparation of (001) $\mathrm{TiO}_{2} / \mathrm{Ti}_{3} \mathrm{C}_{2}$ nano-hybrids (c), charge transfer process (d), B.G. (e) of (001) $\mathrm{TiO}_{2} / \mathrm{Ti}_{3} \mathrm{C}_{2}$ after light irradiation. Adapted with permission from Ref. [259]

to $\mathrm{BiOCl}$ nano-plates. As $\mathrm{RhB}$ was positively charged, the further negatively charged ultra-thin $\mathrm{BiOCl}$ NSs promoted $\mathrm{RhB}$ adsorption on BiOCl NSs' surface. Moreover, the presence of vacancies can enhance light absorption and speed up separation of charges. As a result, ultra-thin BiOCl NSs exhibited a great solar photo-catalytic activity for $\mathrm{RhB}$ removal. MXene shows outstanding performance in photocatalytic degradation of organic pollutants. Mashtalir et al. [135] utilized $\mathrm{Ti}_{3} \mathrm{C}_{2} \mathrm{~T}_{x}$ to degrade $\mathrm{MB}$ (a cationic dye) and acid blue 80 (AB80) (an anionic dye) (Fig. 22a, b). The MB and $\mathrm{AB} 80$ degradation was augmented via UV irradiation.
In dark, $\mathrm{MB}$ level reduces due to negatively charged adsorption at $\mathrm{Ti}_{3} \mathrm{C}_{2} \mathrm{~T}_{x}$ surfaces with MB. After UV irradiation, a substantial reduction in $\mathrm{MB}$ and $\mathrm{AB} 80$ concentration, with $81 \%$ and $62 \%$, respectively, was experienced in existence of suspended $\mathrm{Ti}_{3} \mathrm{C}_{2} \mathrm{~T}_{x}$. It is observed that over longtime period, $\mathrm{Ti}_{3} \mathrm{C}_{2} \mathrm{~T}_{x}$ oxidation to form $\mathrm{TiO}_{2}$ in dissolved $\mathrm{O}_{2}$ presence was obvious that merits wide ranging research in this field. Similar to $\mathrm{G}_{-} \mathrm{TiO}_{2}$ nano-composites, it was imagined that $\mathrm{Ti}_{3} \mathrm{C}_{2} \mathrm{~T}_{x}$-supported $\mathrm{TiO}_{2}$ may function as a possible catalyst that further promotes progress of this direction. While continuing such investigation for the design of hetero-junction 
(a)

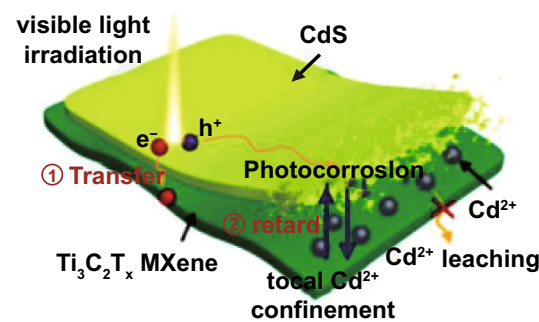

(d)

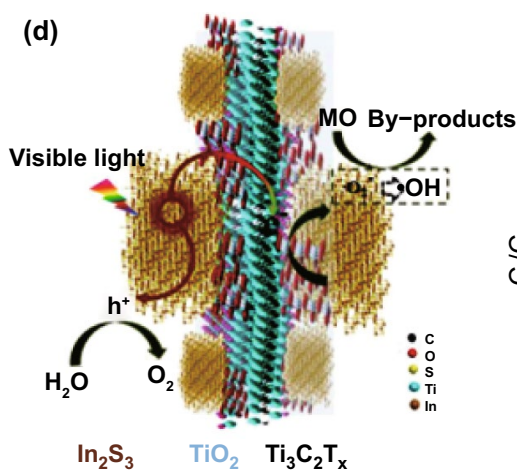

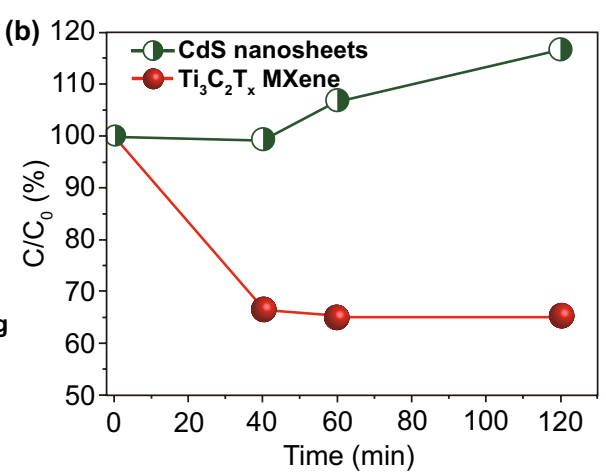
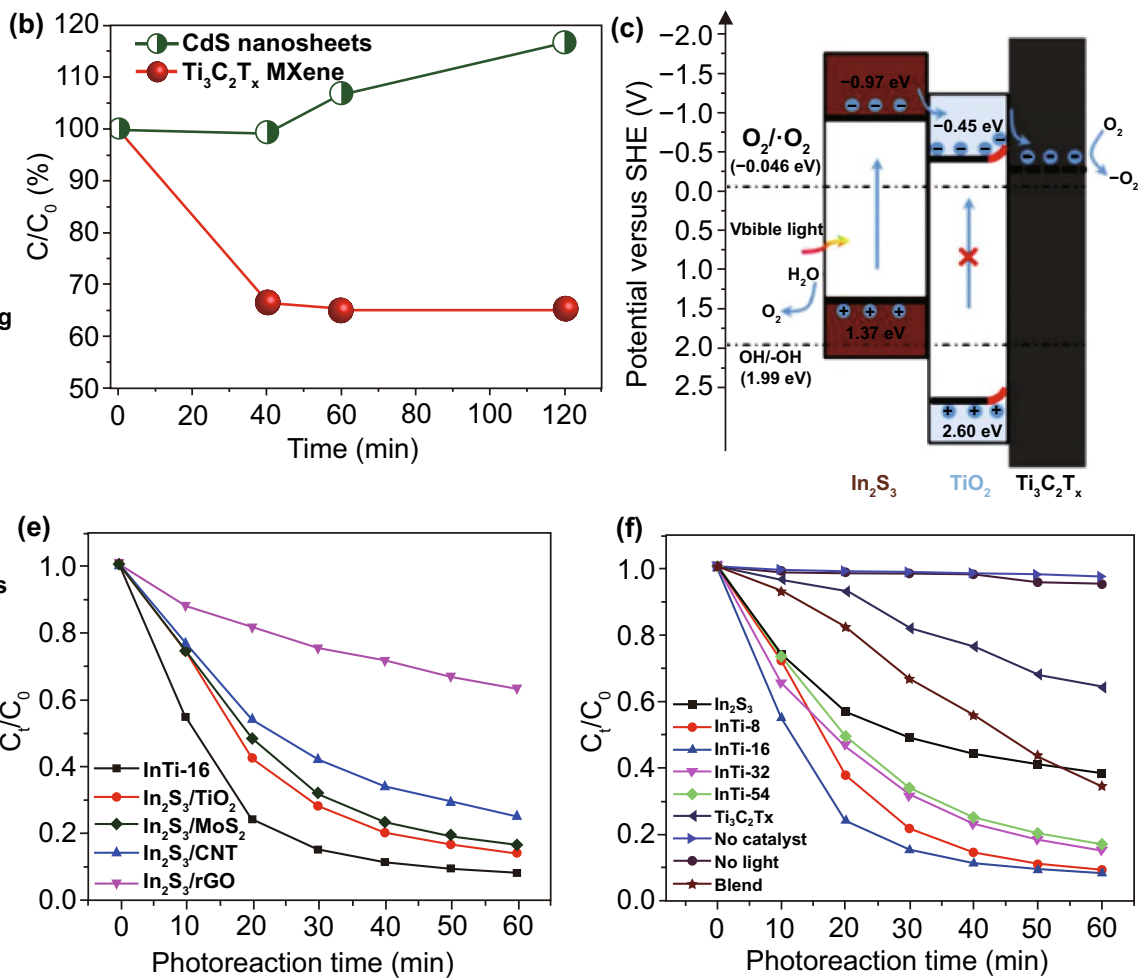

Fig. 23 a Scheme of CdS/Ti ${ }_{3} \mathrm{C}_{2} \mathrm{~T}_{x}$ NSs to improved photo-activity and photo-stability. b Adsorption of $\mathrm{Cd}^{2+}$ over CdS NSs and Ti ${ }_{3} \mathrm{C}_{2} \mathrm{~T}_{x} \mathrm{MXene}$ in dark. Adapted with permission from Ref. [261]. c Charge migration. d Separation and reaction mechanism for MO degradation in $\mathrm{In}_{2} \mathrm{~S}_{3} /$ anatase $\mathrm{TiO}_{2} @$ metallic $\mathrm{Ti}_{3} \mathrm{C}_{2} \mathrm{~T}_{x}$ ( $\mathrm{InTi}$ ) system after vis-light and photo-catalytic degradation of $\mathrm{MO}$ over other $\mathrm{In}_{2} \mathrm{~S}_{3}$-based binary hybrids. e $\mathrm{In}_{2} \mathrm{~S}_{3}$, In Ti hybrids, and $\mathrm{Ti}_{3} \mathrm{C}_{2} \mathrm{~T}_{x}$. f In Ti- $x$ indicates mass of $\mathrm{Ti}_{3} \mathrm{C}_{2} \mathrm{~T}_{x}$ included during preparation $(x=8,16,32$, and $54 \mathrm{mg})$. Adapted with permission from Ref. [262]

interfaces, Peng et al. [259] studied a composite of $\mathrm{Ti}_{3} \mathrm{C}_{2}$ and $\{001\}$ facets-exposed $\mathrm{TiO}_{2}$ through hydrothermal incomplete oxidation of $\mathrm{Ti}_{3} \mathrm{C}_{2}$ (Fig. 22c-e).

In photo-catalytic reaction, $\mathrm{TiO}_{2}$ through preferential $\{001\}$ facets creates $\mathrm{e}^{-} \mathrm{s}$ and $\mathrm{h}^{+} \mathrm{s}$ through UV light illumination and, afterward, $\mathrm{Ti}_{3} \mathrm{C}_{2}$ develops a Schottky junction with $\{001\}$-face $\mathrm{n}$-type $\mathrm{TiO}_{2}$. It hinders recombination of $\mathrm{e}^{-} \mathrm{s}$ with $\mathrm{h}^{+} \mathrm{s}$ due to $\mathrm{SB}$. Enriched $\mathrm{e}^{-} \mathrm{s}$ on $\mathrm{TiO}_{2}(001)$ might interact with dissolved $\mathrm{O}_{2}$ to generate superoxide radical anions $\left(\mathrm{O}^{2-}\right)$ that further respond to $\mathrm{H}^{+}$and $\mathrm{e}^{-}$to produce extremely reactive hydroxyl $(\mathrm{OH})$ radicals, which increase photo-degradation. It is well recognized that photo-catalytic activity of $\mathrm{TiO}_{2}$ relies not only on particle shape but also on its exposed facets. It seems that $\{001\}$ surface offers oxidation sites in photo-catalytic method, while $\{101\}$ facets proceed as reductive sites. Therefore, $\mathrm{TiO}_{2}$ facet tuning using MXene has basic significance to systematically untangle underlying photo-catalytic system [241]. In addition, $\mathrm{TiO}_{2}$ metal sulfides are also utilized to join with $\mathrm{Ti}_{3} \mathrm{C}_{2} \mathrm{~T}_{x}$. Xie et al. [260] formed a $2 \mathrm{D}$ inplane $\mathrm{CdS} / \mathrm{Ti}_{3} \mathrm{C}_{2} \mathrm{~T}_{x}$ onto sheet hetero-structures via electrostatic self-assembly method (Fig. 23a). In such catalytic system, the $\mathrm{Ti}_{3} \mathrm{C}_{2} \mathrm{~T}_{x}$ Janus co-catalysts not just act as an $\mathrm{e}^{-}$mediator to augment $\mathrm{e}^{-} \mathrm{s}$ extraction from CdS but also restrain $\mathrm{h}^{+}$-mediated photo-corrosion of CdS. Assigning $\mathrm{Ti}_{3} \mathrm{C}_{2} \mathrm{~T}_{x}$ small Fermi level than CdS CB, photo-e ${ }^{-}$lifetime of $\mathrm{CdS} / 0.5 \% \mathrm{Ti}_{3} \mathrm{C}_{2} \mathrm{~T}_{x}$ was longer as compared to bare $\mathrm{CdS}$.

Moreover, $\mathrm{Ti}_{3} \mathrm{C}_{2} \mathrm{~T}_{x}$ can absorb $\mathrm{Cd}^{2+}$ ions that were produced during photo-catalysis (Fig. 23b) and, as a result, avoid $\mathrm{Cd}^{2+}$-ions dissolution in $\mathrm{H}_{2} \mathrm{O}$ to enhance photo-stability of CdS. For instance, double-gain strategy offers a conceptual idea to evade instability as well as photo-corrosion of CdS. Other than MXene-based binary hybrid nano-composites, prosperous study into ternary hetero-structures has become a mainstream in photo-catalysis area. Wang et al. [262] (2018) formed a new quasi-core-shell $\mathrm{In}_{2} \mathrm{~S}_{3}$ /anatase $\mathrm{TiO}_{2} @$ metallic $\mathrm{Ti}_{3} \mathrm{C}_{2} \mathrm{~T}_{x}$ MXene hetero-structure hybrids through in situ hydrothermal technique for degradation improvement in 
methyl orange (MO). Fascinatingly, enhanced photo-activity of ternary nano-architectures was credited to numerous associated factors, such as well-designed type II band arrangement and noble metal-free-based Schottky junction with promising charge migrating channels (Fig. 23c, d). Specially, such occurrence originates from synergistic contribution in between vis-light-responsive $\operatorname{In}_{2} \mathrm{~S}_{3}$, upward band bending in $\mathrm{TiO}_{2}$, and amazing electrical $\mathrm{Ti}_{3} \mathrm{C}_{2} \mathrm{~T}_{x}$ conductance. For example, optimal photo-catalyst in the presence of $\mathrm{Ti}_{3} \mathrm{C}_{2} \mathrm{~T}_{x}$ content of $16 \mathrm{mg}$ (InTi-16) evidents the highest photo-degradation competency for MO elimination, as well as more significantly, it exceeds other $\operatorname{In}_{2} \mathrm{~S}_{3}$-based binary NSs (Fig. 23e, f). Progressing via this analysis, it can be elucidated that key to ameliorate dyes photo-catalytic degradation is to effectively separate photo-induced $\left(\mathrm{e}^{-}-\mathrm{h}^{+}\right)$-pairs to hold back charge recombination. Consequently, efficiently tuned vigorous hetero-structure system with distinct quality (e.g., intimate contacted hetero-interfaces, use broad solar spectrum to imitate natural sunlight, superior conductance, constructive charge transfer, and separation) is an urgent demand for boosting photo-catalytic degradation. Incidentally, extensive research in chemistry, materials arrangement, and optimization is the main requirement. In turn, it will open new prospects for separating charge carrier dynamics in synergistically speeded up photo-activity in practical photo-catalytic applications.

\subsection{Hydrogen Peroxide $\left(\mathrm{H}_{2} \mathrm{O}_{2}\right)$ Production}

Since the first synthesis of $\mathrm{H}_{2} \mathrm{O}_{2}$ by Thenard (1818) as a result of barium peroxide reaction with nitric acid [263], $\mathrm{H}_{2} \mathrm{O}_{2}$ has gotten rising consideration in past 200 years due to which it was listed among the 100 most significant chemicals in world [264]. The anthraquinone oxidation (AQ) is mostly developed for $\mathrm{H}_{2} \mathrm{O}_{2}$ manufacture on industry level that is presently caused about $95 \%$ of total $\mathrm{H}_{2} \mathrm{O}_{2}$ formation. Normally, AQ method generally contains four steps [265]:

1. Hydrogenation of $\mathrm{AQ}$ in organic solvent by means of $\mathrm{Ni} /$ Pd catalyst.

2. Oxidation of hydrogenized AQ (HAQ) in air or $\mathrm{O}_{2}$-enriched atmosphere with the help of catalysts.

3. Removal of $\mathrm{H}_{2} \mathrm{O}_{2}$ and recycling $\mathrm{HAQ}$ to AQ.

4. Refinement and concentration of $\mathrm{H}_{2} \mathrm{O}_{2}$.
The multi-step oxidation and hydrogenation response need an elevated applied energy. On the other hand, AO method is not environmentally benevolent, since large quantity of waste $\mathrm{H}_{2} \mathrm{O}$ (for example, 2-ethyl-anthraquinone, tri-octyl phosphate, tert-butyl urea, and $\mathrm{K}_{2} \mathrm{CO}_{3}$ lye), exhaust gas (mesitylene isomers), and solid waste (activated alumina) was formed. It is well known that $\mathrm{H}_{2} \mathrm{O}_{2}$ is a very competent and ecological oxidant. It has maximum content of active $\mathrm{O}_{2}(47.1 \% \mathrm{w} / \mathrm{w})$, and no noxious side products are formed in its reactions, apart from $\mathrm{H}_{2} \mathrm{O}$ and $\mathrm{O}_{2}$. Due to these qualities, $\mathrm{H}_{2} \mathrm{O}_{2}$ has broadly applied in organic synthesis, [266] waste $\mathrm{H}_{2} \mathrm{O}$ management, disinfection [267], and paper industry [268]. Nowadays, $\mathrm{H}_{2} \mathrm{O}_{2}$ is studied in energy field as both oxidant and reductant in innovative fuel cell [269]. The results illustrated that theoretical output potential of the $\mathrm{H}_{2} \mathrm{O}_{2}$ fuel cell was $1.09 \mathrm{~V}$ that is analogous to traditional $\mathrm{H}_{2}$-fuel cell $(1.23 \mathrm{~V})$. The $\mathrm{H}_{2} \mathrm{O}_{2}$ has established rising concentration as it is not only a mild and environment friendly oxidant but also a talented novel liquid fuel. Formation of $\mathrm{H}_{2} \mathrm{O}_{2}$ by photocatalysis is green, sustainable, and potential method, in view of its utilization in $\mathrm{H}_{2} \mathrm{O}$ and $\mathrm{O}_{2}$ as source materials and solar light as energy. Other advantage of $\mathrm{H}_{2} \mathrm{O}_{2}$ as compared to $\mathrm{H}_{2}$ is that it is completely soluble in $\mathrm{H}_{2} \mathrm{O}$ and simply transportable that paves it as energy carrier perfectly.

Synthetic photo-synthesis is a photochemical method to renovate sustainable resources into clean fuels and chemicals (for example, $\mathrm{H}_{2} \mathrm{O}_{2}$ ) through sunlight which was expected to solve rising energy requirements. Photocatalytic-based $\mathrm{H}_{2} \mathrm{O}_{2}$ formation is a talented approach to improve energy requirements, as $\mathrm{H}_{2} \mathrm{O}_{2}$ is significant liquid chemical and fuel. However, subsequent dilemma strictly limits the growth of this method:

1. Less selectivity.

2. Less stability (usually $>5$ short-time cycles).

3. Quick charge recombination.

4. Support of hole scavengers.

5. Requirement of $\mathrm{O}_{2}$ saturation.

$\mathrm{TiO}_{2}$ is broadly considered as a photo-catalyst because of its crystal stability, optical, physical and electrical properties as well as biocompatibility $[17,18] . \mathrm{H}_{2} \mathrm{O}_{2}$ manufactured by $\mathrm{TiO}_{2}$ photo-catalytic attracted a great 
concentration [19]. The $\mathrm{TiO}_{2} \mathrm{CB}(-0.19 \mathrm{~V}$ vs. NHE, $\mathrm{pH} 0$ ) bottom is more negative than $2 \mathrm{e}^{-}$reduction of $\mathrm{O}_{2}(0.68 \mathrm{~V})$, which promotes reduction reactions for $\mathrm{H}_{2} \mathrm{O}_{2}$ fabrication, but pristine $\mathrm{TiO}_{2}$ has few disadvantages for example, poor light absorption due to its large BG. Mainly, quantity of $\mathrm{H}_{2} \mathrm{O}_{2}$ generation is much low $(<0.2 \mathrm{mM})$ over pristine $\mathrm{TiO}_{2}$-based photo-catalyst that is away from reasonable level. This may be after the formation of $\mathrm{H}_{2} \mathrm{O}_{2}$; it straight away reacts with surface $\mathrm{Ti}-\mathrm{OH}$ groups and forms $\mathrm{Ti}-\mathrm{OOH}$ complexes. Secondly, Ti-OOH complexes dissociated to Ti-OH and $\mathrm{OH}^{-}$through $\mathrm{e}^{-}$reduction as:

$\mathrm{Ti}-\mathrm{OOH}+\mathrm{H}^{+}+\mathrm{e}^{-} \rightarrow \mathrm{Ti}-\mathrm{OH}+\mathrm{OH}^{-}$.

Numerous surface alteration approaches were applied to boost photo-catalytic fabrication of $\mathrm{H}_{2} \mathrm{O}_{2}$ in $\mathrm{TiO}_{2}$-based photo-catalytic method, for example surface fluorination and surface complexation. The $\mathrm{g}_{-} \mathrm{C}_{3} \mathrm{~N}_{4}$ is analog to graphite, and metal-free polymer n-type SCs have stacked 2D configuration of tri-s-triazine linked through tertiary amines [38]. In consequence of its exceptional structural, electrical, optical, and physicochemical properties, $\mathrm{g}-\mathrm{C}_{3} \mathrm{~N}_{4}$ is familiar as a novel class of multipurpose materials for catalytic, electronic, and energy uses [39]. Wang and coauthors (2009) first revealed photo-catalytic properties of g- $\mathrm{C}_{3} \mathrm{~N}_{4}$ on $\mathrm{H}_{2}$ and $\mathrm{O}_{2}$ evolution [40], and g- $\mathrm{C}_{3} \mathrm{~N}_{4}$-based photo-catalysts have attracted boosting interest worldwide [41]. BG of $\mathrm{g}-\mathrm{C}_{3} \mathrm{~N}_{4}$ is $\sim 2.7 \mathrm{eV}$ which is similar to optical wavelength of $\sim 460 \mathrm{~nm}$, that makes it a possible vis-light-active photo-catalyst. Additionally, $\mathrm{g}-\mathrm{C}_{3} \mathrm{~N}_{4}$ has photo-catalytic capability for $\mathrm{H}_{2} \mathrm{O}$ reduction and oxidation because of its suitable BGs [42]. Hypothetically, g- $\mathrm{C}_{3} \mathrm{~N}_{4}$ is a good photo-catalyst applicant for $\mathrm{H}_{2} \mathrm{O}_{2}$ formation [10] as its $\mathrm{CB}$ position $(-1.3 \mathrm{~V}$ vs. NHE) is correctly located to make possible $\mathrm{O}_{2}$ reduction $(-0.28 \mathrm{~V}$ vs. NHE), while VB potential (1.4 V vs. NHE) is smaller compared to metal oxides that can efficiently avoid oxidative disintegration of $\mathrm{H}_{2} \mathrm{O}_{2}$. As such, $\mathrm{g}_{-} \mathrm{C}_{3} \mathrm{~N}_{4}$ rapidly becomes attractive in the field of photo-catalysis $\mathrm{H}_{2} \mathrm{O}_{2}$ formation [43]. However, photo-catalytic $\mathrm{H}_{2} \mathrm{O}_{2}$ formation activity of $\mathrm{g}-\mathrm{C}_{3} \mathrm{~N}_{4}$ is still limited via low effectiveness in consequence of some adverse parameters, generally including lower surface area, inadequate vis-light harvesting, and quick recombination of photo-induced $\left(\mathrm{e}^{-}-\mathrm{h}^{+}\right)$-pairs. Protocols, for instance engineering structures, controlled defects, loaded noble metal nanoparticles, doping elementals, and heterogenization, were later applied to improve g- $\mathrm{C}_{3} \mathrm{~N}_{4}$-based photo-catalytic $\mathrm{H}_{2} \mathrm{O}_{2}$ formation.

\section{2D/2D Hetero-junctions for Catalysis}

The description of hetero-junction, initially developed from SC-SC (S-S) junction, now has been elaborated to scope metal-SC (M-S) junction and still nontypical hetero-structures of SCs and ionic conductors [136]. Mostly, edge coupling of two components in a hetero-junction could make band arrangement or repairing contact after Fermi levels equilibration (or work functions) at interface following Anderson's rule or Schottky-Mott rule for S-S or M-S junctions, correspondingly. There is an agreement in previous work that re-localization of charge carriers at hetero-junctions edge may make easy catalytic activity of as-fabricated materials or devices.

\subsection{Photo-catalytic $\mathrm{H}_{2}$ Production}

Since 1972, Fujishima and Honda [37] discovered $\mathrm{H}_{2} \mathrm{O}$ splitting at $\mathrm{TiO}_{2}$ electrode under UV light irradiation; at the same time, numerous efforts were dedicated to photocatalytic $\mathrm{H}_{2}$-production [136]. The photo-catalytic is a procedure that produces $\mathrm{H}_{2}$ (and $\mathrm{O}_{2}$ ) using reduced or oxidized adsorbed $\mathrm{H}_{2} \mathrm{O}$ through photo-generated $\mathrm{e}^{-} \mathrm{s}$ and $\mathrm{h}^{+} \mathrm{s}$ at SC catalysts' surface. Due to quick recombination of photogenerated $\left(\mathrm{e}^{-}-\mathrm{h}^{+}\right)$in catalysts, for $\mathrm{H}_{2}$ production effectiveness was still far from prerequisite practical applications. Therefore, 2D/2D layered composite photo-catalysts with suitably formed hetero-junctions are moderately promising for increasing $\mathrm{H}_{2}$-production effectiveness via supporting separation of photo-generated $\left(\mathrm{e}^{-}-\mathrm{h}^{+}\right)$[270]. In comparison with UV light with small percentage of the solar emission, vis-light (almost $50 \%$ of solar radiation) determined that the photo-catalysts are more capable for high proficient sunlight utilization and photo-catalytic activity. Zhang et al. [271] developed a type of "sheet on sheet" hierarchical heterostructure for vis-light-based photo-catalytic $\mathrm{H}_{2}$-production via in situ development of $\mathrm{ZnIn}_{2} \mathrm{~S}_{4}$ 2D-NSs on sheetlike g- $\mathrm{C}_{3} \mathrm{~N}_{4}$ surfaces. g- $\mathrm{C}_{3} \mathrm{~N}_{4}$ was one of the most talented photo-catalysts because of its good stability, non-toxicity, exceptional electronic configuration, and cost efficiency. Its photo-catalytic effectiveness is restricted through poor light harvesting effectiveness and quick recombination of photogenerated charge carriers. Combining vis-active $\mathrm{ZnIn}_{2} \mathrm{~S}_{4}$ 2D-NSs, the above shortcomings were overcome based on 
hetero-junction contact interface that can persuade proficient interfacial transfer of photo-generated $\left(\mathrm{e}^{-}-\mathrm{h}^{+}\right)$from $\mathrm{g}-\mathrm{C}_{3} \mathrm{~N}_{4}$ to $\mathrm{ZnIn}_{2} \mathrm{~S}_{4}$ and delayed charge recombination depending on measurement findings of surface photo-voltage and PL of $\mathrm{ZnIn}_{2} \mathrm{~S}_{4} / \mathrm{g}-\mathrm{C}_{3} \mathrm{~N}_{4}$ hetero-structures. Both suppressed charge recombination on $\mathrm{g}-\mathrm{C}_{3} \mathrm{~N}_{4} \mathrm{NSs}$ and enhanced photo-generated charge carriers in $\mathrm{ZnIn}_{2} \mathrm{~S}_{4} \mathrm{NSs}$ give amazing improvement on photo-catalytic activity of $\mathrm{ZnIn}_{2} \mathrm{~S}_{4} / \mathrm{g}-\mathrm{C}_{3} \mathrm{~N}_{4}$ heterostructures for $\mathrm{H}_{2}$-production.

Enhanced photo-catalytic activity should also be contributed to increase in surface active sites and extension of light absorption via $\mathrm{ZnIn}_{2} \mathrm{~S}_{4}$ NSs combination. The overall photocatalysts-based $\mathrm{H}_{2} \mathrm{O}$ splitting was also developed for production of $\mathrm{O}_{2}$ and $\mathrm{H}_{2}$ concurrently, but it is still a major confront [270]. To fulfill redox potential for overall $\mathrm{H}_{2} \mathrm{O}$ splitting, photo-catalysts $\mathrm{CBM}$ must more negative as compared to $\mathrm{E}_{0}$ of $\mathrm{H}^{+} / \mathrm{H}_{2}\left(0 \mathrm{~V}\right.$ vs. normal $\mathrm{H}_{2}$ electrode (NHE)) and VBM should be more positive compared to oxidation potential of $\mathrm{O}_{2} / \mathrm{H}_{2} \mathrm{O}(1.23 \mathrm{~V})$. Over-potential related to charge transfer and minimum BG of photo-catalysts for efficient $\mathrm{H}_{2} \mathrm{O}$ splitting is always superior compared to theoretical value $(1.23 \mathrm{eV})$. Therefore, Liao et al. formed 2D $\mathrm{MoS}_{2} /$ AlN(GaN)-layered hetero-structures as extremely competent vis-light photo-catalysts for overall $\mathrm{H}_{2} \mathrm{O}$ splitting. The $\mathrm{H}_{2}$ and $\mathrm{O}_{2}$ were formed at opposite surfaces of hetero-structures, because AlN (GaN) and $\mathrm{MoS}_{2}$ SLs act as $\mathrm{e}^{-}$donor and $\mathrm{e}^{-}$acceptor in this hetero-junction photo-catalyst, respectively. Pristine $\mathrm{MoS}_{2}$ using a direct $\mathrm{BG}$ of $1.9 \mathrm{eV}$ was a potential vis-light-driven photo-catalyst and is confirmed not efficient in $\mathrm{H}_{2} \mathrm{O}$ splitting. Group III nitrides (AlN or GaN) SLs with good thermal/chemical stability and highly thermal conductance have demonstrated a good option to manufacture SLs $\mathrm{MoS}_{2}$ hetero-structures, to improve photo-catalytic activity. Moreover, there was only around $2 \%$ lattice mismatch among h-AlN (GaN) and $\mathrm{MoS}_{2}$ SLs, which was major benefit for manufacturing of hetero-structures. The $\mathrm{MoS}_{2} /$ $\mathrm{AlN}$ and $\mathrm{MoS}_{2} / \mathrm{GaN}$ hetero-structures were calculated to be capable photo-catalysts under vis-light irradiation because of proper BGs for $\mathrm{H}_{2} \mathrm{O}$ splitting and good optical absorption [136].

In recent times, polymers were supposed to be one of the potential alternatives in photo-catalytic overall $\mathrm{H}_{2} \mathrm{O}$ splitting (a)

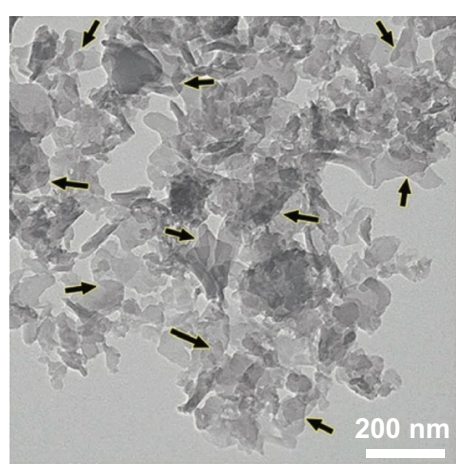

(b)

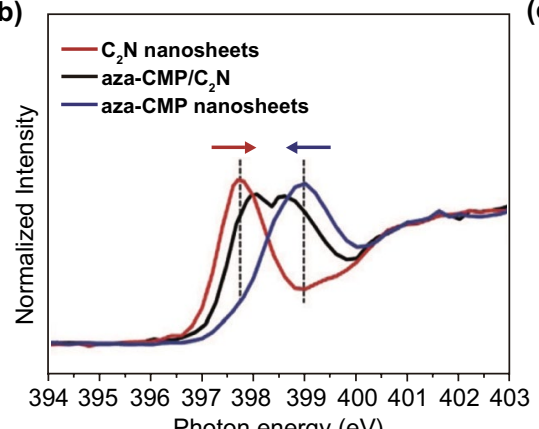

Photon energy $(\mathrm{eV})$

(d) Potential, $\mathrm{pH}=7$

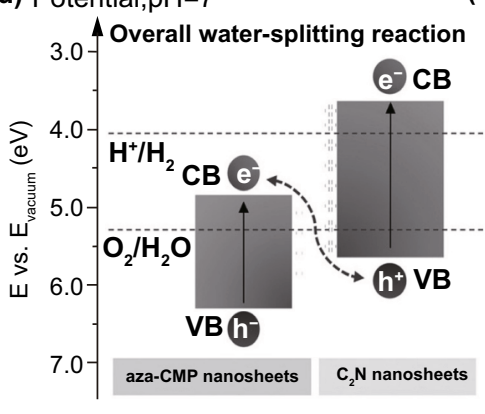

(e)

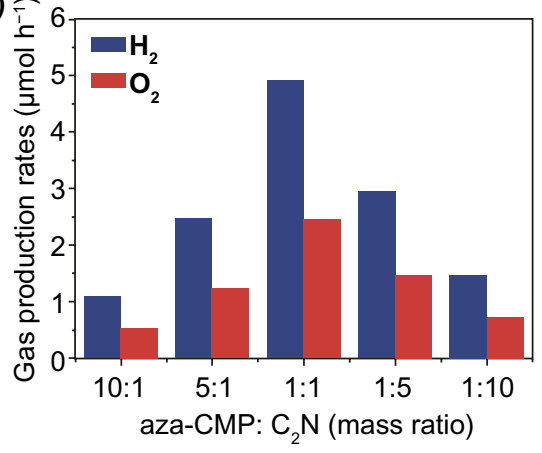

(c)
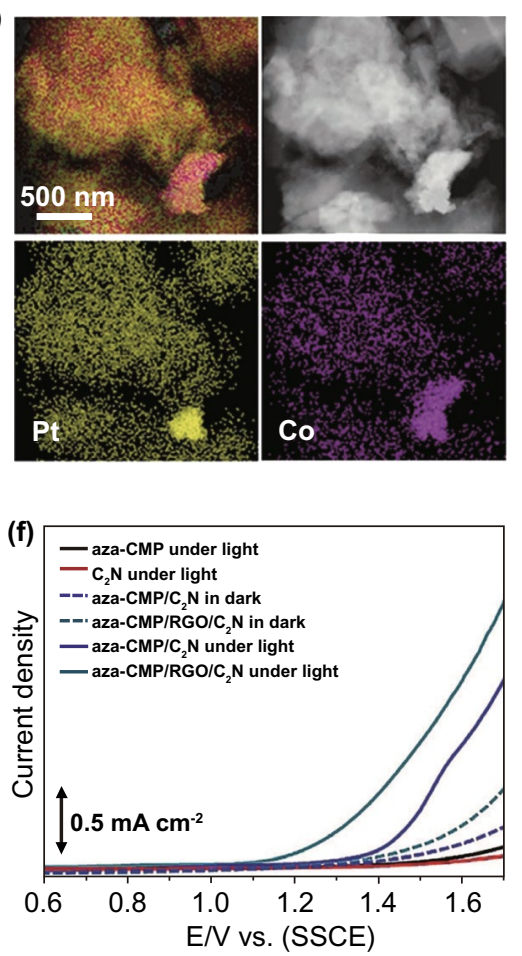

Fig. 24 a TEM image. b NK-edge XANES of aza-CMP, $\mathrm{C}_{2} \mathrm{~N}$, and aza-CMP/C ${ }_{2} \mathrm{~N}$. c HAADF-STEM images and elemental mapping (Pt-labeled $\mathrm{C}_{2} \mathrm{~N}$ and Co-labeled aza-CMP). d Overall $\mathrm{H}_{2} \mathrm{O}$ splitting activity. e Scheme showing aza-CMP/ $\mathrm{C}_{2} \mathrm{~N}$ hetero-structures electronic band structures. $\mathbf{f}$ $\mathrm{J}-\mathrm{V}$ curves of catalysts in dark and under light irradiation. Adapted with permission from Ref. [274] 
because of its engineering molecular structures [272]. To remove limitation of single-element polymer with inadequate $\mathrm{E}_{0}, 2 \mathrm{D} / 2 \mathrm{D}$ polymer hetero-junction photo-catalysts with Z-scheme configuration were designed from imitating two-step excitation way of plants, where light-driven two half reactions of $\mathrm{H}_{2} \mathrm{O}$ splitting and glucose formation are divided spatially [273]. The Z-scheme-based polymer derived from 2DMs was proved to be an effective pathway to get appropriate energy levels for enough reaction kinetics and allow proficient charge transfer in overall $\mathrm{H}_{2} \mathrm{O}$ splitting.
Wang et al. [270] synthesized hetero-structures (aza-CMP/ $\mathrm{C}_{2} \mathrm{~N}$ ) consisting of aza-conjugated microporous polymers (CMP) and $\mathrm{C}_{2} \mathrm{~N}$ NSs as $2 \mathrm{D}$ polymer-related Z-scheme systems for competent photo-catalytic overall $\mathrm{H}_{2} \mathrm{O}$ splitting. The aza-CMP/C ${ }_{2} \mathrm{~N}$ hetero-structures were synthesized via mixing and consequently annealing of $\mathrm{CMP}$ and $\mathrm{C}_{2} \mathrm{~N}$ NSs. The as-fabricated stacked NSs of aza-CMP/C ${ }_{2} \mathrm{~N}$ with abundant overlapped areas might be exposed via the TEM analysis in Fig. 24a. The XANES spectroscopy, HAADFSTEM image, and elemental mapping were utilized to show (a)

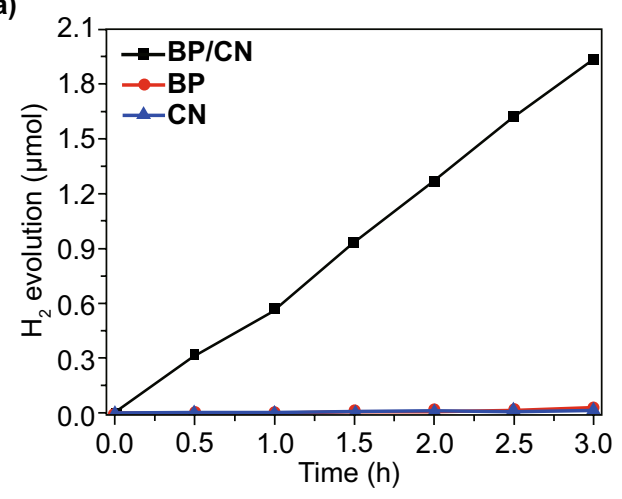

(c)

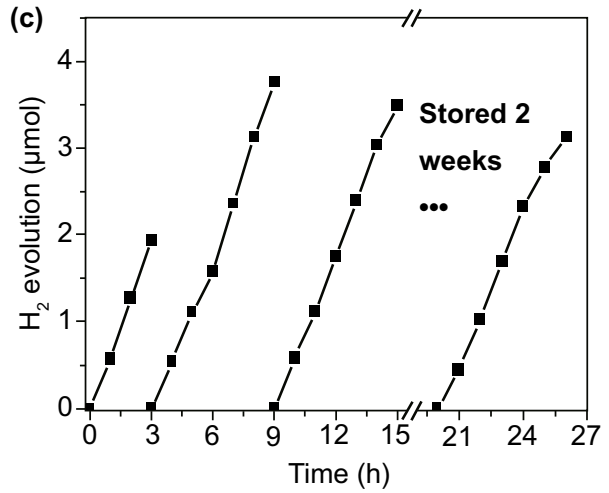

(e)

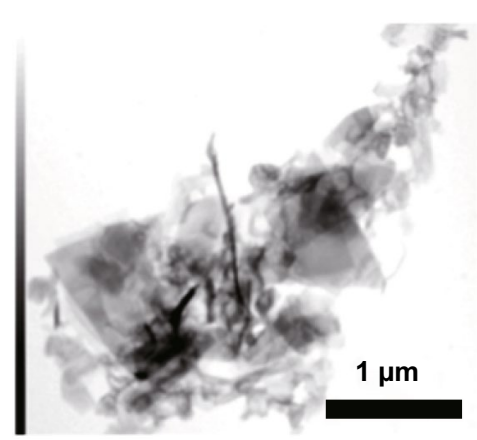

(b)
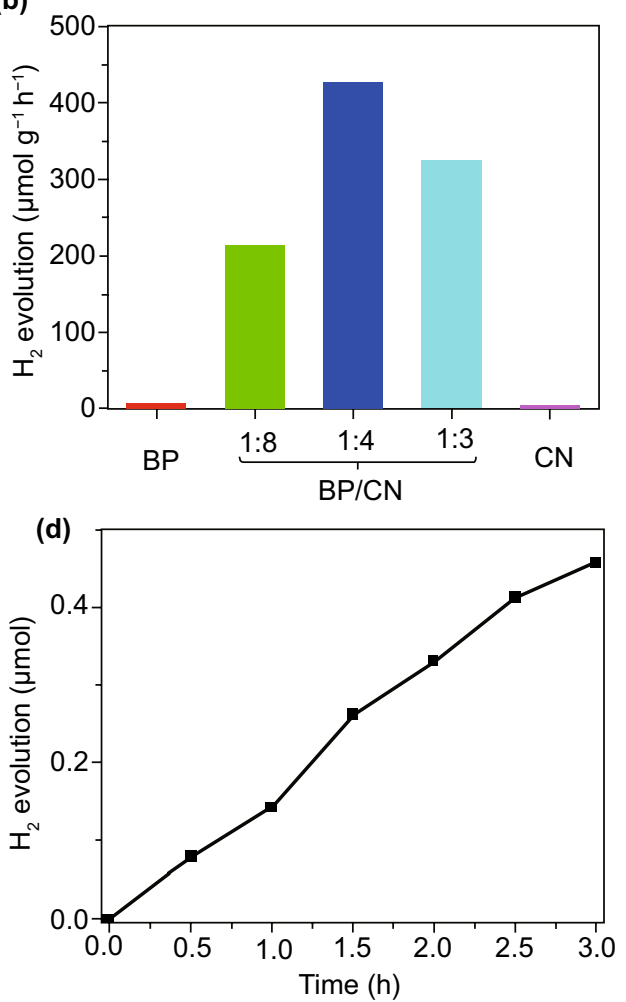

(f)

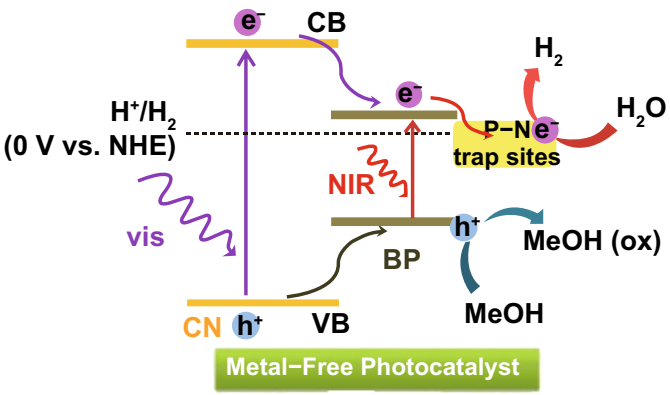

Fig. 25 a Photo-catalytic $\mathrm{H}_{2}$ evolution based on different catalysts with $>420 \mathrm{~nm}$. b Effect of BP/CN ratio in BP/CN on photo-catalytic $\mathrm{H}_{2}$ production rate under vis-light irradiation for $3 \mathrm{~h}$. c Cycle stability test on BP/CN photo-catalytic $\mathrm{H}_{2}$ production under vis-light irradiation. d Photocatalytic $\mathrm{H}_{2}$ production based on BP/CN with>780 nm light irradiation. e HAADF-STEM image. f Scheme for photo-catalytic $\mathrm{H}_{2}$ evolution utilizing BP/CN. Adapted with permission from Ref. [275] 
interlayer relations as well as homogeneous combination of aza-CMP and $\mathrm{C}_{2} \mathrm{~N}$ NSs (Fig. 24b).

Investigation on photo-catalytic activity showed that $\mathrm{H}_{2}$ and $\mathrm{O}_{2}$ (molar ratio 2:1) were concurrently formed from pristine $\mathrm{H}_{2} \mathrm{O}$ with aza-CMP/C ${ }_{2} \mathrm{~N}$ hetero-structures as photocatalysts under vis-light (>420 nm) irradiation, whereas both aza-CMP and $\mathrm{C}_{2} \mathrm{~N}$ components are inactive. In addition, aza-CMP/ $\mathrm{C}_{2} \mathrm{~N}$ hetero-structures with 1:1 mass ratio showed optimum photo-catalytic performance $\left(\mathrm{H}_{2}\right.$ evolution rate: $5.0 \mathrm{mmol} \mathrm{h}^{-1}$, solar to $\mathrm{H}_{2}$ conversion efficiency: $0.23 \%$, evident QE at $600 \mathrm{~nm}$ : 4.3\%) (Fig. 24c). Figure 24d presents the energy band position of aza-CMP/C $\mathrm{C}_{2} \mathrm{~N}$ hetero-structures, which indicates that photo-generated $\mathrm{e}^{-} \mathrm{s}$ in $\mathrm{C}>\mathrm{B}$ of aza$\mathrm{CMP}$ were quickly recombined with photo-generated $\mathrm{h}^{+} \mathrm{s}$ at $\mathrm{VB}$, of $\mathrm{C}_{2} \mathrm{~N}$ at their interface, while other photo-generated $\mathrm{e}^{-} \mathrm{s}$ and $\mathrm{h}^{+} \mathrm{s}$ participated in $\mathrm{H}_{2} \mathrm{O}$ redox reaction. Credit to as-constructed hetero-structures, both charge separation and transfer were assisted in aza-CMP/C ${ }_{2} \mathrm{~N}$ composites (confirmed via outcomes of electrochemical impedance measurements and transient photo-current) and therefore increased photo-catalytic performance.

As a move toward green chemistry, sequence of metalfree photo-catalysts, e.g., g- $\mathrm{C}_{3} \mathrm{~N}_{4}, \mathrm{BP}$, boron nitride ( $\left.\mathrm{BN}\right)$, and boron carbide (BC), was highly preferred to be studied

Table $1 \mathrm{H}_{2}$-production based on photo-catalysts

\begin{tabular}{|c|c|c|c|c|}
\hline Materials & Co-catalyst & Light source & $\begin{array}{l}\mathrm{H}_{2} \text { yield } \\
\left(\mu \mathrm{mol} \mathrm{h}{ }^{-1} \mathrm{~g}^{-1}\right)\end{array}$ & References \\
\hline $\mathrm{CdS}$ & $0.5 \mathrm{wt} \% \mathrm{Pd}, 4 \mathrm{wt} \% \mathrm{MoS}_{2}, 5 \mathrm{wt} \%$ polyaniline & Daylight fluorescent lamp & 570 & {$[278]$} \\
\hline $\mathrm{CdSe}$ & $\mathrm{Pt}$ & $\lambda=300 \mathrm{~W}$ & 1.65 & [279] \\
\hline UiO-66/CdS & $1.5 \mathrm{wt} \% \mathrm{MoS}_{2}$ & $\lambda \geq 400 \mathrm{~nm}$ & 12,426 & [280] \\
\hline $\mathrm{In}_{2} \mathrm{~S}_{3} / \mathrm{CdS}$ & $0.2 \mathrm{wt} \% \mathrm{MoS}_{2}$ & $\lambda \geq 420 \mathrm{~nm}$ & 625.8 & [281] \\
\hline $\mathrm{CdS}$ & 0.4 wt $\%$ rGO, 2 wt $\% \mathrm{MoS}_{2}$ & $500 \mathrm{~W}$ UV-Vis lamp & 6857 & [282] \\
\hline $\mathrm{CdS}$ & $1.33 \mathrm{wt} \%$ graphene, $0.67 \mathrm{wt} \% \mathrm{MoS}_{2}$ & $\lambda \geq 420 \mathrm{~nm}$ & 9000 & [283] \\
\hline $\mathrm{CdS}$ & $2 \mathrm{wt} \%$ single-layer (SL) $\mathrm{MoS}_{2}$ & $\lambda \geq 420 \mathrm{~nm}$ & 10,050 & [284] \\
\hline $\mathrm{CdS}$ & $0.2 \mathrm{wt} \% \mathrm{MoS}_{2}$ & $\lambda \geq 420 \mathrm{~nm}$ & 5330 & {$[285]$} \\
\hline $\mathrm{CdS}$ & $0.2 \mathrm{wt} \% \mathrm{MoS}_{2}$ & $\lambda \geq 420 \mathrm{~nm}$ & $\sim 5400$ & [286] \\
\hline CdS NSs & $1 \% \mathrm{MoS}_{2} \mathrm{NSs}$ & $\lambda \geq 400 \mathrm{~nm}$ & 8720 & [287] \\
\hline g- $\mathrm{C}_{3} \mathrm{~N}_{4}$ & - & $\lambda>420 \mathrm{~nm}$ & 3.2 & [288] \\
\hline $\mathrm{g}-\mathrm{C}_{3} \mathrm{~N}_{4}$ & $\mathrm{Pt}$ & $\lambda>420 \mathrm{~nm}$ & 106.9 & [288] \\
\hline $\mathrm{g}-\mathrm{C}_{3} \mathrm{~N}_{4}$ & $\mathrm{Pt}$ & $\lambda>300 \mathrm{~nm}$ & 23,468 & {$[288]$} \\
\hline Graphene & $\mathrm{CdS}$ & $300 \mathrm{~W}$ Xe & 1050 & [289] \\
\hline $1 \mathrm{H}-\mathrm{MoS}_{2}$ & - & $100 \mathrm{~W}$ halogen & 50 & [290] \\
\hline $\mathrm{NrGO}-\mathrm{MoS}_{2}$ & - & 100/400 W halogen & $10.8 / 42 \mathrm{k}$ & [290] \\
\hline $1 \mathrm{~T}-\mathrm{MoS}_{2}$ & - & $100 \mathrm{~W}$ halogen & 26,000 & [290] \\
\hline $1 \mathrm{~T}-\mathrm{MoSe}_{2}$ & - & $100 \mathrm{~W}$ halogen & 62,000 & [291] \\
\hline $1 \mathrm{~T}-\mathrm{WS}_{2}$ & $\mathrm{TiO}_{2}$ & $300 \mathrm{~W}$ Xe & 2570 & [292] \\
\hline $2 \mathrm{H}-\mathrm{WS}_{2}$ & $\mathrm{TiO}_{2}$ & $300 \mathrm{~W}$ Xe & 225 & [292] \\
\hline $\mathrm{MoS}_{2}$ & $\mathrm{CdS}$ & $\lambda>420 \mathrm{~nm}$ & 1472 & [293] \\
\hline $\mathrm{WS}_{2}$ & $\mathrm{CdS}$ & $\lambda>420 \mathrm{~nm}$ & 1984 & {$[24]$} \\
\hline $\mathrm{SnS}_{2}$ & - & $300 \mathrm{~W}$ Xe & 1060 & [104] \\
\hline $\mathrm{ZnIn}_{2} \mathrm{~S}_{4}$ & - & $300 \mathrm{~W}$ Xe & 57 & [294] \\
\hline $\mathrm{ZnIn}_{2} \mathrm{~S}_{4}$ & $\mathrm{Pt}$ & $300 \mathrm{~W}$ Xe & 213 & [294] \\
\hline $\mathrm{Zn}-\mathrm{In}-\mathrm{S}$ & $\mathrm{Pt}$ & $400 \mathrm{~W} \mathrm{Hg}$ & 229 & [295] \\
\hline $\mathrm{Zn}-\mathrm{In}-\mathrm{S}$ & $\mathrm{Pt}+\mathrm{NaCl}$ & $400 \mathrm{~W} \mathrm{Hg}$ & 1056 & [295] \\
\hline $\mathrm{TiO}_{2}$ & $\mathrm{Pt}$ & $350 \mathrm{~W}$ Xe & 1667.5 & [296] \\
\hline $\mathrm{CdSe}$ & $2 \% \mathrm{CoP}$ & $350 \mathrm{~W}$ Xe & 56.3 & [277] \\
\hline $\mathrm{CdS}$ & $\mathrm{WS}_{2}$ & $300 \mathrm{~W}$ Xe lamp & 14.1 & [297] \\
\hline
\end{tabular}


because of their profusion, inexpensive, and excellent stability. The study of a competent and stable metal-free photo-catalyst with broad spectrum solar absorption for photo-catalytic $\mathrm{H}_{2}$-production remains a major issue. Zhu et al. [275] productively formed a 2D layered hetero-structures consisting of $\mathrm{BP}$ and graphitic carbon nitride $(\mathrm{CN})$ (BP/CN) as metal-free photo-catalysts in $\mathrm{H}_{2}$-evolution for vis to near-IR region for the very first time. Compared to a single element (BP or $\mathrm{CN}$ ), $\mathrm{BP} / \mathrm{CN}$ hetero-structures showed considerably enhanced photo-catalytic activity, and $\mathrm{H}_{2}$-evolution for $3 \mathrm{~h}$ obtained $1.93 \mu \mathrm{mol}, 0.46 \mu \mathrm{mol}$ under $>420 \mathrm{~nm}>780 \mathrm{~nm}$ light irradiation, respectively (Fig. 25a, d). It is mostly due to proficient interface charge transfer based on strong interface interaction in $\mathrm{CN}$ and $\mathrm{BP}$ that restrained recombination and improved the partition of photo-generated $\left(\mathrm{e}^{-}-\mathrm{h}^{+}\right)$-pairs (Fig. 25f). After excitation in vis-light, a charge migration from $\mathrm{CN}$ to combine BP stimulated through hetero-junction interfacial effect in $\mathrm{BP} / \mathrm{CN}$ can be established through consequences of timeresolved diffuse reflectance spectroscopy. In NIR excitation case, just BP was excited and extensive excitation duration is obtained from competent electron entrapping via P-N coordinate bond at hetero-junction edge [136].

Jingrun Ran et al. [276] studied metal-free photo-catalysts of metal-free $2 \mathrm{D} / 2 \mathrm{D}$ vdWs phosphorene/g- $\mathrm{C}_{3} \mathrm{~N}_{4}$ hetero-structure. The synthesized nano-composite showed improved vis-light photo-catalytic $\mathrm{H}_{2}$ production activity of $\sim 571 \mu \mathrm{mol} \mathrm{h} \mathrm{h}^{-1} \mathrm{~g}^{-1}$ in $18 \mathrm{v} \%$ lactic acid aqueous solution. This enhanced activity occurs due to intimate electronic coupling at $2 \mathrm{D} / 2 \mathrm{D}$ interface, which introduced a new metal-free phosphorene/g- $\mathrm{C}_{3} \mathrm{~N}_{4}$ photo-catalyst and formed 2D/2D vdWs hetero-junction for uses in catalysis, electronics, and optoelectronics. Qixiao Gai et al. [277] synthesized 2D CdS and 2D CoP NSs, oxidation and phosphidation process. Then, 2D-2D CdSe/CoP photo-catalysts were formed by ultrasonically dispersing the mixed solution of $\mathrm{CdS}$ and CoP. The $\mathrm{CdSe} / \mathrm{CoP}$ with $2 \% \mathrm{CoP}$ loading amounts showed a maximum photo-catalytic performance of $56.3 \mathrm{mmol} \mathrm{g}^{-1} \mathrm{~h}^{-1}$ under vis-light irradiation that is 11.3 times as high as bare CdS. The improved photo-catalytic activity of $\mathrm{CdS}$ eCoP should be due to the following two points: (1) high catalytic activity of CoP; (2) highly proficient separation and transfer of $\left(\mathrm{e}^{-}-\mathrm{h}^{+}\right)$-pairs photo-generated in CdS because of synthesized 2D-2D hetero-structure. In Table $1, \mathrm{H}_{2}$-production-based photo-catalysts are summarized.

\subsection{Photo-catalytic Pollutant Degradation}

In industrial progress, mass discharge of poisonous wastes (e.g., agrochemicals, dyes, and antibiotics) [136] has become worldwide a rigorous hazard to $\mathrm{H}_{2} \mathrm{O}$ resources and human physical condition [298]. Besides civilizing environment policies and system, development of an eco-friendly solution for eradicating pollution is dreadfully in demand. Among possible solutions, photo-catalytic decomposition of organic contaminants via in situ very reactive species is supposed to be green, cost-efficient, and talented move to deal with pollution matters. Even though numerous types of single ingredient photo-catalysts were developed, mostly suffer from a poor photo-catalytic activity and are not sufficiently efficient for real applications. A series of 2D/2D layer hetero-structures was generated for organic photo-degradation, showing potential uses. The $2 \mathrm{D} / 2 \mathrm{D}$ hetero-structures consisting of $\mathrm{AgIO}_{3}$ and g- $\mathrm{C}_{3} \mathrm{~N}_{4} \mathrm{NSs}\left(\mathrm{AgIO}_{3} / \mathrm{g}-\mathrm{C}_{3} \mathrm{~N}_{4}-\mathrm{NS}\right)$ were successfully formed for photo-catalytic waste $\mathrm{H}_{2} \mathrm{O}$ treatment after vis-light exposure by $\mathrm{Li}$ et al. [299]. The ultra-thin $\mathrm{g}_{-} \mathrm{C}_{3} \mathrm{~N}_{4} \mathrm{NSs}$ as polymeric organic SCs material were exhibiting superior vis-light response. Photo-catalytic performance of $\mathrm{AgIO}_{3} / \mathrm{g}-\mathrm{C}_{3} \mathrm{~N}_{4} \mathrm{NSs}$ hetero-structures was considerably higher than that of single $\mathrm{AgIO}_{3}$ or $\mathrm{g}_{-} \mathrm{C}_{3} \mathrm{~N}_{4} \mathrm{NSs}$ for organic dyes degradation. Noticeably, degradation reaction rate constant of rhodamine $\mathrm{B}(\mathrm{RhB})$ than as-synthesized $\mathrm{AgIO}_{3} / \mathrm{g}-\mathrm{C}_{3} \mathrm{~N}_{4}$-NS sample was approximately 22.86 times higher as compared to hetero-structures composed of $\mathrm{AgIO}_{3}$ and bulk g- $\mathrm{C}_{3} \mathrm{~N}_{4}\left(\mathrm{AgIO}_{3} / \mathrm{g}-\mathrm{C}_{3} \mathrm{~N}_{4}-\mathrm{B}\right)$. It shows significance of 2DMs for construction of hetero-junction photo-catalysts (Fig. 26b).

A possible photo-catalytic method was also proposed in this work: The photo-induced $\mathrm{e}^{-} \mathrm{s}$ on $\mathrm{CB}$ of $\mathrm{g}-\mathrm{C}_{3} \mathrm{~N}_{4}-\mathrm{NS}$ can move to $\mathrm{AgIO}_{3} \mathrm{CB}$ under vis-light irradiation that promoted photo-generated $\left(\mathrm{e}^{-}-\mathrm{h}^{+}\right)$-pairs separation in $\mathrm{g}-\mathrm{C}_{3} \mathrm{~N}_{4}-\mathrm{NS}$ (Fig. 26c). Considerably decreased steady-state PL spectra peak intensity and enhanced lifetime of charge carriers in PL spectra of $\mathrm{AgIO}_{3} / \mathrm{g}-\mathrm{C}_{3} \mathrm{~N}_{4} \mathrm{NSs}$ as compared with that of single $\mathrm{C}_{3} \mathrm{~N}_{4} \mathrm{NSs}$ indicated superior $\mathrm{e}^{-}$-migration and charge separation effectiveness at hetero-junction edge. To consider $\mathrm{O}_{2} / \cdot \mathrm{O}^{2-}$ reduction potential, the oxidative breakdown of the dye should be due to $\mathrm{h}^{+} \mathrm{s}$ in VB of the $\mathrm{g}-\mathrm{C}_{3} \mathrm{~N}_{4} \mathrm{NSs}$ and - $\mathrm{O}^{2-}$ produced through $\mathrm{e}^{-} \mathrm{s}$ reduction of $\mathrm{O}_{2}$ from $\mathrm{CB}$ of g- $\mathrm{C}_{3} \mathrm{~N}_{4}$ NSs. In another work, diverse quantity of $\mathrm{BiOCl}$ nano-plates is utilized to combine with $\mathrm{C}_{3} \mathrm{~N}_{4} \mathrm{NSs}$ via an easy calcination method. The face-to-face interaction edge 
(a)
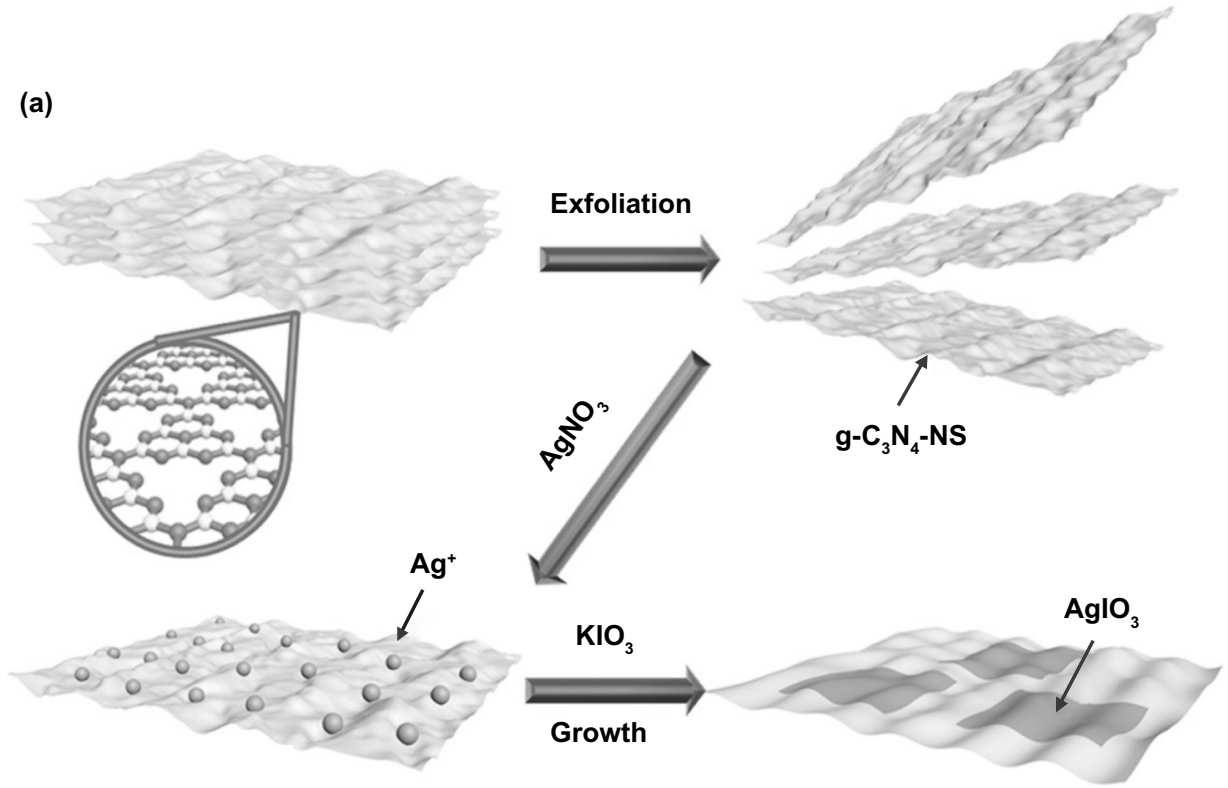

(b)
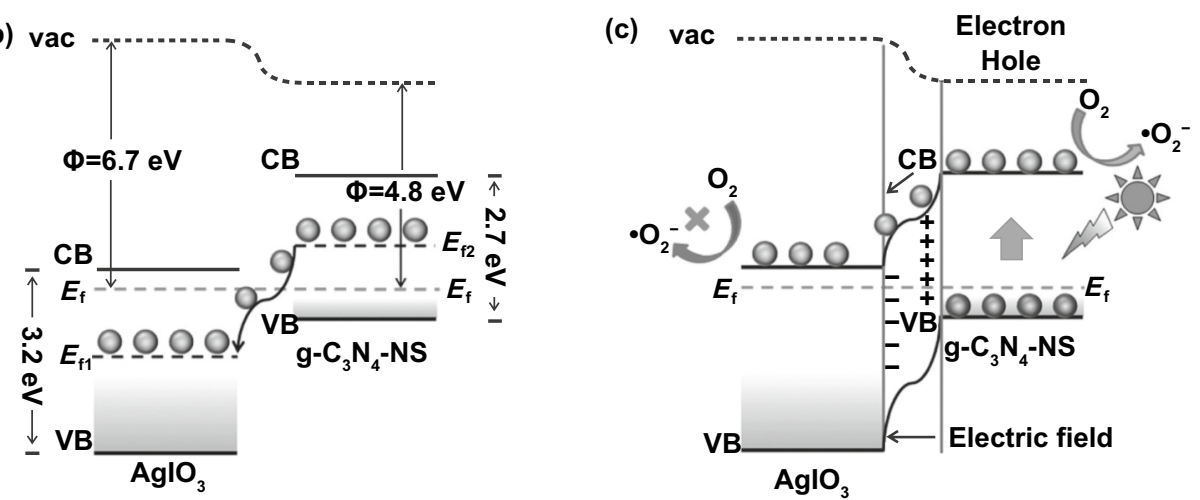

Fig. 26 a Scheme showing $\mathrm{AgIO}_{3} / g-\mathrm{C}_{3} \mathrm{~N}_{4}-\mathrm{NSs}$ composite synthesis. b Photo-catalytic degradation kinetics of RhB after vis-light irradiation. $\mathbf{c}$ Scheme showing charge separation at $\mathrm{AgIO}_{3} / \mathrm{g}-\mathrm{C}_{3} \mathrm{~N}_{4}-\mathrm{NS}$ interface after vis-light irradiation. Adapted with permission from Ref. [299]

of as-synthesized $\mathrm{BiOCl} / \mathrm{C}_{3} \mathrm{~N}_{4}$ hetero-structures was studied, and the significance of contact area of two components was discussed. The superior photo-current intensity and weakened PL intensity of $\mathrm{BiOCl} / \mathrm{C}_{3} \mathrm{~N}_{4}$ hetero-structures as compared with those of $\mathrm{C}_{3} \mathrm{~N}_{4} \mathrm{NSs}$ illustrated separation and migration of photo-generated e-s at interface. The $\mathrm{BiOCl} /$ $\mathrm{C}_{3} \mathrm{~N}_{4}$ hetero-junction photo-catalysts were loaded with $70 \%$ $\mathrm{BiOCl}$ which showed the highest $\mathrm{MO}$ photo-degradation activity in vis-light irradiation. Wang et al. [300] prepared

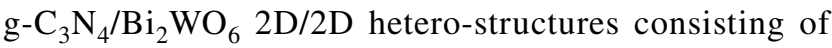
g- $\mathrm{C}_{3} \mathrm{~N}_{4} \mathrm{NSs}$ and $\mathrm{SL} \mathrm{Bi}_{2} \mathrm{WO}_{6} \mathrm{NSs}$ for degradation of ibuprofen under vis-light irradiation. It shows that highly active photo-degradation system might be developed via navigation of charge division, transportation, and consumption at atomic level. Bera et al. [301] prepared a chain of heterostructures consisting of $\mathrm{rGO}$ and $\mathrm{CdS}$ with diverse dimensionality ( $\mathrm{rGO} / \mathrm{CdS}$ ) for photo-catalytic degradation of methylene blue (MB).

The 2D-G as an example of 2D-layered material, whose atomic thickness has outstanding charge transfer ability, can offer conducting $\mathrm{e}^{-}$-channels for separation of the photogenerated charges in the hetero-junction photo-catalyst composed of BG and SC. Through utilizing terephthalic acid (TA) as an example, the $\mathrm{OH}^{\bullet}$ radicals were established to be generated active species for the photo-catalytic decomposition. Therefore, improved $\mathrm{rGO} / \mathrm{CdS}$ activity in comparison with the single $\mathrm{CdS}$ might be explained as follows: Under vis-light irradiation, photo-generated $\mathrm{e}^{-}$in $\mathrm{CB}$ of $\mathrm{CdS}$ is 

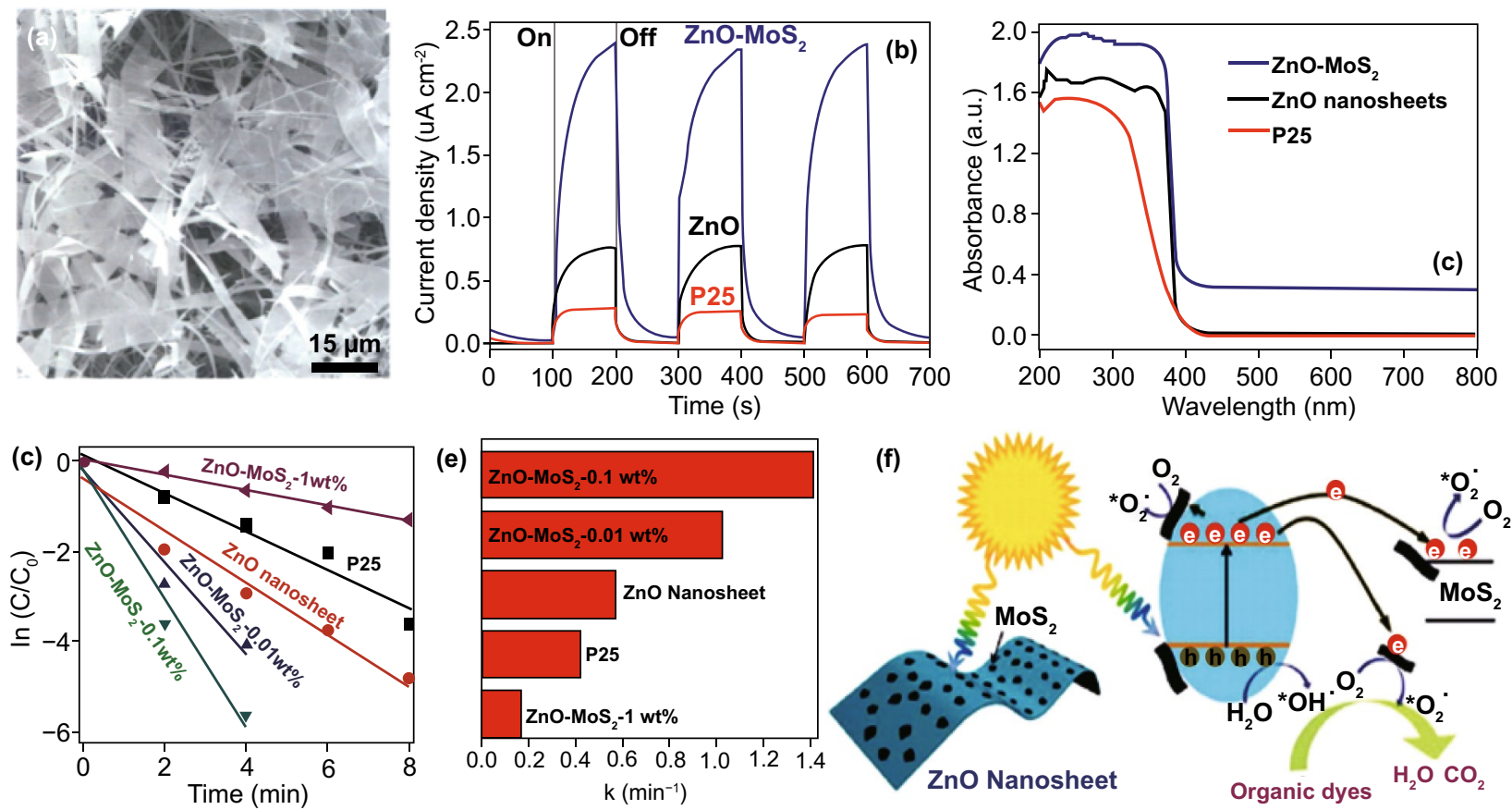

Fig. 27 a $\ln \left(C / C_{0}\right)$ versus time curves of $\mathrm{MB}$ with different photo-catalysts: $\mathrm{ZnO} / \mathrm{MoS}_{2}$ hetero-structures with diverse doping amounts of $\mathrm{MoS}_{2}$ (mass ratio is $0,0.01,0.1$, and $1 \mathrm{wt} \%$ ) and commercially available P25. b Obvious rate constants of MB photo-degradation with different photocatalysts. c Scheme showing photo-catalytic system of $\mathrm{ZnO} / \mathrm{MoS}_{2}$ hetero-structures. Adapted with permission from Ref. [302]

transferred to rGO surface and reacts with adsorbed $\mathrm{O} 2$ that consequently produces $\cdot \mathrm{O}^{2-}$ and $\mathrm{OH}$. Oxidization of both $\mathrm{OH}^{\bullet}$ and photo-generated $\mathrm{h}^{+} \mathrm{s}$ leads to photo-catalytic decomposition of MB dye molecules. Charge migration from $\mathrm{CdS}$ to $\mathrm{rGO}$ takes place at $\mathrm{rGO} / \mathrm{CdS}$ hetero-structures interface, as proved from considerably quenched PL of $\mathrm{CdS}$ components, promoting separation of photo-generated charges and therefore enhancing photo-catalytic activity [136]. In a few cases, the photo-catalytic activity of pollutant degradation might be increased through synergetic effect in hetero-junctions and other configuration tuning.

Yangyang Liu et al. [302] studied a 2D/2D nano-composite photo-catalyst $\left(\mathrm{ZnO} / \mathrm{MoS}_{2}\right)$ derived from P-doped $\mathrm{ZnO}$ NSs with large SSA and $2 \mathrm{D}-\mathrm{MoS}_{2}$ for competent photo-degradation of organic dyes (Fig. 27a-f). The $\mathrm{ZnO} / \mathrm{MoS}_{2}$ hetero-structures with different $\mathrm{MoS}_{2}$ loading amounts (mass ratio is $0,0.01,0.1$, and $1 \mathrm{wt} \%$ ) and commercial P25 are utilized as photo-catalysts for photodegradation calculations that took place in similar experimental conditions (Fig. 27d, e). Comparative findings illustrate that $\mathrm{ZnO} / \mathrm{MoS}_{2}$ with a small loading amount of $\mathrm{MoS}_{2}(0.1 \mathrm{wt} \%)$ would considerably improve photo-catalytic activity in comparison with pristine $\mathrm{ZnO}$ NSs. When $\mathrm{MoS}_{2}$ loading amount was increased from 0.01 to $0.1 \mathrm{wt} \%$, photo-catalytic performance of $\mathrm{ZnO} / \mathrm{MoS}_{2}$ was further improved, but reduced after loading amount of $\mathrm{MoS}_{2}$ and further enhanced to $1 \mathrm{wt} \%$. A huge $\mathrm{MoS}_{2}$-doped concentration would block sunlight that was utilized to force photodegradation of MB and therefore decreases the photocatalytic performance. A photo-catalytic mechanism of $\mathrm{ZnO} / \mathrm{MoS}_{2}$ hetero-structures is proposed in Fig. 27f [136]. Derived from interfacial effect between $\mathrm{ZnO}$ and $\mathrm{MoS}_{2}$, photo-generated $\mathrm{e}^{+} \mathrm{s}$ would migrate from $\mathrm{CB}$ of $\mathrm{ZnO}$ to that of $\mathrm{MoS}_{2}$ that considerably improved the separation of carriers and therefore increase catalytic activity. Increased transport and separation of photo-generated $\mathrm{h}^{+} \mathrm{s}$ and $\mathrm{e}^{-} \mathrm{s}$ stimulated through interfacial effect can be established through increased photo-current density of $\mathrm{MoS}_{2} / \mathrm{ZnO}$ hetero-structures after introduction of $\mathrm{MoS}_{2}$ components. The photo-generated charges react with $\mathrm{O}_{2}$ and $\mathrm{H}_{2} \mathrm{O}$ after 
(a)
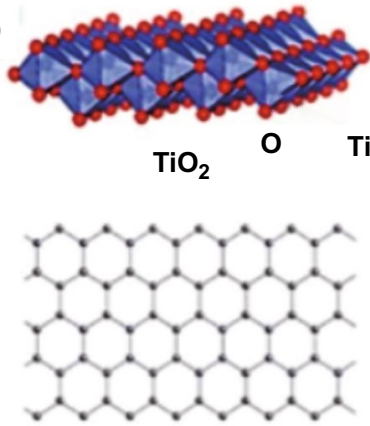

graphene
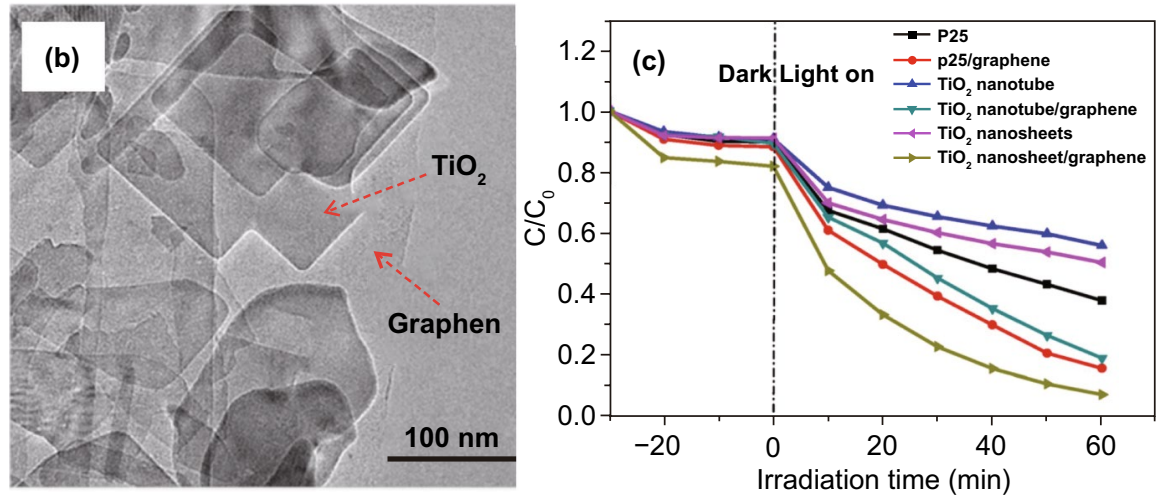

(d)

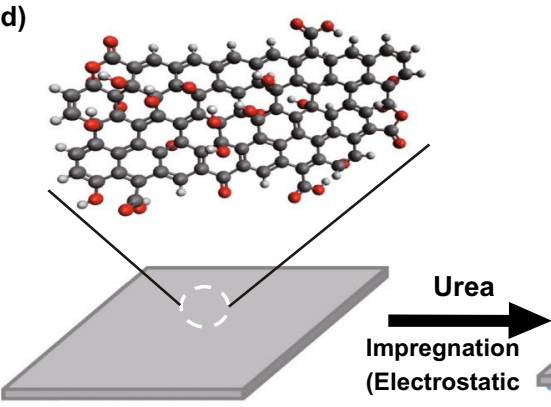

Graphene oxide

attraction)

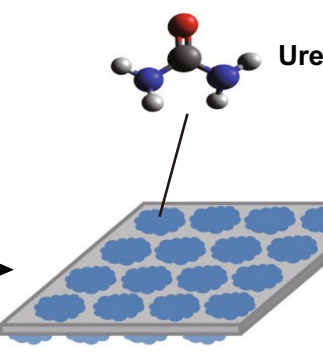

Graphene oxide/urea Urea

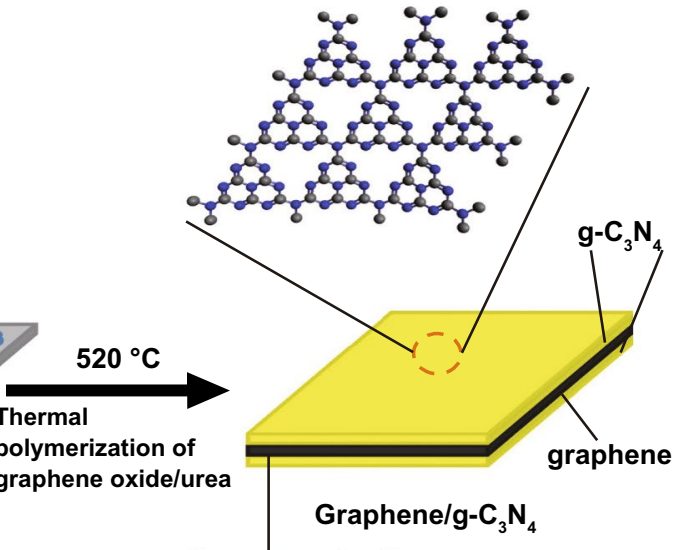

- Carbon atom Oxygen atom

Nitrogen atom Hydrogen atom
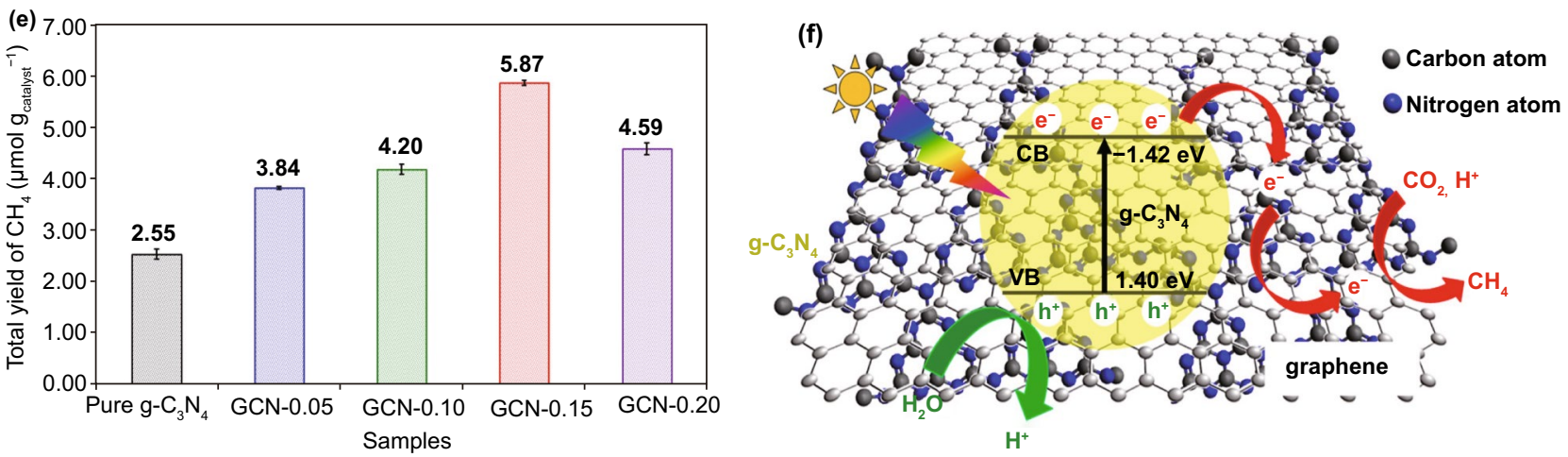

Fig. 28 a $\mathrm{TiO}_{2}$ and graphene NS structures. b TEM and element mapping of graphene/ $/ \mathrm{TiO}_{2}$ hetero-structure. c Photo-catalytic degradation of rhodamine B (RhB) over different photo-catalysts under irradiation of UV light. Adapted with permission from Ref. [304]. d Preparation of GCN samples through one-pot impregnation-thermal reduction approach. e Total $\mathrm{CH}_{4}$ yield over as-synthesized photo-catalysts. f Photo-generated charge transfer in $\mathrm{GCN}$ system for $\mathrm{CO}_{2}$ reduction with $\mathrm{H}_{2} \mathrm{O}$ to make $\mathrm{CH}_{4}$. Adapted with permission from Ref. [303]

relocated to catalyst surface and generated much reactive radicals ( $\mathrm{OH}$ and superoxide anion radicals) to degrade dye molecules. In addition to hetero-junction effect, P loading stimulated defects in ZnO NSs also support photo-catalytic activity as they can enhance light absorption via introduction of energy level between BGs [136]. 


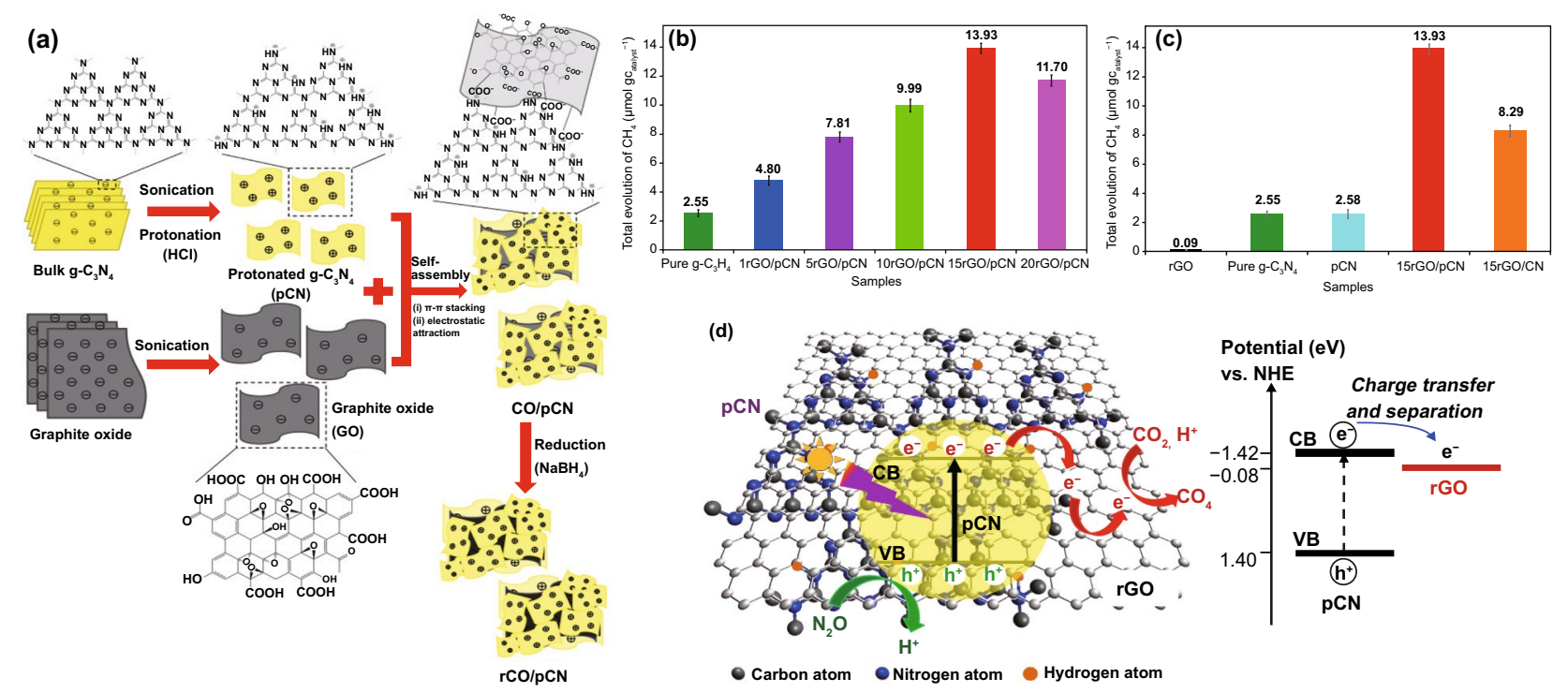

Fig. 29 a Production method of rGO/pCN. b Total production of $\mathrm{CH}_{4}$ over pure $\mathrm{g}_{-} \mathrm{C}_{3} \mathrm{~N}_{4}$ and a series of rGO/pCN photo-catalysts with different rGO contents under vis-light irradiation for $10 \mathrm{~h}$. c Total evolution of $\mathrm{CH}_{4}$ after $10 \mathrm{~h}$ over rGO, pure g- $\mathrm{C}_{3} \mathrm{~N}_{4}, \mathrm{pCN}$, and $15 \mathrm{rGO} / \mathrm{CN}$. d Charge transfer and separation process happening in $\mathrm{rGO} / \mathrm{pCN}$ nano-composite for $\mathrm{CO}_{2}$ reduction to $\mathrm{CH}_{4}$ in the occurrence of $\mathrm{H}_{2} \mathrm{O}$ under vis-light lighting. Adapted with permission from Ref. [305]

\subsection{Photo-catalytic $\mathrm{CO}_{2}$ Reduction}

The 2D materials aptitudes to boost the specific surface area to give elevated surface reactive sites make them a top precedence for photo-catalyst supports. Provided large contact area of 2D/2D composite, contact in graphene and photo-catalyst is anticipated to be enhanced that speeds up transfer and separation of photo-generated $\left(\mathrm{e}^{-}-\mathrm{h}^{+}\right)$-pairs, therefore enhancing their photo-catalytic $\mathrm{CO}_{2}$ reduction activity. According to the above-discussed phenomena, J. Sun et al. [257] fabricated three classes of 2D/0D, 2D/1D, and $2 \mathrm{D} / 2 \mathrm{D}$ graphene/ $\mathrm{TiO}_{2}$ hetero-structures through solvothermal method. So, the $2 \mathrm{D} / 2 \mathrm{D}$ graphene/ $/ \mathrm{TiO}_{2}$ heterostructure showed maximum photo-catalytic effectiveness in contrast to $0 \mathrm{D} / 2 \mathrm{D}$ and $1 \mathrm{D} / 2 \mathrm{D}$ graphene/ $/ \mathrm{TiO}_{2}$ hetero-structures and pristine $\mathrm{TiO}_{2} \mathrm{NSs}$. This performance was ascribed due to stronger electronic and physical coupling in 2D/2D structure that provides more proficient electron transport (Fig. 28a-c). Also, Wee-Jun Ong et al. [303] studied 2D/2D sandwich-like graphene-g- $\mathrm{C}_{3} \mathrm{~N}_{4}(\mathrm{GCN})$ composite formed by one-pot impregnation thermal reduction technique. It also shows a high photo-catalytic activity for $\mathrm{CO}_{2}$ reduction to manufacture $\mathrm{CH}_{4}$. The large contact interface in graphene and $\mathrm{g}-\mathrm{C}_{3} \mathrm{~N}_{4}$ plays a significant function to improve photo-catalytic $\mathrm{CO}_{2}$ reduction by rising electron movement (Fig. 28d-f).

Furthermore, W. J. Ong et al. formed 2D/2D layered nano-structure of $\mathrm{rGO} / \mathrm{g}-\mathrm{C}_{3} \mathrm{~N}_{4}$ by surface charge modification and protonation for improved photo-catalytic reduction of $\mathrm{CO}_{2}$ to $\mathrm{CH}_{4}$ [305]. The preparations involved ultrasonic dispersion with $\mathrm{NaBH}_{4}$-reduction method (Fig. 29a). In comparison with pristine $\mathrm{g}-\mathrm{C}_{3} \mathrm{~N}_{4}$ and $\mathrm{rGO} / \mathrm{CN}$, optimized composite $15 \mathrm{wt} \% \mathrm{rGO} / \mathrm{pCN}(15 \mathrm{rGO} / \mathrm{pCN})$ showed high $\mathrm{CH}_{4}$ production of $13.93 \mathrm{mmol} \mathrm{g}^{-1}$ cat with a photochemical QY of $0.560 \%$ (Fig. 29b, c). Since $\mathrm{g}_{-} \mathrm{C}_{3} \mathrm{~N}_{4}$ also showed 2D p-conjugated structure, this quality improves the capability of catalyst to adsorb $\mathrm{CO}_{2}$ molecules. When electrostatically charged with $2 \mathrm{D}$ conducting material $\mathrm{rGO}, \mathrm{pCN}$, and $\mathrm{rGO}$ created efficient interface, which caused improved performance for $\mathrm{CO}_{2}$ reduction (Fig. 29d).

In addition, formation method of 2D/2D structure may affect photo-catalytic activity; for example, exfoliation production of graphene and $\mathrm{g}_{-} \mathrm{C}_{3} \mathrm{~N}_{4}$ will expose defects present on surface those are helpful for $\mathrm{CO}_{2}$ reduction, but surplus functional groups on catalyst surface will decrease conductance and number of active sites, therefore degrading activity. Therefore, Yu Teng Liang et al. [305] formed smaller quantity of surface defects that resulted in a higher 
Table 2 Comparison of current developments in 2D materials for solar-based $\mathrm{CO}_{2}$ reduction

\begin{tabular}{|c|c|c|c|}
\hline Photo-catalyst materials & $\mathrm{CO}_{2}$ reduction product & Average rate $\left(\mu \mathrm{mol} \mathrm{g}^{-1} \mathrm{~h}^{-1}\right)$ & References \\
\hline $\mathrm{TiO}_{2}$ NS-graphene & $\mathrm{CH}_{4}$ & 9.5 & [306] \\
\hline graphene- $\mathrm{Ti}_{0.91} \mathrm{O}_{2}$ hollow spheres & $\mathrm{CH}_{4} / \mathrm{CO}$ & $1.14 / 8.91$ & {$[307]$} \\
\hline $\mathrm{WO}_{3} \mathrm{NSs}$ & $\mathrm{CH}_{4}$ & 16 & {$[308]$} \\
\hline $\mathrm{MoS}_{2}-\mathrm{TiO}_{2}$ & $\mathrm{CH}_{4}$ & 10.6 & [309] \\
\hline $\mathrm{BiOCl}$ nano-plates & $\mathrm{CO}$ & 8.1 & [310] \\
\hline $\mathrm{BiOI} / \mathrm{g}-\mathrm{C}_{3} \mathrm{~N}_{4}$ & $\mathrm{CO} / \mathrm{CH}_{4}$ & $4.86 / 0.18$ & [311] \\
\hline $\mathrm{Ag}$ or $\mathrm{Au} / \mathrm{Zn}-\mathrm{Ga}-\mathrm{LDH}$ & $\mathrm{CO} / \mathrm{CH}_{3} \mathrm{OH}$ & $300 / 2010$ & [312] \\
\hline carbon-doped BN NSs & $\mathrm{CO}$ & 9.3 & {$[151]$} \\
\hline Sandwich-like graphene/g- $\mathrm{C}_{3} \mathrm{~N}_{4}$ & $\mathrm{CH}_{4}$ & 5.87 & [303] \\
\hline $\mathrm{rGO} /$ protonated g- $\mathrm{C}_{3} \mathrm{~N}_{4}$ & $\mathrm{CH}_{4}$ & 13.93 & {$[305]$} \\
\hline GO & $\mathrm{CH}_{3} \mathrm{OH}$ & 0.172 & [313] \\
\hline Boron-doped graphene (P25/B-GR) & $\mathrm{CH}_{4}$ & 2.5 & [314] \\
\hline $\begin{array}{l}\text { Oxygen-rich } \mathrm{TiO}_{2} \text {-doped graphene oxide (5GO- } \\
\mathrm{OTiO}_{2} \text { ) }\end{array}$ & $\mathrm{CH}_{4}$ & 3.450 & [315] \\
\hline $\mathrm{Cu}_{2} \mathrm{Se} /$ graphene & $\mathrm{CH}_{3} \mathrm{OH}$ & 2.63 & {$[316]$} \\
\hline rGO-TiO 2 & $\mathrm{CH}_{4}$ & 0.135 & [317] \\
\hline $\mathrm{Cu}_{2} \mathrm{O} / \mathrm{rGO}$ & $\mathrm{CH}_{3} \mathrm{OH}$ & 41.5 & [318] \\
\hline $\mathrm{CsPbBr}_{3} \mathrm{QDs} / \mathrm{GO}$ & $\mathrm{CH}_{4}$ & 29.6 & [319] \\
\hline $\mathrm{TiO}_{2}-\mathrm{CdS} / \mathrm{rGO}$ & $\mathrm{CH}_{4}$ & 0.115 & {$[320]$} \\
\hline $\mathrm{WO}_{3} \mathrm{NS}$ & $\mathrm{CH}_{4}$ & 16 & [308] \\
\hline $\mathrm{MoS}_{2} / \mathrm{Bi}_{2} \mathrm{WO}_{6}$ & $\mathrm{CH}_{3} \mathrm{OH}$ & 36.7 & {$[321]$} \\
\hline $\mathrm{MoS}_{2} / \mathrm{TiO}_{2}$ & $\mathrm{CH}_{4}$ & 10.6 & [309] \\
\hline $\mathrm{SnS}_{2}$ & $\mathrm{CO}$ & 12.28 & {$[322]$} \\
\hline $\mathrm{Bi}_{2} \mathrm{WO}_{6}$ & Methanol & 75 & [246] \\
\hline ZnAl-LDH NSs & $\mathrm{CO}$ & 7.6 & - \\
\hline $\mathrm{Vv}$-rich o-BiVO & Ethanol & 398.3 & - \\
\hline $\mathrm{Ti}_{3} \mathrm{C}_{2}$ with $\mathrm{P} 25$ & $\mathrm{CH}_{4}$ & & [252] \\
\hline
\end{tabular}

photo-catalytic $\mathrm{CO}_{2}$ reduction activity toward $\mathrm{CH}_{4}$ formation as compared to their counterparts with high quantity of surface defects. In this method, graphene was formed by two different techniques that involved rGO solvent-exfoliated graphene (SEG) way and coupled with $\mathrm{TiO}_{2}$ to make rGO$\mathrm{TiO}_{2}$ and SEG-TiO 2 , correspondingly. Due to its outstanding electron conductivity, $\mathrm{SEG}-\mathrm{TiO}_{2}$ showed higher photo-catalytic $\mathrm{CO}_{2}$ reduction activity compared to $\mathrm{rGO}-\mathrm{TiO}_{2}$. Hence, Table 2 shows the current developments in 2D materials for solar $\mathrm{CO}_{2}$ reduction devices.

\subsection{Photo-catalytic $\mathrm{H}_{2} \mathrm{O}_{2}$ Production}

Carbonaceous nanomaterials (NMs) with unique characteristics of $\mathrm{sp}^{2}$-hybridized carbon bonding with remarkable physicochemical nature at nanoscale usually show outstanding mechanical, chemical, and electrical properties [323]. In photo-catalysis research direction, carbonaceous NMs are frequently acted as $\mathrm{e}^{-}$transfer materials and photo-sensitizer that can widen adsorption edge and advance migration effectiveness of photo-induced $\mathrm{e}^{-} \mathrm{s}$ [324]. While carbonaceous NMs are immobilized on $\mathrm{g}-\mathrm{C}_{3} \mathrm{~N}_{4}$ photo-catalyst, they can accept and transport photo-induced $\mathrm{e}^{-} \mathrm{s}$ from $\mathrm{CB}$ level of $\mathrm{g}-\mathrm{C}_{3} \mathrm{~N}_{4}$ and boost the reduction reaction activity and so lead to superior photo-catalytic performance [325]. As typical carbonaceous NMs, CNTs with $\pi$-conjugative structure are able to accept, transport, and store $\mathrm{e}^{-} \mathrm{s}$ [326]. So, $\mathrm{g}^{-} \mathrm{C}_{3} \mathrm{~N}_{4}$-CNTs fabricated by incorporating the CNTs into $\mathrm{g}_{-} \mathrm{C}_{3} \mathrm{~N}_{4}$ can advance photocatalytic activity. Zhao and co-authors [327] applied the amination method to initiate CNTs in $\mathrm{g}-\mathrm{C}_{3} \mathrm{~N}_{4}$ NSs to make hybrid catalyst of $\mathrm{g}-\mathrm{C}_{3} \mathrm{~N}_{4} / \mathrm{CNTs}$, where CNTs were covalently mixed with g- $\mathrm{C}_{3} \mathrm{~N}_{4}$. The $\mathrm{g}-\mathrm{C}_{3} \mathrm{~N}_{4} / \mathrm{CNT}$ s hybrid photo-catalyst showed $\mathrm{H}_{2} \mathrm{O}_{2}$ formation rate of $32.6 \mu \mathrm{mol} \mathrm{h}^{-1}$ that was noticeably higher than $\mathrm{g}_{-} \mathrm{C}_{3} \mathrm{~N}_{4}\left(2.5 \mu \mathrm{mol} \mathrm{h}{ }^{-1}\right)$. The CNTs covalent 
combined with $\mathrm{g}_{-} \mathrm{C}_{3} \mathrm{~N}_{4}$ advanced $\mathrm{e}^{-}$generation via higher reduction capability and favorably shifted $\mathrm{CB}$ level to improve single $\mathrm{e}^{-}$reduction of $\mathrm{O}_{2}$ to $\cdot \mathrm{O}^{2-}$.

When polyoxometalates (POMs) [328] are irradiated through plentiful light energy, excitation of charge transfer from $\mathrm{O}^{2-}$ to $\mathrm{Mn}^{+}(n=5,6)$ is observed, guiding to development of $\mathrm{h}^{+}$center $\left(\mathrm{O}^{-}\right)$and trapped $\mathrm{e}^{-}$center $\left(\mathrm{M}_{(n)}^{+}\right)$pair. So, excited POMs can provide $\mathrm{e}^{-}$donors/acceptors. In addition, POMs have lesser recombination possibility of $\mathrm{e}^{-} \mathrm{s}$ and $\mathrm{h}^{+} \mathrm{s}$, as a result of its distinct HOMO-LUMO BGs. Taking advantages of these, POMs have been broadly used in photo-catalysts areas, for example $\mathrm{H}_{2} \mathrm{O}$ oxidation [329], $\mathrm{H}_{2}$-evolution [330], $\mathrm{CO}_{2}$-reduction [331], etc. Thus far, POMs also combined with g- $\mathrm{C}_{3} \mathrm{~N}_{4}$ for photo-catalytic $\mathrm{H}_{2} \mathrm{O}_{2}$ evolution. Zhao and co-workers [332] combined POM cluster of $\left[\mathrm{PW}_{11} \mathrm{O}_{39}\right]_{7}-\left(\mathrm{PW}_{11}\right)$ with $3 \mathrm{D}$ ordered macro-porous g- $\mathrm{C}_{3} \mathrm{~N}_{4}\left(3 \mathrm{DOM} \mathrm{g-} \mathrm{C}_{3} \mathrm{~N}_{4}\right.$ ) for efficient photo-catalytic $\mathrm{H}_{2} \mathrm{O}_{2}$ evolution. POM covalent cluster of $\mathrm{PW}_{11}$ was covalently bonded with $3 \mathrm{DOM} \mathrm{g}-\mathrm{C}_{3} \mathrm{~N}_{4}$ by captivating an organic linker approach. The quantity of synthesized $\mathrm{H}_{2} \mathrm{O}_{2}$ by g- $\mathrm{C}_{3} \mathrm{~N}_{4} /$ $\mathrm{PW}_{11}$ attained $3.5 \mu \mathrm{mol}$ within $60 \mathrm{~min}$, whereas catalytic activity of pristine $3 \mathrm{DOM} \mathrm{g-} \mathrm{C}_{3} \mathrm{~N}_{4}$ was only $1.3 \mu \mathrm{mol}$. The $\mathrm{CB}$ and $\mathrm{VB}$ of $3 \mathrm{DOM}$ g- $\mathrm{C}_{3} \mathrm{~N}_{4} / \mathrm{PW}_{11}$ were more positive compared to $3 \mathrm{DOM} \mathrm{g-} \mathrm{C}_{3} \mathrm{~N}_{4}$ that improved their potential for $\mathrm{H}_{2} \mathrm{O}$ oxidation and promoted $2 \mathrm{e}^{-}$reduction of $\mathrm{O}_{2}$ to $\mathrm{H}_{2} \mathrm{O}_{2}$. Similarly, they also studied a further POM cluster, $\left[\mathrm{SiW}_{11} \mathrm{O}_{39}\right] 8-\left(\mathrm{SiW}_{11}\right)$, to covalently combine with $\mathrm{g}-\mathrm{C}_{3} \mathrm{~N}_{4}$ [333].

Compared to $\mathrm{PW}_{11}$, the $\mathrm{SiW}_{11}$ possessed more negative $\mathrm{CB}$ level, helping $2 \mathrm{e}^{-}$reduction of $\mathrm{O}_{2}$ to $\mathrm{H}_{2} \mathrm{O}_{2}$. Under sunlight irradiation (AM 1.5), $\mathrm{H}_{2} \mathrm{O}_{2}$ photo-catalytic production of $15.2 \mu \mathrm{mol} \mathrm{h}{ }^{-1}$ over hybrid g- $\mathrm{C}_{3} \mathrm{~N}_{4} / \mathrm{SiW}_{11}$ photo-catalyst was attained. Except for direct combination of POMs with g- $\mathrm{C}_{3} \mathrm{~N}_{4}$, POMs-derived metal oxides were also incorporated with $\mathrm{g}-\mathrm{C}_{3} \mathrm{~N}_{4}$ to make hybrid photo-catalysts. Since POMs-derived metal oxides can accept, transport, and store $\mathrm{e}^{-} \mathrm{s}$, resulting hybrid photo-catalysts are able to improve photo-induced $\mathrm{e}^{-} \mathrm{s}$ generation and therefore increase performance of reduction reaction for $\mathrm{H}_{2} \mathrm{O}_{2}$ formation. Zhao and Zhao [334] studied g- $\mathrm{C}_{3} \mathrm{~N}_{4} / \mathrm{PWO}$ hybrid photo-catalyst through calcination of $\mathrm{g}-\mathrm{C}_{3} \mathrm{~N}_{4}$ precursor and $\left(\mathrm{NH}_{4}\right)_{3} \mathrm{PW}_{12} \mathrm{O}_{40}$ $\left(\mathrm{NH}_{4}-\mathrm{PW}_{12}\right)$ (POMs precursor). The hybrid g- $\mathrm{C}_{3} \mathrm{~N}_{4} / \mathrm{PWO}$ photo-catalyst showed competent photo-catalytic appearance for photo-catalytic $\mathrm{H}_{2} \mathrm{O}_{2}$ fabrication by vis-light in the absence of organic $\mathrm{e}^{-}$donor. In addition, a similar group [335] utilized another POMs-derived metal oxide to include g- $\mathrm{C}_{3} \mathrm{~N}_{4}$. The $\mathrm{g}-\mathrm{C}_{3} \mathrm{~N}_{4} / \mathrm{CoWO}$ hybrid photo-catalyst was fabricated via calcination of 3-amino 1, 2, 4-triazole (3-AT) and $\left(\mathrm{NH}_{4}\right)_{8} \mathrm{Co}_{2} \mathrm{~W}_{12} \mathrm{O}_{42}\left(\mathrm{NH}_{4}-\mathrm{Co}_{2} \mathrm{~W}_{12}\right)$. Under vis-light irradiation, $\mathrm{H}_{2} \mathrm{O}_{2}$ was rapidly generated over $\mathrm{g}-\mathrm{C}_{3} \mathrm{~N}_{4} / \mathrm{CoWO}$ and the amount of formed $\mathrm{H}_{2} \mathrm{O}_{2}$ was $18.7 \mu \mathrm{mol}$ in $60 \mathrm{~min}$, while individual g-C $3 \mathrm{~N} 4$ offered very lower photo-catalytic activity $(<0.1 \mu \mathrm{mol}$ in $60 \mathrm{~min})$. CoWO-incorporated $\mathrm{g}-\mathrm{C}_{3} \mathrm{~N}_{4}$ framework could produce more $\mathrm{e}^{-}$for $\mathrm{O}_{2}$ reduction, while negative shifts of $\mathrm{CB}$ level from $\mathrm{g}-\mathrm{C}_{3} \mathrm{~N}_{4}$ to g- $\mathrm{C}_{3} \mathrm{~N}_{4}$-CoWO enhanced single $\mathrm{e}^{-}$reduction of $\mathrm{O}_{2}$ to $\cdot \mathrm{O}^{2-}$. Also, incorporated CoWO advanced the oxidation of $\cdot \mathrm{O}^{2-}$ to $1 \mathrm{O}_{2}$ by $\mathrm{h}^{+}$ and formed $1 \mathrm{O}_{2}$ proceeded $2 \mathrm{e}^{-}$reduction to $\mathrm{H}_{2} \mathrm{O}_{2}$. Every one of these is related to enhanced photo-catalytic activity for $\mathrm{H}_{2} \mathrm{O}_{2}$ production over g- $\mathrm{C}_{3} \mathrm{~N}_{4} /$ CoWO hybrid photo-catalyst.

Organic counterparts have advantages of economic, simple formation and mechanical flexibility [336]. Mainly, organic photo-catalysts are capable to concentrate on faults of their inorganic counterparts, for example heavy metal with sensitive toxicity and restricted level of active sites. g- $\mathrm{C}_{3} \mathrm{~N}_{4}$ were recognized as representative organic polymer SC photo-catalysts. Recently, other organic SCs were also employed as photo-catalysts, e.g., triazine and aromatic diimides. Aromatic diimides possess high $\mathrm{e}^{-}$mobility and stability that are significant class of n-type organic SCs and incorporated with $\mathrm{g}-\mathrm{C}_{3} \mathrm{~N}_{4}$ for photo-catalytic $\mathrm{H}_{2} \mathrm{O}_{2}$ evolution. Shiraishi et al. [337] included facile way to aromatic diimide (pyromellitic diimide, PDI) in $\mathrm{g}_{-} \mathrm{C}_{3} \mathrm{~N}_{4}$ network by facile thermal condensation. These results showed that both $\mathrm{CB}$ and VB levels were developed into more positive via incorporation of PDI units in $\mathrm{g}_{-} \mathrm{C}_{3} \mathrm{~N}_{4}$ due to high $\mathrm{e}^{-}$affinity of PDI. This detail exposed that $\mathrm{g}-\mathrm{C}_{3} \mathrm{~N}_{4} / \mathrm{PDI}$ photo-catalyst has superior ability for $\mathrm{O}_{2}$ reduction; therefore, $\mathrm{H}_{2} \mathrm{O}_{2}$ evolution from $\mathrm{H}_{2} \mathrm{O}$ and $\mathrm{O}_{2}$ was promoted. Additionally, efficient synthesis of 1,4-endoperoxide species on photoexcited g- $\mathrm{C}_{3} \mathrm{~N}_{4} / \mathrm{PDI}$ suppressed $1 \mathrm{e}^{-}$reduction of $\mathrm{O}_{2}$ and $4 \mathrm{e}^{-}$reduction of $\mathrm{O}_{2}$, thus promoting selective two- $\mathrm{e}^{-}$reduction of $\mathrm{O}_{2}$ to $\mathrm{H}_{2} \mathrm{O}_{2}$.

Likewise, other categories of aromatic diimides were also incorporated with $\mathrm{g}-\mathrm{C}_{3} \mathrm{~N}_{4}$, for example biphenyl diimide (BDI) [338] and mellitic triimide (MTI) [339]. In pristine $\mathrm{H}_{2} \mathrm{O}$ with $\mathrm{O}_{2}$, both $\mathrm{g}-\mathrm{C}_{3} \mathrm{~N}_{4} / \mathrm{BDI}$ and $\mathrm{g}-\mathrm{C}_{3} \mathrm{~N}_{4} /$ MTI catalysts successfully produced millimolar levels of $\mathrm{H}_{2} \mathrm{O}_{2}$. For further activity improvement, rGO is incorporated with $\mathrm{g}_{-} \mathrm{C}_{3} \mathrm{~N}_{4} / \mathrm{PDI}$ catalyst [340] that takes advantage from 2D single-carbon monolayer property of rGO with high charge carrier mobility and high photochemical and 
thermal stability. The $\mathrm{g}_{-} \mathrm{C}_{3} \mathrm{~N}_{4} / \mathrm{PDI} / \mathrm{rGO}_{x}$ nano-hybrids photo-catalyst was synthesized by hydrothermal-calcination process. The photo-catalytic reaction testing showed that $\mathrm{g}_{-} \mathrm{C}_{3} \mathrm{~N}_{4} / \mathrm{PDI} / \mathrm{rGO}_{0.05}$ produced the largest amount of $\mathrm{H}_{2} \mathrm{O}_{2}(29 \mu \mathrm{mol})$ under vis-light irradiation within $24 \mathrm{~h}$, which was higher compared to $\mathrm{g}-\mathrm{C}_{3} \mathrm{~N}_{4}$ and g- $\mathrm{C}_{3} \mathrm{~N}_{4} / \mathrm{PDI}$. The SCC efficiency value of $\mathrm{g}-\mathrm{C}_{3} \mathrm{~N}_{4} / \mathrm{PDI} / \mathrm{rGO}_{0.05}$ was up to $0.20 \%$ that was higher compared to other counterparts. In this photo-catalyst system, rGO not only encouraged efficient charge division but also increased selective $2 \mathrm{e}^{-} \mathrm{O}_{2}$ reduction. The activity of g- $\mathrm{C}_{3} \mathrm{~N}_{4} / \mathrm{PDI}-\mathrm{rGO}$ photo-catalyst could be further improved by introducing $\mathrm{BN}$ because of spatial separation of $\mathrm{e}^{-}$and $\mathrm{h}^{+}$onto $\mathrm{rGO}$ and $\mathrm{BN}$, correspondingly [341]. Yang et al. [342] fabricated perylene imides (PI) on $\mathrm{g}-\mathrm{C}_{3} \mathrm{~N}_{4} \mathrm{NSs}$ to construct an all-solid-state Z-scheme hetero-junction. The hybrid g- $\mathrm{C}_{3} \mathrm{~N}_{4}$ /PI photocatalyst with Z-scheme arrangement promoted spatial separation of charge carriers, where photo-induced $\mathrm{e}^{-} \mathrm{s}$ in PI recombined with $\mathrm{h}^{+} \mathrm{s}$ in $\mathrm{g}-\mathrm{C}_{3} \mathrm{~N}_{4}$, while remaining $\mathrm{h}^{+} \mathrm{s}$ and $\mathrm{e}^{-} \mathrm{s}$ were left on PI and $\mathrm{g}-\mathrm{C}_{3} \mathrm{~N}_{4}$, correspondingly. Consequently, more $\mathrm{e}^{-} \mathrm{s}$ from $\mathrm{CB}$ of $\mathrm{g}-\mathrm{C}_{3} \mathrm{~N}_{4}$ part reduced $\mathrm{O}_{2}$ to produce more $\mathrm{H}_{2} \mathrm{O}_{2}$, while $\mathrm{h}^{+} \mathrm{s}$ of g- $\mathrm{C}_{3} \mathrm{~N}_{4} / \mathrm{PI}$ oxidized $\mathrm{OH}^{-}$to $\cdot \mathrm{OH}$ that also later reacted to generate $\mathrm{H}_{2} \mathrm{O}_{2}$. The shift of $\mathrm{H}_{2} \mathrm{O}_{2}$ production from single-channel to two-channel leads to significant enhancement in photo-catalytic $\mathrm{H}_{2} \mathrm{O}_{2}$ evolution. In another study, anthraquinone (AQ) was fastened on $\mathrm{g}-\mathrm{C}_{3} \mathrm{~N}_{4}$ surface that attains analogous roles as other organic SCs [343].

Fei Xue et al. [344] studied efficient photo-catalytic pure $\mathrm{H}_{2} \mathrm{O}_{2}$ splitting for simultaneous $\mathrm{H}_{2}$ and $\mathrm{H}_{2} \mathrm{O}_{2}$ fabrication. Photo-catalytic overall $\mathrm{H}_{2} \mathrm{O}$ splitting for instantaneous $\mathrm{H}_{2}$ and $\mathrm{H}_{2} \mathrm{O}_{2}$ generation via a $2 \mathrm{e}^{-}$pathway can readily address these issues. A novel $\mathrm{Co}_{x} \mathrm{Ni}_{y} \mathrm{P}$ cluster incorporated P-doped $\mathrm{g}_{-} \mathrm{C}_{3} \mathrm{~N}_{4}$ photo-catalyst $\left(\mathrm{Co}_{x} \mathrm{Ni}_{y} \mathrm{P}-\mathrm{PCN}\right)$ by two-step phosphating method that presents such unique behavior for pure $\mathrm{H}_{2} \mathrm{O}$ splitting into stoichiometric $\mathrm{H}_{2}$ and $\mathrm{H}_{2} \mathrm{O}_{2}$. The highest $\mathrm{H}_{2}$ production rate reaches $239.3 \mu \mathrm{mol} \mathrm{h}^{-1} \mathrm{~g}^{-1}$, achieved over CoNiP-PCN photo-catalyst that is among the best reported activities for overall $\mathrm{H}_{2} \mathrm{O}$ splitting. It is found that both $\mathrm{P}$ and cluster co-catalyst are critical to remarkably improved photo-catalytic activity. Specifically, $\mathrm{P}$ as a substitution of $\mathrm{C}$ in $\mathrm{PCN}$ introduces a positive charge center $\left(\mathrm{P}^{+}\right)$, reinforcing

Table 3 Summary of photo-catalytic $\mathrm{H}_{2} \mathrm{O}_{2}$ production

\begin{tabular}{|c|c|c|c|c|c|}
\hline Material & Sacrificial reagent & $\begin{array}{l}\text { Photo-catalyst con- } \\
\text { centration }\end{array}$ & Irradiation conditions & $\mathrm{H}_{2} \mathrm{O}_{2}$ yields & References \\
\hline $\mathrm{TiO}_{2}$ & $\mathrm{C}_{6} \mathrm{H}_{5} \mathrm{OH}$ & $10 \mathrm{mg} \mathrm{mL}^{-1}$ & $>280 \mathrm{~nm}$ & $40 \mathrm{mM}(12 \mathrm{~h})$ & {$[345]$} \\
\hline $\mathrm{TiO}_{2}$ & $2-\mathrm{C}_{3} \mathrm{H}_{7} \mathrm{OH}$ & $1 \mathrm{mg} \mathrm{mL}^{-1}$ & $365 \mathrm{~nm}$ & $423.2 \mu \mathrm{M}(2 \mathrm{~h})$ & [346] \\
\hline $\mathrm{Cu} / \mathrm{TiO}_{2}$ & - & $300 \mathrm{mg}$ & $300-400 \mathrm{~nm}$ & $8 \mu \mathrm{M}(5 \min )$ & [347] \\
\hline $\mathrm{F}-\mathrm{TiO}_{2}$ & $\mathrm{HCOOH}$ & $0.5 \mathrm{~g} \mathrm{~L}^{-1}$ & $360 \mathrm{~nm}$ & $1-1.3 \mathrm{mM}$ & [347] \\
\hline $\mathrm{Au} / \mathrm{TiO}_{2}$ & $\mathrm{HCOOH}$ & $1 \mathrm{mg} \mathrm{mL}^{-1}$ & $>420 \mathrm{~nm}$ & $640-700 \mu \mathrm{M}(1 \mathrm{~h})$ & [337] \\
\hline $\mathrm{CoPi} / \mathrm{rGO} / \mathrm{TiO}_{2}$ & $2-\mathrm{C}_{3} \mathrm{H}_{7} \mathrm{OH}$ & $0.5 \mathrm{~g} \mathrm{~L}^{-1}$ & $\geq 320 \mathrm{~nm}$ & $4.5 \mathrm{mM}(3 \mathrm{~h})$ & [348] \\
\hline $\mathrm{TiO}_{2} / \mathrm{WO}_{3} / \mathrm{rGO}$ & $2-\mathrm{C}_{3} \mathrm{H}_{7} \mathrm{OH}$ & $1 \mathrm{mg} \mathrm{mL}^{-1}$ & AM 1.5 & $\sim 270 \mu \mathrm{M}(1 \mathrm{~h})$ & [349] \\
\hline $\mathrm{Au} / \mathrm{TiO}_{2}$ & $\mathrm{CH}_{3} \mathrm{OH}$ & $1 \mathrm{mg} \mathrm{mL}^{-1}$ & $>320 \mathrm{~nm}$ & $1.06 \mathrm{mM}(3 \mathrm{~h})$ & {$[350]$} \\
\hline $\mathrm{Au} / \mathrm{SnO}_{2}-\mathrm{TiO}_{2}$ & Alcohol & $1 \mathrm{mg} \mathrm{mL}^{-1}$ & UV irradiation & $\sim 15 \mathrm{mM}(25 \mathrm{~h})-$ & {$[351]$} \\
\hline $\mathrm{g}-\mathrm{C}_{3} \mathrm{~N}_{4}$ & Alcohol & $4 \mathrm{mg} \mathrm{mL}^{-1}$ & $>420 \mathrm{~nm}$ & $30 \mu \mathrm{mol}(24 \mathrm{~h})$ & {$[345]$} \\
\hline Mesoporous g- $\mathrm{C}_{3} \mathrm{~N}_{4}$ & $\mathrm{EtOH}$ & $4 \mathrm{mg} \mathrm{mL}^{-1}$ & $>420 \mathrm{~nm}$ & $90 \mu \mathrm{mol}(24 \mathrm{~h})$ & {$[352]$} \\
\hline AQ-augmented $\mathrm{g}-\mathrm{C}_{3} \mathrm{~N}_{4}$ & $2-\mathrm{C}_{3} \mathrm{H}_{7} \mathrm{OH}$ & $0.5 \mathrm{mg} \mathrm{mL}^{-1}$ & AM 1.5 & $361 \mu \mathrm{mol}(1 \mathrm{~h})$ & {$[343]$} \\
\hline $\mathrm{KPF}_{6} / \mathrm{g}-\mathrm{C}_{3} \mathrm{~N}_{4}$ & $\mathrm{C}_{2} \mathrm{H}_{5}$ & $0.5 \mathrm{mg} \mathrm{mL}^{-1}$ & $>420 \mathrm{~nm}$ & $1.5 \mathrm{mM}(5 \mathrm{~h})$ & {$[353]$} \\
\hline Holey defective $\mathrm{g}-\mathrm{C}_{3} \mathrm{~N}_{4}$ & $\mathrm{H}_{2} \mathrm{O}+\mathrm{IPA}$ & $0.83 \mathrm{mg} \mathrm{mL}^{-1}$ & $>420 \mathrm{~nm}$ & $12.1 \mu \mathrm{mol}(2.5 \mathrm{~h})$ & {$[354]$} \\
\hline $\mathrm{O}_{2}$-enriched $\mathrm{g}-\mathrm{C}_{3} \mathrm{~N}_{4}$ & $\mathrm{H}_{2} \mathrm{O}+\mathrm{C}_{3} \mathrm{H}_{7} \mathrm{OH}$ & $1 \mathrm{mg} \mathrm{mL}^{-1}$ & $>420 \mathrm{~nm}$ & $300 \mu \mathrm{mol}(5 \mathrm{~h})$ & {$[355]$} \\
\hline $\mathrm{g}-\mathrm{C}_{3} \mathrm{~N}_{4}-\mathrm{SiW}_{11}$ & $\mathrm{CH}_{3} \mathrm{OH}$ & $1 \mathrm{mg} \mathrm{mL}^{-1}$ & AM 1.5 & $15.2 \mu \mathrm{mol}(1 \mathrm{~h})$ & {$[356]$} \\
\hline $\mathrm{g}-\mathrm{C}_{3} \mathrm{~N}_{4}-\mathrm{CoWO}$ & Organic $\mathrm{e}^{-}$ & $1 \mathrm{mg} \mathrm{mL}^{-1}$ & $\geq 420 \mathrm{~nm}$ & $9.7 \mu \mathrm{mol}(1 \mathrm{~h})$ & [357] \\
\hline$\left[\operatorname{RuII}\left(\mathrm{Me}_{2} \text { phen }\right)_{3}\right]^{2+}$ & $\mathrm{O}_{2}$-saturated $\mathrm{H}_{2} \mathrm{SO}_{4}$ & $1.0 \mu \mathrm{M}$ & $>420 \mathrm{~nm}$ & $612 \mu \mathrm{M}(9 \mathrm{~h})$ & [358] \\
\hline $\mathrm{Au} / \mathrm{BiVO}_{4}$ & $\mathrm{H}_{2} \mathrm{O}+\mathrm{EtOH}$ & $50 \mathrm{mg} / 30 \mathrm{~mL}$ & $>420 \mathrm{~nm}$ & $40.2 \mathrm{mM}(10 \mathrm{~h})$ & [359] \\
\hline $\mathrm{rGO} / \mathrm{Cd}_{3}(\mathrm{TMT})_{2}$ & $\mathrm{H}_{2} \mathrm{O}+\mathrm{C}_{2} \mathrm{H}_{5} \mathrm{OH}$ & $80 \mathrm{mg} / 20 \mathrm{~mL}$ & $>420 \mathrm{~nm}$ & $\sim 7 \mathrm{mM}(24 \mathrm{~h})$ & {$[360]$} \\
\hline Resins & $\mathrm{H}_{2} \mathrm{O}$ & $50 \mathrm{mg} / 30 \mathrm{~mL}$ & $>420 \mathrm{~nm}$ & $99 \mu \mathrm{mol}(24 \mathrm{~h})$ & [361] \\
\hline
\end{tabular}


chemical connection between $\mathrm{PCN}$ and $\mathrm{CoNiP}$, in the form of $\mathrm{P}^{+}-\mathrm{P}^{\delta-}-\mathrm{Co}^{\delta+} / \mathrm{Ni}^{\delta+}$. This unique bridging effect, together with extended light absorption by $\mathrm{P}$ doping and optimized surface redox potential by co-catalyst integration, stimulates efficient vectorial charge transfer between PCN and CoNiP and subsequent surface mass exchange. In contrast, this also shows that well-satisfied band structure of PCN can facilitate the $2 \mathrm{e}^{-}$reaction pathway, which not only has implication for potential use of CoNiP-PCN as potential photo-catalyst for solar $\mathrm{H}_{2}$ manufacture, and offers a new idea for pure $\mathrm{H}_{2} \mathrm{O}$ splitting in particulate system [344]. Table 3 offers intuitive summary of photo-catalytic-based $\mathrm{H}_{2} \mathrm{O}_{2}$ production.

\section{Future Perspective and Challenges}

In this review, a large number of experimental as well as theoretical research works related to photo-catalysts are discussed. A lot of confronts are required to be solved for incorporation of $2 \mathrm{D} p-n$ junctions in mass production electronic elements. Two most imperative confronts are scalable production of $2 \mathrm{D} p-\mathrm{n}$ junctions and environment degradation of 2DMs. Despite significant growth in ultrathin 2DMs for photo-catalysis, it is still suffering from large number of challenges in this direction. First, apart from extensively developed ultra-thin 2DMs, for instance $\mathrm{OH}$, MOs, and sulfides for photo-catalytic process, other types of probable ultra-thin 2DMs with novel structure or electronic properties for photo-catalysis should be investigated, for instance layer oxy-halides (e.g., $\mathrm{FeOBr}$ and $\mathrm{Bi}_{4} \mathrm{VO}_{8} \mathrm{Cl}$ ), thiophosphates (e.g., $\mathrm{CoPS}_{3}$ ), multi-metal chalcogenides (for instance $\mathrm{Cu}_{2} \mathrm{ZnGeS}_{4}$ ), or metal-free $\mathrm{SCs}$ (e.g., $\mathrm{C}_{3} \mathrm{~N}$ and $\mathrm{C}_{2} \mathrm{~N}$ ). Specially, ultra-thin $2 \mathrm{DMs}$ by means of intrinsic nonvdWs' layer structure have great potential in photo-catalysis, since plentiful surface atoms along dangling bonds can assist to construct outstanding chemical surroundings for supporting molecular reaction chemisorptions and enhance catalytic reaction dynamics. Second, there is an exceptional large-scale approach to yield ultra-thin 2D photo-catalysts with controlled thickness or crystal structures. The scalable manufacturing of ultra-thin 2D photo-catalysts has immense importance for possible commercial applications, and therefore more concentration should be given to cost-efficient scalable production approach. Third, atomicscale thicknesses permit ultra-thin materials along simply adjusting electronic structures that show an important effect on photo-catalytic performance. In spite of defect engineering, element doping, etc., or other efficient approaches for instance, engineering or tensile strain of surface state should be modified to engineer electronic structure and therefore enormously promote photo-catalytic activity. Fourth, ultrathin 2D arrangement offers a perfect material model to distinguish catalysts' active site, additional types of tuning, and reaction centers quantity to fulfill definite catalytic process requirement.

\subsection{Scalable Synthesis and Environmental Degradation}

First major confront is concerned with scalable synthesis of engineered vdWs hetero-structures with well-controlled edges. Still if deterministic assigned methods are flourishing at experimental level, they are not suitable for commercial purposes. Growth methods, such as CVD growth, have already been proven proficient for the growth of highquality 2DMs such as lateral and vertical hetero-structures at laboratory level. The VdWs epitaxial methods are even more promising for fabrication of high-quality $2 \mathrm{D}$ heterostructures. Upscale of such growth methods is probable, and the upcoming years will realize the application of higher-quality devices. A second, potential approach is to upscale growth of $2 \mathrm{D} p-n$ junctions to merge development of single 2DMs (e.g., $\mathrm{MoS}_{2}$ ) with different doping methods (frequently electrostatic or chemical). A second confront is environment degradation of numerous recognized 2DMs. For instance, while exposed to air, the BP in its ultra-thin structure is likely to absorb humidity that degrades material electronic properties. In context of BP, the most established degradation mechanism such as material reaction with $\mathrm{O}_{2}$ alters material properties. One method that prevents this degradation is encapsulating air-sensitive material among h-BN flakes under $\mathrm{O}^{2-}$ and humidity-free conditions. One active area of 2DMs investigation is consequently devoted to upscale encapsulation methods. A special move, which is at present practiced, is active investigation for novel $2 \mathrm{DMs}$ that did not create degradation problems, which could arrive either from the preparation (e.g., $\mathrm{TiS}_{3}$ ) or from natural resources (e.g., franckeite). Such, active search previously assisted to multiply number of present 2DMs only a handful more than twenty under 10 years. 


\subsection{Future Perspectives}

Other than traditional optoelectronic applications, 2D p-n junctions have still a lot of unknown applications and fundamental problems. For example, thermoelectric applications of $2 \mathrm{D}$ p-n junctions were not yet completely studied. The conventional Peltier device, a component mainly utilized in electronics for cooling (and commonly less for heating), depends on p- and n-type SC thermally coupled in parallel and series. The VdWs hetero-structures could be utilized to manufacture atomic-level thin cooling (or heating) elements in combination with other smart properties of 2DMs, e.g., high transparency or flexibility. Another exciting way is the study of new p-n junction geometries (e.g., circular p-n junctions in recent times showed in $G$ ) or novel devices based on 2D p-n junctions, e.g., logic gates or memories. Genuine probabilities still buried in 2DMs are in all possibilities than one described and 2D p-n junctions grasp much promise in larger-scale applications. Such 2D-junctions are particularly attractive building blocks of inflexible and transparent electronics, e.g., light-emitting diodes (LEDs) or solar cells. One more application, which can advantage from ultra-thin structure of $2 \mathrm{D} p-n$ junctions, is light-sensing and harvesting applications for nano-photonics. The $2 \mathrm{D} p-n$ junctions can be utilized as photodetectors, and numerous material combinations are present, which can be utilized to propose devices with sensible wavelengths ranging from infrared to UV that have already been established.

\section{Conclusion}

In recent years, new $\mathrm{p}-\mathrm{n}$ junctions manufacturing witnessed benefit of an ultra-thin nature of 2DMs. The top-down and bottom-up production methods have established competent generating $\mathrm{p}-\mathrm{n}$ junctions with high optoelectronic properties. 2DMs continue to offer numerous prospects to manufacture new p-n junctions with exceptional properties, which unlock motivating scientific directions both in requisites of elementary questions and with respect to applications [32]. Ultrathin 2DMs and their hybridizations through maintaining 2D arrangement are outstanding materials for elementary photo-catalytic investigation and promising marketable uses. This broad review highlights modern advancement in appliance of ultra-thin 2DMs for the photo-catalytic solar energy conversion. First, this review offers a complete outline of categorization and controlled fabrication mechanism of an ultra-thin 2D photo-catalyst. After that, approaches to modify electronic arrangement of ultra-thin2DMs and more effecting photo-catalytic properties are reviewed, that is, an element engineering, thickness engineering, defect engineering and doping. In addition, further hybridizations with upholding ultra-thin 2D characteristics are offered to further enhance photo-catalytic properties, such as QDs/2DMs, single atoms/2DMs, molecular/2DMs, and 2D-2D stacking materials. Lastly, a variety of photo-catalytic applications over ultra-thin 2D photo-catalysts were reviewed with emphasis on insights into structure-performance relationship, involving $\mathrm{H}_{2} \mathrm{O}$ oxidation, $\mathrm{H}_{2}$ evolution, $\mathrm{CO}_{2}$ reduction, $\mathrm{N}_{2}$ fixation, organic synthesis, and pollutants degradation. In conclusion, this review highlights universal approaches and current growth in 2D/2D hetero-junctions and hetero-structures that are outstanding candidates for basic investigation and possible catalyst applications because of their exclusive electronic structure and physicochemical properties:

1. Integrating their components' advantages, for instance ultra-thin 2D configuration, large surface area, and electronic/physicochemical properties;

2. Partition or charge transfer can be encouraged for required function; and

3. Versatile options (such as thickness, elements, defects, fabrication expertise, and contact space) can be designed to engineer properties and therefore application activity [136].

Heterogeneous photo-catalysis has turned into a fastgrowing galaxy with manifold miscellaneous matters being investigated and introduced. Driven through aforementioned benefits and possible standards of 2D/2D hetero-structures, increasing amount of extraordinary achievements were established in last few years. However, with challenges creating hurdles for real catalysis applications, e.g., catalyst competency, selectivity related to yield and pollution, environment friendly, and cost-efficient, there are still a lot of efforts to follow the purpose of developing required catalyst and reaction scheme for real-world applications. Catalysts grasp key for increasing effectiveness of catalytic reaction method which is the entrance of commercial practicable applications required to be prevailed over. The 2D/2D hetero-structures as photo-catalysts are planned with purpose to endorse light harvesting, charge carrier separation/transfer, redox reactivity, etc. In addition to sufficiently intriguing 
benefits of $2 \mathrm{D}$ elements via very well engineering of structure, composition, BG, and surface reaction sites, interfacial tuning at nanoscale of $2 \mathrm{D} / 2 \mathrm{D}$ hetero-structures is probable to further make easy photo-catalytic activity. Even though a lot of opportunities subsist for 2D/2D hetero-structures as electrocatalysts, this field is also facing a lot of challenges and research room:

1. Synthetic techniques are further required to be optimized to persuade commercial manufacturing demands.

2. Analysis techniques might be more superior for structure, physicochemical properties, and activity for these ultra-thin hetero-structures.

3. Clear comprehension of work mechanism, in particular reaction intermediates, of $2 \mathrm{D} / 2 \mathrm{D}$ hetero-junction electrocatalysts is still now required.

In addition, aforementioned optimization method of designing catalyst, high products yield/selectivity, and development of novel organic materials preparation are all solutions for further investigation. As a result of quick advancement and affluent information accumulated in the 2DMs and hetero-structures in last year's, one may anticipate that 2D/2D hetero-structures would participate in a significant role in resolving energy and environment confronts [136]. However, investigations concentrating on the photo-catalysis mechanism are still insufficient. A lot of hard work should be required to optimize the theoretical calculation setting, nearer to the industrial-scale practical reaction conditions. Parallel exploitation of theoretical approach along superior experimental approach can assist to get deeper understanding for connection between neighboring atomic microstructure and activity as well as elucidate reaction methods. Also, due to relevant limitations of every material, a preferred move to obtain optimal properties is performed to assemble diverse building blocks to produce supportive results. It is supposed that such a broad review will further put research in the field of 2DMs as a novel photo-catalysis [32].

Acknowledgements The research was partially supported by the National Natural Science Fund of China (Grant Nos. 61875138, 61435010, and 61961136001).

Open Access This article is licensed under a Creative Commons Attribution 4.0 International License, which permits use, sharing, adaptation, distribution and reproduction in any medium or format, as long as you give appropriate credit to the original author(s) and the source, provide a link to the Creative Commons licence, and indicate if changes were made. The images or other third party material in this article are included in the article's Creative Commons licence, unless indicated otherwise in a credit line to the material. If material is not included in the article's Creative Commons licence and your intended use is not permitted by statutory regulation or exceeds the permitted use, you will need to obtain permission directly from the copyright holder. To view a copy of this licence, visit http://creativecommons.org/licenses/by/4.0/.

\section{References}

1. K. Huang, Z. Li, J. Lin, G. Han, P. Huang, Two-dimensional transition metal carbides and nitrides (mxenes) for biomedical applications. Chem. Soc. Rev. 47, 5109-5124 (2018). https://doi.org/10.1039/C7CS00838D

2. K. Khan, A.K. Tareen, M. Aslam, K.H. Thebo, U. Khan et al., A comprehensive review on synthesis of pristine and doped inorganic room temperature stable mayenite electride, $\left[\mathrm{Ca}_{24} \mathrm{Al}_{28} \mathrm{O}_{64}\right]^{4+}\left(\mathrm{e}^{-}\right)_{4}$ and its applications as a catalyst. Prog. Solid State Chem. 54, 1-19 (2018). https://doi.org/10.1016/j. progsolidstchem.2018.12.001

3. K. Khan, A.K. Tareen, M. Aslam, A. Mahmood, Q. Khan et al., Going green with batteries and supercapacitor: two dimensional materials and their nanocomposites based energy storage applications. Prog. Solid State Chem. 34, 100254 (2019). https://doi.org/10.1016/j.progsolidstchem .2019 .100254

4. K. Khan, A.K. Tareen, M. Aslam, Y. Zhang, R. Wang, Z. Ouyang, Z. Gou, H. Zhang, Recent advances in two-dimensional materials and their nanocomposites in sustainable energy conversion applications. Nanoscale 11, 21622-21678 (2019). https://doi.org/10.1039/c9nr05919a

5. I. Dincer, Renewable energy and sustainable development: a crucial review. Renew. Sust. Energy Rev. 4, 157-175 (2000). https://doi.org/10.1016/S1364-0321(99)00011-8

6. X. Zou, Y. Zhang, Noble metal-free hydrogen evolution catalysts for water splitting. Chem. Soc. Rev. 44, 5148-5180 (2015). https://doi.org/10.1039/C4CS00448E

7. X. Chia, A.Y.S. Eng, A. Ambrosi, S.M. Tan, M. Pumera, Electrochemistry of nanostructured layered transition-metal dichalcogenides. Chem. Rev. 115, 11941-11966 (2015). https ://doi.org/10.1021/acs.chemrev.5b00287

8. H. Yin, Z. Tang, Ultrathin two-dimensional layered metal hydroxides: an emerging platform for advanced catalysis, energy conversion and storage. Chem. Soc. Rev. 45, 48734891 (2016). https://doi.org/10.1039/c6cs00343e

9. Y. Shi, H. Li, L.J. Li, Recent advances in controlled synthesis of two-dimensional transition metal dichalcogenides via vapour deposition techniques. Chem. Soc. Rev. 44, 27442756 (2015). https://doi.org/10.1039/C4CS00256C

10. Y. Jiao, Y. Zheng, M. Jaroniec, S.Z. Qiao, Design of electrocatalysts for oxygen-and hydrogen-involving energy conversion reactions. Chem. Soc. Rev. 44, 2060-2086 (2015). https ://doi.org/10.1039/c4cs00470a 
11. J. Pang, R.G. Mendes, A. Bachmatiuk, L. Zhao, H.Q. Ta et al., Applications of $2 \mathrm{~d}$ mxenes in energy conversion and storage systems. Chem. Soc. Rev. 48, 72-133 (2019). https ://doi.org/10.1039/C8CS00324F

12. H. Jin, C. Guo, X. Liu, J. Liu, A. Vasileff, Y. Jiao, Y. Zheng, S.Z. Qiao, Emerging two-dimensional nanomaterials for electrocatalysis. Chem. Rev. 118, 6337-6408 (2018). https://doi. org/10.1021/acs.chemrev.7b00689

13. Z. Zhang, E.S. Penev, B.I. Yakobson, Two-dimensional boron: structures, properties and applications. Chem. Soc. Rev. 46, 6746-6763 (2017)

14. Q. Meng, R.W. Xiu, J. Taeho, W. Miae, P.G. Young et al., Omnipotent phosphorene: a next-generation, two-dimensional nanoplatform for multidisciplinary biomedical applications. Chem. Soc. Rev. 47, 5588-5601 (2018). https://doi. org/10.1039/c8cs00342d

15. L. Dai, D.W. Chang, J.B. Baek, W. Lu, Carbon nanomaterials: carbon nanomaterials for advanced energy conversion and storage. Small 8, 1122 (2012). https://doi.org/10.1002/ smll.201101594

16. K. Khan, L. Jia, Z. Wenwei, X. Wei, Y. Ye, S. Weijie, Low temperature synthesis of nano porous $12 \mathrm{CaO} \cdot 7 \mathrm{Al}_{2} \mathrm{O}_{3}$ powder by hydrothermal method. J. Wuhan Univ. Technol.-Mater. Sci. Ed. 31, 1201-1205 (2016). https://doi.org/10.1007/s1159 5-016-1512-7

17. K. Khan, A.K. Tareen, S. Elshahat, A.K. Yadav, U. Khan et al., Facile synthesis of cationic doped $\left[\mathrm{Ca}_{24} \mathrm{Al}_{28} \mathrm{O}_{64}\right]^{4+}$. $\left(4 \mathrm{e}^{-}\right)$composite via rapid citrate sol-gel method. Dalton Trans. 47, 3819-3830 (2018). https://doi.org/10.1039/c7dt0 $4543 \mathrm{c}$

18. K. Khan, A.K. Tareen, J. Li, U. Khan, A. Nairan et al., Facile synthesis of tin-doped mayenite electride composite as a non-noble metal durable electrocatalyst for oxygen reduction reaction (ORR). Dalton Trans. 47, 13498-13506 (2018). https ://doi.org/10.1039/c8dt02548g

19. K. Khan, A.K. Tareen, M. Aslam, R. Wang, Y. Zhang et al., Recent developments in emerging two-dimensional materials and their applications. J. Mater. Chem. C 9, 1-9 (2019). https ://doi.org/10.1039/c9tc04187g

20. K. Khan, A.K. Tareen, M. Aslam, S.A. Khan, Q. Khan et al., Fe-doped mayenite electride composite with $2 d$ reduced graphene oxide: as a non-platinum based, highly durable electrocatalyst for oxygen reduction reaction. Sci. Rep. 9, 19809 (2019). https://doi.org/10.1038/s41598-019-55207-6

21. K. Khan, A.K. Tareen, M. Aslam, Q. Khan, S.A. Khan et al., Novel two-dimensional carbon-chromium nitride-based composite as an electrocatalyst for oxygen reduction reaction. Front. Chem. 7, 738 (2019). https://doi.org/10.3389/fchem .2019 .00738

22. K. Khan, A.K. Tareen, M. Aslam, R. Wang, Y. Zhang et al., Recent developments in emerging two-dimensional materials and their applications. J. Mater. Chem. C 8, 387-440 (2020). https://doi.org/10.1039/c9tc04187g

23. K. Khan, A.K. Tareen, S. Elshahat, N. Muhammad, J. Li et al., Facile metal-free reduction-based synthesis of pristine and cation-doped conductive mayenite. RSC Adv. 8,
24276-24285 (2018). https://doi.org/10.1039/c8ra02790k rsc.li/rsc-advances

24. K. Khan, A.K. Tareen, U. Khan, A. Nairan, S. Elshahat et al., Single step synthesis of highly conductive room-temperature stable cation-substituted mayenite electride target and thin film. Sci. Rep. (2018). https://doi.org/10.1038/s41598-01941512-7

25. A.K. Tareen, G.S. Priyanga, K. Khan, E. Pervaiz, T. Thomas, M. Yang, Nickel-based transition metal nitride electrocatalysts for the oxygen evolution reaction. Chemsuschem 12, 3941-3954 (2019). https://doi.org/10.1002/cssc.201900553

26. W. Zou, K. Khan, X. Zhao, C. Zhu, J. Huang et al., Direct fabrication of C12A7 electride target and room temperature deposition of thin films with low work function. Mater. Res. Express 4, 1-20 (2017). https://doi.org/10.1088/2053-1591/ aa63c7

27. K. Khan, A.K. Tareen, M. Aslam, M.F. Khan, Z. Shi et al., Synthesis, properties and novel electrocatalytic applications of the 2-D borophene xenes. Prog. Solid State Chem. 58, 100283 (2020). https://doi.org/10.1016/j.progsolidstchem .2020 .100283

28. W. Fan, Q. Zhang, Y. Wang, Semiconductor-based nanocomposites for photocatalytic $\mathrm{H}_{2}$ production and $\mathrm{Co}_{2}$ conversion. Phys. Chem. Chem. Phys. 15, 2632-2649 (2013). https://doi. org/10.1039/c2cp43524a

29. B. Luo, G. Liu, L. Wang, Recent advances in 2D materials for photocatalysis. Nanoscale 8, 6904-6920 (2016). https ://doi.org/10.1039/c6nr00546b

30. W.J. Zhang, W.W. Li, X.G. Chen, Z.G. Hu, W. Liu et al., Phonon mode and phase transition behaviors of $(1-\mathrm{x})$ $\mathrm{PbSc}_{1 / 2} \mathrm{Ta}_{1 / 2} \mathrm{O}_{3-\mathrm{x}} \mathrm{PbHfO}_{3}$ relaxor ferroelectric ceramics determined by temperature-dependent raman spectra. Appl. Phys. Lett. 99, 074103 (2011). https://doi. org/10.1063/1.3614431

31. R.D. Bonito, M.L. Elliott, E.A.D. Jardinm, Detection of an arbuscular mycorrhizal fungus in roots of different plant species with the PCR. Appl. Environ. Microb. 61, 2809-2810 (1995)

32. J. Di, J. Xiong, H. Li, Z. Liu, Ultrathin 2d photocatalysts: electronic-structure tailoring, hybridization, and applications. Adv. Mater. 30, 1-30 (2017). https://doi.org/10.1002/ adma.201704548

33. J. Ran, J. Zhang, J. Yu, M. Jaroniec, S.Z. Qiao, Earth-abundant cocatalysts for semiconductor-based photocatalytic water splitting. Chem. Soc. Rev. 46, 7787-7812 (2015). https ://doi.org/10.1039/C3CS60425J

34. Y. Ma, X. Wang, Y. Jia, X. Chen, H. Han, C. Li, Titanium dioxide-based nanomaterials for photocatalytic fuel generations. Chem. Rev. 114, 9987-10043 (2014). https://doi. org/10.1021/cr500008u

35. L.T.C. Equally, J. Zhang, L.Z. Li, N.A. Kumar, X.S. Zhao, Functionalization of chemically derived graphene for improving its electrocapacitive energy storage properties. Energy Environ. Sci. 9, 1891-1930 (2016). https://doi.org/10.1039/ C6EE00158K 
36. W.J. Ong, L.L. Tan, Y.H. Ng, S.T. Yong, S.P. Chai, Graphitic carbon nitride (g-C3N4)-based photocatalysts for artificial photosynthesis and environmental remediation: are we a step closer to achieving sustainability? Chem. Rev. 116, 71597329 (2016). https://doi.org/10.1021/acs.chemrev.6b00075

37. A. Fujishima, K. Honda, Electrochemical photolysis of water at a semiconductor electrode. Nature 238, 37-38 (1972). https ://doi.org/10.1038/238037a0

38. H.M. Tosine, J. Lawrence, J.H. Carey, Photodechlorination of PCB's in the presence of titanium dioxide in aqueous suspensions. B-Environ. Contam. Toxicol. 16, 697-701 (1976). https://doi.org/10.1007/BF01685575

39. X. Zhou, N. Liu, J. Schmidt, A. Kahnt, A. Osvet et al., Noble-metal-free photocatalytic hydrogen evolution activity: the impact of ball milling anatase nanopowders with $\mathrm{TiH}_{2}$. Adv. Mater. 29, 1604747 (2017). https://doi.org/10.1002/ adma.201604747

40. W.B. Choi, D.S. Chung, J.H. Kang, H.Y. Kim, Y.W. Jin et al., Fully sealed, high-brightness carbon-nanotube fieldemission display. Appl. Phys. Lett. 75, 3129 (1999). https:// doi.org/10.1063/1.125253

41. T. Hisatomi, K. Maeda, K. Takanabe, J. Kubota, K. Domen, Aspects of the water splitting mechanism on $(\mathrm{Ga} 1-\mathrm{xZnx})$ (n1-xOx) photocatalyst modified with Rh2-YCrYo3 cocatalyst. J. Phys. Chem. C 113, 21458-21466 (2009). https://doi. org/10.1021/jp9079662

42. T. Hisatomi, J. Kubota, K. Domen, Recent advances in semiconductors for photocatalytic and photoelectrochemical water splitting. Chem. Soc. Rev. 43, 7520-7535 (2014). https://doi. org/10.1039/C3CS60378D

43. Q. Bao, H. Zhang, B. Wang, Z. Ni, C.H.Y.X. Lim et al., Broadband graphene polarizer. Nat. Photonics 5, 411-415 (2011). https://doi.org/10.1038/nphoton.2011.102

44. W. Tao, N. Kong, X.Y. Ji, Y.P. Zhang, A. Sharma et al., Emerging two-dimensional monoelemental materials (Xenes) for biomedical applications. Chem. Soc. Rev. 48, 2891-2912 (2019). https://doi.org/10.1039/C8CS00823J

45. C. Tan, X. Cao, X.J. Wu, Q. He, J. Yang et al., Recent advances in ultrathin two-dimensional nanomaterials. Chem. Rev. 117, 6225-6331 (2017). https://doi.org/10.1021/acs. chemrev.6b00558

46. K.S. Novoselov, A.K. Geim, S.V. Morozov, D. Jiang, Y. Zhang et al., Electric field effect in atomically thin carbon films. Science 306, 666-669 (2004). https://doi.org/10.1126/ science. 1102896

47. Y. Sun, S. Gao, F. Lei, Y. Xie, Atomically-thin two-dimensional sheets for understanding active sites in catalysis. Chem. Soc. Rev. 44, 623-636 (2014). https://doi.org/10.1039/ C4CS00236A

48. Z. Du, S. Yang, S. Li, J. Lou, S. Zhang et al., Conversion of non-van der waals solids to $2 \mathrm{~d}$ transition-metal chalcogenides. Nature 577, 492-496 (2020). https://doi.org/10.1038/ s41586-019-1904-x

49. T. Su, Q. Shao, Z. Qin, Z. Guo, Z. Wu, Role of interfaces in two-dimensional photocatalyst for water splitting. ACS Catal.
8, 2253-2276 (2018). https://doi.org/10.1021/acscatal.7b034 37

50. J. Low, S. Cao, J. Yu, S. Wageh, Two-dimensional layered composite photocatalysts. Chem. Commun. 50, 10768-10777 (2014). https://doi.org/10.1039/C4CC02553A

51. S. Kouser, A. Thannikoth, U. Gupta, U.V. Waghmare, C.N.R. Rao, 2D-gas as a photocatalyst for water splitting to produce $\mathrm{H}_{2} \mathrm{O}_{2}$. Small 11, 4723-4730 (2015). https://doi.org/10.1002/ smll.201501077

52. Z. Chao, L. Wang, L. Gang, Q.L. Gao, H.M. Cheng, Template-free synthesis of $\mathrm{Ta}_{3} \mathrm{~N}_{5}$ nanorod arrays for efficient photoelectrochemical water splitting. Chem. Commun. 49, 3019-3021 (2013). https://doi.org/10.1039/C3CC40760H

53. N. Singh, G. Jabbour, U. Schwingenschlgl, Optical and photocatalytic properties of two-dimensional $\mathrm{MoS}_{2}$. Eur. Phys. J. B 85, 392-471 (2012). https://doi.org/10.1140/epjb/e2012 $-30449-7$

54. Q. Li, X. Li, S. Wageh, A.A. Al-Ghamdi, J. Yu, CdS/graphene nanocomposite photocatalysts. Adv. Energy Mater. 5, 1500010 (2015). https://doi.org/10.1002/aenm.201500010

55. Y. Li, Y.L. Li, B. Sa, R. Ahuja, Review of two-dimensional materials for photocatalytic water splitting from a theoretical perspective. Catal. Sci. Technol. 7, 545-559 (2017). https:// doi.org/10.1039/C6CY02178F

56. G.F. Chen, T.Y. Ma, Z.Q. Liu, N. Li, S.Z. Qiao, Efficient and stable bifunctional electrocatalysts $\mathrm{Ni} / \mathrm{Ni}_{\mathrm{x}} \mathrm{M}_{\mathrm{y}}(\mathrm{M}=\mathrm{P}$, S) for overall water splitting. Adv. Funct. Mater. 26, 3314 3323 (2016). https://doi.org/10.1002/adfm.201505626

57. M. Monai, M. Melchionna, P. Fornasiero, Chapter One - From metal to metal-free catalysts: Routes to sustainable chemistry. Adv. Catal. 63, 1-73 (2018). https://doi. org/10.1016/bs.acat.2018.10.001

58. T. Tian, Y. Li, D. Xie, Y. Shen, J. Ren et al., Clinical features and risk factors for post-partum depression in a large cohort of chinese women with recurrent major depressive disorder. J. Affect. Disord. 136, 983-987 (2012). https:// doi.org/10.1016/j.jad.2011.06.047

59. X. Li, M.W. Lin, J. Lin, B. Huang, A.A. Puretzky et al., Two-dimensional GaSe/MoSe ${ }_{2}$ misfit bilayer heterojunctions by van der waals epitaxy. Sci. Adv. 2, 1501882 (2016). https://doi.org/10.1126/sciadv.1501882

60. Q. Liang, L. Ye, Z.H. Huang, Q. Xu, Y. Bai, F. Kang, Q.H. Yang, A honeycomb-like porous carbon derived from pomelo peel for use in high-performance supercapacitors. Nanoscale 6, 13831-13837 (2014). https://doi.org/10.1039/ c4nr04541f

61. R. Kobayashi, S. Tanigawa, T. Takashima, B. Ohtani, H. Irie, Silver-inserted heterojunction photocatalysts for z-scheme overall pure-water splitting under visible-light irradiation. J. Phys. Chem. C 118, 22450-22456 (2014). https://doi.org/10.1021/jp5069973

62. B. Lin, H. Li, H. An, W. Hao, J. Wei et al., Preparation of $2 \mathrm{~d} / 2 \mathrm{~d} \mathrm{~g}-\mathrm{C}_{3} \mathrm{~N}_{4}$ nanosheet $@ \mathrm{ZNiN}_{2} \mathrm{~S}_{4}$ nanoleaf heterojunctions with well-designed high-speed charge transfer nanochannels towards high-efficiency photocatalytic hydrogen 
evolution. Appl. Catal. B Environ. 220, 542-552 (2018). https://doi.org/10.1016/j.apcatb.2017.08.071

63. H. Xiao, C. Tan, Z.Y.A. Zhang, 25th anniversary article: hybrid nanostructures based on two-dimensional nanomaterials. Adv. Mater. 26, 2185-2204 (2014). https://doi. org/10.1002/adma.201304964

64. Y.J. Wang, Y.M. Tao, F.Y. Li, Y.H. Wang, X.J. Xu et al., Pharmacological characterization of ATPM [(-)-3-aminothiazolo[5,4-b]-N-cyclopropylmethylmorphinan hydrochloride], a novel mixed $\kappa$-agonist and $\mu$-agonist/antagonist that attenuates morphine antinociceptive tolerance and heroin self-administration behavior. J. Pharmacol. Exp. Ther. 329, 306-313 (2009). https://doi.org/10.1124/ jpet.108.142802

65. J. Yang, D. Wang, H. Han, C. Li, Roles of cocatalysts in photocatalysis and photoelectrocatalysis. Acc. Chem. Res. 46, 1900-1909 (2013). https://doi.org/10.1021/ar300227e

66. M. Melchionna, P. Fornasiero, Updates on the roadmap for photocatalysis. ACS Catal. 10, 5493-5501 (2020). https:// doi.org/10.1021/acscatal.0c01204

67. B. Ohtani, Preparing articles on photocatalysis-beyond the illusions, misconceptions, and speculation. Chem. Lett. 37, 216-229 (2008). https://doi.org/10.1246/cl.2008.216

68. J. Zhang, Y. Huang, L. Jin, F. Rosei, F. Vetrone, J.P. Claverie, Efficient upconverting multiferroic core@ shell photocatalysts: visible-to-near-infrared photon harvesting. ACS Appl. Mater. Interfaces. 9, 8142-8150 (2017). https://doi. org/10.1021/acsami.7b00158

69. L. Wang, X. Xu, Q. Cheng, S.X. Dou, Y. Du, Near-infrareddriven photocatalysts: design, construction, and applications. Small (2019). https://doi.org/10.1002/smll.201904107

70. M. Freitag, N. Möller, A. Rühling, C.A. Strassert, B.J. Ravoo, F. Glorius, Photocatalysis in the dark: near-infrared light driven photoredox catalysis by an upconversion nanoparticle/photocatalyst system. ChemPhotoChem 3, 24-27 (2019). https://doi.org/10.1002/cptc.201800212

71. H. Chen, W. Liu, B. Hu, Z. Qin, H. Liu, A full-spectrum photocatalyst with strong near-infrared photoactivity derived from synergy of nano-heterostructured $\mathrm{Er}^{3+}$-doped multiphase oxides. Nanoscale 9, 18940-18950 (2017). https://doi. org/10.1039/C7NR08090E

72. S.E. Braslavsky, A.M. Braun, A.E. Cassano, A.V. Emeline, M.I. Litter et al., Glossary of terms used in photocatalysis and radiation catalysis. Pure Appl. Chem. 83, 931-1014 (2011). https://doi.org/10.1351/PAC-REC-09-09-36

73. M.J. Munoz-Batista, U. Caudillo-Flores, F. Ung-Medina, M. del Carmen Chávez-Parga, J.A. Cortés, A. Kubacka, M. Fernández-García, Gas phase 2-propanol degradation using titania photocatalysts: study of the quantum efficiency. Appl. Catal. B Environ. 201, 400-410 (2017). https://doi. org/10.1016/j.apcatb.2016.08.014

74. M. Shelef, R.W. McCabe, Twenty-five years after introduction of automotive catalysts: what next? Catal. Today 62, 35-50 (2000). https://doi.org/10.1016/S0920 $-5861(00) 00407-7$
75. S. Sui, X. Wang, X. Zhou, Y. Su, S. Riffat, C.-J. Liu, A comprehensive review of pt electrocatalysts for the oxygen reduction reaction: nanostructure, activity, mechanism and carbon support in PEM fuel cells. J. Mater. Chem. A 5, 1808-1825 (2017). https://doi.org/10.1039/C6TA08580F

76. J. Kou, C. Lu, J. Wang, Y. Chen, Z. Xu, R.S. Varma, Selectivity enhancement in heterogeneous photocatalytic transformations. Chem. Rev. 117, 1445-1514 (2017). https://doi. org/10.1021/acs.chemrev.6b00396

77. G.J. Ruiz-Mercado, R.L. Smith, M.A. Gonzalez, Sustainability indicators for chemical processes: i. Taxonomy. Ind. Eng. Chem. Res. 51, 2309-2328 (2012). https://doi.org/10.1021/ ie102116e

78. S.M. Fortier, N.T. Nassar, G.W. Lederer, J. Brainard, J. Gambogi, E.A. McCullough, Draft critical mineral list-summary of methodology and background information US geological survey technical input document in response to secretarial order no. 3359. 2018-1021 (2018). https://doi.org/10.3133/ ofr 20181021

79. A.L. Gulley, N.T. Nassar, S. Xun, China, the united states, and competition for resources that enable emerging technologies. Proc. Natl. Acad. Sci. U.S.A. 115, 4111-4115 (2018). https://doi.org/10.1073/pnas.1717152115

80. T. Paik, M. Cargnello, T.R. Gordon, S. Zhang, H. Yun et al., Photocatalytic hydrogen evolution from substoichiometric colloidal $\mathrm{WO}_{3-\mathrm{x}}$ nanowires. ACS Energy Lett. 3, 1904-1910 (2018). https://doi.org/10.1021/acsenergylett.8b00925

81. G. Carraro, C. Maccato, A. Gasparotto, T. Montini, S. Turner et al., Enhanced hydrogen production by photoreforming of renewable oxygenates through nanostructured $\mathrm{Fe}_{2} \mathrm{O}_{3}$ polymorphs. Adv. Funct. Mater. 24, 372-378 (2014). https://doi. org/10.1002/adfm.201302043

82. S. Qamar, F. Lei, L. Liang, S. Gao, K. Liu et al., Ultrathin $\mathrm{TiO}_{2}$ flakes optimizing solar light driven $\mathrm{CO}_{2}$ reduction. Nano Energy 26, 692-698 (2016). https://doi.org/10.1016/j. nanoen.2016.06.029

83. S. Gao, Y. Sun, F. Lei, J. Liu, L. Liang et al., Freestanding atomically-thin cuprous oxide sheets for improved visible-light photoelectrochemical water splitting. Nano Energy 8, 205-213 (2014). https://doi.org/10.1016/j.nanoe n.2014.05.017

84. F. Lei, Y. Sun, K. Liu, S. Gao, L. Liang, B. Pan, Y. Xie, Oxygen vacancies confined in ultrathin indium oxide porous sheets for promoted visible-light water splitting. J. Am. Chem. Soc. 136, 6826-6829 (2014). https://doi.org/10.1021/ ja501866r

85. J. Wang, C.J. Liu, Preparation of $2 \mathrm{D} \mathrm{WO}_{3}$ nanomaterials with enhanced catalytic activities: current status and perspective. Chembioeng. Rev. 2, 335-350 (2015). https://doi. org/10.1002/cben.201500014

86. L. Wang, T. Sasaki, Titanium oxide nanosheets: graphene analogues with versatile functionalities. Chem. Rev. 114, 9455-9486 (2014). https://doi.org/10.1021/cr400627u

87. N. Sakai, Y. Ebina, K. Takada, T. Sasaki, Photocurrent generation from semiconducting manganese oxide nanosheets in 
response to visible light. J. Phys. Chem. B 109, 9651-9655 (2005). https://doi.org/10.1021/jp0500485

88. R. Ma, T. Sasaki, Nanosheets of oxides and hydroxides: ultimate $2 \mathrm{~d}$ charge-bearing functional crystallites. Adv. Mater. 22, 5082-5104 (2011). https://doi.org/10.1002/adma.20100 1722

89. K. Akatsuka, G. Takanashi, Y. Ebina, M.A. Haga, T. Sasaki, Electronic band structure of exfoliated titanium- and/or niobium-based oxide nanosheets probed by electrochemical and photoelectrochemical measurements. J. Phys. Chem. C 116, 12426-12433 (2012). https://doi.org/10.1021/jp302417a

90. S. Ida, C. Ogata, M. Eguchi, W.J. Youngblood, T.E. Mallouk, Y. Matsumoto, Photoluminescence of perovskite nanosheets prepared by exfoliation of layered oxides, $\mathrm{K}_{2} \mathrm{Ln}_{2} \mathrm{Ti}_{3} \mathrm{O}_{10}$, $\mathrm{KLnNb}_{2} \mathrm{O}_{7}$, and $\mathrm{RbLnTa}_{2} \mathrm{O}_{7}$ (ln: lanthanide ion). J. Am. Chem. Soc. 39, 7052-7059 (2008). https://doi.org/10.1021/ ja7114772

91. K. Maeda, M. Eguchi, T. Oshima, Perovskite oxide nanosheets with tunable band-edge potentials and high photocatalytic hydrogen-evolution activity. Angew. Chem. Int. Ed. 46, 13164 (2015). https://doi.org/10.1002/anie.201408441

92. H. Yu, Q. Sun, X. Jia, X. Wang, J. Yu, Facile synthesis of porous $\mathrm{Bi}_{2} \mathrm{WO}_{6}$ nanosheets with high photocatalytic performance. Dalton Trans. 44, 14532-14539 (2015). https://doi. org/10.1039/C5DT01859E

93. E.L. Tae, K.E. Lee, J.S. Jeong, K.B. Yoon, Synthesis of diamond-shape titanate molecular sheets with different sizes and realization of quantum confinement effect during dimensionality reduction from two to zero. J. Am. Chem. Soc. 130, 6534-6543 (2008). https://doi.org/10.1021/ja711467g

94. Y. Zhou, Y. Zhang, M. Lin, J. Long, Z. Zhang et al., Monolayered $\mathrm{Bi}_{2} \mathrm{WO}_{6}$ nanosheets mimicking heterojunction interface with open surfaces for photocatalysis. Nat. Commun. 6, 8340 (2015). https://doi.org/10.1038/ncomms9340

95. J. Li, S. Qin, J. Xu, J. Xiong, C. Wu et al., Randomized, double-blind, placebo-controlled phase iii trial of apatinib in patients with chemotherapy-refractory advanced or metastatic adenocarcinoma of the stomach or gastroesophageal junction. J. Clin. Oncol. 34, 1448-1454 (2016). https://doi. org/10.1200/JCO.2015.63.5995

96. T. Zhang, C. Zhou, Y. Zhao, T. Bian, L. Shang et al., Bubble template synthesis of $\mathrm{Sn}_{2} \mathrm{Nb}_{2} \mathrm{O}_{7}$ hollow spheres for enhanced visible-light-driven photocatalytic hydrogen production. Chem. Commun. 49, 9872-9874 (2013). https ://doi.org/10.1039/c3cc45683h

97. Y. Zhao, G. Chen, T. Bian, C. Zhou, T. Zhang, Defectrich ultrathin $\mathrm{ZnAl}$-layered double hydroxide nanosheets for efficient photoreduction of $\mathrm{CO}_{2}$ to $\mathrm{CO}$ with water. Adv. Mater. 27, 7823 (2015). https://doi.org/10.1002/ adma. 201503730

98. Y.R. Liu, E.W. Loh, T.H. Lan, S.F. Chen, Y.H. Yu et al., ADRA1A gene is associated with BMI in chronic schizophrenia patients exposed to antipsychotics. Pharmacogenomics J. 10, 30-39 (2010). https://doi.org/10.1038/tpj.2009.55
99. Y. Zhao, B. Li, Q. Wang, W. Gao, C.J. Wang et al., NiTi-layered double hydroxides nanosheets as efficient photocatalysts for oxygen evolution from water using visible light. Chem. Sci. 5, 951-958 (2014). https://doi.org/10.1039/C3SC52546E

100. A. Hasani, M. Tekalgne, Q.V. Le, H.W. Jang, S.Y. Kim, Twodimensional materials as catalysts for solar fuels: hydrogen evolution reaction and $\mathrm{CO}_{2}$ reduction. J. Mater. Chem A 7, 430-454 (2019). https://doi.org/10.1039/C8TA09496A

101. Y. Xu, W. Zhao, R. Xu, Y. Shi, B. Zhang, Synthesis of ultrathin CdS nanosheets as efficient visible-light-driven water splitting photocatalysts for hydrogen evolution. Chem. Commun. 49, 9803-9805 (2013). https://doi.org/10.1039/ C3CC46342G

102. Q. He, C. Li, F. Geng, H. Yang, P. Li et al., Aerosol optical properties retrieved from sun photometer measurements over Shanghai, China. J. Geophys. Res. Atmos. 117, 16204 (2012). https://doi.org/10.1029/2011JD017220

103. H. Li, J. Wu, Z. Yin, H. Zhang, Preparation and applications of mechanically exfoliated single-layer and multi layer $\mathrm{MoS}_{2}$ and $\mathrm{WSe}_{2}$ nanosheets. ACS Chem. Res. 47, 1067-1075 (2014). https://doi.org/10.1021/ar4002312

104. J. Yu, C.Y. Xu, F.X. Ma, S.P. Hu, L. Zhen, Monodisperse sns ${ }_{2}$ nanosheets for high-performance photocatalytic hydrogen generation. ACS Appl. Mater. Interfaces. 6, 22370-22377 (2014). https://doi.org/10.1021/am506396z

105. C. Lin, X. Zhu, J. Feng, C. Wu, Y. Xie, Hydrogen-incorporated $\mathrm{TiS}_{2}$ ultrathin nanosheets with ultrahigh conductivity for stamp-transferrable electrodes. J. Am. Chem. Soc. 135, 5144-5151 (2013). https://doi.org/10.1021/ja400041f

106. Y.H. Sang, Z.H. Zhao, M.W. Zhao, P. Hao, Y.H. Leng, H. Liu, From UV to near-infrared, $\mathrm{WS}_{2}$ nanosheet: a novel photocatalyst for full solar light spectrum photodegradation. Adv. Mater. 27, 363-369 (2015). https://doi.org/10.1002/ adma.201403264

107. Y. Wu, M. Xu, X. Chen, S. Yang, H. Wu, J. Pan, X. Xiong, CTAB-assisted synthesis of novel ultrathin $\mathrm{MoSe}_{2}$ nanosheets perpendicular to graphene for adsorption and photodegradation of organic dyes under visible light. Nanoscale 8, 440450 (2015). https://doi.org/10.1039/C5NR05748E

108. S. Balendhran, S. Walia, H. Nili, J.Z. Ou, S. Zhuiykov et al., Semiconductors: two-dimensional molybdenum trioxide and dichalcogenides. Adv. Funct. Mater. 23, 3946 (2013). https:// doi.org/10.1002/adfm.201370159

109. M. Chhowalla, H.S. Shin, G. Eda, L.J. Li, K.P. Loh, H. Zhang, The chemistry of two-dimensional layered transition metal dichalcogenide nanosheets. Nat. Chem. 5, 263-275 (2013). https://doi.org/10.1038/nchem.1589

110. J.T. Jang, S. Jeong, J.W. Seo, M.C. Kim, E. Sim et al., Ultrathin zirconium disulfide nanodiscs. J. Am. Chem. Soc. 133, 7636-7639 (2011). https://doi.org/10.1021/ja200400n

111. J. Xie, J. Zhang, L. Shuang, F. Grote, X. Zhang et al., Correction to controllable disorder engineering in oxygen-incorporated $\mathrm{MoS}_{2}$ ultrathin nanosheets for efficient hydrogen evolution. J. Am. Chem. Soc. 136, 1680 (2014). https://doi. org/10.1021/ja4129636 
112. J. Hou, S. Cao, Y. Wu, F. Liang, L. Ye, Z. Lin, L. Sun, Perovskite-based nanocubes with simultaneously improved visible-light absorption and charge separation enabling efficient photocatalytic $\mathrm{CO}_{2}$ reduction. Nano Energy 30, 59-68 (2016). https://doi.org/10.1016/j.nanoen.2016.09.033

113. W.Q. Li, G. Wang, X.N. Zhang, H.P. Geng, J.L. Shen et al., Geometrical and morphological optimizations of plasmonic nanoarrays for high-performance SERS detection. Nanoscale (2015). https://doi.org/10.1039/C5NR03140K

114. M. Ablikim, S.S. Fang, H.X. Yang, M.G. Zhao, G.S. Varner et al., Observation of the decay $\psi(2 \mathrm{~S}) \rightarrow \mathrm{k}(892) \mathrm{k}^{-}+$c.c. Phys. Lett. B 614, 752-769 (2005). https://doi.org/10.1016/j.physl etb.2005.03.071

115. J. Di, J. Xia, Y. Huang, M. Ji, W. Fan, Z. Chen, H. Li, Constructing carbon quantum dots $/ \mathrm{Bi}_{2} \mathrm{SiO}_{5}$ ultrathin nanosheets with enhanced photocatalytic activity and mechanism investigation. Chem. Eng. J. 302, 334-343 (2016). https://doi. org/10.1016/j.cej.2016.05.009

116. H. Liu, F. Wang, L. Liu, X.Y. Jia, W. Zheng et al., Synthesis, characterization, and ethylene polymerization behaviors of late-transition metal complexes coordinated with chlorinated bis(arylimino)pyridine ligand. Polymer 55, 4611-4618 (2014). https://doi.org/10.1016/j.polymer.2014.07.015

117. L. Tan, X.C. Zhu, M.S. Tan, L. Sun, L. Tan et al., The genetic variation of ARRB2 is associated with late-onset Alzheimer's disease in Han Chinese. Curr. Alzheimer Res. 11, 408-412 (2014). https://doi.org/10.2174/15672050116661403170 95014

118. A. Hameed, T. Montini, V. Gombac, P. Fornasiero, Surface phases and photocatalytic activity correlation of $\mathrm{Bi}_{2} \mathrm{O}_{3}$ / $\mathrm{Bi}_{2} \mathrm{O}_{4-\mathrm{x}}$ nanocomposite. J. Am. Chem. Soc. 130, 9658-9659 (2008). https://doi.org/10.1021/ja803603y

119. M. Guan, C. Xiao, J. Zhang, S. Fan, Y. Xie, Vacancy associates promoting solar-driven photocatalytic activity of ultrathin bismuth oxychloride nanosheets. J. Am. Chem. Soc. 135, 10411-10417 (2013). https://doi.org/10.1021/ja402956f

120. J. Di, J.X. Xia, M.X. Ji, B. Wang, S. Yin et al., Advanced photocatalytic performance of graphene-like BN modified $\mathrm{BIOBr}$ flower-like materials for the removal of pollutants and mechanism insight. Appl. Catal. B Environ. 183, 254-262 (2016). https://doi.org/10.1016/j.apcatb.2015.10.036

121. D.R. Dreyer, S. Park, C.W. Bielawski, R.S. Ruoff, The chemistry of graphene oxide. Chem. Soc. Rev. 39, 228-240 (2010). https://doi.org/10.1039/b917103g

122. T.F. Yeh, J.M. Syu, C. Cheng, T.H. Chang, H. Teng, Graphite oxide as a photocatalyst for hydrogen production from water. Adv. Funct. Mater. 20, 2255-2262 (2010). https://doi. org/10.1002/adfm.201000274

123. J. Chu, J. Sun, L.I. Peng, L.I. Guangsheng, Y. Niu et al., Effect of platelet-rich plasma combined with human umbilical cord-mesenchymal stem cells on the healing of osteoporotic fracture in rats. Chin. J. Osteoporos. 135, 10411-10417 (2016). https://doi.org/10.1155/2016/9458396

124. Z. Zhou, J. Wang, J. Yu, Y. Shen, Y. Zhang, Dissolution and liquid crystals phase of $2 \mathrm{D}$ polymeric carbon nitride. J. Am.
Chem. Soc. 137, 2179-2182 (2015). https://doi.org/10.1021/ ja512179x

125. N. Ping, L. Zhang, G. Liu, H.M. Cheng, Graphene-like carbon nitride nanosheets for improved photocatalytic activities. Adv. Funct. Mater. 2, 4763-4770 (2012). https://doi. org/10.1002/adfm.201200922

126. S. Yang, Y. Gong, J. Zhang, Z. Liang, P.M.A. Jayan, Exfoliated graphitic carbon nitride nanosheets as efficient catalysts for hydrogen evolution under visible light. Adv. Mater. 25, 2452-2456 (2013). https://doi.org/10.1002/adma.201204453

127. C.X. Liu, Z.Y. Luo, Y.W. Li, M. Chen, J. Xu et al., Active waveguides by low-fluence carbon implantation in $\mathrm{Nd}^{3+}$-doped fluorophosphate glasses. Mod. Phys. Lett. B 30, 1550266 (2016). https://doi.org/10.1142/S02179849155026 68

128. H. Xu, J. Yan, X. She, L. Xu, J. Xia et al., Graphene-analogue carbon nitride: novel exfoliation synthesis and its application in photocatalysis and photoelectrochemical selective detection of trace amount of $\mathrm{Cu}^{2+}$. Nanoscale 6, 1406-1415 (2014). https://doi.org/10.1039/C3NR04759H

129. X. Zhang, X. Xie, H. Wang, J. Zhang, B. Pan, Y. Xie, Enhanced photoresponsive ultrathin graphitic-phase $\mathrm{C}_{3} \mathrm{~N}_{4}$ nanosheets for bioimaging. J. Am. Chem. Soc. 135, 18-21 (2013). https://doi.org/10.1021/ja308249k

130. I. Hevesi, L. Nánai, R. Vajtai, Laser light stimulated oxidation of vanadium at nonuniform illumination. Superlattice Microstruct. 3, 409-412 (1987). https://doi.org/10.1016/07496036(87)90214-X

131. H. Ou, L. Lin, Y. Zheng, P. Yang, Y. Fang, X. Wang, Tris-triazine-based crystalline carbon nitride nanosheets for an improved hydrogen evolution. Adv. Mater. 29, 1700008 (2017). https://doi.org/10.1002/adma.201700008

132. Z.T.Z. Tao, K.W.K. Wang, F.Y.F. Yi, C.Y.C. Yan, Q.L.Q. Li et al., A 3D soc design for H.264 application with onchip dram stacking, in 2010 IEEE International 3D Systems Integration Conference (3DIC), vol. 1, Corpus ID: 11735204 (2010). https://doi.org/10.1109/3DIC.2010.5751446

133. J. Ryu, Y.J. Jang, S. Choi, H.J. Kang, H. Park, J.S. Lee, S. Park, All-in-one synthesis of mesoporous silicon nanosheets from natural clay and their applicability to hydrogen evolution. NPG Asia Mater. 8, e248 (2016). https://doi. org/10.1038/am.2016.35

134. Y.P. Xie, J.-Y. Xing, X.-Y. Li, X. Wang, H.-J. Sun et al., Survey of sweetpotato viruses in China. Acta Virol. 57, 81-84 (2013). https://doi.org/10.4149/av_2013_01_81

135. O. Mashtalir, K.M. Cook, V.N. Mochalin, M. Crowe, M.W. Barsoum, Y. Gogotsi, Dye adsorption and decomposition on two-dimensional titanium carbide in aqueous media. J. Mater. Chem. A 2, 14334-14338 (2014). https://doi.org/10.1039/ C4TA02638A

136. J. Su, G.D. Li, X.H. Li, J.S. Chen, 2D/2D heterojunctions for catalysis. Adv. Sci. 6, 1801702 (2019). https://doi. org/10.1002/advs.201801702

137. K. Maeda, M. Eguchi, T. Oshima, Perovskite oxide nanosheets with tunable band-edge potentials and high photocatalytic hydrogen-evolution activity. Angew. Chem. Int. Ed. 
53, 13164-13168 (2014). https://doi.org/10.1002/anie.20140 8441

138. C.K. Ngaw, Q. Xu, T.T.Y. Tan, P. Hu, S. Cao, J.S.C. Loo, A strategy for in situ synthesis of well-defined core-shell Au@ $\mathrm{TiO}_{2}$ hollow spheres for enhanced photocatalytic hydrogen evolution. Chem. Eng. J. 257, 112-121 (2014). https://doi. org/10.1016/j.cej.2014.07.059

139. J. Li, G. Zhan, Y. Yu, L. Zhang, Superior visible light hydrogen evolution of Janus bilayer junctions via atomic-level charge flow steering. Nat. Commun. 7, 11480 (2016). https ://doi.org/10.1038/ncomms11480

140. Y. Wu, Z. Li, W. Ma, Y. Huang, L. Huo et al., PDT-S-T: a new polymer with optimized molecular conformation for controlled aggregation and $\pi-\pi$ stacking and its application in efficient photovoltaic devices. Adv. Mater. 25, 3449-3455 (2013). https://doi.org/10.1002/adma.201301174

141. E.Q. Chen, X.Q. Song, Y.L. Wang, T.Y. Zhou, L. Bai et al., Construction of a highly-active, liver-specific transcriptional regulatory element through combination of the albumin promoter and $\alpha$-fetoprotein enhancer. Plasmid 79, 87-88 (2011). https://doi.org/10.1016/j.plasmid.2010.11.006

142. S. Deckoff-Jones, J. Zhang, C.E. Petoukhoff, M.K.L. Man, S. Lei et al., Observing the interplay between surface and bulk optical nonlinearities in thin van der Waals crystals. Sci. Rep. 6, 22620 (2016). https://doi.org/10.1038/srep22620

143. Y. Sun, Z. Sun, S. Gao, H. Cheng, Q. Liu et al., Photoelectrochemical reactions: all-surface-atomic-metal chalcogenide sheets for high-efficiency visible-light photoelectrochemical water splitting. Adv. Energy Mater. 4, 1 (2014). https://doi. org/10.1002/aenm.201470002

144. Y. Sun, Z. Sun, S. Gao, H. Cheng, Q. Liu et al., All-surface-atomic-metal chalcogenide sheets for high-efficiency visible-light photoelectrochemical water splitting. Adv. Energy Mater. 4, 1300611 (2014). https://doi.org/10.1002/ aenm.201300611

145. D. Liang, H. Luo, Y.F. Liu, Z.Y. Hao, Y. Wang et al., Lysilactones A-C, three $6 \mathrm{H}$-dibenzo $(b, d)$ pyran-6-one glycosides from Lysimachia clethroides, total synthesis of lysilactone A. Tetrahedron 69, 2093-2097 (2013). https://doi.org/10.1016/j. tet.2013.01.029

146. Y. Li, Z. Wang, T. Xia, H. Ju, K. Zhang et al., Implementing metal-to-ligand charge transfer in organic semiconductor for improved visible-near-infrared photocatalysis. Adv. Mater. 28, 6959-6965 (2016). https://doi.org/10.1002/adma.20160 1960

147. F. Lei, L. Zhang, Y. Sun, L. Liang, K. Liu et al., Atomiclayer-confined doping for atomic-level insights into visiblelight water splitting. Angew. Chem. Int. Ed. 54, 9266-9270 (2015). https://doi.org/10.1002/ange.201503410

148. G. Liu, P. Niu, C.H. Sun, S.C. Smith, Z.G. Chen, G.Q. Lu, H.M. Cheng, Unique electronic structure induced high photoreactivity of sulfur-doped graphitic $\mathrm{C}_{3} \mathrm{~N}_{4}$. J. Am. Chem. Soc. 132, 11642-11648 (2010). https://doi.org/10.1021/ja103798k

149. J. Zhou, Y. Huang, X. Cao, B. Ouyang, W. Sun et al., Twodimensional $\mathrm{NiCo}_{2} \mathrm{O}_{4}$ nanosheet-coated three-dimensional graphene networks for high-rate, long-cycle-life supercapacitors. Nanoscale 7, 7035-7039 (2015). https://doi.org/10.1039/ C4NR06527A

150. Q. Qiao, B.H. Li, C.X. Shan, J.S. Liu, J. Yu et al., Light-emitting diodes fabricated from small-size $\mathrm{ZnO}$ quantum dots. Mater. Lett. 74, 104-106 (2012). https://doi.org/10.1016/j. matlet.2012.01.048

151. C. Huang, C. Chen, M. Zhang, L. Lin, X. Ye et al., Carbondoped BN nanosheets for metal-free photoredox catalysis. Nat. Commun. 6, 7698 (2015). https://doi.org/10.1038/ ncomms 8698

152. W. Bi, C. Ye, C. Xiao, W. Tong, X. Zhang, W. Shao, Y. Xie, Spatial location engineering of oxygen vacancies for optimized photocatalytic. Small 10, 2820-2825 (2014). https:// doi.org/10.1002/smll.201303548

153. B.E.S. Collaboration, M. Ablikim, J.Z. Bai, Y. Ban, J.G. Bian et al., Observation of the decay $(2 \mathrm{~S}) \mathrm{k}(892) \mathrm{k}+$ c.c. Phys. Lett. B 614, 37-43 (2005). https://doi.org/10.1016/j.phys1 etb.2005.03.071

154. S. Gao, B. Gu, X. Jiao, Y. Sun, X. Zu et al., Highly efficient and exceptionally durable $\mathrm{CO}_{2}$ photoreduction to methanol over freestanding defective single-unit-cell bismuth vanadate layers. J. Am. Chem. Soc. 139, 3438-3445 (2017). https:// doi.org/10.1021/jacs.6b11263

155. M. Cargnello, T. Montini, S.Y. Smolin, J.B. Prieb, J.J. Delgado Jaén et al., Engineering titania nanostructure to tune and improve its photocatalytic activity. Proc. Natl. Acad. Sci. 113, 3966-3971 (2016). https://doi.org/10.1073/pnas.1524806113

156. J. Du, M. Zhang, Z. Guo, J. Chen, X. Zhu et al., Phosphorene quantum dot saturable absorbers for ultrafast fiber lasers. Sci. Rep. 7, 42357 (2017). https://doi.org/10.1038/srep42357

157. Y. Ge, Z. Zhu, Y. Xu, Y. Chen, S. Chen et al., Broadband nonlinear photoresponse of $2 \mathrm{D} \mathrm{TiS}_{2}$ for ultrashort pulse generation and all-optical thresholding devices. Adv. Opt. Mater. 6, 1701166 (2018). https://doi.org/10.1002/adom.201701166

158. B. Guo, S.H. Wang, Z.X. Wu, Z.X. Wang, D.H. Wang et al., Sub-200 fs soliton mode-locked fiber laser based on bismuthene saturable absorber. Opt. Express 26, 22750 (2018). https ://doi.org/10.1364/oe.26.022750

159. X. Jiang, S. Liu, W. Liang, S. Luo, Z. He et al., Broadband nonlinear photonics in few-layer MXene $\mathrm{Ti}_{3} \mathrm{C}_{2} \mathrm{t}_{\mathrm{x}}(\mathrm{t}=\mathrm{F}, \mathrm{O}$, or OH). Laser Photonics Rev. 12, 1700229-1700239 (2018). https://doi.org/10.1002/lpor.201700229

160. X. Jiang, L. Zhang, S. Liu, Y. Zhang, Z. He et al., Ultrathin metal-organic framework: an emerging broadband nonlinear optical material for ultrafast photonics. Adv. Opt. Mater. 6, 1800561 (2018). https://doi.org/10.1002/adom.201800561

161. Y. Song, Z. Liang, X. Jiang, Y. Chen, Z. Li et al., Fewlayer antimonene decorated microfiber: ultra-short pulse generation and all-optical thresholding with enhanced long term stability. 2D Mater. 4, 045010 (2017). https://doi. org/10.1088/2053-1583/aa87c1

162. Z. Liu, H. Mu, S. Xiao, R. Wang, Z. Wang et al., Pulsed lasers employing solution-processed plasmonic $\mathrm{Cu}_{3-\mathrm{x}} \mathrm{P}$ colloidal nanocrystals. Adv. Mater. 28, 3535-3542 (2016). https ://doi.org/10.1002/adma.201504927 
163. X. Zhu, S. Chen, M. Zhang, L. Chen, Q. Wu et al., TiS ${ }_{2}$-based saturable absorber for ultrafast fiber laser. Photonics Res. 6, C44 (2018). https://doi.org/10.1364/prj.6.000c44

164. P. Li, Y. Chen, T. Yang, Z. Wang, H. Lin et al., Two-dimensional $\mathrm{CH}_{3} \mathrm{NH}_{3} \mathrm{PBi}_{3}$ perovskite nanosheets for ultrafast pulsed fiber lasers. ACS Appl. Mater. Interfaces. 9, 12759-12765 (2017). https://doi.org/10.1021/acsami.7b01709

165. M. Zhang, Q. Wu, F. Zhang, L. Chen, X. Jin et al., 2D black phosphorus saturable absorbers for ultrafast photonics. Adv. Opt. Mater. 7, 1800224 (2018). https://doi.org/10.1002/ adom.201800224

166. Y.F. Song, H. Zhang, D.Y. Tang, D.Y. Shen, Polarization rotation vector solitons in a graphene mode-locked fiber laser. Opt. Express 20, 27283-27289 (2012). https://doi. org/10.1364/OE.20.027283

167. Y. Chen, M. Wu, P. Tang, S. Chen, J. Du et al., The formation of various multi-soliton patterns and noise-like pulse in a fiber laser passively mode-locked by a topological insulator based saturable absorber. Laser Phys. Lett. 11, 055101 (2014). https://doi.org/10.1088/1612-2011/11/5/055101

168. Y. Ge, S. Chen, Y. Xu, Z. He, Z. Liang et al., Few-layer selenium-doped black phosphorus: synthesis, nonlinear optical properties and ultrafast photonics applications. J. Mater. Chem. C 5, 6129-6135 (2017). https://doi.org/10.1039/c7tc0 $1267 \mathrm{e}$

169. J. Li, H. Luo, B. Zhai, R. Lu, Z. Guo, H. Zhang, Y. Liu, Black phosphorus: a two-dimension saturable absorption material for mid-infrared q-switched and mode-locked fiber lasers. Sci. Rep. 6, 30361 (2016). https://doi.org/10.1038/srep30361

170. M. Liu, N. Zhao, H. Liu, X. Zheng, A. Luo et al., Dualwavelength harmonically mode-locked fiber laser with topological insulator saturable absorber. IEEE Photonics Technol. Lett. 26, 983-986 (2014). https://doi.org/10.1109/ lpt.2014.2311101

171. M. Liu, Z.R. Cai, S. Hu, A.P. Luo, C.J. Zhao et al., Dissipative rogue waves induced by long-range chaotic multi-pulse interactions in a fiber laser with a topological insulatordeposited microfiber photonic device. Opt. Lett. 40, 47674770 (2015). https://doi.org/10.1364/OL.40.004767

172. G. Zheng, Y. Chen, H. Huang, C. Zhao, S. Lu et al., Improved transfer quality of CVD-grown graphene by ultrasonic processing of target substrates: applications for ultra-fast laser photonics. ACS Appl. Mater. Interfaces. 5, 10288-10293 (2013). https://doi.org/10.1021/am403205v

173. Y.F. Song, H. Zhang, L.M. Zhao, D.Y. Shen, D.Y. Tang, Coexistence and interaction of vector and bound vector solitons in a dispersion-managed fiber laser mode locked by graphene. Opt. Express 24, 1814-1822 (2016). https:// doi.org/10.1364/OE.24.001814

174. Y. Song, S. Chen, Q. Zhang, L. Li, L. Zhao, H. Zhang, D. Tang, Vector soliton fiber laser passively mode locked by few layer black phosphorus-based optical saturable absorber. Opt. Express 24, 25933-25942 (2016). https:// doi.org/10.1364/OE.24.025933

175. Z.C. Luo, M. Liu, Z.N. Guo, X.F. Jiang, A.P. Luo et al., Microfiber-based few-layer black phosphorus saturable absorber for ultra-fast fiber laser. Opt. Express 23, 2003020039 (2015). https://doi.org/10.1364/OE.23.020030

176. Q. Wang, Y. Chen, L. Miao, G. Jiang, S. Chen et al., Wide spectral and wavelength-tunable dissipative soliton fiber laser with topological insulator nano-sheets self-assembly films sandwiched by PMMA polymer. Opt. Express 23, 7681-7693 (2015). https://doi.org/10.1364/OE.23.007681

177. Y. Xu, W. Wang, Y. Ge, H. Guo, X. Zhang et al., Stabilization of black phosphorous quantum dots in PMMA nanofiber film and broadband nonlinear optics and ultrafast photonics application. Adv. Funct. Mater. 27, 1702437 (2017). https://doi.org/10.1002/adfm.201702437

178. X.F. Jiang, Z. Zeng, S. Li, Z. Guo, H. Zhang, F. Huang, Q.H. Xu, Tunable broadband nonlinear optical properties of black phosphorus quantum dots for femtosecond laser pulses. Materials (Basel) 10, 210 (2017). https://doi. org/10.3390/ma10020210

179. Z. Wang, Y. Xu, S.C. Dhanabalan, J. Sophia, C. Zhao et al., Black phosphorus quantum dots as an efficient saturable absorber for bound soliton operation in an erbium doped fiber laser. IEEE Photonics J. 8, 1-10 (2016). https://doi. org/10.1109/jphot.2016.2598085

180. C. Ma, C. Wang, B. Gao, J. Adams, G. Wu, H. Zhang, Recent progress in ultrafast lasers based on $2 \mathrm{~d}$ materials as a saturable absorber. Appl. Phys. Rev. 6, 041304 (2019). https://doi.org/10.1063/1.5099188

181. T. Jiang, K. Yin, C. Wang, J. You, H. Ouyang et al., Ultrafast fiber lasers mode-locked by two-dimensional materials: review and prospect. Photonics Res. 8, 78 (2019). https:// doi.org/10.1364/prj.8.000078

182. Y. Song, X. Shi, C. Wu, D. Tang, H. Zhang, Recent progress of study on optical solitons in fiber lasers. Appl. Phys. Rev. 6, 021313 (2019). https://doi.org/10.1063/1.5091811

183. Y. Fang, Y. Ge, C. Wang, H. Zhang, Mid-infrared photonics using 2D materials: status and challenges. Laser Photonics Rev. 14, 1900098 (2019). https://doi.org/10.1002/lpor.20190 0098

184. J. Zheng, X. Tang, Z. Yang, Z. Liang, Y. Chen et al., Fewlayer phosphorene-decorated microfiber for all-optical thresholding and optical modulation. Adv. Opt. Mater. 5, 1700026 (2017). https://doi.org/10.1002/adom.201700026

185. J. Zheng, Z. Yang, C. Si, Z. Liang, X. Chen et al., Black phosphorus based all-optical-signal-processing: toward high performances and enhanced stability. ACS Photonics 4, 1466-1476 (2017). https://doi.org/10.1021/acsphotoni cs.7b00231

186. C. Wang, Y. Wang, X. Jiang, J. Xu, W. Huang et al., MXene $\mathrm{Ti}_{3} \mathrm{C}_{2} \mathrm{~T}_{\mathrm{x}}$ : a promising photothermal conversion material and application in all-optical modulation and all-optical information loading. Adv. Opt. Mater. 7, 1900060 (2019). https://doi. org/10.1002/adom.201900060

187. Y. Wang, W. Huang, J. Zhao, H. Huang, C. Wang et al., A bismuthene-based multifunctional all-optical phase and intensity modulator enabled by photothermal effect. J. Mater. Chem. C 7, 871-878 (2019). https://doi.org/10.1039/c8tc05513k 
188. L. Wu, W. Huang, Y. Wang, J. Zhao, D. Ma et al., 2d tellurium based high-performance all-optical nonlinear photonic devices. Adv. Funct. Mater. 29, 1806346 (2019). https://doi. org/10.1002/adfm.201806346

189. S. Chen, L. Miao, X. Chen, Y. Chen, C. Zhao et al., Few-layer topological insulator for all-optical signal processing using the nonlinear kerr effect. Adv. Opt. Mater. 3, 1769-1778 (2015). https://doi.org/10.1002/adom.201500347

190. Y. Song, Y. Chen, X. Jiang, Y. Ge, Y. Wang et al., Nonlinear few-layer MXene-assisted all-optical wavelength conversion at telecommunication band. Adv. Opt. Mater. 7, 1801777 (2019). https://doi.org/10.1002/adom.201801777

191. Y. Wang, F. Zhang, X. Tang, X. Chen, Y. Chen et al., Alloptical phosphorene phase modulator with enhanced stability under ambient conditions. Laser Photonics Rev. 12, 1800016 (2018). https://doi.org/10.1002/lpor.201800016

192. L. Wu, K. Chen, W. Huang, Z. Lin, J. Zhao et al., Perovskite $\mathrm{CSPBX}_{3}$ : a promising nonlinear optical material and its applications for ambient all-optical switching with enhanced stability. Adv. Opt. Mater. 6, 1800400 (2018). https://doi. org/10.1002/adom.201800400

193. L. Wu, Y. Dong, J. Zhao, D. Ma, W. Huang et al., Kerr nonlinearity in 2D graphdiyne for passive photonic diodes. Adv. Mater. 31, 1807981 (2019). https://doi.org/10.1002/ adma.201807981

194. L. Wu, X. Jiang, J. Zhao, W. Liang, Z. Li et al., Mxene-based nonlinear optical information converter for all-optical modulator and switcher. Laser Photonics Rev. 12, 1800215 (2018). https://doi.org/10.1002/lpor.201800215

195. L. Wu, Z. Xie, L. Lu, J. Zhao, Y. Wang et al., Few-layer tin sulfide: a promising black-phosphorus-analogue 2D material with exceptionally large nonlinear optical response, high stability, and applications in all-optical switching and wavelength conversion. Adv. Opt. Mater. 6, 1700985 (2018). https ://doi.org/10.1002/adom.201700985

196. Q. Wu, S. Chen, Y. Wang, L. Wu, X. Jiang et al., MZI-based all-optical modulator using mxene $\mathrm{Ti}_{3} \mathrm{C}_{2} \mathrm{~T}_{\mathrm{x}}(\mathrm{T}=\mathrm{F}$, O, or $\mathrm{OH})$ deposited microfiber. Adv. Mater. Technol. 4, 1800532 (2019). https://doi.org/10.1002/admt.201800532

197. Y. Wang, W. Huang, C. Wang, J. Guo, F. Zhang et al., An all-optical, actively q-switched fiber laser by an antimonenebased optical modulator. Laser Photonics Rev. 13, 1800313 (2019). https://doi.org/10.1002/lpor.201800313

198. Q. Ou, Y. Zhang, Z. Wang, J.A. Yuwono, R. Wang et al., Strong depletion in hybrid perovskite $\mathrm{p}-\mathrm{n}$ junctions induced by local electronic doping. Adv. Mater. 30, 1705792 (2018). https://doi.org/10.1002/adma.201705792

199. P. Guo, J. Xu, K. Gong, X. Shen, Y. Lu et al., On-nanowire axial heterojunction design for high-performance photodetectors. ACS Nano 10, 8474-8481 (2016). https://doi. org/10.1021/acsnano.6b03458

200. Z. Guo, S. Chen, Z. Wang, Z. Yang, F. Liu et al., Metal-ionmodified black phosphorus with enhanced stability and transistor performance. Adv. Mater. 29, 1703811 (2017). https:// doi.org/10.1002/adma.201703811
201. Z. Huang, W. Han, H. Tang, L. Ren, D.S. Chander, X. Qi, H. Zhang, Photoelectrochemical-type sunlight photodetector based on $\mathrm{MoS}_{2} /$ graphene heterostructure. 2D Mater. 2, 035011 (2015). https://doi.org/10.1088/2053-1583/2/3/03501 1

202. X. Ren, Z. Li, Z. Huang, D. Sang, H. Qiao et al., Environmentally robust black phosphorus nanosheets in solution: application for self-powered photodetector. Adv. Funct. Mater. 27, 1606834 (2017). https://doi.org/10.1002/adfm.201606834

203. Y. Xu, J. Yuan, K. Zhang, Y. Hou, Q. Sun et al., Field-induced n-doping of black phosphorus for CMOS compatible 2D logic electronics with high electron mobility. Adv. Funct. Mater. 27, 1702211 (2017). https://doi.org/10.1002/adfm.201702211

204. X. Ji, N. Kong, J. Wang, W. Li, Y. Xiao et al., A novel topdown synthesis of ultrathin 2D boron nanosheets for multimodal imaging-guided cancer therapy. Adv. Mater. 30, 1803031 (2018). https://doi.org/10.1002/adma.201803031

205. X. Liang, X. Ye, C. Wang, C. Xing, Q. Miao et al., Photothermal cancer immunotherapy by erythrocyte membrane-coated black phosphorus formulation. J. Control. Release 296, 150 161 (2019). https://doi.org/10.1016/j.jconrel.2019.01.027

206. M. Luo, T. Fan, Y. Zhou, H. Zhang, L. Mei, 2D black phosphorus-based biomedical applications. Adv. Funct. Mater. 29(13), 1808306 (2019). https://doi.org/10.1002/adfm.20180 8306

207. Z.B. Sun, Y.T. Zhao, Z.B. Li, H.D. Cui, Y.Y. Zhou et al., $\mathrm{TiL}_{4}$-coordinated black phosphorus quantum dots as an efficient contrast agent for in vivo photoacoustic imaging of cancer. Small 13, 1602896 (2017). https://doi.org/10.1002/ smll.201602896

208. M. Qiu, D. Wang, W. Liang, L. Liu, Y. Zhang et al., Novel concept of the smart NIR-light-controlled drug release of black phosphorus nanostructure for cancer therapy. Proc. Natl. Acad. Sci. 115, 501-506 (2018). https://doi. org/10.1073/pnas.1714421115

209. F. Yin, K. Hu, S. Chen, D. Wang, J. Zhang et al., Black phosphorus quantum dot based novel siRNA delivery systems in human pluripotent teratoma PA-1 cells. J. Mater. Chem. B 5, 5433-5440 (2017). https://doi.org/10.1039/ c7tb01068k

210. T. Fan, Y. Zhou, M. Qiu, H. Zhang, Black phosphorus: a novel nanoplatform with potential in the field of bio-photonic nanomedicine. J. Innov. Opt. Health Sci. 11, 1830003 (2018). https://doi.org/10.1142/s1793545818300033

211. W. Tao, X. Ji, X. Xu, M.A. Islam, Z. Li et al., Antimonene quantum dots: synthesis and application as near-infrared photothermal agents for effective cancer therapy. Angew. Chem. Int. Ed. 56, 11896-11900 (2017). https://doi.org/10.1002/ anie. 201703657

212. T. Xue, W. Liang, Y. Li, Y. Sun, Y. Xiang et al., Ultrasensitive detection of miRNA with an antimonene-based surface plasmon resonance sensor. Nat. Commun. 10, 28 (2019). https:// doi.org/10.1038/s41467-018-07947-8

213. H. Xie, Z. Li, Z. Sun, J. Shao, X.F. Yu et al., Metabolizable ultrathin $\mathrm{Bi}_{2} \mathrm{Se}_{3}$ nanosheets in imaging-guided 
photothermal therapy. Small 12, 4136-4145 (2016). https:// doi.org/10.1002/smll.201601050

214. W. Tao, X. Ji, X. Zhu, L. Li, J. Wang et al., Two-dimensional antimonene-based photonic nanomedicine for cancer theranostics. Adv. Mater. 30, 1802061 (2018). https://doi. org/10.1002/adma.201802061

215. J. Liu, Y. Liu, N. Liu, Y. Han, X. Zhang et al., Metal-free efficient photocatalyst for stable visible water splitting via a two-electron pathway. Science 347, 970-974 (2015). https:// doi.org/10.1126/science.aaa3145

216. J. Xia, J. Di, H. Li, H. Xu, H. Li, S. Guo, Ionic liquid-induced strategy for carbon quantum dots/BiOx $(\mathrm{x}=\mathrm{Br}, \mathrm{Cl})$ hybrid nanosheets with superior visible light-driven photocatalysis. Appl. Catal. B Environ. 181, 260-269 (2016). https://doi. org/10.1016/j.apcatb.2015.07.035

217. F. Xiang, S. Nan, Y. Liu, X. Chen, X. Zhou, Simultaneously enhanced stability and selectivity for propene epoxidation with $\mathrm{H}_{2}$ and $\mathrm{O}_{2}$ on Au catalysts supported on nano-crystalline mesoporous TS-1. ACS Catal. 7, 2668-2675 (2017). https:// doi.org/10.1021/acscatal.6b03498

218. B. Qiao, A. Wang, X. Yang, L.F. Allard, Z. Jiang et al., Single-atom catalysis of Co oxidation using $\mathrm{Pt}_{1} / \mathrm{FeO}_{\mathrm{x}}$. Nat. Chem. 3, 634-641 (2011). https://doi.org/10.1038/nchem .1095

219. Z. Chen, S. Pronkin, T.P. Fellinger, K. Kailasam, G. Vilé et al., Merging single-atom-dispersed silver and carbon nitride to a joint electronic system via copolymerization with silver tricyanomethanide. ACS Nano 10, 3166-3175 (2016). https://doi.org/10.1021/acsnano.5b04210

220. X. Li, W. Bi, L. Zhang, S. Tao, Y. Xie, Single-atom Pt as co-catalyst for enhanced photocatalytic $\mathrm{H}_{2}$ evolution. Adv. Mater. 28, 2427-2431 (2016). https://doi.org/10.1002/ adma.201505281

221. S. Ida, N. Kim, E. Ertekin, S. Takenaka, T. Ishihara, Photocatalytic reaction centers in two-dimensional titanium oxide crystals. J. Am. Chem. Soc. 137, 239-244 (2014). https:// doi.org/10.1021/ja509970z

222. Z. Han, F. Qiu, R. Eisenberg, P.L. Holland, T.D. Krauss, Robust photogeneration of $\mathrm{H}_{2}$ in water using semiconductor nanocrystals and a nickel catalyst. Science 338, 1321-1324 (2012). https://doi.org/10.1021/acscatal.5b02036

223. X. Lu, K. Xu, S. Tao, Z. Shao, X. Peng et al., Engineering the electronic structure of two-dimensional subnanopore nanosheets using molecular titanium-oxide incorporation for enhanced photocatalytic activity. Chem. Sci. 7, 14621467 (2016). https://doi.org/10.1039/C5SC03551A

224. Y.J. Yuan, Z.J. Ye, H. Lu, B. Hu, Y.H. Li et al., Constructing anatase $\mathrm{TiO}_{2}$ nanosheets with exposed (001) facets/ layered $\mathrm{MoS}_{2}$ two-dimensional nanojunction for enhanced solar hydrogen generation. ACS Catal. 6, 532-541 (2016). https://doi.org/10.1021/acscatal.5b02036

225. S. Ida, A. Takashiba, S. Koga, H. Hagiwara, T. Ishihara, Potential gradient and photocatalytic activity of an ultrathin $\mathrm{p}-\mathrm{n}$ junction surface prepared with two-dimensional semiconducting nanocrystals. J. Am. Chem. Soc. 136, 18721878 (2014). https://doi.org/10.1021/ja409465k
226. Y.Z. Huang, L.M. Wu, X.T. Wu, L.H. Li, L. Chen, Y.F. Zhang, $\mathrm{Pb}_{2} \mathrm{~B}_{5} \mathrm{O}_{9} \mathrm{I}$ : an iodide borate with strong second harmonic generation. J. Am. Chem. Soc. 132, 12788-12789 (2010). https://doi.org/10.1021/ja106066k

227. Y. Hou, A.B. Laursen, J. Zhang, G. Zhang, Y. Zhu et al., Layered nanojunctions for hydrogen-evolution catalysis. Angew. Chem. Int. Ed. 125, 3709-3713 (2013). https:// doi.org/10.1002/anie.201210294

228. Y.H. Huang, J.J. Wang, Z.M. Liu, G.D. Lin, H.B. Zhang, Highly efficient $\mathrm{Ni}-\mathrm{ZrO}_{2}$ catalyst doped with $\mathrm{YB}_{2} \mathrm{O}_{3}$ for comethanation of $\mathrm{CO}$ and $\mathrm{CO}_{2}$. Appl. Catal. A Gen. 466, 300306 (2013). https://doi.org/10.1016/j.apcata.2013.06.021

229. F. Wen, C. An, X. Wu, Y. Yang, J. Xu et al., MiR-34a regulates mitochondrial content and fat ectopic deposition induced by resistin through the AMPK/PPAR $\alpha$ pathway in HepG2 cells. Int. J. Biochem. Cell Biol. 94, 133-145 (2018). https://doi.org/10.1016/j.biocel.2017.11.008

230. J.L. Gunjakar, T.W. Kim, H.N. Kim, I.Y. Kim, S.J. Hwang, Mesoporous layer-by-layer ordered nanohybrids of layered double hydroxide and layered metal oxide: highly active visible light photocatalysts with improved chemical stability. J. Am. Chem. Soc. 133, 14998-15007 (2011). https:// doi.org/10.1021/ja203388r

231. Y. Sun, Z. Sun, S. Gao, H. Cheng, Q. Liu et al., Fabrication of flexible and freestanding zinc chalcogenide single layers. Nat. Commun. 3, 1057 (2012). https://doi.org/10.1038/ ncomms 2066

232. Y. Liu, L. Liang, C. Xiao, X. Hua, Z. Li, B. Pan, Y. Xie, Promoting photogenerated holes utilization in pore-rich $\mathrm{WO}_{3}$ ultrathin nanosheets for efficient oxygen-evolving photoanode. Adv. Energy Mater. 6, 1600437 (2016). https ://doi.org/10.1002/aenm.201600437

233. Y. Sun, Z. Sun, S. Gao, H. Cheng, Q. Liu et al., Fabrication of flexible and freestanding zinc chalcogenide single layers. Nat. Commun. 3, 1-7 (2012). https://doi.org/10.1038/ncomm s2066

234. Q. He, C. Li, F. Geng, H. Yang, P. Li et al., Aerosol optical properties retrieved from sun photometer measurements over Shanghai, China. J. Geophys. Res. Atmos. 117, 74-82 (2012). https://doi.org/10.1029/2011JD017220

235. Y. Sun, Z. Sun, S. Gao, H. Cheng, Q. Liu et al., Photoelectrochemical reactions: all-surface-atomic-metal chalcogenide sheets for high-efficiency visible-light photoelectrochemical water splitting. Adv. Energy Mater. 4, 1300574 (2014). https ://doi.org/10.1002/aenm.201470002

236. J. Zhu, Z. Yin, Y. Dan, T. Sun, Q. Yan, Hierarchical hollow spheres composed of ultrathin $\mathrm{Fe}_{2} \mathrm{O}_{3}$ nanosheets for lithium storage and photocatalytic water oxidation. Energy Environ. Sci. 6, 987-993 (2013). https://doi.org/10.1039/C2EE24148J

237. M. Ablikim, J.Z. Bai, Y. Bai, Y. Ban, X. Cai et al., Measurements of the observed cross sections for $\mathrm{e}^{+} \mathrm{e}^{-} \rightarrow$ exclusive light hadrons containing $\pi^{0} \pi^{0}$ at $\sqrt{ } \mathrm{s}=3.773,3.650$ and 3.6648 GeV. Phys. Lett. B 670, 179-183 (2008). https://doi. org/10.1016/j.physletb.2008.10.051

238. K.C. Kwon, S. Choi, K. Hong, D.M. Andoshe, J.M. Suh et al., Tungsten disulfide thin film/p-type si heterojunction 
photocathode for efficient photochemical hydrogen production. MRS Commun. 7, 272-279 (2017). https://doi. org/10.1002/anie.201210294

239. M. Zhang, J. Guan, Y. Tu, S. Chen, Y. Wang et al., Highly efficient $\mathrm{H}_{2}$ production from $\mathrm{H}_{2} \mathrm{~S}$ via a robust grapheneencapsulated metal catalyst. Energy Environ. Sci. 13, 119126 (2020). https://doi.org/10.1039/C9EE03231B

240. H. Wang, R. Peng, Z.D. Hood, M. Naguib, S.P. Adhikari, Z. $\mathrm{Wu}$, Titania composites with $2 \mathrm{D}$ transition metal carbides as photocatalysts for hydrogen production under visible-light irradiation. Chemsuschem 9, 1490-1497 (2016). https://doi. org/10.1002/cssc. 201600165

241. J. Peng, X. Chen, W.J. Ong, X. Zhao, N. Li, Surface and heterointerface engineering of 2D MXenes and their nanocomposites: insights into electro- and photocatalysis. Chem 5, 18-50 (2019). https://doi.org/10.1016/j.chempr.2018.08.037

242. T. Su, R. Peng, Z.D. Hood, M. Naguib, I.N. Ivanov, J.K. Keum, Z. Qin, Z. Guo, Z. Wu, One-step synthesis of $\mathrm{Nb}_{2} \mathrm{O}_{5} / \mathrm{C} / \mathrm{Nb}_{2} \mathrm{C}$ (MXene) composites and their use as photocatalysts for hydrogen evolution. Chemsuschem 11, 688 (2018). https://doi.org/10.1002/cssc.201702317

243. J. Ran, G. Gao, F.T. Li, T.Y. Ma, A. Du, S.Z. Qiao, $\mathrm{Ti}_{3} \mathrm{C}_{2}$ Mxene co-catalyst on metal sulfide photo-absorbers for enhanced visible-light photocatalytic hydrogen production. Nat. Commun. 8, 13907 (2017). https://doi.org/10.1038/ ncomms 13907

244. M. Shao, Y. Shao, J.W. Chai, Y. Qu, H. Pan, Synergistic effect of $2 \mathrm{D} \mathrm{Ti}{ }_{2} \mathrm{C}$ and $\mathrm{g}-\mathrm{C}_{3} \mathrm{~N}_{4}$ for efficient photocatalytic hydrogen production. J. Mater. Chem. A 5, 16748-16756 (2017). https ://doi.org/10.1039/C7TA04122E

245. A. Vasileff, C. Xu, Y. Jiao, Y. Zheng, S.Z. Qiao, Surface and interface engineering in copper-based bimetallic materials for selective $\mathrm{CO}_{2}$ electroreduction. Chem 4, 1809-1831 (2018). https://doi.org/10.1016/j.chempr.2018.05.001

246. L. Liang, F. Lei, S. Gao, Y. Sun, X. Jiao et al., Single unit cell bismuth tungstate layers realizing robust solar $\mathrm{CO}_{2}$ reduction to methanol. Angew. Chem. Int. Ed. 127, 14177-14180 (2015). https://doi.org/10.1002/anie.201506966

247. S. Asif, R. Kashif, N. Mohsin, M. Waheed, J. Jiseon et al., Heterostructural $\mathrm{TiO}_{2} / \mathrm{Ti}_{3} \mathrm{C}_{2} \mathrm{~T}_{\mathrm{x}}$ (MXene) for photocatalytic degradation of antiepileptic drug carbamazepine. Chem. Eng. J. 349, 748-755 (2017). https://doi.org/10.1021/jacs.6b11263

248. N. Li, X. Chen, W.J. Ong, D.R. MacFarlane, X. Zhao, A.K. Cheetham, C. Sun, Understanding of electrochemical mechanisms for $\mathrm{CO}_{2}$ capture and conversion into hydrocarbon fuels in transition-metal carbides (MXenes). ACS Nano 11, 1082510833 (2017). https://doi.org/10.1021/acsnano.7b03738

249. Y. Bai, L. Ye, T. Chen, L. Wang, X. Shi, X. Zhang, D. Chen, Facet-dependent photocatalytic $\mathrm{N}_{2}$ fixation of bismuth-rich $\mathrm{Bi}_{5} \mathrm{O}_{7 \mathrm{i}}$ nanosheets. ACS Appl. Mater. Interfaces. 8, 2766127668 (2016). https://doi.org/10.1021/acsami.6b08129

250. H. Li, J. Shang, Z. Ai, L. Zhang, Efficient visible light nitrogen fixation with $\mathrm{BIOBr}$ nanosheets of oxygen vacancies on the exposed 001 facets. J. Am. Chem. Soc. 137, 6393-6399 (2015). https://doi.org/10.1021/jacs.5b03105
251. S. Cao, B. Shen, T. Tong, J. Fu, J. Yu, 2D/2D heterojunction of ultrathin mxene $/ \mathrm{Bi}_{2} \mathrm{Wo}_{6}$ nanosheets for improved photocatalytic $\mathrm{CO}_{2}$ reduction. Adv. Funct. Mater. 28, 1800136 (2018). https://doi.org/10.1002/adfm.201800136

252. M. Ye, X. Wang, E. Liu, J. Ye, D. Wang, Boosting the photocatalytic activity of P25 for carbon dioxide reduction using a surface-alkalinized titanium carbide MXene as cocatalyst. Chemsuschem 11, 1606-1611 (2018). https://doi. org/10.1002/cssc. 201800083

253. C. Liu, Q. Xu, Q. Zhang, Y. Zhu, J. Xu, Layered BioBr/Ti ${ }_{3} \mathrm{C}_{2}$ MXene composite with improved visible-light photocatalytic activity. J. Mater. Sci. 54, 2458-2471 (2019). https://doi. org/10.1007/s10853-018-2990-0

254. X.Y. Kong, W.L. Tan, B.J. Ng, S.P. Chai, A.R. Mohamed, Harnessing Vis-NIR broad spectrum for photocatalytic $\mathrm{CO}_{2}$ reduction over carbon quantum dots-decorated ultrathin $\mathrm{Bi}_{2} \mathrm{Wo}_{6}$ nanosheets. Nano Res. 10, 1720-1731 (2017). https ://doi.org/10.1007/s12274-017-1435-4

255. J.R. Christianson, D. Zhu, R.J. Hamers, J.R. Schmidt, Mechanism of $\mathrm{N}_{2}$ reduction to $\mathrm{NH}_{3}$ by aqueous solvated electrons. J. Phys. Chem. B 118, 195-203 (2014). https://doi.org/10.1021/ jp406535p

256. H. Li, F. Qin, Z. Yang, X. Cui, J. Wang, L. Zhang, New reaction pathway induced by plasmon for selective benzyl alcohol oxidation on $\mathrm{BioCl}$ possessing oxygen vacancies. J. Am. Chem. Soc. 139, 3513-3521 (2017). https://doi.org/10.1021/ jacs.6b12850

257. N. Zhang, X. Li, H. Ye, S. Chen, H. Ju et al., Oxide defect engineering enables to couple solar energy into oxygen activation. J. Am. Chem. Soc. 138, 8928-8935 (2016). https:// doi.org/10.1021/jacs.6b04629

258. X.B. Li, C.H. Liu, R. Zhang, X.T. Huang, Y.Y. Li et al., Determination and pharmacokinetics of amygdalin in rats by LC-MS-MS. J. Chromatogr. Sci. 52, 476-481 (2013). https://doi.org/10.1093/chromsci/bmt063

259. C. Peng, X. Yang, Y. Li, H. Yu, H. Wang, F. Peng, Hybrids of two-dimensional $\mathrm{Ti}_{3} \mathrm{C}_{2}$ and $\mathrm{TiO}_{2}$ exposing 001 facets toward enhanced photocatalytic activity. ACS Appl. Mater. Interfaces. 8, 6051-6060 (2016). https://doi.org/10.1021/ acsami.5b11973

260. Z. Lin, D. Barbara, P.L. Taberna, K.L. Van Aken, B. Anasori, Y. Gogotsi, P. Simon, Capacitance of $\mathrm{Ti}_{3} \mathrm{C}_{2} \mathrm{t}_{\mathrm{x}}$ mxene in ionic liquid electrolyte. J. Power Sources 326, 575-579 (2016). https://doi.org/10.1016/j.jpows our.2016.04.035

261. X. Xie, N. Zhang, Z.R. Tang, M. Anpo, Y.J. Xu, $\mathrm{Ti}_{3} \mathrm{C}_{2} \mathrm{~T}_{\mathrm{x}}$ MXene as a janus cocatalyst for concurrent promoted photoactivity and inhibited photocorrosion. Appl. Catal. B Environ. 237, 43-49 (2018). https://doi.org/10.1016/j.apcat b.2018.05.070

262. H. Wang, Y. Wu, T. Xiao, X. Yuan, G. Zeng et al., Formation of quasi-core-shell $\mathrm{In}_{2} \mathrm{~S}_{3}$ /anatase $\mathrm{TiO}_{2} @$ metallic $\mathrm{Ti}_{3} \mathrm{C}_{2} \mathrm{t}_{\mathrm{x}}$ hybrids with favorable charge transfer channels for excellent visible-light-photocatalytic performance. Appl. Catal. B Environ. 233, 213-225 (2018). https://doi.org/10.1016/j. apcatb.2018.04.012 
263. J.M. Campos-Martin, G. Blanco-Brieva, J.L. Fierro, Hydrogen peroxide synthesis: an outlook beyond the anthraquinone process. Angew. Chem. Int. Ed. 45, 6962-6984 (2006). https ://doi.org/10.1002/anie.200503779

264. K. Sato, M. Aoki, R. Noyori, A "Green" Route to adipic acid: direct oxidation of cyclohexenes with 30 percent hydrogen peroxide. Science 281, 1646-1647 (1998). https://doi. org/10.1126/science.281.5383.1646

265. S. Yang, A. Verdaguer-Casadevall, L. Arnarson, L. Silvioli, V. Čolić et al., Toward the decentralized electrochemical production of $\mathrm{H}_{2} \mathrm{O}_{2}$ : a focus on the catalysis. ACS Catal. 8, 4064-4081 (2018). https://doi.org/10.1021/acscatal.8b00217

266. W. Zhan, L. Ji, Z.M. Ge, X. Wang, R.T. Li, A continuous-flow synthesis of primary amides from hydrolysis of nitriles using hydrogen peroxide as oxidant. Tetrahedron 74, 1527-1532 (2018). https://doi.org/10.1016/j.tet.2018.02.017

267. M. Ksibi, Chemical oxidation with hydrogen peroxide for domestic wastewater treatment. Chem. Eng. J. 119, 161-165 (2006). https://doi.org/10.1016/j.cej.2006.03.022

268. R.N. Gurram, M. Al-Shannag, N.J. Lecher, S.M. Duncan, E.L. Singsaas, M. Alkasrawi, Bioconversion of paper mill sludge to bioethanol in the presence of accelerants or hydrogen peroxide pretreatment. Bioresour. Technol. 192, 529-539 (2015). https://doi.org/10.1016/j.biortech.2015.06.010

269. K. Mase, M. Yoneda, Y. Yamada, S. Fukuzumi, Seawater usable for production and consumption of hydrogen peroxide as a solar fuel. Nat. Commun. 7, 1-7 (2016). https://doi. org/10.1038/ncomms 11470

270. Y.Q. Lan, X.L. Wang, L.Z. Dong, Q. Man, J.X. Su, Exploring the performance improvement of oxygen evolution reaction in stable bimetal-organic framework system. Angew. Chem. Int. Ed. 57, 9660-9664 (2018). https://doi.org/10.1002/ anie. 201803587

271. Z. Zhang, K. Liu, Z. Feng, Y. Bao, B. Dong, Hierarchical sheet-on-sheet $\mathrm{ZnIn}_{2} \mathrm{~S}_{4} / \mathrm{g}-\mathrm{C}_{3} \mathrm{~N}_{4}$ heterostructure with highly efficient photocatalytic $\mathrm{H}_{2}$ production based on photoinduced interfacial charge transfer. Sci. Rep. 6, 19221 (2016). https:// doi.org/10.1038/srep19221

272. C.Z. Liu, Y.F. Zhang, X.F. Li, X.F. Lu, Z. Chang et al., The high energy X-ray telescope (HE) onboard the insight-HXMT astronomy satellite. Sci. China Phys. Mech. 63, 249503 (2020). https://doi.org/10.1007/s11433-019-1486-X

273. M. Zhong, T. Hisatomi, Y. Kuang, J. Zhao, M. Liu et al., Surface modification of $\mathrm{CoO}_{x}$ loaded $\mathrm{BiVo}_{4}$ photoanodes with ultrathin p-type $\mathrm{NiO}$ layers for improved solar water oxidation. J. Am. Chem. Soc. 137, 5053-5060 (2015). https://doi. org/10.1021/jacs.5b00256

274. L. Wang, X. Zheng, L. Chen, Y. Xiong, H. Xu, Van der Waals heterostructures comprised of ultrathin polymer nanosheets for efficient z-scheme overall water splitting. Angew. Chem. Int. Ed. 130, 3512-3516 (2018). https://doi.org/10.1002/ ange. 201710557

275. M. Zhu, S. Kim, L. Mao, M. Fujitsuka, J. Zhang, X. Wang, T. Majima, Metal-free photocatalyst for $\mathrm{H}_{2}$ evolution in visible to near-infrared region: black phosphorus/graphitic carbon nitride. J. Am. Chem. Soc. 139, 13234-13242 (2017). https ://doi.org/10.1021/jacs.5b00256

276. J. Ran, W. Guo, H. Wang, B. Zhu, J. Yu, S.Z. Qiao, Metal-free 2D/2D phosphorene/g- $\mathrm{C}_{3} \mathrm{~N}_{4}$ van der Waals heterojunction for highly enhanced visible-light photocatalytic $\mathrm{h}_{2}$ production. Adv. Mater. 30, 1800128 (2018). https://doi.org/10.1002/ adma.201800128

277. Q. Gai, X. Zheng, W. Liu, Q. Dong, Y. Wang, R. Gao, S. Ren, 2D-2D heterostructured CdS-CoP photocatalysts for efficient H2 evolution under visible light irradiation. Int. J. Hydrog. Energy 44, 27412-27420 (2019). https://doi.org/10.1016/j. ijhydene.2019.08.196

278. R. Sasikala, A. Gaikwad, O. Jayakumar, K. Girija, R. Rao, A. Tyagi, S. Bharadwaj, Nanohybrid $\mathrm{MoS}_{2}$-PANI-CdS photocatalyst for hydrogen evolution from water. Colloids Surf. A 481, 485-492 (2015). https://doi.org/10.1016/j.colsu rfa.2015.06.027

279. M. Luo, W. Yao, C. Huang, Q. Wu, Q. Xu, Shape effects of pt nanoparticles on hydrogen production via $\mathrm{Pt} / \mathrm{CdS}$ photocatalysts under visible light. J. Mater. Chem A 3, 13884-13891 (2015). https://doi.org/10.1039/C5TA00218D

280. D. Lang, T. Shen, Q. Xiang, Roles of $\mathrm{MoS}_{2}$ and graphene as cocatalysts in the enhanced visible-light photocatalytic $\mathrm{H}_{2}$ production activity of multiarmed CdS nanorods. ChemCatChem 7, 943-951 (2015). https://doi.org/10.1002/ cctc. 201403062

281. W. Jiang, Y. Liu, R. Zong, Z. Li, W. Yao, Y. Zhu, Photocatalytic hydrogen generation on bifunctional ternary heterostructured $\mathrm{In}_{2} \mathrm{~S}_{3} / \mathrm{MoS}_{2} / \mathrm{CdS}$ composites with high activity and stability under visible light irradiation. J. Mater. Chem. A 3, 18406-18412 (2015). https://doi.org/10.1039/C5TA04258E

282. T. Jia, A. Kolpin, C. Ma, R.C.T. Chan, W.M. Kwok, S.E. Tsang, A graphene dispersed CdS- $\mathrm{MoS}_{2}$ nanocrystal ensemble for cooperative photocatalytic hydrogen production from water. Chem. Commun. 50, 1185-1188 (2014). https://doi. org/10.1039/C3CC47301E

283. K. Chang, Z. Mei, T. Wang, Q. Kang, S. Ouyang, J. Ye, $\mathrm{MoS}_{2}$ /graphene cocatalyst for efficient photocatalytic $\mathrm{H}_{2}$ evolution under visible light irradiation. ACS Nano 8, 70787087 (2014). https://doi.org/10.1021/nn5019945

284. K. Chang, M. Li, T. Wang, S. Ouyang, P. Li, L. Liu, J. Ye, Drastic layer number dependent activity enhancement in photocatalytic $\mathrm{H}_{2}$ evolution over $\mathrm{nMoS}_{2} / \mathrm{CdS}(\mathrm{n} \geq 1)$ under visible light. Adv. Energy Mater. 5, 1402279 (2015). https:// doi.org/10.1021/nn5019945

285. X. Zong, G. Wu, H. Yan, G. Ma, J. Shi et al., Photocatalytic $\mathrm{H}_{2}$ evolution on $\mathrm{MoS}_{2} / \mathrm{CdS}$ catalysts under visible light irradiation. J. Phys. Chem. C 114, 1963-1968 (2010). https://doi. org/10.1021/jp904350e

286. X. Zong, H. Yan, G. Wu, G. Ma, F. Wen, L. Wang, C. Li, Enhancement of photocatalytic $\mathrm{H}_{2}$ evolution on CdS by loading $\mathrm{MoS}_{2}$ as cocatalyst under visible light irradiation. J. Am. Chem. Soc. 130, 7176-7177 (2008). https://doi.org/10.1021/ ja8007825

287. S. Ma, J. Xie, J. Wen, K. He, X. Li, W. Liu, X. Zhang, Constructing 2D layered hybrid CdS nanosheets/mos ${ }_{2}$ 
heterojunctions for enhanced visible-light photocatalytic $\mathrm{H}_{2}$ generation. Appl. Surf. Sci. 391, 580-591 (2017). https://doi. org/10.1016/j.apsusc.2016.07.067

288. X. Wang, K. Maeda, A. Thomas, K. Takanabe, G. Xin et al., A metal-free polymeric photocatalyst for hydrogen production from water under visible light. Nat. Mater. 8, 76-80 (2009). https://doi.org/10.1038/nmat2317

289. L. Jia, D.H. Wang, Y.X. Huang, A.W. Xu, H.Q. Yu, Highly durable n-doped graphene/cds nanocomposites with enhanced photocatalytic hydrogen evolution from water under visible light irradiation. J. Phys. Chem. C 115, 11466-11473 (2011). https://doi.org/10.1021/jp2023617

290. U. Maitra, U. Gupta, M. De, R. Datta, A. Govindaraj, C. Rao, Highly effective visible-light-induced $\mathrm{H}_{2}$ generation by single-layer $1 \mathrm{~T}-\mathrm{MoS}_{2}$ and a nanocomposite of few-layer $2 \mathrm{H}-\mathrm{MoS}_{2}$ with heavily nitrogenated graphene. Angew. Chem. Int. Ed. 52, 13057-13061 (2013). https://doi.org/10.1002/ anie. 201306918

291. U. Gupta, B. Naidu, U. Maitra, A. Singh, S.N. Shirodkar, U.V. Waghmare, C. Rao, Characterization of few-layer 1T-MoSe ${ }_{2}$ and its superior performance in the visible-light induced hydrogen evolution reaction. APL Mater. 2, 092802 (2014). https://doi.org/10.1063/1.4892976

292. B. Mahler, V. Hoepfner, K. Liao, G.A. Ozin, Colloidal synthesis of $1 \mathrm{~T}-\mathrm{WS}_{2}$ and $2 \mathrm{H}-\mathrm{WS}_{2}$ nanosheets: applications for photocatalytic hydrogen evolution. J. Am. Chem. Soc. 136, 14121-14127 (2014). https://doi.org/10.1021/ja506261t

293. J. Chen, X.J. Wu, L. Yin, B. Li, X. Hong et al., One-pot synthesis of CdS nanocrystals hybridized with single-layer transition-metal dichalcogenide nanosheets for efficient photocatalytic hydrogen evolution. Angew. Chem. Int. Ed. 54, 1210-1214 (2015). https://doi.org/10.1002/anie.201410172

294. Z. Lei, W. You, M. Liu, G. Zhou, T. Takata et al., Photocatalytic water reduction under visible light on a novel $\mathrm{ZnIn}_{2} \mathrm{~S}_{4}$ catalyst synthesized by hydrothermal method. Chem. Commun. 17, 2142-2143 (2003). https://doi.org/10.1039/B3068 $13 \mathrm{G}$

295. Z. Xu, Y. Li, S. Peng, G. Lu, S. Li, Nacl-assisted low temperature synthesis of layered $\mathrm{Zn}-\mathrm{In}-\mathrm{S}$ photocatalyst with high visible-light activity for hydrogen evolution. RSC Adv. 2, 3458-3466 (2012). https://doi.org/10.1039/C2RA01159J

296. J. Yu, L. Qi, M. Jaroniec, Hydrogen production by photocatalytic water splitting over $\mathrm{Pt} / \mathrm{TiO}_{2}$ nanosheets with exposed (001) facets. J. Phys. Chem. C 114, 13118-13125 (2010). https://doi.org/10.1021/jp104488b

297. K. Zhang, M. Fujitsuka, Y. Du, T. Majima, 2D/2D heterostructured CdS/WS $\mathrm{W}_{2}$ with efficient charge separation improving $\mathrm{H}_{2}$ evolution under visible light irradiation. ACS Appl. Mater. Interfaces. 10, 20458-20466 (2018). https://doi. org/10.1021/acsami.8b04080

298. R.P. Schwarzenbach, T. Egli, T.B. Hofstetter, U.V. Gunten, B. Wehrli, Global water pollution and human health. Annu. Rev. Environ. Resour. 35, 10 (2010). https://doi.org/10.1146/ annurev-environ-100809-125342

299. Y. Li, K. Li, Y. Yang, L. Li, Y. Xing et al., Ultrathin g- $\mathrm{C}_{3} \mathrm{~N}_{4}$ nanosheets coupled with $\mathrm{AgIO}_{3}$ as highly efficient heterostructured photocatalysts for enhanced visible-light photocatalytic activity. Chemistry 21, 17739-17747 (2015). https://doi.org/10.1002/chem.201502945

300. J. Wang, L. Tang, G. Zeng, Y. Deng, Y. Liu et al., Atomic scale $\mathrm{g}-\mathrm{C}_{3} \mathrm{~N}_{4} / \mathrm{Bi}_{2} \mathrm{WO}_{6} 2 \mathrm{D} / 2 \mathrm{D}$ heterojunction with enhanced photocatalytic degradation of ibuprofen under visible light irradiation. Appl. Catal. B Environ. 209, 285-294 (2017). https://doi.org/10.1016/j.apcatb.2017.03.019

301. R. Bera, S. Kundu, A. Patra, 2D hybrid nanostructure of reduced graphene oxide-CdS nanosheet for enhanced photocatalysis. ACS Appl. Mater. Interfaces. 7, 13251 (2015). https://doi.org/10.1021/acsami.5b03800

302. Y. Liu, S. Xie, L. Hui, X. Wang, A highly efficient sunlight driven $\mathrm{ZnO}$ nanosheet photocatalyst: synergetic effect of p-doping and $\mathrm{MoS}_{2}$ atomic layer loading. ChemCatChem 6, 2522-2526 (2014). https://doi.org/10.1002/cctc.201402191

303. W.J. Ong, L.L. Tan, S.P. Chai, S.T. Yong, Graphene oxide as a structure-directing agent for the two-dimensional interface engineering of sandwich-like graphene-g- $\mathrm{C}_{3} \mathrm{~N}_{4}$ hybrid nanostructures with enhanced visible-light photoreduction of $\mathrm{CO}_{2}$ to methane. Chem. Commun. 51, 858-861 (2015). https://doi. org/10.1039/c4cc08996k

304. J. Sun, H. Zhang, L.H. Guo, L. Zhao, Two-dimensional interface engineering of a titania-graphene nanosheet composite for improved photocatalytic activity. ACS Appl. Mater. Interfaces. 5, 13035-13041 (2013). https://doi.org/10.1021/am403 $937 \mathrm{y}$

305. W. Ong, L.L. Tan, S.P. Chai, S.T. Yong, A. Mohamed, Surface charge modification via protonation of graphitic carbon nitride $\left(\mathrm{g}-\mathrm{C}_{3} \mathrm{~N}_{4}\right)$ for electrostatic self-assembly construction of $2 \mathrm{D} / 2 \mathrm{D}$ reduced graphene oxide $(\mathrm{rGO}) / \mathrm{g}-\mathrm{C}_{3} \mathrm{~N}_{4}$ nanostructures toward enhanced photocatalytic reduction of carbon dioxide to methane. Nano Energy 13, 757-770 (2015). https ://doi.org/10.1016/j.nanoen.2015.03.014

306. Y.T. Liang, B.K. Vijayan, O. Lyandres, K.A. Gray, M.C. Hersam, Effect of dimensionality on the photocatalytic behavior of carbon-titania nanosheet composites: charge transfer at nanomaterial interfaces. J. Phys. Chem. Lett. 3, 1760-1765 (2012). https://doi.org/10.1021/jz300491s

307. W. Tu, Y. Zhou, Q. Liu, Z. Tian, J. Gao et al., Robust hollow spheres consisting of alternating titania nanosheets and graphene nanosheets with high photocatalytic activity for $\mathrm{CO}_{2}$ conversion into renewable fuels. Adv. Funct. Mater. 22, 1215-1221 (2012). https://doi.org/10.1002/adfm.201102566

308. X. Chen, Y. Zhou, Q. Liu, Z. Li, J. Liu, Z. Zou, Ultrathin, single-crystal $\mathrm{WO}_{3}$ nanosheets by two-dimensional oriented attachment toward enhanced photocatalystic reduction of $\mathrm{CO}_{2}$ into hydrocarbon fuels under visible light. ACS Appl. Mater. Interfaces. 4, 3372-3377 (2012). https://doi.org/10.1021/ am300661s

309. W. Tu, Y. Li, L. Kuai, Y. Zhou, Q. Xu et al., Construction of unique two-dimensional $\mathrm{MoS}_{2}-\mathrm{TiO}_{2}$ hybrid nanojunctions: $\mathrm{MoS}_{2}$ as a promising cost-effective cocatalyst toward improved photocatalytic reduction of $\mathrm{CO}_{2}$ to methanol. Nanoscale 9, 9065-9070 (2017). https://doi.org/10.1039/ C7NR03238B 
310. L. Zhang, W. Wang, D. Jiang, E. Gao, S. Sun, Photoreduction of $\mathrm{CO}_{2}$ on $\mathrm{BioCl}$ nanoplates with the assistance of photoinduced oxygen vacancies. Nano Res. 8, 821-831 (2015). https ://doi.org/10.1007/s12274-014-0564-2

311. J.C. Wang, H.C. Yao, Z.Y. Fan, L. Zhang, J.S. Wang, S.Q. Zang, Z.J. Li, Indirect z-scheme BiOi/g- $\mathrm{C}_{3} \mathrm{~N}_{4}$ photocatalysts with enhanced photoreduction $\mathrm{CO}_{2}$ activity under visible light irradiation. ACS Appl. Mater. Interfaces. 8, 3765-3775 (2016). https://doi.org/10.1021/acsami.5b09901

312. S. Kawamura, M.C. Puscasu, Y. Yoshida, Y. Izumi, G. Carja, Tailoring assemblies of plasmonic silver/gold and zinc-gallium layered double hydroxides for photocatalytic conversion of carbon dioxide using UV-visible light. Appl. Catal. A Gen. 504, 238-247 (2015). https://doi.org/10.1016/j.apcat a.2014.12.042

313. H.C. Hsu, I. Shown, H.Y. Wei, Y.C. Chang, H.Y. Du et al., Graphene oxide as a promising photocatalyst for $\mathrm{CO}_{2}$ to methanol conversion. Nanoscale 5, 262-268 (2013). https:// doi.org/10.1039/C2NR31718D

314. B. Qiu, Q. Li, B. Shen, M. Xing, J. Zhang, Stöber-like method to synthesize ultradispersed $\mathrm{Fe}_{3} \mathrm{O}_{4}$ nanoparticles on graphene with excellent photo-fenton reaction and high-performance lithium storage. Appl. Catal. B Environ. 183, 216-223 (2016). https://doi.org/10.1016/j.apcatb.2015.10.053

315. L.L. Tan, W.J. Ong, S.P. Chai, B.T. Goh, A.R. Mohamed, Visible-light-active oxygen-rich $\mathrm{TiO}_{2}$ decorated 2D graphene oxide with enhanced photocatalytic activity toward carbon dioxide reduction. Appl. Catal. B Environ. 179, 160-170 (2015). https://doi.org/10.1016/j.apcatb.2015.05.024

316. A. Ali, W.C. Oh, A simple ultrasono-synthetic route of $\mathrm{PbSe}-$ graphene- $\mathrm{TiO}_{2}$ ternary composites to improve the photocatalytic reduction of $\mathrm{CO}_{2}$. Fuller. Nanotube Carbon Nanostruct. 25, 449-458 (2017). https://doi.org/10.1080/15363 83X.2017.1308354

317. L.L. Tan, W.J. Ong, S.P. Chai, A.R. Mohamed, Reduced graphene oxide- $\mathrm{TiO}_{2}$ nanocomposite as a promising visible-light-active photocatalyst for the conversion of carbon dioxide. Nanoscale Res. Lett. 8, 465 (2013). https://doi. org/10.1186/1556-276X-8-465

318. A. Wang, X. Li, Y. Zhao, W. Wu, J. Chen, H. Meng, Preparation and characterizations of $\mathrm{Cu}_{2} \mathrm{O}$ /reduced graphene oxide nanocomposites with high photo-catalytic performances. Powder Technol. 261, 42-48 (2014). https://doi. org/10.1016/j.powtec.2014.04.004

319. Y.F. Xu, M.Z. Yang, B.X. Chen, X.D. Wang, H.Y. Chen, D.B. Kuang, C.Y. Su, A CsPbBr 3 perovskite quantum dot/ graphene oxide composite for photocatalytic $\mathrm{co}_{2}$ reduction. J. Am. Chem. Soc. 139, 5660-5663 (2017). https:// doi.org/10.1021/jacs.7b00489

320. L. Kuai, Y. Zhou, W. Tu, P. Li, H. Li et al., Rational construction of a CdS/reduced graphene oxide/ $/ \mathrm{TiO}_{2}$ core-shell nanostructure as an all-solid-state $\mathrm{z}$-scheme system for $\mathrm{CO}_{2}$ photoreduction into solar fuels. RSC Adv. 5, 88409-88413 (2015). https://doi.org/10.1039/C5RA14374H

321. W. Dai, J. Yu, Y. Deng, X. Hu, T. Wang, X. Luo, Facile synthesis of $\mathrm{MoS}_{2} / \mathrm{Bi}_{2} \mathrm{WO}_{6}$ nanocomposites for enhanced
$\mathrm{CO}_{2}$ photoreduction activity under visible light irradiation. Appl. Surf. Sci. 403, 230-239 (2017). https://doi. org/10.1016/j.apsusc.2017.01.171

322. X. Jiao, X. Li, X. Jin, Y. Sun, J. Xu et al., Partially oxidized sns $_{2}$ atomic layers achieving efficient visible-light-driven $\mathrm{CO}_{2}$ reduction. J. Am. Chem. Soc. 139, 18044-18051 (2017). https://doi.org/10.1021/jacs.7b10287

323. Q. Xin, H. Shah, A. Nawaz, W. Xie, M.Z. Akram et al., Antibacterial carbon-based nanomaterials. Adv. Mater. 31, 1804838 (2019). https://doi.org/10.1002/adma.201804838

324. J. Ge, Y. Zhang, S.J. Park, Recent advances in carbonaceous photocatalysts with enhanced photocatalytic performances: a mini review. Materials 12, 1916 (2019). https:// doi.org/10.3390/ma12121916

325. R. Wang, X. Zhang, F. Li, D. Cao, M. Pu et al., Energylevel dependent $\mathrm{H}_{2} \mathrm{O}_{2}$ production on metal-free, carboncontent tunable carbon nitride photocatalysts. J. Energy Chem. 27, 343-350 (2018). https://doi.org/10.1016/j.jeche m.2017.12.014

326. D. Tasis, N. Tagmatarchis, A. Bianco, M. Prato, Chemistry of carbon nanotubes. Chem. Rev. 106, 1105-1136 (2006). https://doi.org/10.1021/cr050569o

327. S. Zhao, T. Guo, X. Li, T. Xu, B. Yang, X. Zhao, Carbon nanotubes covalent combined with graphitic carbon nitride for photocatalytic hydrogen peroxide production under visible light. Appl. Catal. B Environ. 224, 725-732 (2018). https://doi.org/10.1016/j.apcatb.2017.11.005

328. D.L. Long, E. Burkholder, L. Cronin, Polyoxometalate clusters, nanostructures and materials: from self assembly to designer materials and devices. Chem. Soc. Rev. 36, 105-121 (2007). https://doi.org/10.1039/B502666K

329. X.B. Han, Z.M. Zhang, T. Zhang, Y.G. Li, W. Lin et al., Polyoxometalate-based cobalt-phosphate molecular catalysts for visible light-driven water oxidation. J. Am. Chem. Soc. 136, 5359-5366 (2014). https://doi.org/10.1021/ja412 $886 \mathrm{e}$

330. X.J. Kong, Z. Lin, Z.M. Zhang, T. Zhang, W. Lin, Hierarchical integration of photosensitizing metal-organic frameworks and nickel-containing polyoxometalates for efficient visiblelight-driven hydrogen evolution. Angew. Chem. Int. Ed. 55, 6411-6416 (2016). https://doi.org/10.1002/anie.201600431

331. J. Zhou, W. Chen, C. Sun, L. Han, C. Qin et al., Oxidative polyoxometalates modified graphitic carbon nitride for visible-light $\mathrm{CO}_{2}$ reduction. ACS Appl. Mater. Interfaces. 9, 11689-11695 (2017). https://doi.org/10.1021/acsami.7b017 21

332. S. Zhao, X. Zhao, H. Zhang, J. Li, Y. Zhu, Covalent combination of polyoxometalate and graphitic carbon nitride for lightdriven hydrogen peroxide production. Nano Energy 35, 405414 (2017). https://doi.org/10.1016/j.nanoen.2017.04.017

333. S. Zhao, X. Zhao, S. Ouyang, Y. Zhu, Polyoxometalates covalently combined with graphitic carbon nitride for photocatalytic hydrogen peroxide production. Catal. Sci. Technol. 8, 1686-1695 (2018). https://doi.org/10.1039/C8CY00043C

334. S. Zhao, X. Zhao, Polyoxometalates-derived metal oxides incorporated into graphitic carbon nitride framework for 
photocatalytic hydrogen peroxide production under visible light. J. Catal. 366, 98-106 (2018). https://doi.org/10.1016/j. jcat.2018.08.003

335. S. Zhao, X. Zhao, Insights into the role of singlet oxygen in the photocatalytic hydrogen peroxide production over polyoxometalates-derived metal oxides incorporated into graphitic carbon nitride framework. Appl. Catal. B Environ. 250, 408418 (2019). https://doi.org/10.1016/j.apcatb.2019.02.031

336. X. Jiang, P. Wang, J. Zhao, 2d covalent triazine framework: a new class of organic photocatalyst for water splitting. J. Mater. Chem. A 3, 7750-7758 (2015). https://doi. org/10.1039/C4TA03438D

337. Y. Shiraishi, S. Kanazawa, Y. Kofuji, H. Sakamoto, S. Ichikawa, S. Tanaka, T. Hirai, Sunlight-driven hydrogen peroxide production from water and molecular oxygen by metal-free photocatalysts. Angew. Chem. Int. Ed. 53, 13454-13459 (2014). https://doi.org/10.1002/anie.201407938

338. Y. Kofuji, S. Ohkita, Y. Shiraishi, H. Sakamoto, S. Tanaka, S. Ichikawa, T. Hirai, Graphitic carbon nitride doped with biphenyl diimide: efficient photocatalyst for hydrogen peroxide production from water and molecular oxygen by sunlight. ACS Catal. 6, 7021-7029 (2016). https://doi.org/10.1021/ acscatal.6b02367

339. Y. Kofuji, S. Ohkita, Y. Shiraishi, H. Sakamoto, S. Ichikawa, S. Tanaka, T. Hirai, Mellitic triimide-doped carbon nitride as sunlight-driven photocatalysts for hydrogen peroxide production. Catal. Sci. Technol. 5, 6478-6485 (2017). https://doi. org/10.1021/acssuschemeng.7b00575

340. Y. Kofuji, Y. Isobe, Y. Shiraishi, H. Sakamoto, S. Tanaka, S. Ichikawa, T. Hirai, Carbon nitride-aromatic diimide-graphene nanohybrids: metal-free photocatalysts for solar-tohydrogen peroxide energy conversion with $0.2 \%$ efficiency. J. Am. Chem. Soc. 138, 10019-10025 (2016). https://doi. org/10.1021/jacs.6b05806

341. Y. Kofuji, Y. Isobe, Y. Shiraishi, H. Sakamoto, S. Ichikawa, S. Tanaka, T. Hirai, Hydrogen peroxide production on a carbon nitride-boron nitride-reduced graphene oxide hybrid photocatalyst under visible light. ChemCatChem 10, 2070-2077 (2018). https://doi.org/10.1002/cctc.201701683

342. L. Yang, G. Dong, D.L. Jacobs, Y. Wang, L. Zang, C. Wang, Two-channel photocatalytic production of $\mathrm{H}_{2} \mathrm{O}_{2}$ over $\mathrm{g}-\mathrm{C}_{3} \mathrm{~N}_{4}$ nanosheets modified with perylene imides. J. Catal. 352, 274-281 (2017). https://doi.org/10.1016/j. jcat.2017.05.010

343. H.I. Kim, Y. Choi, S. Hu, W. Choi, J.H. Kim, Photocatalytic hydrogen peroxide production by anthraquinone-augmented polymeric carbon nitride. Appl. Catal. B Environ. 229, 121129 (2018). https://doi.org/10.1016/j.apcatb.2018.01.060

344. F. Xue, Y. Si, M. Wang, M. Liu, L. Guo, Toward efficient photocatalytic pure water splitting for simultaneous $\mathrm{H}_{2}$ and $\mathrm{H}_{2} \mathrm{O}_{2}$ production. Nano Energy 62, 823-831 (2019). https:// doi.org/10.1016/j.nanoen.2019.05.086

345. Y. Shiraishi, S. Kanazawa, Y. Sugano, D. Tsukamoto, H. Sakamoto, S. Ichikawa, T. Hirai, Highly selective production of hydrogen peroxide on graphitic carbon nitride $\left(\mathrm{g}-\mathrm{C}_{3} \mathrm{~N}_{4}\right)$ photocatalyst activated by visible light. ACS Catal. 4, 774780 (2014). https://doi.org/10.1021/cs401208c

346. B.O. Burek, D.W. Bahnemann, J.Z. Bloh, Modeling and optimization of the photocatalytic reduction of molecular oxygen to hydrogen peroxide over titanium dioxide. ACS Catal. 9, 25-37 (2018). https://doi.org/10.1021/acscatal.8b03638

347. R. Cai, Y. Kubota, A. Fujishima, Effect of copper ions on the formation of hydrogen peroxide from photocatalytic titanium dioxide particles. J. Catal. 219, 214-218 (2003). https://doi. org/10.1016/S0021-9517(03)00197-0

348. S. Kim, G.H. Moon, G. Kim, U. Kang, H. Park, W. Choi, $\mathrm{TiO}_{2}$ complexed with dopamine-derived polymers and the visible light photocatalytic activities for water pollutants. J. Catal. 346, 92-100 (2017). https://doi.org/10.1016/j. jcat.2016.11.027

349. X. Zeng, Z. Wang, G. Wang, T.R. Gengenbach, D.T. McCarthy et al., Highly dispersed $\mathrm{TiO}_{2}$ nanocrystals and $\mathrm{wo}_{3}$ nanorods on reduced graphene oxide: Z-scheme photocatalysis system for accelerated photocatalytic water disinfection. Appl. Catal. B Environ. 218, 163-173 (2017). https://doi. org/10.1016/j.apcatb.2017.06.055

350. X. Xiong, X. Zhang, S. Liu, J. Zhao, Y. Xu, Sustained production of $\mathrm{H}_{2} \mathrm{O}_{2}$ in alkaline water solution using borate and phosphate-modified $\mathrm{Au} / \mathrm{TiO}_{2}$ photocatalysts. Photochem. Photobiol. Sci. 17, 1018-1022 (2018). https://doi.org/10.1039/ C8PP00177D

351. G. Zuo, B. Li, Z. Guo, L. Wang, F. Yang et al., Efficient photocatalytic hydrogen peroxide production over $\mathrm{TiO}_{2}$ passivated by $\mathrm{SnO}_{2}$. Catalysts 9, 623 (2019). https://doi. org/10.3390/catal9070623

352. Y. Shiraishi, Y. Kofuji, H. Sakamoto, S. Tanaka, S. Ichikawa, T. Hirai, Effects of surface defects on photocatalytic $\mathrm{H}_{2} \mathrm{O}_{2}$ production by mesoporous graphitic carbon nitride under visible light irradiation. ACS Catal. 5, 3058-3066 (2015). https ://doi.org/10.1021/acscatal.5b00408

353. S. Kim, G.H. Moon, H. Kim, Y. Mun, P. Zhang, J. Lee, W. Choi, Selective charge transfer to dioxygen on KPF6-modified carbon nitride for photocatalytic synthesis of $\mathrm{H}_{2} \mathrm{O}_{2}$ under visible light. J. Catal. 357, 51-58 (2018). https://doi. org/10.1016/j.jcat.2017.10.002

354. L. Shi, L. Yang, W. Zhou, Y. Liu, L. Yin et al., Photoassisted construction of holey defective $\mathrm{g}-\mathrm{C}_{3} \mathrm{~N}_{4}$ photocatalysts for efficient visible-light-driven $\mathrm{H}_{2} \mathrm{O}_{2}$ production. Small 14, 1703142 (2018). https://doi.org/10.1002/smll.201703142

355. Z. Wei, M. Liu, Z. Zhang, W. Yao, H. Tan, Y. Zhu, Efficient visible-light-driven selective oxygen reduction to hydrogen peroxide by oxygen-enriched graphitic carbon nitride polymers. Energy Environ. Sci. 11, 2581-2589 (2018). https:// doi.org/10.1039/C8EE01316K

356. Y. Kusuyama, S. Tanaka, K. Sakatsuji, T. Nishihara, K. Saito et al., Central pontine myelinolysis. Pathol. Int. 32, 725-732 (2008). https://doi.org/10.1111/j.1440-1827.1982.tb02074.x

357. S. Zhao, X. Wang, M. Huo, Catalytic wet air oxidation of phenol with air and micellar molybdovanadophosphoric polyoxometalates under room condition. Appl. Catal. B 
Environ. 97, 127-134 (2010). https://doi.org/10.1016/j.apcat b.2010.03.032

358. S. Kato, J. Jung, T. Suenobu, S. Fukuzumi, Production of hydrogen peroxide as a sustainable solar fuel from water and dioxygen. Energy Environ. Sci. 6, 3756-3764 (2013). https ://doi.org/10.1039/C3EE42815J

359. H. Hirakawa, S. Shiota, Y. Shiraishi, H. Sakamoto, S. Ichikawa, T. Hirai, Au nanoparticles supported on $\mathrm{BiVO}_{4}$ : effective inorganic photocatalysts for $\mathrm{H}_{2} \mathrm{O}_{2}$ production from water and $\mathrm{O}_{2}$ under visible light. ACS Catal. 6, 4976-4982 (2016). https://doi.org/10.1021/acscatal.6b01187
360. X. Jie, Z. Chen, H. Zhang, G. Lin, H. Lin, X. Wang, J. Long, $\mathrm{Cd}_{3}\left(\mathrm{C}_{3} \mathrm{~N}_{3} \mathrm{~S}_{3}\right)_{2}$ coordination polymer/graphene nanoarchitectures for enhanced photocatalytic $\mathrm{H}_{2} \mathrm{O}_{2}$ production under visible light. Sci. Bull. 62, 610-618 (2017). https://doi. org/10.1016/j.scib.2017.04.013

361. T. Inagami, N. Yokosawa, N. Takahashi, Y. Takii, Partial purification of prorenin and activation by kallikreins: a possible new link between renin and kallikrein systems. Adv. Exp. Med. Biol. 120, 415-428 (1979) 